\title{
The Role of Bovine Cathelicidins in Host Defence
}

By

Blake William Paget

\begin{abstract}
A thesis
submitted to the Victoria University of Wellington in fulfilment of the requirements for the degree of

Doctor of Philosophy
\end{abstract}

Victoria University of Wellington

2014 


\section{Abstract}

Cattle are constantly exposed to environmental pathogens and are susceptible to a number of diseases which cause significant economic loss or animal welfare concerns. The mucosal surface is a key barrier to infection and a greater understanding of bovine mucosal immunology may lead to improved disease management strategies. The cathelicidins are a family of host defence proteins which may play an important role in this defensive barrier.

The cathelicidins are composed of a globular cathelin-like domain (CLD) and a C-terminal antimicrobial peptide (AMP) domain. The CLD is highly conserved across all species in which cathelicidins are found, yet the role of the CLD during infection has not been firmly established. The first aim of this thesis was to produce functional recombinant bovine CLD as a precursor to further experiments. However, the recombinant protein bound less lipopolysaccharide, was unable to agglutinate microbes, and was unable to permeabilise neutrophil membranes when compared to the activity of a native CLD preparation. Although further studies were not carried out with recombinant CLD, these results demonstrated that cell death induced by the native CLD and the agglutination of microbes potentially contribute to a broad anti-inflammatory role for the CLD during infection.

In contrast to humans and mice where only one cathelicidin isoform is expressed, bovine express seven cathelicidins, with variable AMP domains. Therefore the second aim of this thesis was to profile the effect of bovine cathelicidin AMPs on neutrophil function. The bovine AMPs were able to modify a number of activities. Migration and reactive oxygen species (ROS) production were enhanced by several peptides while ROS production was inhibited by others. When investigated in further detail, linear Bac1 (bactenecin), Bac5 and BMAP-34 (bovine myeloid antimicrobial peptide) were able to dose-dependently induce or inhibit several key neutrophil functions including migration, degranulation, respiratory burst and phagocytosis, indicating significant roles in differential modulation of immune responses. In particular, Bac5 was able to differentially modify neutrophil respiratory burst without significant disruption to cellular homeostasis, which suggested Bac5 was acting via an intracellular mechanism. 
The third aim of this thesis was to investigate the mechanism by which Bac5 modulated neutrophil function. The results demonstrated the ability of Bac5 to be internalised by neutrophils and that Bac5 inhibition of $\mathrm{p} 47^{\text {phox }}$ binding to nicotinamide adenine dinucleotide phosphate (NADPH) oxidase is a possible explanation for the differential induction and inhibition of extracellular ROS production. $\beta$-actin was identified as a major intracellular target for Bac5 and is consistent with the ability of Bac5 to modulate multiple neutrophil functions.

In summary, this thesis demonstrates that the bovine cathelicidins have multiple roles in host defence. The conserved CLD appears to have an anti-inflammatory role through an as yet undefined mechanism. The variable AMPs together have multifaceted roles which may act in concert to promote elimination of pathogens and regulate excessive detrimental neutrophil activity. The combined effect of these roles will facilitate clearance of pathogens during infection and aid in the resolution of the innate inflammatory response at mucosal surfaces. 


\section{Acknowledgements}

I wish to express my extreme gratitude to my supervisors Dr Brendan Haigh and Dr Jacquie Harper for their patience, guidance, encouragement, optimism and enthusiasm during the course of my studies. Bringing this thesis to fruition would not have been possible without their valuable contributions.

Thank you to Dr Julie Cakebread and Marita Broadhurst for proof-reading sections of this thesis, much appreciated. Special thanks to Robert Wieliczko, Marita, Grant Smolenski and Dr Tom Wheeler for all their technical advice, and always going out of their way to assist with reagents, equipment, protocols or analysis. For conducting the mass spectrometry analyses and providing associated technical advice, thank you to Grant, Irina Boggs, Ancy Thomas and Dr Stefan Clerens, and to Megan Callaghan for conducting the microbiology work. Thank you to Dr Paul Harris and Rachel Brunt for assistance with protein purification, and to Robin McDonald for flow cytometry training and advice. Thanks to everybody else in the Dairy Science Building and the wider Ruakura Research Campus for making Ruakura a fun and interesting place to work, play and socialise.

Thanks to Dr Willie-John Martin, Rene McLaughlin, Lisa Shaw, Odette Shaw and Stefanie Steiger of the Arthritis and Inflammation Group at the Malaghan Institute of Medical Research. Their help and support meant a lot to me and I sincerely wish them all the best in their scientific endeavours. Thank you to Kylie Price for flow cytometry training and advice and also to Lindsay and Travis Ancelet for their hospitality during my residencies in Wellington. I am grateful to Dr Barry O'Brien of the University of Waikato for confocal microscopy training and assistance and to the Ruakura and Taylor Preston abattoir staff for assisting in blood collections. The financial assistance for this thesis from the Ministry of Business, Innovation and Employment (grant C10x0707) was also very much appreciated.

Last but not least, many thanks to my family and friends for their encouragement and support, and to those who have put up with me over these years, Alastair, Laura, Tim, Lydia, Abbie and Jenna - cheers. 


\section{Table of Contents}

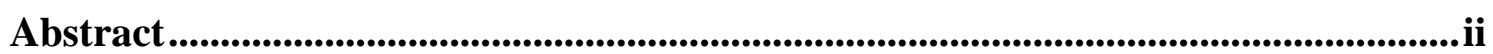

Acknowledgements.................................................................................................................... iv

Table of Contents ........................................................................................................... v

List of Figures..................................................................................................................................xiii

List of Tables ...............................................................................................................................

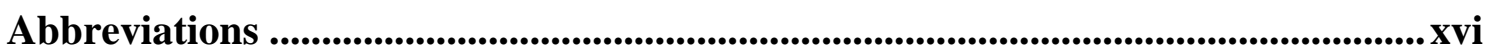

Chapter 1: General Introduction........................................................................................1

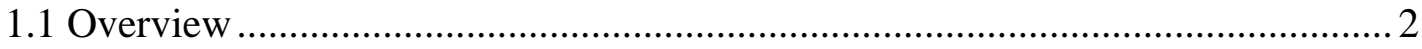

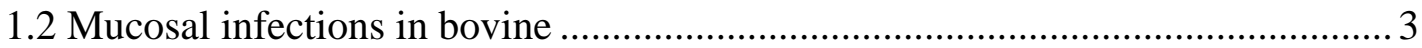

1.3 Host defence in bovine ............................................................................

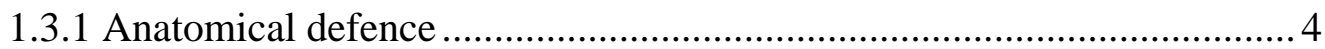

1.3.2 Innate cellular defence ....................................................................

1.3.2.2 Adhesion and diapedesis......................................................... 6

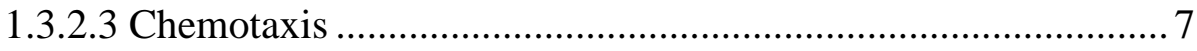

1.3.2.4 Degranulation .................................................................... 8

1.3.2.5 Respiratory burst............................................................. 8

1.3.2.6 Phagocytosis ........................................................................... 10

1.3.2.7 Cellular functions in the mammary gland and milk ................. 10

1.3.3 Innate humoral defence ……………………………………………..... 11

1.3.3.1 Complement system.............................................................. 11

1.3.3.2 Epithelial cell secretions and milk ........................................... 12

1.3.3.3 Neutrophil secreted proteins and peptides ............................... 13

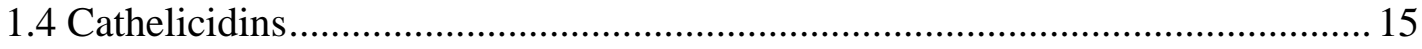

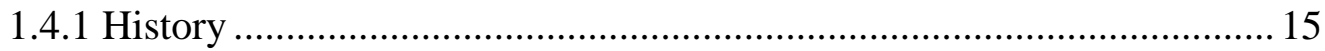

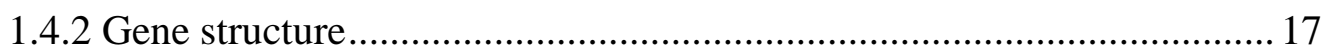

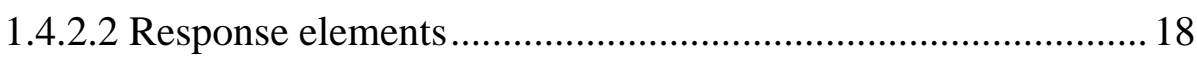

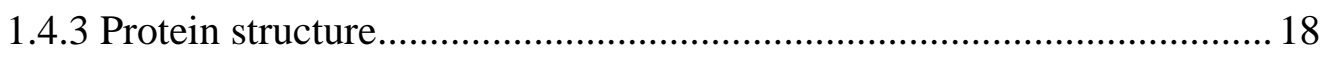

1.4.4 Localisation, processing and release …………………….................... 20 


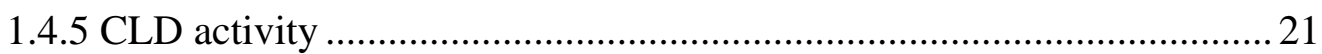

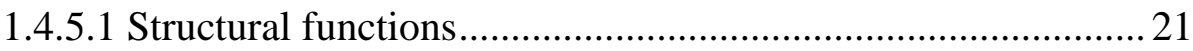

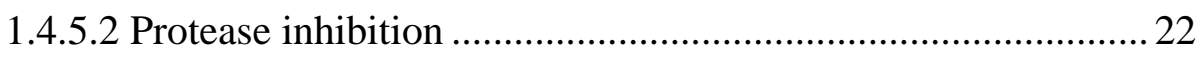

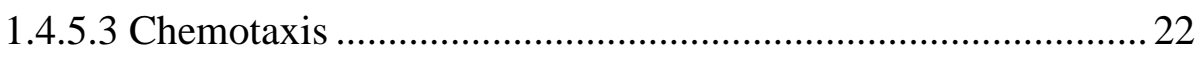

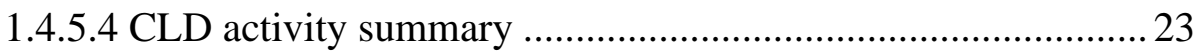

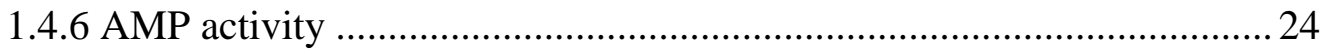

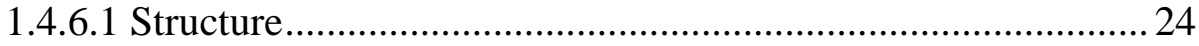

1.4.6.1 Antimicrobial activity ........................................................... 24

1.4.6.2 Clinical applications of AMPs..................................................2 26

1.4.6.3 Immunomodulatory activity ……………………………....... 27

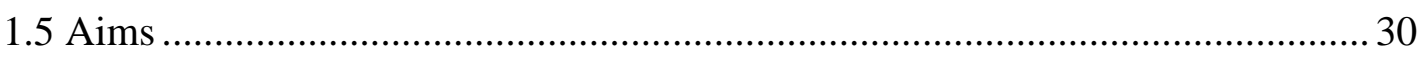

Chapter 2: Materials and Methods ......................................................................................31

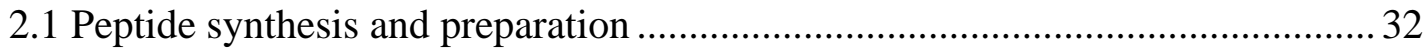

2.2 In-house antibody preparation........................................................................... 34

2.2.1 Cathelicidin-specific A14 and A32 antibodies.........................................34

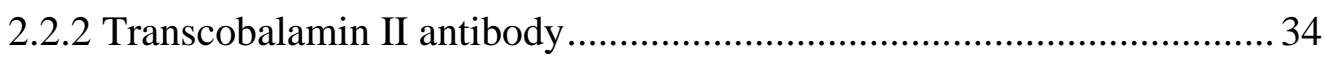

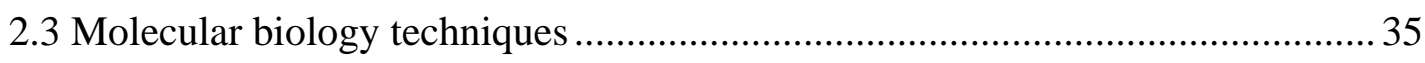

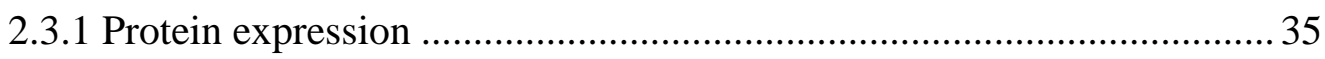

2.3.1.1 Open reading frame selection, PCR and cloning ......................35

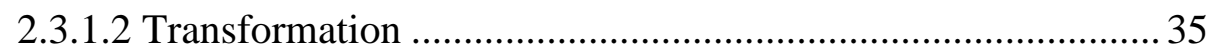

2.3.1.3 Small scale expression test ........................................................ 35

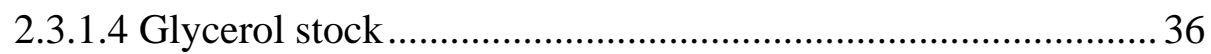

2.3.1.5 Large scale expression of rbCLD ............................................ 36

2.3.1.6 Large scale expression of rbCath 1 .............................................. 36

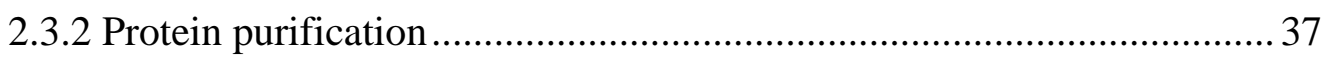

2.3.2.1 Column preparation and storage ................................................ 37

2.3.2.2 IMAC purification of rbCLD.................................................... 37

2.3.2.3 IMAC purification and on-column refolding of

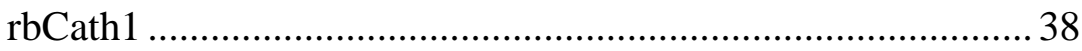

2.3.2.4 Concentration of purified protein ............................................. 38

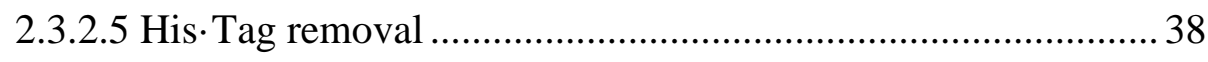

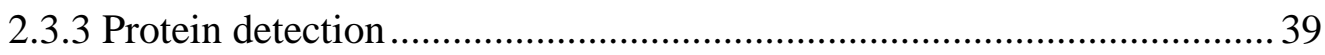




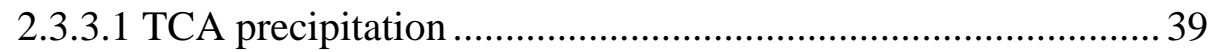

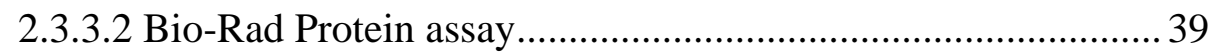

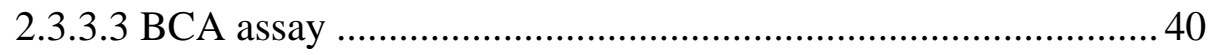

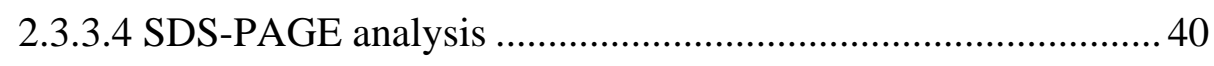

2.3.3.5 Western blot procedure ...................................................... 41

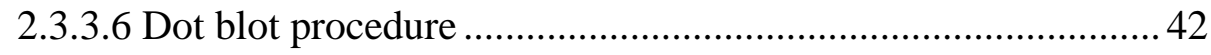

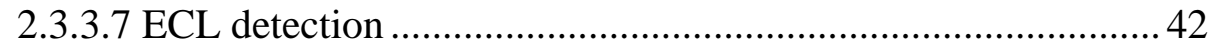

2.3.3.8 Far-western blot procedure ................................................ 43

2.3.3.9 Semi-quantitative analysis of polyacrylamide gels and

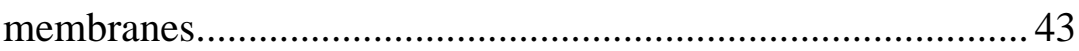

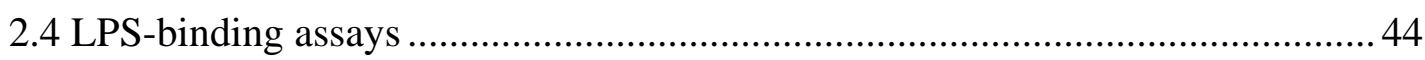

2.4.1 Recombinant cathelicidin LPS-binding assay ................................... 44

2.4.2 Cathelicidin AMP LPS-binding assay ............................................... 44

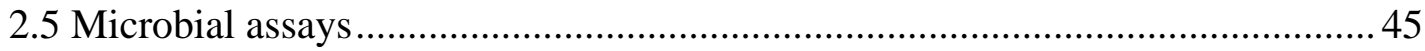

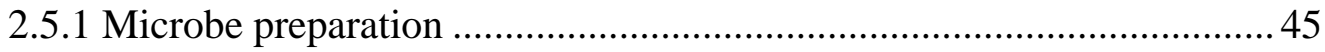

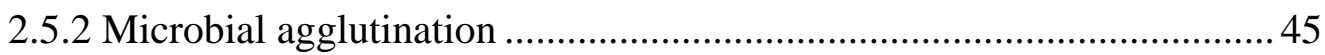

2.5.3 Minimal inhibitory concentration determination............................... 45

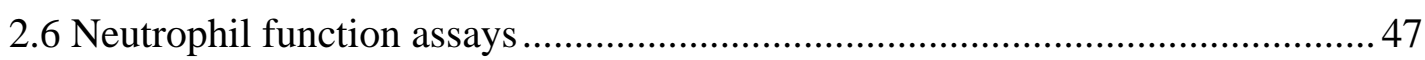

2.6.1 Preparation of neutrophils from bovine blood.................................... 47

2.6.2 Detection of apoptotic and necrotic cells ........................................... 48

2.6.3 Detection of membrane permeabilisation.......................................... 49

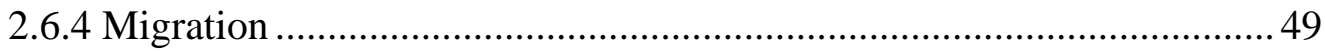

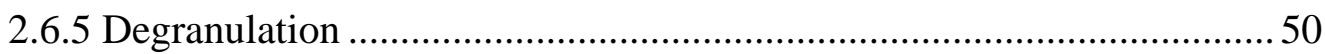

2.6.5.1 Measurement of primary granule release ...............................51

2.6.5.2 Measurement of secondary granule release ........................... 51

2.6.5.3 Measurement of large granule release ................................... 51

2.6.5.4 Measurement of secretory vesicle release ..............................52

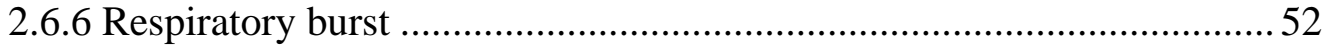

2.6.6.1 Measurement of extracellular ROS production .......................52

2.6.6.2 Measurement of intracellular ROS production........................53

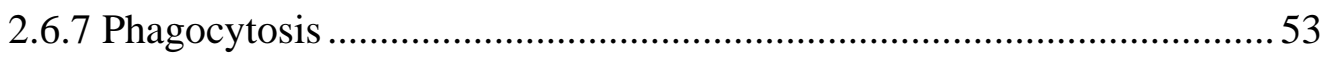

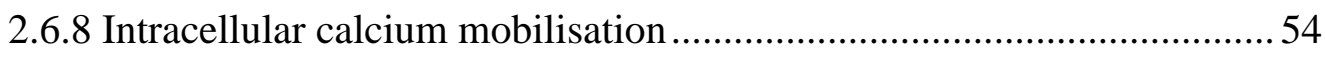

2.6.8.1 Preparation of zymosan-activated serum ...............................55 


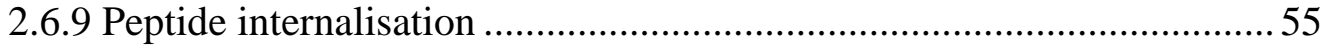

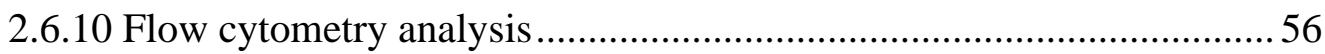

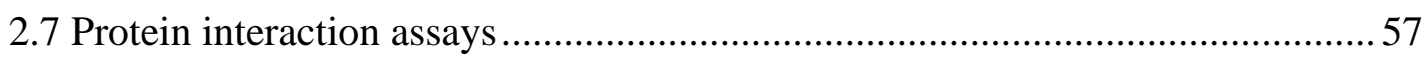

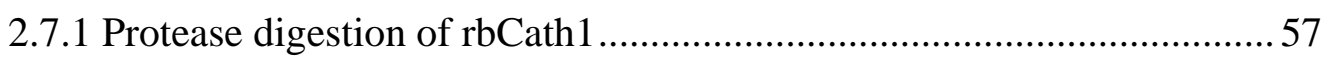

2.7.2 Ex vivo production of cathelicidin-1 peptides .......................................57

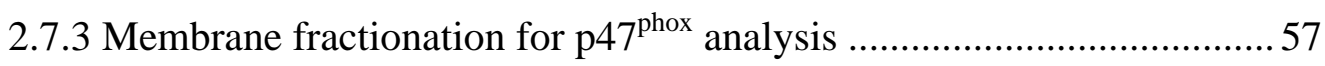

2.7.4 Preparation of whole neutrophil lysate ...............................................58

2.7.5 Analysis of Bac5 protein interactions.................................................59

2.7.6 Immunopurification of cellular $\beta$-actin ................................................59

2.7.7 Co-immunopurification of $\beta$-actin and Bac5-FITC............................. 60

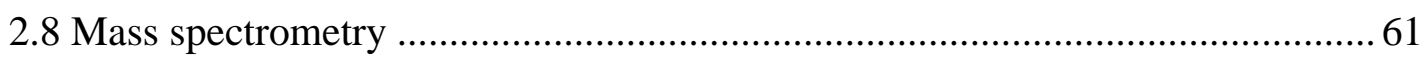

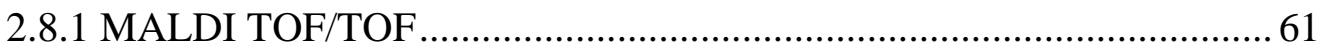

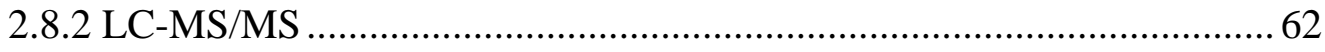

2.8.2.1 Preparation of Bac5 protein interaction samples .................... 62

2.8.2.2 Preparation of ex vivo produced cathelicidin-1

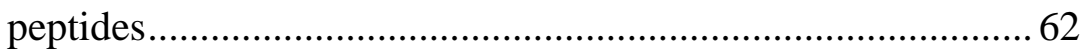

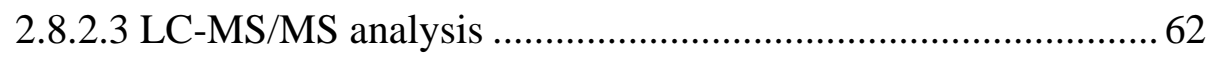

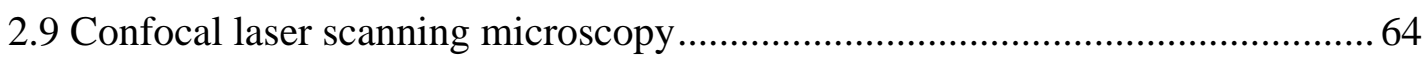

2.9.1 Slide preparation for phagocytosis analysis ...................................... 64

2.9.2 Slide preparation for colocalisation analysis ........................................ 64

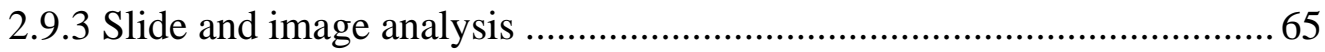

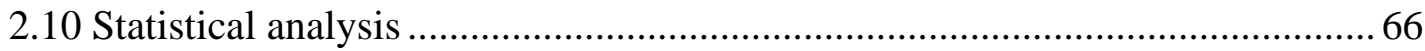

Chapter 3: Expression and Activity of Recombinant Cathelicidin-1......................... 67

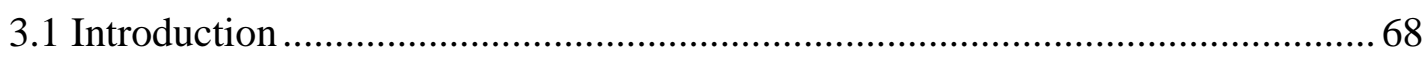

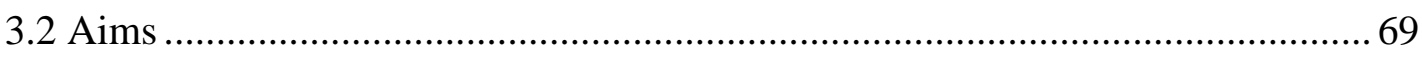

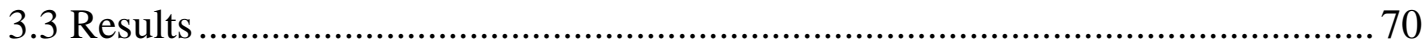

3.3.1 Expression and purification of rbCLD and rbCath1 ........................... 70

3.3.1.1 Bacterial expression constructs............................................ 70

3.3.1.2 Small scale expression test for rbCLD and rbCath1 .............. 72

3.3.1.3 Large scale expression and purification of rbCLD ................. 72

3.3.1.4 Large scale expression and purification of rbCath1 ............... 73

3.3.1.5 Mass spectrometry analysis of rbCLD …............................. 75 


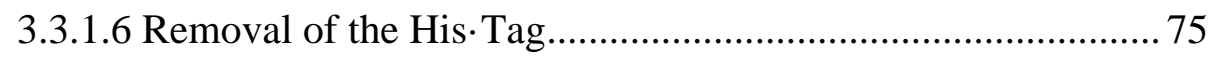

3.3.2 Activity and cytotoxicity of rbCLD and rbCath 1 ................................ 77

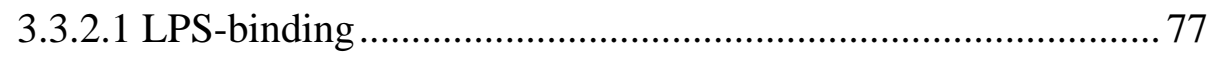

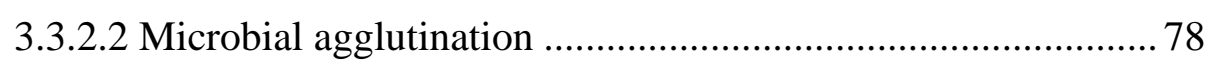

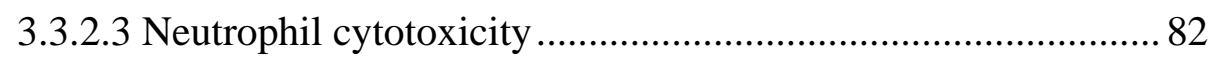

3.3.3 Investigation of a second AMP produced by cathelicidin-1 ................. 83

3.3.3.1 Digestion of rbCath1 with neutrophil elastase ........................ 83

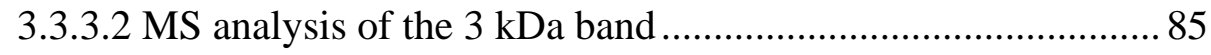

3.3.3.3 MS analysis of stimulated neutrophil culture media ............... 85

3.3.4 Investigation of RITK peptide activity .............................................. 88

3.3.4.1 MIC determination.............................................................. 88

3.3.4.2 Neutrophil respiratory burst.............................................. 88

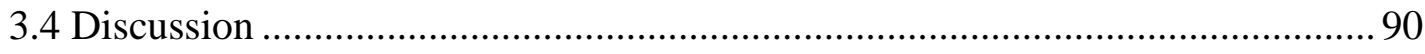

3.4.1 Expression and activity of rbCLD and rbCath1 ..................................90

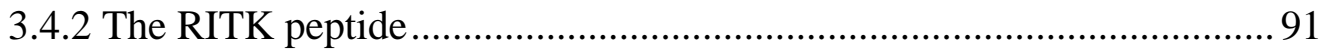

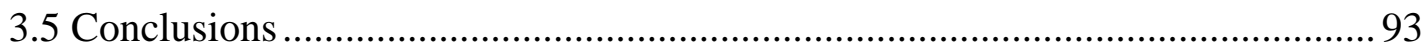

Chapter 4: Modulation of Bovine Neutrophil Function by Cathelicidin AMPs ..... 94

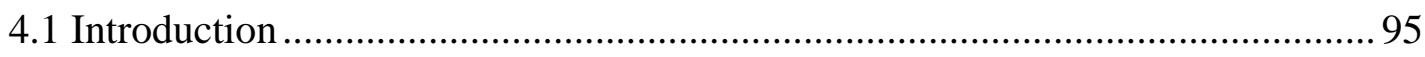

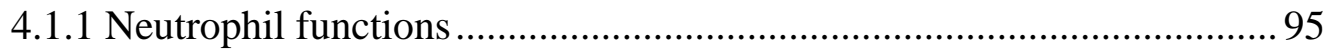

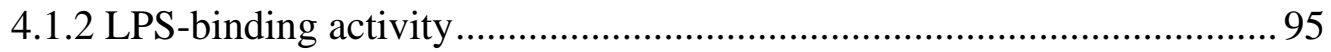

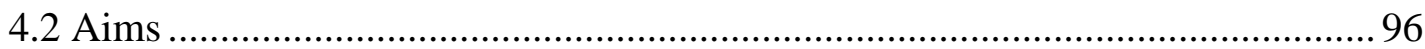

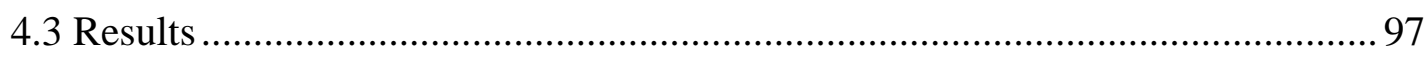

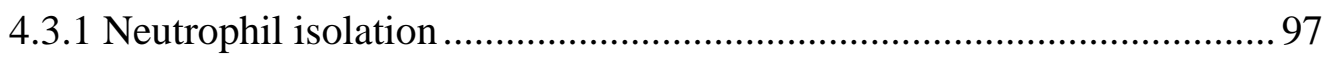

4.3.2 Primary screen of neutrophil function ................................................ 97

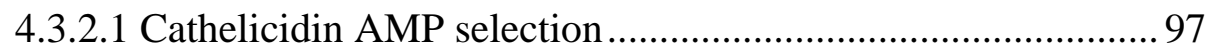

4.3.2.2 Bovine $\alpha$-helical peptides induce cytotoxicity......................... 98

4.3.2.3 BMAP-28 induces membrane permeabilisation ..................... 98

4.3.2.4 Multiple bovine AMPs induce neutrophil migration............... 99

4.3.2.5 Degranulation is only affected by BMAP-28 treatment ........ 101

4.3.2.6 Bovine AMPs have different effects on respiratory

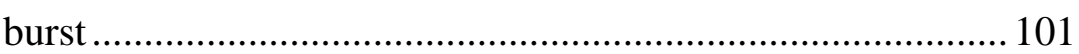

4.3.2.7 Bac5 enhances phagocytosis.............................................. 101 
4.3.2.8 Intracellular calcium mobilisation is not affected by

AMP treatment

4.3.2.9 Summary of the primary screen analysis of neutrophil function

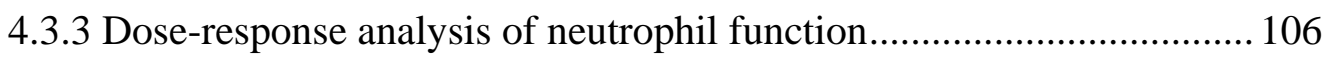

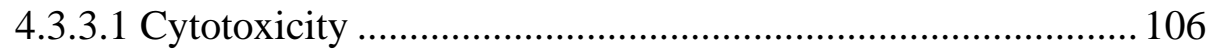

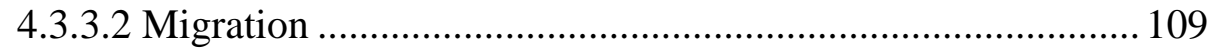

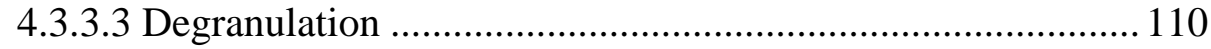

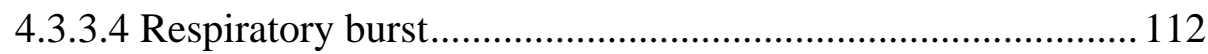

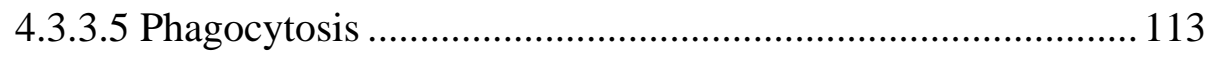

4.3.3.6 Intracellular calcium mobilisation ........................................ 113

4.3.3.7 Summary of the dose-response analysis of neutrophil

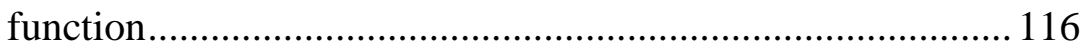

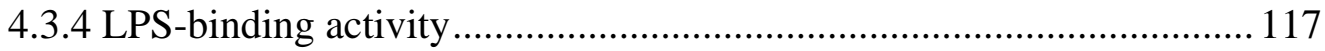

4.3.5 AMP-induced cytokine release from bovine neutrophils ................... 117

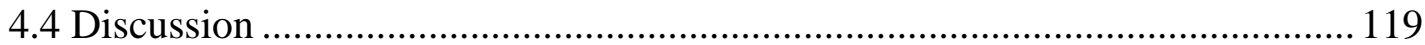

4.4.1 The effects of bovine cathelicidin AMPs on neutrophil function ........ 119

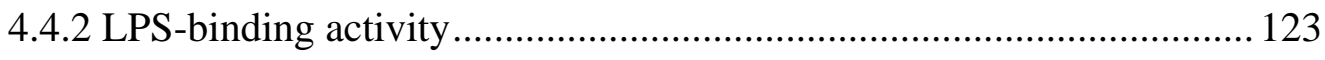

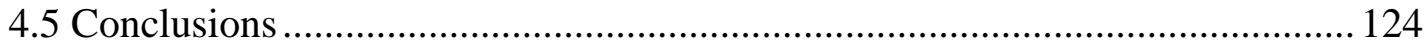

Chapter 5: The Mechanism of Bac5 Regulation of the Respiratory Burst............. 125

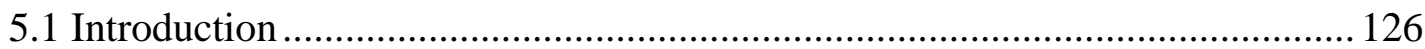

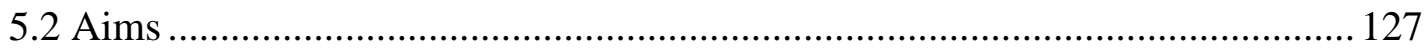

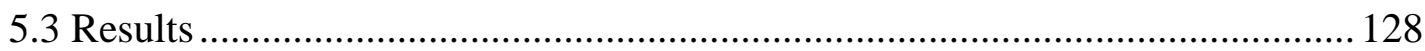

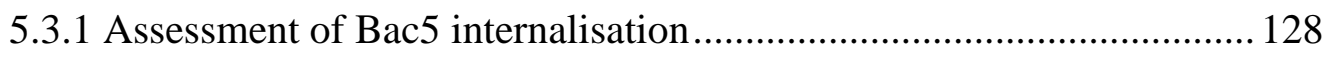

5.3.2 Bac5 induces intracellular ROS production ...................................... 130

5.3.3 Bac5 colocalises with intracellular ROS ......................................... 132

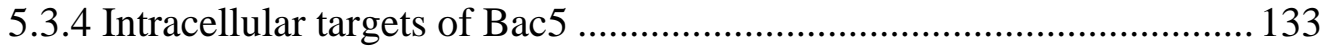

5.3.4.1 Bac5-induced ROS production does not involve

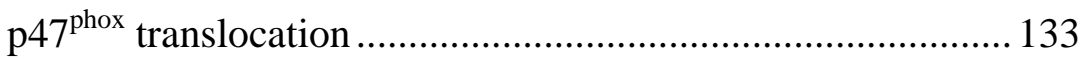

5.3.4.2 Bac5 binds to several intracellular neutrophil proteins ......... 135

5.3.4.3 $\beta$-actin is an intracellular target of Bac5 ............................. 135

5.3.5 Confirmation of the Bac5- $\beta$-actin interaction..................................... 138 
5.3.5.1 Bac5 binds immunopurified cellular $\beta$-actin 138

5.3.5.2 Bac5 does not readily co-purify with cellular $\beta$-actin 140

5.3.5.3 Co-immunopurification of Bac5 and purified $\beta$-actin 142

5.3.6 Bac5 colocalises with cytoplasmic $\beta$-actin 144

5.3.7 Bac5, intracellular ROS and $\beta$-actin have a cytoplasmic association 145

5.3.8 Activated neutrophils 146

5.3.8.1 Intracellular ROS is not inhibited by Bac5 146

5.3.8.2 Bac5 prevents $\mathrm{p} 47^{\text {phox }}$ translocation in activated neutrophils

5.3.8.3 Colocalisation of Bac5, intracellular ROS and $\beta$-actin is unchanged in activated cells

5.4 Discussion 150

5.5 Conclusion 154

Chapter 6: General Discussion 155

6.1 Overview 156

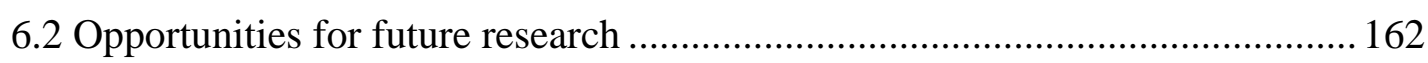

6.2.1 The origin of the immunomodulatory activity of the CLD ................. 162

6.2.2 Toward identifying in vivo production of the RITK peptide. 163

6.2.3 Establishing the mechanisms of linear Bac1 and BMAP-34 activity

6.2.4 Establishing the follow-on mechanisms of Bac5-actin interactions

References 166

Appendix 1: Bovine AMP Interactions with Cytokine Antibodies. 194

1 Interaction with ELISA antibodies. 194

2 Significance of the AMP-antibody interactions. 197

Appendix 2: Solutions and Reagents 198

1 General solutions 198 


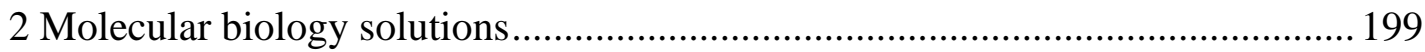

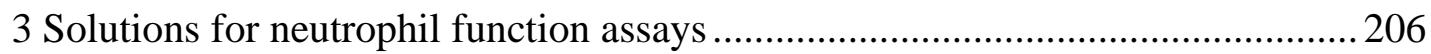

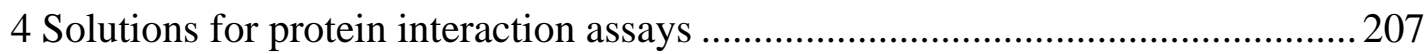

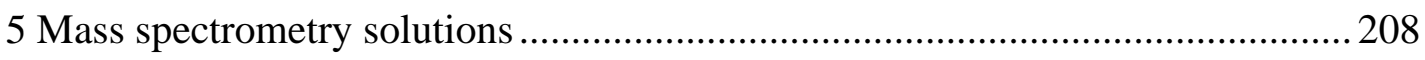




\section{List of Figures}

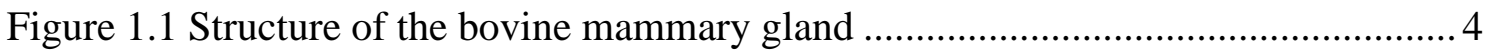

Figure 1.2 Myeloid-derived innate immune cells and associated receptors ....................5

Figure 1.3 The NADPH oxidase complex in resting and activated cells....................... 9

Figure 1.4 Domain structure and multiple sequence alignment of cathelicidins ............ 16

Figure 1.5 Exon and intron structure of cathelicidin genes ...................................... 17

Figure 1.6 Topology and three-dimensional structure of the human CLD .................... 19

Figure 3.1 Protein coding regions of the constructs expressed in E. coli ...................... 71

Figure 3.2 SDS-PAGE analysis of the expression and purification of rbCLD and

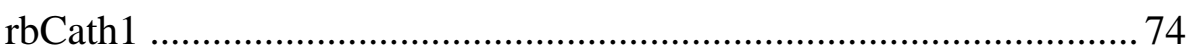

Figure 3.3 Removal of His. Tag from rbCLD and rbCath1 ....................................... 76

Figure 3.4 LPS-binding activity of rbCLD and rbCath1 f......................................... 77

Figure 3.5 Photomicrographs of microbial agglutination after 1 hour treatment ........... 80

Figure 3.6 Photomicrographs of microbial agglutination after 3 hours treatment.......... 81

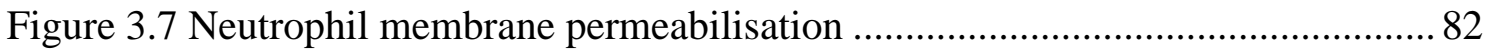

Figure 3.8 Cleavage of rbCath1 by neutrophil elastase ............................................ 84

Figure 3.9 Peptide mass fingerprint data after post-source decay for the $3 \mathrm{kDa}$

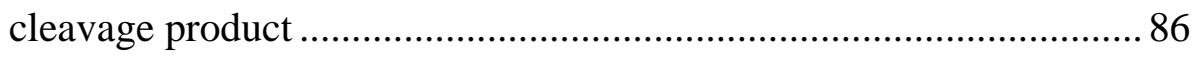

Figure 3.10 LC chromatograms of stimulated neutrophil culture media ........................ 87

Figure 3.11 The effect of the RITK peptide on bovine neutrophil respiratory burst ...... 89

Figure 4.1 Primary screen of neutrophil function 1 .................................................. 100

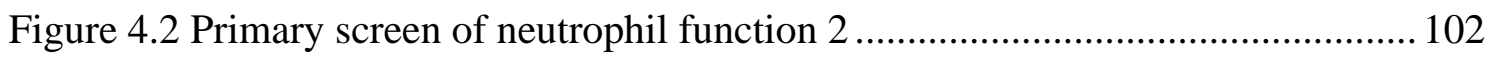

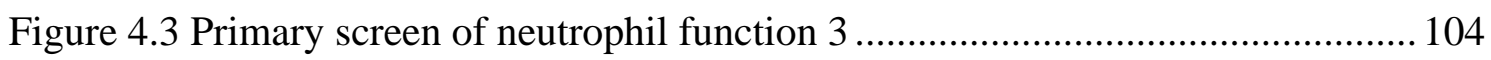

Figure 4.4 Survival of naïve neutrophils after linear Bac1, Bac5 or BMAP-34

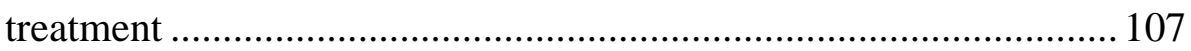

Figure 4.5 Survival of activated neutrophils after linear Bac1, Bac5 or BMAP-34

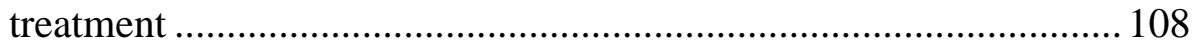

Figure 4.6 Neutrophil migration induced by linear Bac1, Bac5 or BMAP-34 ............ 109

Figure 4.7 Neutrophil granule release after 6 hours of linear Bac1, Bac5 or BMAP-34 treatment

Figure 4.8 Neutrophil respiratory burst after linear Bac1, Bac5 or BMAP-34 treatment 
Figure 4.9 Phagocytosis during linear Bac1, Bac5 or BMAP-34 treatment

Figure 4.10 Calcium mobilisation during linear Bac1, Bac5 or BMAP-34 treatment

Figure 4.11 LPS-binding activity of bovine cathelicidin AMPs 118

Figure 4.12 Summary of the effects of linear Bac1, Bac5 and BMAP-34 on neutrophil function in vitro

Figure 5.1 Confocal microscopy of Bac5-FITC-treated neutrophils 128

Figure 5.2 Flow cytometry analysis of Bac5-FITC-treated neutrophils

Figure 5.3 Flow cytometry analysis of Bac5-induced intracellular ROS production ... 131

Figure 5.4 Confocal microscopy of Bac5 and intracellular ROS colocalisation 132

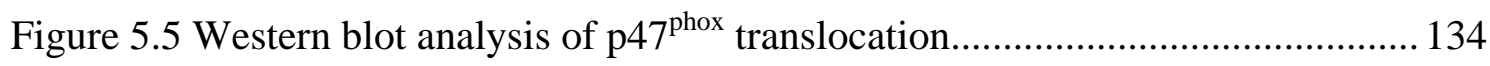

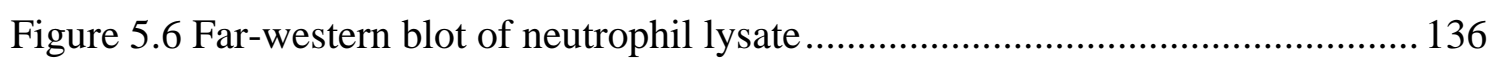

Figure 5.7 Immunopurification of cellular $\beta$-actin and far-western blot ..................... 139

Figure 5.8 Co-immunopurification of cellular $\beta$-actin and Bac5 ................................. 141

Figure 5.9 Co-immunopurification of purified $\beta$-actin and Bac5 .............................. 143

Figure 5.10 Confocal microscopy of Bac5 and $\beta$-actin colocalisation ........................... 144

Figure 5.11 Confocal microscopy of Bac5, intracellular ROS and $\beta$-actin colocalisation .

Figure 5.12 Flow cytometry analysis of PMA-induced intracellular ROS production in the presence of Bac5

Figure 5.13 Western blot analysis of $\mathrm{p} 47^{\mathrm{phox}}$ translocation in activated cells 148

Figure 5.14 Confocal microscopy of Bac5, intracellular ROS and $\beta$-actin 149

Figure 5.15 Summary of the potential mechanisms of Bac5-induced ROS production in bovine neutrophils

Figure 6.1 Summary of the roles of bovine cathelicidins in host defence

Figure A1.1 Analysis of false-positives in bovine cytokine ELISAs . 195

Figure A1.2 Test of ELISA blocking reagents 196 


\section{List of Tables}

Table 1.1 Examples of cathelicidin AMP classification and diversity .........................25

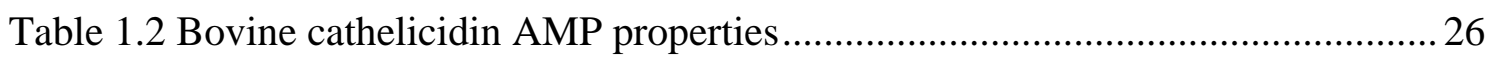

Table 1.3 Summary of the immunomodulatory activity of bovine and human

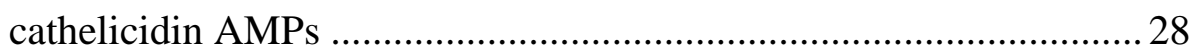

Table 1.4 Summary of the immunomodulatory effects of other cathelicidin AMPs ...... 29

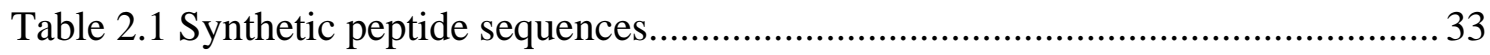

Table 2.2 Mini polyacrylamide gel composition ..................................................... 41

Table 2.3 Combinations of cellular $\beta$-actin and Bac5-FITC co-immunopurification..... 60

Table 2.4 Combinations of purified $\beta$-actin and Bac5-FITC co-immunopurification .... 60

Table 2.5 Confocal microscopy excitation and emission settings .................................65

Table 3.1 Multiple C-terminal sequence alignment of bovine cathelicidins. .................69

Table 3.2 Summary of mass spectrometry analysis of rbCLD …................................ 75

Table 3.3 Microbial agglutination scores after 1 hour treatment ................................... 79

Table 3.4 Microbial agglutination scores after 3 hours treatment ................................ 79

Table 4.1 Properties of the selected bovine cathelicidin AMPs .................................... 97

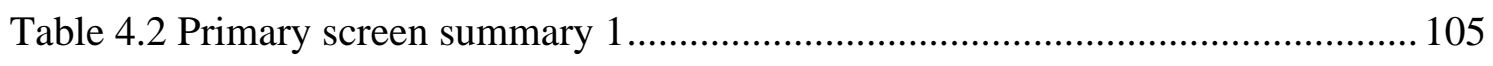

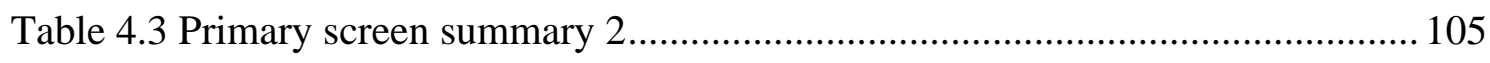

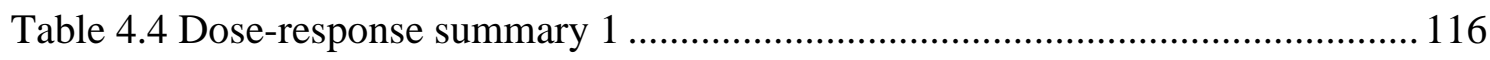

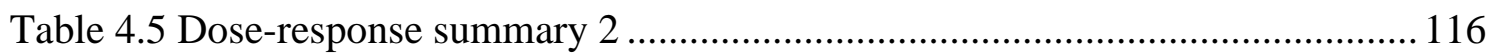

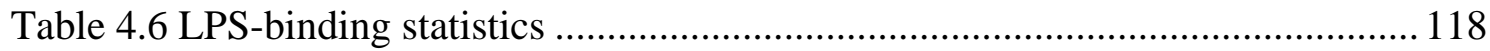

Table 4.7 Examples of cathelicidin AMPs that bind LPS .......................................... 123

Table 5.1 Summary of the LC-MS/MS data for the Bac5-FITC far-western blot........ 136 


\section{Abbreviations}

AMP

APE

APS

AU

$\mathrm{Bac}$

BCA

BMAP

BPI

BRD

BSA

$\mathrm{C} 5 \mathrm{aR}$

CAPS

CD

CGD

CHCA

CLD

CR

CV

DAPI

DNA

ECL

EDTA

ELISA

FBS

FcR

FITC

fPRL1

GM-CSF

hCAP-18

HRP

ICAM-1
Antimicrobial peptide

Antimicrobial peptide elicitor

Ammonium persulfate

Absorbance units

Bactenecin

Bicinchoninic acid

Bovine myeloid antimicrobial peptide

Bactericidal/permeability-increasing protein

Bovine respiratory disease

Bovine serum albumin

C5a receptor

$\mathrm{N}$-cyclohexyl-3-aminopropanesulfonic acid

Cluster of differentiation

Chronic granulomatous disease

alpha-cyano-4-hydroxycinnamic acid

Cathelin-like domain

Complement receptor

Column volumes

4',6-diamidino-2-phenylindole

Deoxyribonucleic acid

Enzyme-linked chemiluminescence

Ethylenediaminotetraacetic acid

Enzyme-linked immunosorbent assay

Fetal bovine serum

Fc receptor

Fluorescein isothiocyanate

Formyl peptide receptor-like-1

Granulocyte-macrophage colony-stimulating factor

Human cationic antimicrobial peptide-18

Horseradish peroxidase

Intercellular adhesion molecule-1 
IL

IMAC

INF $\gamma$

IPTG

JD

$\mathrm{KLH}$

LB

LBP

LC-MS/MS

LPS

LTA

MALDI-TOF/TOF Matrix-assisted laser desorption-(tandem) time-of-flight mass spectrometry

mCD14

MCP

MCP-1

MEC

MFI

MIC

MOPS

MOWSE

MPO

MQ. $\mathrm{H}_{2} \mathrm{O}$

MR

mRNA

MS

NADPH

$\mathrm{NF}-\kappa \mathrm{B}$

NMR

ObG

$\mathrm{OD}_{600}$

PAMP

PBS

Interleukin

Immobilised metal ion affinity chromatography

Interferon gamma

Isopropyl beta-D-1-thiogalactopyranoside

Johne's disease

Keyhole limpet haemocyanin

Luria broth

LPS binding protein

Liquid chromatography-(tandem) mass spectrometry

Lipopolysaccharide

Lipoteichoic acid

Membrane cluster of differentiation 14

2-methacryloxyethyl phosphorylcholine

Monocyte chemoattractant protein-1

Mammary epithelial cell

Median fluorescence intensity

Minimal inhibitory concentration

3-(N-morpholino)propanesulfonic acid

Molecular weight search

Myeloperoxidase

Milli-Q water

Mannose receptor

Messenger ribonucleic acid

Mass spectrometry

Nicotinamide adenine dinucleotide phosphate

Nuclear factor-kappa B

Nuclear magnetic resonance

Octyl-beta-D-glucopyranoside

Optical density at $600 \mathrm{~nm}$

Pathogen associated molecular pattern

Phosphate buffered saline 


\begin{tabular}{|c|c|}
\hline PCR & Polymerase chain reaction \\
\hline Rac & Rho-related C3 botulinum toxin substrate \\
\hline $\mathrm{RBC}$ & Red blood cell \\
\hline rbCath1 & Recombinant bovine Cath1 \\
\hline rbCLD & Recombinant bovine cathelin-like domain \\
\hline rEK & Recombinant enterokinase \\
\hline RMSD & Root-mean-square deviation \\
\hline ROS & Reactive oxygen species \\
\hline SCC & Somatic cell count \\
\hline sCD14 & Soluble cluster of differentiation 14 \\
\hline SDS-PAGE & $\begin{array}{l}\text { Sodium dodecyl sulphate-polyacrylamide gel } \\
\text { electrophoresis }\end{array}$ \\
\hline SLB & Sample loading buffer \\
\hline SOC & Super optimal broth with catabolite repression \\
\hline TBS & Tris buffered saline \\
\hline TCA & Trichloroacetic acid \\
\hline TCII & Transcobalamin II \\
\hline TE & Tris EDTA \\
\hline TEMED & Tetramethylethylenediamine \\
\hline TFA & Trifluoroacetic acid \\
\hline TLR & Toll-like receptor \\
\hline TMB & 3,3',5,5'-tetramethylbenzidine \\
\hline $\mathrm{TNF} \alpha$ & Tumour necrosis factor alpha \\
\hline Tris & Tris(hydroxymethyl)aminomethane \\
\hline TRITC & Tetramethylrhodamine isothiocyanate \\
\hline WST-1 & Water soluble tetrazolium salt- 1 \\
\hline
\end{tabular}


Chapter 1: General Introduction 


\subsection{Overview}

The past 50 years has seen a rapid increase in all aspects of agricultural production to meet global demand. Modern livestock farming techniques utilise large herd sizes which maximise production and returns. However, intensive livestock farming methods can potentially suffer from a greater incidence of disease. The scope of diseases affecting agricultural cattle cause significant global economic losses in terms of detection, treatment, control, eradication and most notably, loss of production.

Due to the constant exposure of cattle to environmental pathogens, common prevalent contagious bacterial infections are generally accrued by cattle at mucosal surfaces exposed to the external environment. The most notable areas susceptible to disease are the intestinal tract, the respiratory tract and lungs, and the mammary gland. Understanding how the host responds and defends itself at these mucosal surfaces, in terms of the etiology and progression of disease, could provide opportunities through which improved approaches to disease management can be developed.

This general introduction provides brief examples of mucosal bacterial diseases and their impact on the agricultural industry. It then describes in detail the innate immunological strategies used by bovine to defend against infection at mucosal surfaces via anatomical, cellular and molecular mechanisms. Further to this, a description of the cathelicidins is provided, with particular attention to cathelicidin structure, localisation and processing, and known functions in the innate immune response. 


\subsection{Mucosal infections in bovine}

Johne's disease (JD) is a chronic infection of the small intestine caused by Mycobacterium avium subspecies paratuberculosis which results in diarrhoea, weight loss, decreased milk production and morbidity in ruminants $[1,2]$. The herd prevalence of JD in the dairy industry of the United States is approximately $40 \%$, with estimated annual losses as high as US\$250 million (1999 estimates [3,4]). A recent report suggests the prevalence of JD in New Zealand beef cattle is approximately $31 \%$ and as high as $73 \%$ in sheep and deer [5] although a majority of these animals do not exhibit any clinical symptoms of the disease. M. paratuberculosis also persists in wild life and survives in soil hence attempts at eradicating the disease have been unsuccessful. Current research focuses on developing reliable detection methods and control programs to prevent the spread of infection.

Bovine respiratory disease (BRD) is of significant importance in the beef cattle industry, particularly in North America. The disease is caused by infection of the lungs and respiratory tract by Mannheimia haemolytica, Pasteurella multocida, Histophilus somni or Mycoplasma bovis [6]. Predisposing factors include viral infections, transportation and weather conditions [7]. Total economic losses have been estimated at approximately US $\$ 14,000$ annually per 1,000 animals with $14 \%$ incidence of BRD (2006 estimate [8]).

In dairy cattle the most prevalent and costly disease is mastitis, an infection of the mammary gland. In New Zealand, mastitis of dairy cows is a significant animal welfare and production issue, with associated annual losses estimated at \$180 million (2006 calculation [9]). Mastitis causes reduced milk production (both short and long term), as well as costs associated with animal treatment, disposal of milk from antibiotic treated animals and penalty payments for herds with high levels of mastitis. Frequent mastitiscausing pathogens include Escherichia coli, Streptococcus uberis, Streptococcus dysgalactiae, Streptococcus agalactiae and Staphylococcus aureus [10]. Multiple factors contribute to mastitis susceptibility in bovine including the age of cattle, genetic background, the stage of lactation, milking practices, nutrition and climate [11]. 


\subsection{Host defence in bovine}

Protection from pathogens relies on multiple mechanisms that include anatomical and innate cellular and humoral defence systems [12-15].

\subsubsection{Anatomical defence}

The nasal and oral mucosa provides the first line of defence against respiratory and gastrointestinal pathogen entry by trapping opportunistic bacteria. In the respiratory tract, the action of cilia on epithelial cells acts to sweep pathogens out and away from the lungs. In the gastrointestinal tract, peristalsis helps to remove pathogens and the intestinal mucosa allows colonisation by commensal bacteria, which helps to prevent colonisation by pathogens through competition for nutrients and excretion of compounds that are toxic to pathogens [16].

In the mammary gland the teat end is the first line of defence against the entry of pathogens (Figure 1.1). Sphincter muscles keep the streak canal tightly closed until milking. This creates a physical barrier which obstructs invading pathogens [17]. The streak canal is lined with keratin forming a mesh-like structure containing antimicrobial agents that assist in preventing entry of pathogens [18]. Streak canal length and diameter correlate with incidence of mastitis, with shorter wider canals being more susceptible to infection [19]. At the entry to the teat cistern is a structure called Furstenberg's rosette which hosts an enhanced population of leukocytes [20]. These cells are capable of leaving the teat wall to neutralise pathogens.

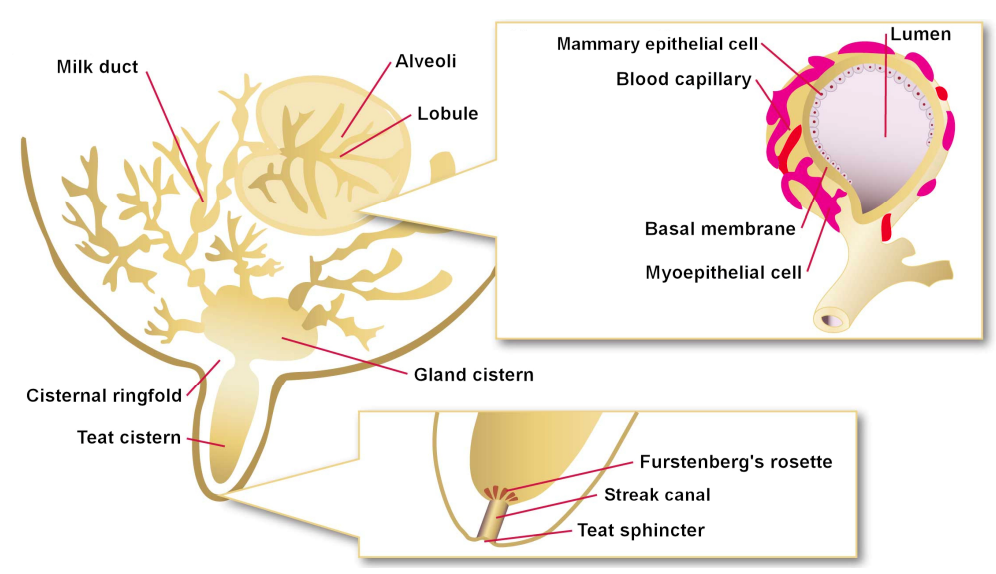

Figure 1.1 Structure of the bovine mammary gland

This diagram has been adapted and adjusted from Thayer, 2007 [21]. 


\subsubsection{Innate cellular defence}

Myeloid-derived cells are the major component of cellular innate immunity. These include macrophages, dendritic cells and neutrophil granulocytes that recognise and signal pathogen exposure to initiate immune protection and eliminate pathogens (Figure 1.2).

Infection is detected by pathogen recognition receptors on macrophages, dendritic cells and granulocytes. These receptors include a diverse range of Toll-like receptors (TLRs), complement receptors (CRs), dectin receptors, mannose receptors (MRs), immunoglobulin (Ig) receptors (FcRs) and cluster of differentiation (CD) cell surface molecules. TLRs differentially recognise common exogenous pathogen components such as lipopolysaccharide (LPS, also known as endotoxin), lipoteichoic acid (LTA), foreign double-stranded deoxyribonucleic acid (DNA), CpG DNA and flagellin [22].
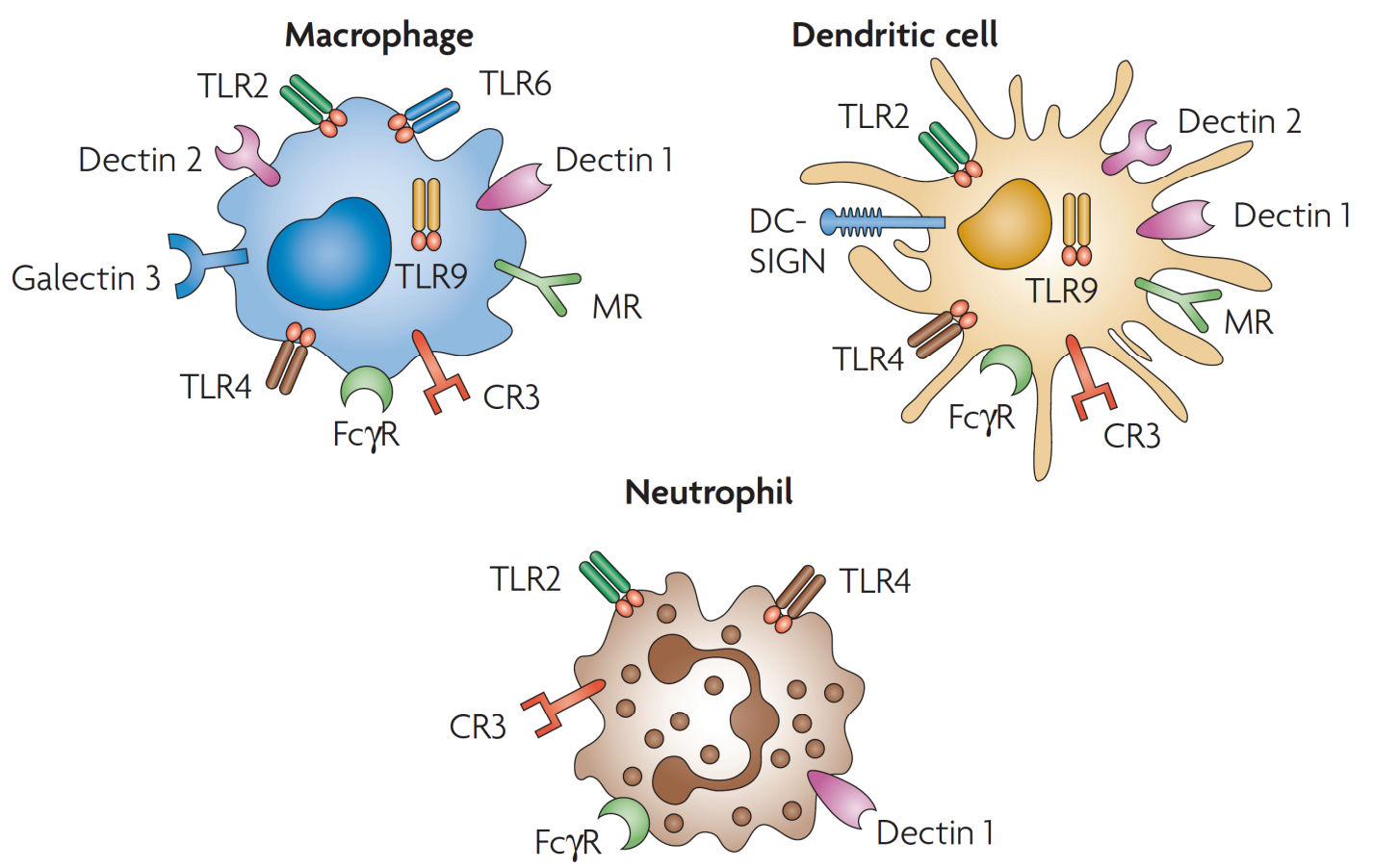

Figure 1.2 Myeloid-derived innate immune cells and associated receptors

This diagram has been adapted from Brown et al, 2008 [23]. 
CRs recognise endogenous proteins of the complement cascade and exogenous yeast cell wall glucans such as zymosan [24]. Dectin receptors recognise microbial components and endogenous ligands [25]. Igs bind to microbes and microbial molecules forming antigen-antibody complexes which are recognised by FcRs. FcRs form a large family of receptors which are classified based on the type of Ig molecule they bind [26]. CD molecules are commonly associated with cells involved in immunity which recognise antigens, e.g. CD4 and CD8 T cells. However, cells involved in innate immunity express CD14 which assists in recognition of LPS [24].

As part of the innate immune response, recognition of pathogens by macrophages and dendritic cells results in chemokine and cytokine signalling to recruit granulocytes from the circulation. These granulocytes are predominantly neutrophils. Granulocyte recruitment involves adhesion to vascular walls, diapedesis, and chemotaxis toward inflammatory foci. Infiltration is dramatic. For example, during mastitic infection myeloid cell numbers increase 1000-fold within 24 hours, with neutrophils constituting approximately $95 \%$ of total cells infiltrating the mammary gland [27]. This influx of cells into the mammary gland results in an increase in somatic cell count (SCC), a parameter routinely assessed by farmers and veterinarians to monitor levels of subclinical mastitis in milking herds. Importantly, the recruitment and activation status of neutrophils is critical in containing and eliminating pathogens during infection. For this reason bovine neutrophils are the principal cell type studied in this thesis.

Neutrophil stimulation induces a range of functions designed to defend against pathogens, including respiratory burst, phagocytosis and degranulation. Degranulation releases AMPs, proteases, lipases and glycosidases into the extracellular medium that constitute part of the humoral innate immune response [28] (section 1.3.3).

\subsubsection{Adhesion and diapedesis}

The epithelium functions as a dynamic barrier. Release of inflammatory cytokines from resident immune cells and epithelial cells cause endothelial cells in the vicinity to increase expression of E-selectin and P-selectin adhesion molecules, allowing neutrophils to adhere to blood vessel walls [29]. Neutrophils upregulate macrophage-1 antigen, which increases interaction with intercellular adhesion molecule-1 (ICAM-1) 
on endothelial cells, allowing neutrophils to migrate across the endothelium [14]. Cell to cell interactions between epithelial cells are modulated by cytokines and in the mammary gland milk electrolyte content also modulates endothelial cell junctions [30]. This allows neutrophils to pass without permanently disrupting cell to cell contacts.

\subsubsection{Chemotaxis}

Chemoattractant concentration gradients allow neutrophils to migrate from the endothelium to the site of infection. Inflammatory environments often contain multiple chemoattractants, therefore neutrophils utilise an intracellular signalling hierarchy where end-target chemoattractants such as formyl peptides and complement C5a dominate over intermediary chemoattractants such as interleukin (IL)-8 and leukotriene $\mathrm{B}_{4}$ [31]. Although neutrophils possess multiple receptors for different chemokines, the exact types of receptors can differ between species resulting in different responses to common stimuli. Several examples are provided below which highlight the differences in neutrophil responses to chemotactic stimuli, not only between species, but also within species exhibiting polymorphisms for particular receptors.

For instance, human neutrophils express formyl peptide receptor-like-1 (fPRL1) receptors that are activated by bacterial formyl peptides [32] and show enhanced chemotaxis in the presence of LPS [33]. Bovine neutrophils do not recognise formyl peptides [34] nor exhibit increased chemotaxis in the presence of LPS [35]. The anaphylatoxins $\mathrm{C} 5 \mathrm{a}$ and $\mathrm{C} 5 \mathrm{a}^{\mathrm{desArg}}$ are produced by the complement cascade [36]. Chemotactic responses to $\mathrm{C} 5 \mathrm{a}$ and $\mathrm{C} 5 \mathrm{a}^{\text {desArg }}$ are comparable in bovine neutrophils, whereas human neutrophils are 20-50 times less responsive to C5a ${ }^{\text {desArg }}$ [37]. IL-8 is a potent chemoattractant produced by macrophages, epithelial cells and to a lesser extent neutrophils [38]. Bovine neutrophils express two IL-8 receptors which are homologous to those found in humans [39]. Accordingly, bovine neutrophils respond to recombinant human IL-8 [40]. However, polymorphisms in the bovine chemokine CXCR1 receptor confer different responses to bovine IL-8 which impact on resistance to mastitis [41]. 


\subsubsection{Degranulation}

Neutrophil degranulation releases a number of humoral innate immune components, including cathelicidins and defensins. These humoral innate immune components are discussed in section 1.3.3.3. Degranulation also increases cell surface expression of receptors and oxidase complexes derived from granule membranes. The process of neutrophil degranulation is thought to occur in a sequential manner [42] and is regulated by the actin cytoskeleton [43]. Neutrophil secretory vesicle release occurs during adherence to endothelia, which relocates vesicle membrane-bound receptors to the neutrophil plasma membrane, thereby altering endothelial membrane protein expression. Different granule subsets are then released during diapedesis, migration and phagocytosis.

The development of granule subsets occurs from the promyelocyte stage of neutrophil maturation [44] and is significantly different in bovine and human neutrophils. Both species produce primary (azurophil) and secondary (specific) granule subsets with similar contents. However, bovine neutrophils also produce novel large granules [45] which store the bovine cathelicidins. In contrast the human homologue human cathelicidin antimicrobial peptide-18 (hCAP-18) is stored in the secondary granules [46] and human neutrophils produce tertiary, gelatinase-containing granules [47].

\subsubsection{Respiratory burst}

Respiratory burst is the process of producing ROS to kill pathogens and initiate cellular responses to infection. This is achieved through the formation of a membrane coupled NADPH oxidase complex which converts molecular oxygen to superoxide radical using NADPH and molecular oxygen as substrates (Figure 1.3) [48]. Receptor activation via pathogens, chemoattractants or other endogenous molecules activates intracellular kinases which phosphorylate cytosolic subunits $\mathrm{p} 40^{\text {phox }}, \mathrm{p} 47^{\text {phox }}$ and $\mathrm{p} 67^{\text {phox }}$ of the NADPH oxidase complex. These subunits then translocate to plasma or phagosome membranes, along with Rho-related C3 botulinum toxin substrate (Rac), and bind to the transmembrane subunits $\mathrm{p} 22^{\text {phox }}$ and gp $91^{\text {phox }}$, completing the oxidase assembly. 
Chronic granulomatous disease (CGD) is a condition resulting from the absence of a functional NADPH oxidase due to mutations in either of four oxidase subunits [49]. The disease manifests as increased susceptibility to infection, particularly in the lung, skin, liver and lymph nodes. Although not a condition found in cattle, the lack of a functional oxidase complex highlights the importance of oxygen-dependent killing mechanisms in immune cells.

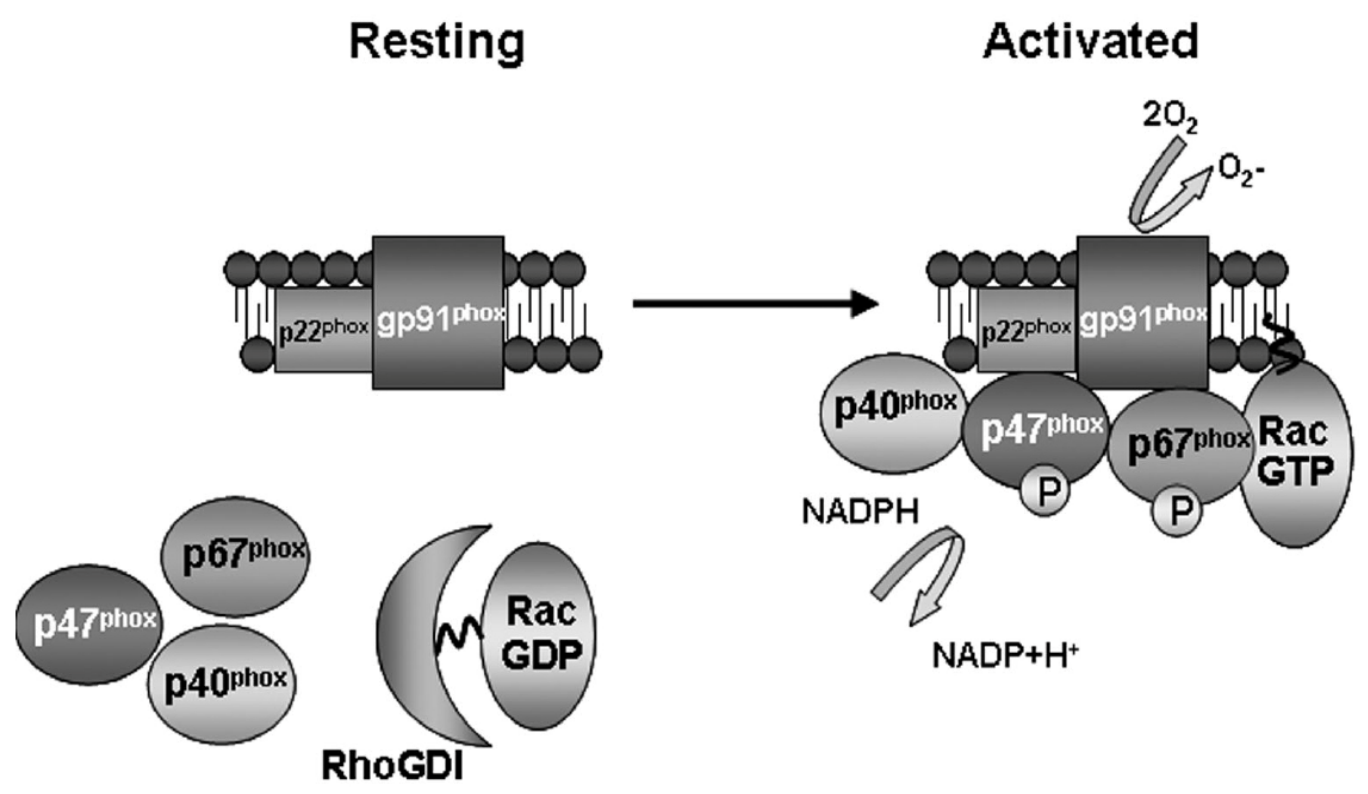

Figure 1.3 The NADPH oxidase complex in resting and activated cells

This diagram was taken from Wilkinson and Landreth, 2006 [50]. GDP, guanosine diphosphate; GTP, guanosine triphosphate; $\mathrm{P}$, phosphate. 


\subsubsection{Phagocytosis}

Neutrophils can function as phagocytes in tissues for one to two days [51]. Phagocytosis is mediated by $\mathrm{Fc}$ receptors and CRs. Fc receptors recognise antibody-antigen complexes on pathogens and foreign bodies. CR1 and CR3 respectively recognise C3bopsonised or iC3b-opsonised pathogens and foreign particles [52]. In addition to clearing bacteria, the process of phagocytosis can stimulate the release of cytokines and chemokines that contribute to the progression of the immune response [53-55].

\subsubsection{Cellular functions in the mammary gland and milk}

It is speculated that the non-tissue environment of milk renders many neutrophil innate immune functions less effective. Diapedesis across mammary epithelium is known to reduce the bactericidal activity of neutrophils [56]. This includes respiratory burst activity and phagocytic capacity, which are diminished due to the ingestion of fat globules and the associated loss of pseudopodia [56,57]. However, during infection the composition of milk dramatically changes [58]. These studies were carried out in normal milk and may not accurately reflect the in vivo functionality of cellular defence during infection. Nevertheless, cellular components of innate immunity are critical in containing and eliminating pathogens but also rely on components of humoral innate immunity for efficient host defence. 


\subsubsection{Innate humoral defence}

Humoral components of innate immunity include proteins and peptides capable of directly neutralising pathogens, or proteins and peptides that aid cellular elimination of pathogens. These components are generally divided into two arms - sensing molecules, i.e. molecules that bind to pathogens and enhance recognition of pathogens by host defence cells, or effecting molecules i.e. molecules that initiate phagocytosis by phagocytes or directly neutralise pathogens by inducing cell death [28]. Additionally, some host defence peptides are also able to prevent recognition of pathogens [59] thereby regulating proinflammatory responses, and should be considered as a third arm of innate humoral defence.

\subsubsection{Complement system}

The complement system is a series of liver-derived proteins ( $\mathrm{C} 1$ to $\mathrm{C} 9$ ) found in blood serum [60]. Activation of complement occurs via several mechanisms but most notably through pathogen recognition. Activation and successive cleavage of precursor proteins generate components with diverse immunomodulatory effects. Cleavage of C3 generates the opsonins $\mathrm{C} 3 \mathrm{~b}$ and $\mathrm{iC} 3 \mathrm{~b}$ which initiate phagocytosis of opsonised pathogens. $\mathrm{C} 5$ is cleaved to generate $\mathrm{C} 5 \mathrm{a}$, a potent anaphylatoxin. $\mathrm{C} 5 \mathrm{a}$ is rapidly converted to $\mathrm{C} 5 \mathrm{a}^{\text {desArg }}$ through the removal of the $\mathrm{C}$-terminal arginine by serum carboxypeptidase $\mathrm{N}$ [61]. In humans $\mathrm{C} 5 \mathrm{a}^{\text {desArg }}$ has significantly less activity than C5a whereas in bovine both forms have equivalent activity [37].

C5a activates cellular responses via the seven-transmembrane domain, G-proteincoupled $\mathrm{C} 5 \mathrm{a}$ receptor $(\mathrm{C} 5 \mathrm{aR})$ which is highly expressed on bovine neutrophils [62]. In comparison to the human C5aR and C5aRs from several other species, bovine and ovine C5aRs appear to have unique amino acid substitutions in loop regions critical for C5a recognition [62]. This potentially allows recognition and activation by both C5a and C5 $\mathrm{a}^{\text {desArg }}$, and may compensate for the absence of fPRL receptors on bovine neutrophils [34]. Activation by C5a induces chemotaxis, degranulation and respiratory burst [63]. Components C5b to C9 form the membrane attack complex [60]. Formation of this complex is the end-point of complement activation and results in permeabilisation of the target membrane, lysis and cell death. 


\subsubsection{Epithelial cell secretions and milk}

Epithelial cells secrete a number of chemoattractants such as IL-8 and monocyte chemotactic protein-1 (MCP-1) and upregulate expression of ICAM-1 to promote neutrophil migration during infection [64]. They also secrete proinflammatory cytokines such as tumour necrosis factor alpha (TNFa), granulocyte-macrophage colonystimulating factor (GM-CSF), IL-1 $\alpha$ and IL-1 $\beta$ in a rapid but transient manner in response to pathogens [64]. The repertoire of antimicrobial secretions produced by both airway and gastrointestinal epithelial cells include $\beta$-defensin, lysozyme and phospholipase A2 [65,66]. Airway epithelia also secrete lactoferrin [65], and intestinal epithelia also secrete bactericidal/permeability-increasing protein (BPI) [66]. In contrast to human epithelial cells, bovine epithelial cells, including mammary epithelial cells (MECs), show negligible constitutive expression of cathelicidins [67-69].

The MECs lining the alveoli of the mammary gland either transport or synthesize a number of innate immune components, secreting them into the alveolar lumen (Figure 1.1). Hence milk itself is considered part of the innate immune system [12]. Acquired immune system components such as immunoglobulins and secretory component also accumulate in milk via transcellular transport through MECs from the blood to the lumen, while solutes accumulate in milk via paracellular transport [70]. MECs synthesise a number of host defence proteins such as lactoferrin, $\beta$-defensin and LPS-binding protein (LBP) (section 1.3.3.3) [12,13]. During secretion of these components the apical membranes of MECs are released, forming the milk fat globule membrane component of milk [71]. The milk fat globule membrane contains xanthine oxidase, sphingolipids and numerous other immune factors [58]. MECs are also capable of pathogen recognition and express several different TLRs recognising lipopeptides, peptidoglycan, LPS and foreign DNA [72].

Digestion of ingested milk by neonates produces a variety of humoral peptides generated from larger milk proteins by proteolytic degradation, including antihypertensive peptides, antithrombotic peptides and caseinophosphopeptides [73]. These peptides prevent local increases in blood pressure, prevent clotting of blood, and form complexes with calcium which aid intestinal absorption, respectively. Peptide 
derivatives of $\alpha$ s1-casein, $\beta$-casein, $\kappa$-casein, $\alpha$-lactalbumin and $\beta$-lactoglobulin also have multiple immune-modulating roles in the digestive tract $[73,74]$.

\subsubsection{Neutrophil secreted proteins and peptides}

Secretion of proteins and peptides by neutrophils occurs through secretory vesicle release and degranulation. Secretory vesicle release upregulates surface expression of membrane bound CD14 (mCD14) [75] which recognises LPS-LBP complexes, resulting in cellular activation. During mastitis mCD14 is cleaved from the membrane by proteases to release soluble CD14 (sCD14) which has high affinity for LPS [76]. The CD14-LPS complex can activate TLRs on epithelial cells which release chemokines to enhance neutrophil recruitment.

Primary bovine neutrophil granules contain myeloperoxidase (MPO) which catalyses the formation of bactericidal hypohalous acids and nitrogen oxides [48]. In contrast to patients with CGD (NADPH oxidase deficiency), MPO deficiency does not result in higher incidence of infection due to parallel mechanisms of bacterial killing [77]. These mechanisms utilise lactoferrin, BPI, cathelicidin and defensin mediated bacterial killing.

Lactoferrin is stored in the secondary granules of neutrophils. Lactoferrin has bacteriostatic activity through iron sequestration and bactericidal activity through direct interaction with bacteria [78]. BPI shares homology to LBP but has significantly higher affinity for LPS $[79,80]$, and like lactoferrin, has direct bactericidal activity. Lysozyme is a component of primary, secondary and tertiary granule types in human neutrophils [47] but is absent in bovine, caprine and ovine neutrophils [81].

The two major families of antimicrobial peptide released by neutrophils are defensins and cathelicidins. The main focus of this thesis is the cathelicidins which are introduced in section 1.4. Defensins are small cysteine-rich cationic proteins found in immune cells and nearly all epithelial cells. There are three structural subfamilies termed $\alpha-, \beta-$, and $\theta-$ defensin [82]. Various physiological stimuli mobilise preformed defensins from neutrophil granules or upregulate expression in tissues. The majority of defensins have antimicrobial activity. Their effects on mammalian cells are numerous, including chemotaxis and degranulation of mast cells, recruitment and maturation of dendritic 
cells, chemotaxis of monocytes and $\mathrm{T}$ cells, mitogenesis of epithelial cells, neovascularisation and wound healing [83]. 


\subsection{Cathelicidins}

The cathelicidins are a family of diverse host defence proteins found in vertebrates (Figure 1.4). This section will focus on cathelicidin proteins and the cathelin-like domain (CLD). The cathelicidin antimicrobial peptide (AMP) domain will be discussed in more detail in section 1.4.6.

\subsubsection{History}

The discovery of oxygen-independent killing mechanisms in bovine neutrophil phagosomes led to the identification of bactericidal polypeptides localised to the unique large granules. The antibiotic activity of these peptides was broad and efficient, killing Gram-positive and Gram-negative bacteria at concentrations of less than $100 \mu \mathrm{g} / \mathrm{ml}$ [45,84]. Purification of large granule contents allowed identification and characterisation of several cationic peptides with bactericidal activity at micromolar concentrations. The peptides were named 'bactenecins' $[85,86]$.

Full length complementary DNA clones of bactenecin peptides revealed a conserved $\mathrm{N}$-terminal pro-sequence of approximately $11.5 \mathrm{kDa}$ which had $66 \%$ amino acid sequence identity to cathelin, a cathepsin L inhibitor from porcine neutrophils [87]. Hence this conserved domain was termed the 'cathelin-like domain'. The novel protein has since been identified in many mammalian species and other vertebrates. The protein family was named 'cathelicidins'. 
A

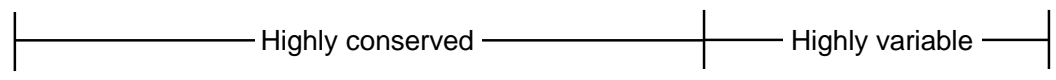

\begin{tabular}{|c|c|c|}
\hline Signal peptide & Cathelin-like domain (CLD) & AMP domain \\
\hline & & \\
& & \\
& &
\end{tabular}

B
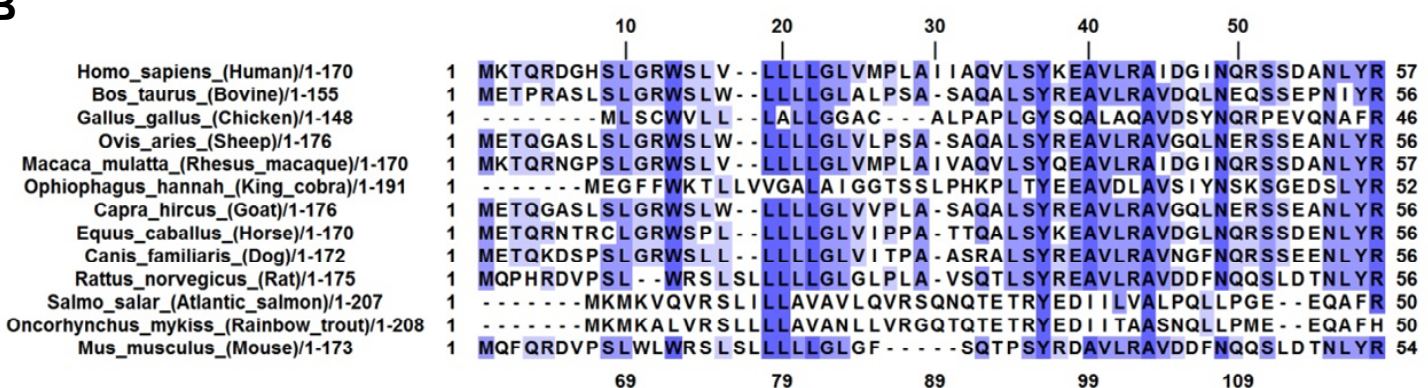

Homo_sapiens_(Human)/1-170

Bos_taurus_(Bovine)/1-155

Gallus_gallus_(Chicken)/1-14 Ovis_aries_(Sheep)/1-176 Macaca_mulatta_(Rhesus_macaque)/1-170 Ophiophagus_hannah_(King_cobra)/1-191 Capra_hircus_(Goat)/1-176 Equus_caballus_(Horse)/1-170 Canis_familiaris_(Dog)/1-172 Rattus_norvegicus_(Rat)/1-175 Salmo_salar_(Atlantic_salmon)/1-207 Oncorhynchus_mykiss_(Rainbow_trout)/1-208 Mus_musculus_(Mouse)/1-173

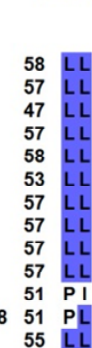

$$
69 \quad 79
$$

89

$$
99
$$

109
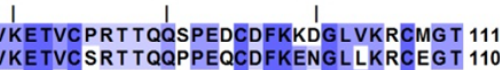
ADP - - EPGPNVQLSSLHNLNF TIMETRCQARSGAQLDSCEFKEDGLVKDCAAP 100 . - APNDEVDPGTRKPVSFT TKETVCPRTTQQPPEECDFKENGL LKQCVGT 110 LDP - - RP TMDGDPDTPKPVSFTVKETVCPRT TQKSPEDCDFKEDGLVKRCVGT 11 EAVP - . P P EWDPLSESNQELNF T I KE TVCL VAEERSLEECDFQEDGA I MGC TGY 106 DP - - APNDEVDPGTRKPVSFT TKETVCPRT TQQPPEECDFKENGLVKQCVG T 110 DP - - LPKGDKDSDTPKPVSFMVKETVCPR I MKQTPEQCDFKENGLVKQCVGT 110 NS - - QPKGDEDPN I PKPVS FTVKETVCPKT TQQPLEQCGFKDNGLVKQCEG T110 DS - - - EPQGDEDPDTPKYVRFRVKETVCSKASQQLPEQCAFKEQGVVKQCMGT 110

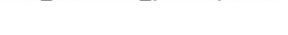

Homo_sapiens_(Human)/1-170 Bos_taurus_(Bovine)/1-155 Bos_taus_(Bovine)/1-155 Ovis_aries_(Sheep)/1-176 Macaca_mulatta_(Rhesus_macaque)/1-170 Macaca_mulatta_(Rhesus_macaque)/1-170
Ophiophagus_hannah_(King_cobra)/1-191 Capra_hircus_(Goat)/11-176 Equus_caballus_(Horse)/1-170 Equis familiaris Cans_toris Rattus Oncorhynchus_mykiss_(Rainbow_trout)/1-20

Mus_musculus_(Mouse)/1-173

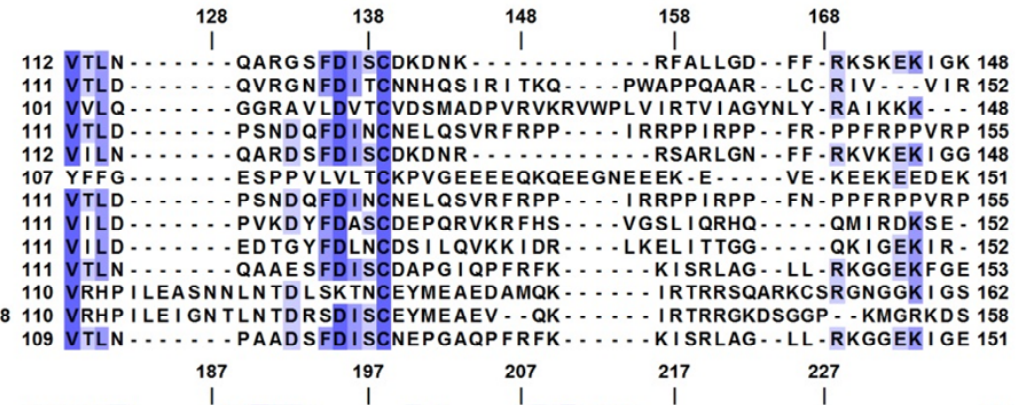

Homo_sapiens_(Human)/1-170 Bos_taurus_(Bovine)/1-155 Gallus_gallus_(Chicken)/1-148 Ovis_aries_(Sheep)/1-176 Macaca_mulatta_(Rhesus_macaque)/1-170 Ophiophagus_hannah_(King_cobra)/1-191 Capra_hircus_(Goat)/1-176 Equus_caballus_(Horse)/1-170 Canis_familiaris_(Dog)/1-172 Rattus_norvegicus_(Rat)/1-175 Salmo_salar_(Atlantic_salmon)/1-207 Oncorhynchus_mykiss_(Rainbow_trout) $/ 1$
Mus_musculus_(Mouse)/1-173 149 EFKRI . . . VQRIIKD . . . . . FLR . . . NLVPRTES . . . . . . . . . . . 170

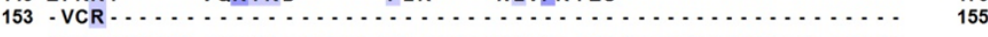

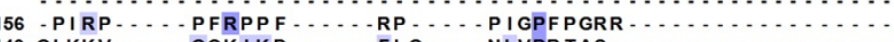
149 GLKKV . . . . GQKIKD . . . . . . FLG . . . . NLVPRTAS . . . . . . . . . . . . . 170 152 DQPRR - . . . . VKRFKK ……FFK … - KLKNSVKKRAKKFFKKPRVIGVSIPF - 191 156 -PFRP $\ldots .$. PFRPPF $\ldots \ldots$ RP $\ldots \ldots$ PIGPFPGRR $\ldots \ldots \ldots \ldots \ldots \ldots . . \ldots \ldots$

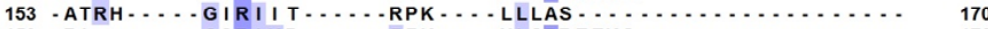
153 - RI - . . . GQR IKD - . . . - FFK - . - NLQPREEKS - . . . . . . . . . . 172

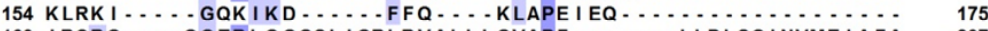
163 IRCRG.....GGTRLGGGSL IGRLRVALLLGVAPF ..........LLDLSQINVMEIAFA 207

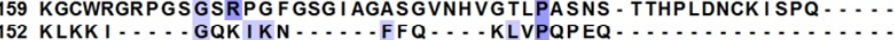

\section{Figure 1.4 Domain structure and multiple sequence alignment of cathelicidins}

(A) Domain structure of cathelicidins showing highly conserved and highly variable regions. (B) Multiple sequence alignment of cathelicidins from a range of species. Note the highly conserved cysteine amino acids at positions 93, 104, 115 and 139 which form the disulfide bonds in the CLD. Alignment created using Jalview 2 v2.8 [88]. 


\subsubsection{Gene structure}

Bovine chromosome 22 contains 11 distinct cathelicidin genes, seven of which are expressed [89]. The exon-intron organisation is highly conserved (Figure 1.5). Exons one to three encode the signal sequence and the highly conserved N-terminal CLD. Exon four encodes the cleavage site and AMP domain. The exon-intron locations avoid secondary structure elements and occur between amino acid codons. This is a typical feature of genes encoding extracellular immune proteins [90].

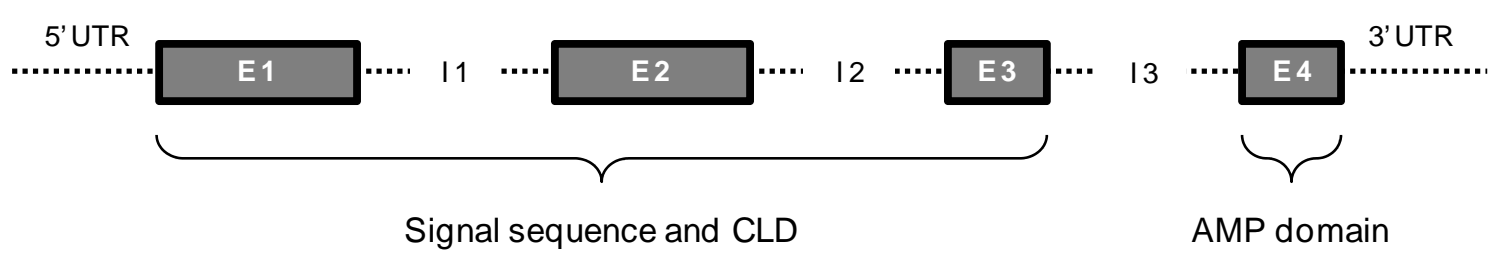

\section{Figure 1.5 Exon and intron structure of cathelicidin genes}

Exons one to three encode the signal sequence and CLD. Exon four encodes the AMP domain. E, exon; I, intron; UTR, untranslated region.

The cathelicidins evolved from a common ancestral cystatin scaffold and are phylogenetically classified in the cystatin superfamily of cysteine protease inhibitors. Organisation of exons one to three is identical in cystatins and cathelicidins suggesting cathelicidins arose through duplication of cystatin genes [90]. A proposed mechanism for the evolution of the AMP domain involves selection of splicing signals in the third intron of the gene, leading to read-through into the 3' untranslated region. Continual mutations in this region potentially led to the evolution of a protease cleavage site and significant diversity in the AMP domain [90]. The retention of this domain, despite the high diversity, shows each AMP domain has a valuable function in host defence.

Two natural variants of the cathelicidin-1 gene (CATHL1, accession: P22226 [91]) have been identified in cattle, indicating the alternative presence of either glutamine or arginine at position 128 of the protein sequence [87]. Sequence polymorphisms have also been identified in three cathelicidin genes (CATHL2, accession: P19660; CATHL5, accession: P54229; CATHL7, accession: P56425; [91]) across ten breeds of Bos taurus and Bos indicus species [92]. Seven nonsynonymous single nucleotide polymorphisms were identified. None were predicted to alter cathelicidin structure. 
Because an exact function for the CLD has not been defined, the change of amino acid sequence identified in these genes does not rule out alteration of an unknown function, which may involve modification of potential active site amino acids.

\subsubsection{Response elements}

Recognition sites for nuclear factors IL-6, nuclear factor-kappa B (NF- $\kappa$ B) and interferon gamma (IFN $\gamma$ ) response element have been identified in the 5' flanking region of bovine cathelicidin genes [89]. The presence of recognition sequences for immune related transcription factors suggest cathelicidin transcription could be regulated by cytokines during infection. Upregulation of bovine cathelicidin messenger ribonucleic acid (mRNA) has been reported in Mannheimia haemolytica infected cattle where elevated levels of Bac1 and indolicidin mRNA were detected in bronchoalveolar lavage fluid [93]. Bovine neutrophils exposed to Escherichia coli LPS are also reported to upregulate mRNA expression of Bac5 and BMAP-28 ex vivo [67]. However, it is unclear which transcription factors are responsible for upregulation in each example.

The promoter region of the human cathelicidin gene contains a vitamin D response element [94]. Expression of the gene can be induced by 1,25-dihydroxyvitamin D3 and three of its analogues, via binding to the transcription factor vitamin D receptor [95]. However, this mechanism only appears to operate in primates and is absent in murine, canine and bovine cathelicidin promoters [96].

\subsubsection{Protein structure}

Cattle express seven cathelicidins. The globular CLDs have $47.8 \%$ sequence identity with 55 identical positions, but vary greatly in their AMP domains. A structure-based sequence alignment of the cathelicidin family shows conservation in secondary structure elements and disulfide bond location [97]. Porcine protegrin-3 shares at least $65.8 \%$ sequence identity with bovine cathelicidins [91]. The structure of protegrin-3 was determined by nuclear magnetic resonance (NMR) spectroscopy and X-ray crystallography using chicken cystatin as a model [97,98]. The fold was revealed to have an N-terminal $\alpha$-helix partially wrapped by a four-stranded $\beta$-sheet which buries 
hydrophobic residues in the core of the protein. Two highly conserved disulfide bonds are essential for structural integrity [98].

More recently, the structure of the human CLD was solved by x-ray crystallography [99] (Figure 1.6). The human CLD shares 63.4\% sequence identity with protegrin-3 and the X-ray structures overlap with $0.88 \AA$ root-mean-square deviation (RMSD) for 81 of the $\mathrm{C} \alpha$ carbon backbone atom pairs.

A
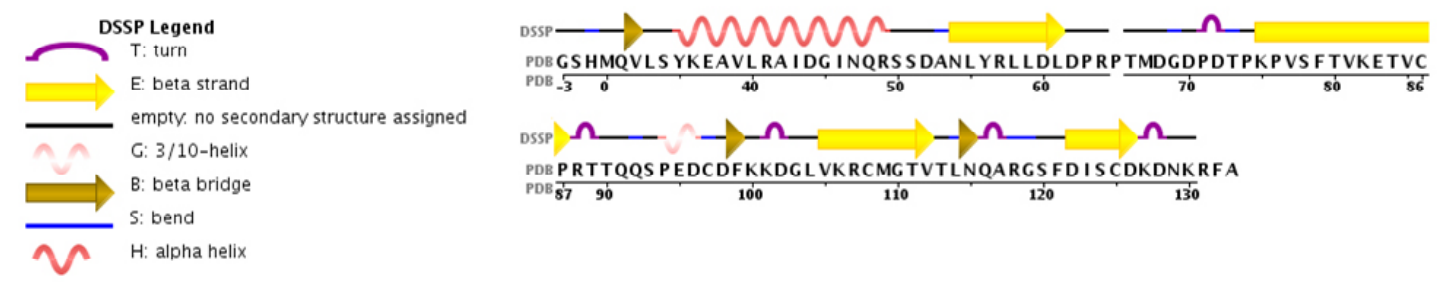

B
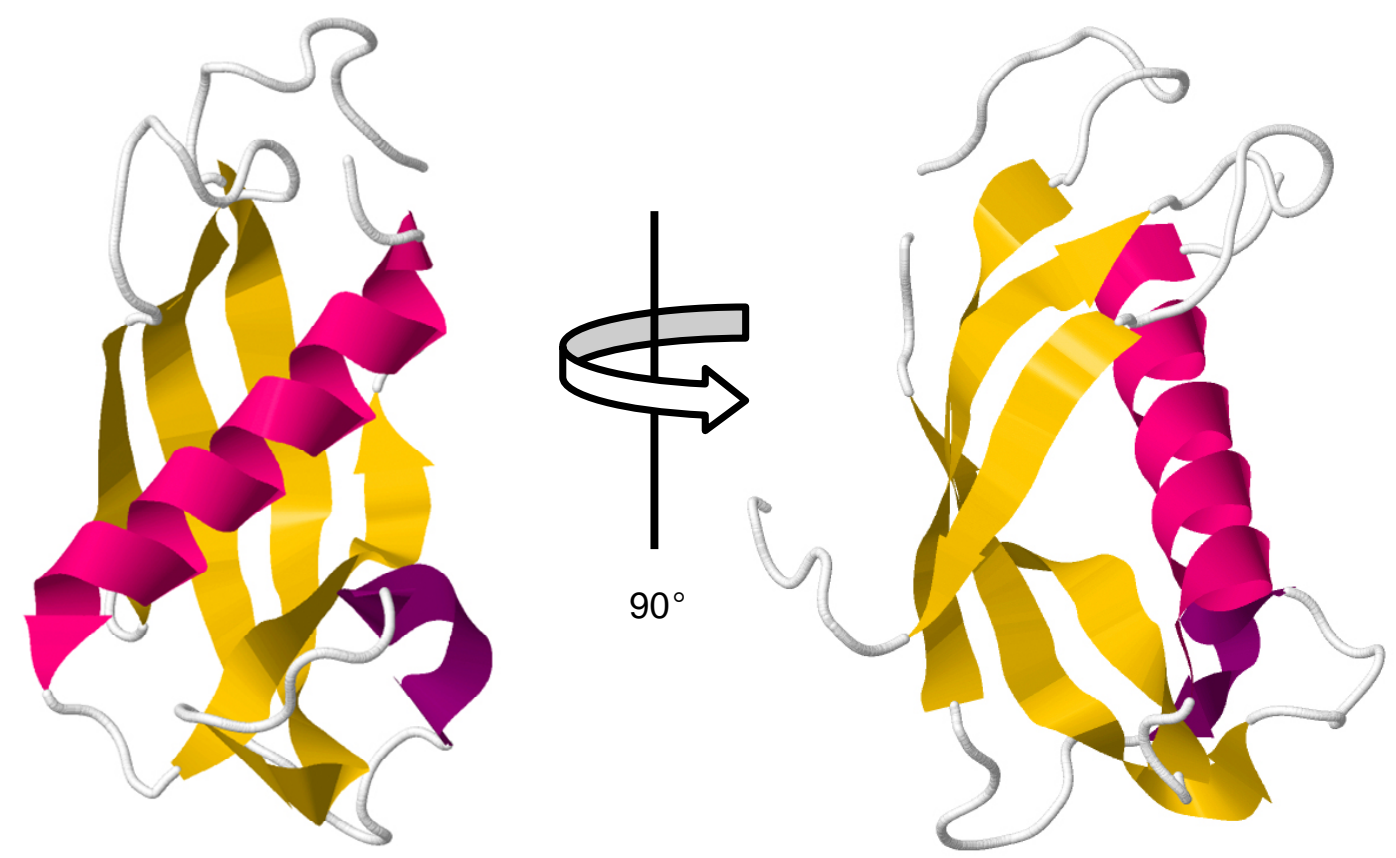

Figure 1.6 Topology and three-dimensional structure of the human CLD

(A) Topology diagram indicating the sequence of secondary structure elements in human CLD [100].

(B) Three-dimensional structure of the human CLD (Protein Data Bank ID: 4EYC [99]). 


\subsubsection{Localisation, processing and release}

Cathelicidins have been identified in myeloid cells of a number of species with significant variation in the number of homologues expressed [101]. Bovine cathelicidins are most abundant in neutrophil granules [86]. A 29 residue signal peptide at the $\mathrm{N}$-terminus directs cathelicidin accumulation into large granules during myeloid cell maturation.

Bovine MECs show negligible cathelicidin expression which is only slightly increased in response to pathogenic challenge (unpublished data, AgResearch, Food \& Bio-based Products Group, New Zealand). Many other bovine tissues do not express cathelicidins however cathelicidin-7 mRNA has been detected in spleen and testis [89]. In contrast, hCAP-18 is expressed in numerous tissues including many types of internal squamous epithelia, sweat and salivary glands, and testis [102]. Generally these tissues have mucosal surfaces that interface with luminal or external environments.

The signal sequence is cleaved by signal peptidase [103], releasing the unprocessed cathelicidin into the matrix of storage granules, or, into the extracellular environment in the case of epithelial cell production. Maturation of cathelicidin AMPs is governed by different neutral serine proteases in different species. Neutrophil elastase processes bovine and porcine cathelicidins [104,105] whereas cathepsin G processes hCAP-18 [106]. These proteases are stored in the primary granules of neutrophils or are excreted by epithelial cells, and come into physiological contact with cathelicidins after extracellular or phagosomal release.

The rapid influx of neutrophils during the innate immune response is usually paralleled by a large increase in the concentration of cathelicidins. For instance, in human urinary tract infection, cathelicidin concentration increases approximately eight-fold (from $0.3 \mathrm{ng} / \mathrm{ml}$ in healthy individuals to $2.4 \mathrm{ng} / \mathrm{ml}$ in infected individuals) [107]. During the onset of bovine mastitis the CLD concentration can increase greater than 300 -fold in clinical cases (from $300 \mathrm{ng} / \mathrm{ml}$ to $100 \mu \mathrm{g} / \mathrm{ml}$ ), and greater than 100 -fold in subclinical cases [108]. Hence, the cathelicidins have been proposed as a biomarker for the early detection of clinical mastitis and neutrophil influx [108]. 


\subsubsection{CLD activity}

\subsubsection{Structural functions}

The CLD has been described as a structural scaffold for the sequestration of the AMP domain prior to maturation by serine proteases [97]. Electrostatic interactions between the anionic surface of the CLD and the cationic AMP domain appear to inactivate the antimicrobial effects of the AMP domain [109]. Dissociation of the cleaved AMP domain from the CLD requires a $\mathrm{pH}$-dependent conformational change. NMR data has indicated a reversible change in conformation at acidic $\mathrm{pH}$ compared with neutral $\mathrm{pH}$ [97]. This implies that a proportion of AMPs released into phagosomes remain inactive until the phagosome is acidified by lysosomes. These electrostatic interactions must therefore be overcome in the extracellular inflammatory environment to activate the AMP. Exactly how this occurs is unclear.

The crystallisation study of porcine protegrin-3 showed a domain-swapped dimer could be produced in low $\mathrm{pH}$ and high ionic strength conditions [97]. The N-terminal helix and first $\beta$-sheet of each monomer is exchanged with the other, and the loop region between $\beta$-sheets one and two in each monomer forms an anti-parallel link between the monomers. Essential amino acid contacts are maintained in this configuration and the dimer backbone structure overlaps with the monomer backbone by $0.49 \AA$ RMSD. Physiologically, high molecular weight forms of hCAP-18 have been detected in human plasma [110], and two-dimensional electrophoresis analysis of mastitic milk indicates several high molecular weight forms of cathelicidin-1 exist in cattle [58]. This suggests oligomerisation or aggregation of the CLD may occur in vivo. Domain swapping is a feature of the cystatin superfamily which promotes the formation of amyloid-like fibrils which are implicated in the development of neurodegenerative diseases such as Alzheimer's, bovine spongiform encephalopathy (BSE) [111], and amyloid angiopathy [112]. A speculative function for the CLD may involve fibril or aggregate formation in the acidic environment of phagosomes which assist in permeabilisation of bacterial membranes. 


\subsubsection{Protease inhibition}

Cystatins are important immune system mediators which modulate cysteine protease activity and antigen presentation. The homology displayed between cystatins and the CLD suggested a mutual role in protease inhibition. The closest homologue, porcine cathelin, was shown to inhibit the plant cysteine protease papain and human cathepsin $\mathrm{L}$ with $\mathrm{K}_{\mathrm{i}}$ values of $0.1 \mathrm{nM}$ and $1 \mathrm{nM}$, respectively [113]. However later analysis of the cathelin isolation attributed this activity to a contaminating cysteine protease inhibitor [114].

Interestingly, significant variation in protease inhibitory activity has been observed for the CLD from different species. In assays of human cathepsin L activity, the human CLD was shown to moderately inhibit activity [115], while porcine protegrin-3 CLD significantly enhanced activity [116]. Further investigation suggested the second loop region between $\beta$-sheets three and four was responsible for activity, and these differed significantly between human CLD and protegrin-3 CLD. However, a more recent study using a similar inhibition assay showed human CLD had no protease inhibitory activity. Further analysis concluded this was due to the absence of specific residues in the first and second loop regions which are required for correct binding to proteases, as compared to established cysteine protease inhibitors [99].

Among the bovine cathelicidins, only cathelicidin- 2 has been investigated for inhibitory activity. It was found to inhibit cathepsin $\mathrm{L}$ with a $\mathrm{K}_{\mathrm{i}}$ of $60 \mathrm{nM}$ but was unable to inhibit human cathepsin B or papain [117]. Therefore cysteine protease inhibition appears to be a sporadic legacy from the divergence of the cathelicidin family and the cystatins, and indicates protease inhibition is not the driving force behind maintenance of the CLD structure.

\subsubsection{Chemotaxis}

The pro-form of bovine cathelicidin-3 was able to generate a chemotactic response in bovine monocytes [117]. This was attributable to the CLD as the AMP domain showed no chemotactic activity. The maximum chemotactic activity of the CLD was observed 
at $1 \mathrm{nM}$. The CLD of cathelicidin-2 was unable to evoke a similar response despite having $82 \%$ sequence identity [91].

\subsubsection{CLD activity summary}

The weak and sporadic activity exhibited by CLDs from different species indicates more of a structural role for the CLD. This is supported by the conservation of important secondary structure elements and the drifting variation in unordered regions between species. Whether the CLD is simply a structural scaffold for the activation of the AMP domain or whether higher molecular weight quaternary structure has significant activity remains to be determined. 


\subsubsection{AMP activity}

\subsubsection{Structure}

The length, charge, structure and sequence of cathelicidin AMPs are highly variable. Their length ranges between 12 and 100 amino acids with characteristically strong net positive charges at neutral $\mathrm{pH}$. Sub-classifications are based on structure and sequence, and are divided into $\alpha$-helical peptides, $\beta$-turn cysteine-bridged peptides and linear peptides. Linear peptides are further divided into proline and arginine-rich or tryptophan-rich peptides (Table 1.1). The properties of the seven bovine cathelicidin AMPs are given in Table 1.2.

Further processing of AMPs can occur either before or after cleavage from the CLD. C-terminal glycine residues can be converted to non-ionisable $\alpha$-amides $\left(-\mathrm{CONH}_{2}\right)$ by peptydylglycine $\alpha$-amidating monooxygenase. This occurs for the bovine peptides indolicidin, BMAP-28, BMAP-27 and BMAP-34, whereas the C-terminal proline is amidated for Bac5 [103]. This has also been reported for several porcine cathelicidin AMPs. The C-terminal amide group is thought to increase the net charge of the peptide thereby increasing electrostatic interaction with target membranes.

\subsubsection{Antimicrobial activity}

The cathelicidin AMPs were originally identified as antimicrobial secretions of neutrophils and particular tissues, and their antimicrobial effects have been examined on a large range of bacteria, yeast and viruses [67,84,102,118-122]. Because microorganisms rarely develop resistance to the toxic effects of these peptides, the main impetus of cathelicidin AMP research has been the development of peptide antibiotics $[123,124]$. More recently, antimicrobial peptide elicitors (APEs) have been proposed. These are chemical, biological or physical agents that rapidly upregulate endogenous antimicrobial peptides to combat infection [125]. The immunomodulatory effects of cathelicidin AMPs have a significant role in host defence against infection and have been less intensively studied. In a clinical setting the added effects of immunomodulatory activity may have great significance in the application of therapeutic AMPs and therefore warrant continued investigation. 
Table 1.1 Examples of cathelicidin AMP classification and diversity

\begin{tabular}{|c|c|c|c|c|c|c|}
\hline Structure & Origin & AMP & $\begin{array}{l}\text { Length } \\
\text { (aa) }\end{array}$ & $\begin{array}{c}\text { Net } \\
\text { charge }\end{array}$ & PTM & Reference \\
\hline \multirow[t]{19}{*}{ a-helical } & Feline (cat) & feCath & 37 & +3 & - & [126] \\
\hline & Galline (chicken) & Fowlicidin-1 & 26 & +8 & - & [119] \\
\hline & Galline (chicken) & Fowlicidin-2 & 32 & +10 & - & [119] \\
\hline & Galline (chicken) & Fowlicidin-3 & 29 & +7 & - & [119] \\
\hline & Bovine (cow) & BMAP-28 & 27 & +7 & $\alpha$-amidation & [127] \\
\hline & Bovine (cow) & BMAP-27 & 26 & +10 & $\alpha$-amidation & [127] \\
\hline & Bovine (cow) & BMAP-34 & 33 & +8 & $\alpha$-amidation & [89] \\
\hline & Canine (dog) & K9Cath & 38 & +6 & - & [128] \\
\hline & Equine (horse) & eCath-2 & 27 & +3 & - & [129] \\
\hline & Equine (horse) & eCath-3 & 40 & +7 & - & [129] \\
\hline & Human & LL-37 & 37 & +6 & - & [130] \\
\hline & Murine (mouse) & mCRAMP & 34 & +6 & - & [131] \\
\hline & Porcine (pig) & PMAP-23 & 23 & +6 & - & [132] \\
\hline & Porcine (pig) & PMAP-36 & 37 & +13 & - & [133] \\
\hline & Porcine (pig) & PMAP-37 & 37 & +3 & - & [134] \\
\hline & Lupine (rabbit) & CAP7 & 37 & +12 & - & [135] \\
\hline & Murine (rat) & rCRAMP & 43 & +8 & - & [136] \\
\hline & Rhesus monkey & RL-37 & 37 & +8 & - & [137] \\
\hline & Ovine (sheep) & SMAP-29 & 29 & +9 & - & [138] \\
\hline \multirow[t]{7}{*}{$\begin{array}{l}\beta \text {-sheet (disulfide } \\
\text { bond) }\end{array}$} & Bovine (cow) & Bac1 & 12 & +4 & - & [86] \\
\hline & Porcine (pig) & Protegrin-1 & 18 & +6 & $\alpha$-amidation & [139] \\
\hline & Porcine (pig) & Protegrin-2 & 16 & +5 & $\alpha$-amidation & [139] \\
\hline & Porcine (pig) & Protegrin-3 & 18 & +5 & $\alpha$-amidation & [139] \\
\hline & Porcine (pig) & Protegrin-4 & 18 & +4 & $\alpha$-amidation & [140] \\
\hline & Porcine (pig) & Protegrin-5 & 18 & +5 & $\alpha$-amidation & [91] \\
\hline & Ovine (sheep) & Bac1 & 12 & +4 & - & [141] \\
\hline \multirow[t]{6}{*}{ Linear (Pro/Arg-rich) } & Bovine (cow) & Bac5 & 43 & +9 & $\alpha$-amidation & [85] \\
\hline & Bovine (cow) & Bac7 & 60 & +17 & - & [85] \\
\hline & Porcine (pig) & PR-39 & 39 & +10 & $\alpha$-amidation & [142] \\
\hline & Porcine (pig) & Prophenin-2 & 79 & +6 & $\alpha$-amidation & [143] \\
\hline & Ovine (sheep) & Bac5 & 43 & +11 & $\alpha$-amidation & [122] \\
\hline & Ovine (sheep) & Bac7.5 & 60 & +20 & - & [141] \\
\hline Linear (Trp-rich) & Bovine (cow) & Indolicidin & 13 & +3 & a-amidation & [121] \\
\hline
\end{tabular}

Shaded rows designate examples from bovine. aa, amino acids. 
Table 1.2 Bovine cathelicidin AMP properties

\begin{tabular}{|lcccc|}
\hline Protein Name & AMP & Structure & Weight (kDa) & Feature \\
Cathelicidin-1 & Bac1 & B-sheet (disulfide bond) & 1.5 & Disulfide bond \\
Cathelicidin-2 & Bac5 & Linear/poly-proline helix & 5.1 & Proline and arginine rich \\
Cathelicidin-3 & Bac7 & Linear/poly-proline helix & 7.0 & Proline and arginine rich \\
Cathelicidin-4 & Indolicidin & Linear & 1.9 & Tryptophan rich \\
Cathelicidin-5 & BMAP-28 & $\alpha$-helical & 3.1 & Hydrophobic C-terminus \\
Cathelicidin-6 & BMAP-27 & $\alpha$-helical & 3.2 & Hydrophobic C-terminus \\
Cathelicidin-7 & BMAP-34 & $\alpha$-helical & 4.2 & Amphipathic
\end{tabular}

\subsubsection{Clinical applications of AMPs}

The relatively recent rise of antibiotic-resistant strains of bacteria has driven the search for alternative antibiotic therapies. AMPs have garnered much attention due to their rapid bactericidal activity, broad spectrum of activity and low incidence of resistance [144]. However, several drawbacks have hampered their release as approved therapies. Their limited stability, high cost and relatively unknown toxicology and pharmacology are often stumbling blocks to approval [144]. Despite this, many peptides are in various phases of clinical trials. For instance, Pexiganan is a topical cream containing magainin [145] that is being trialled in the treatment of diabetic foot ulcers [146]. Omiganan is also a topical treatment using a synthetic peptide derived from bovine indolicidin that is being trialled in the treatment of rosacea and catheter associated infections [147]. A final example is a synthetic peptide derived from LL-37, termed OP-145, that is being trialled in the treatment of chronic middle ear infection [148].

The increasing recognition of AMPs as multifunctional mediators of immune responses has led to alternative methods of boosting endogenous AMPs to fight infection [149]. An alternative to direct AMP application has been proposed in the form of APEs. Examples of APEs include ultraviolet light, vitamin D, nucleic acids, endogenous interleukins, transcription factors or hormones, and bacterial, plant or fungal lysates [125]. These elicitors could act by upregulating expression of endogenous antimicrobial peptides to combat infection. However, this approach is still at the conceptual stage and may be more effective in treating disease associated with abnormal expression of AMPs. 


\subsubsection{Immunomodulatory activity}

The nature of the antimicrobial effects of cathelicidins revealed an important interaction with negatively charged molecules on bacterial cell walls. The ability of AMPs to bind free LPS led to investigations of their ability to attenuate LPS-induced effects on immune cells. Additionally, the realisation that antimicrobial peptides might be suitable substitutes for conventional antibiotics led to investigations of cytotoxicity in mammalian cells. These initial avenues of research have broadened to include specific interactions with different cell types. Several examples of the immunomodulatory effects of AMPs on neutrophils are provided in the following sections. General immunomodulatory effects are summarised in Table 1.3 and Table 1.4.

The human cathelicidin AMP LL-37 induces several effects on human neutrophils via activation of fPRL1 and $\mathrm{P}_{2} \mathrm{X}_{7}$ receptors $[150,151]$. Activation induces intracellular calcium transients which mediate chemotaxis and ROS production. Other effects include induction of IL-8 expression [152] and induction of secondary necrosis in apoptotic neutrophils [153]. Multiple roles have also been identified for the porcine cathelicidin PR-39, including activation of neutrophil chemotaxis [154] and inhibition of respiratory burst [155]. Atlantic salmon cathelicidin AMPs can also induce expression of IL-8 in host neutrophils [156].

The effects of bovine cathelicidin AMPs on host defence have not been extensively studied. One minor study indicates that Bac5 and Bac7 are not able to induce bovine neutrophil or monocyte migration [117]. Another study found that BMAP-28 and BMAP-27 promote the secretion of TNF $\alpha$ from bovine MECs by an unknown mechanism [67]. In general, the remainder of the available research on the immunomodulatory properties of bovine cathelicidin AMPs has focused on their effects on non-bovine cells (Table 1.3), as a precursor to potential therapeutic antibiotic applications. This has left a large gap in the collective understanding of the roles that the different bovine cathelicidins carry out in host defence. 


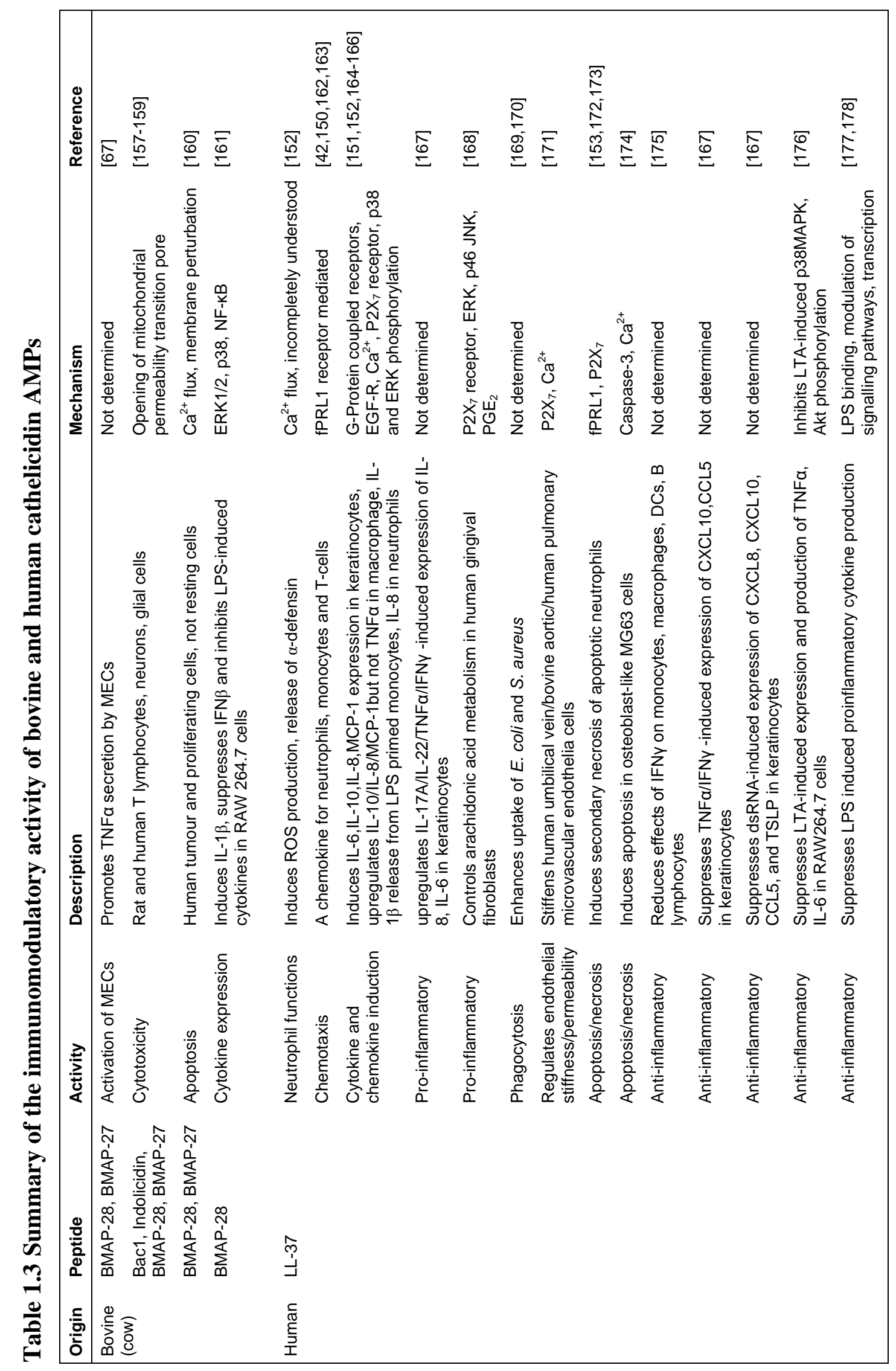




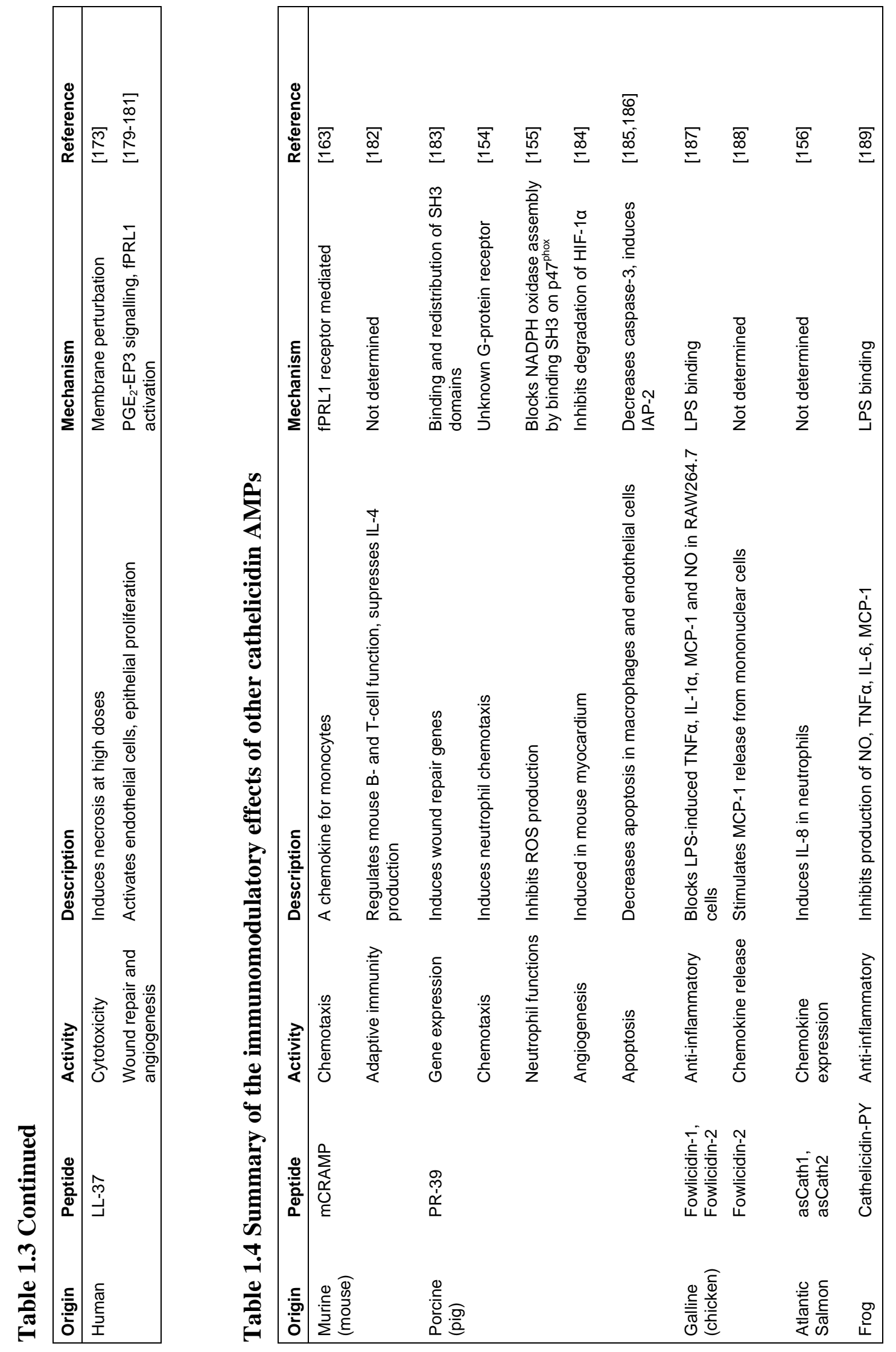




\subsection{Aims}

The broad aim of this thesis was to investigate the role of bovine cathelicidins in host defence, in particular the effect of bovine cathelicidins on bovine neutrophil functions.

The specific aims of this thesis were:

1. To investigate the role of the bovine CLD in innate immune defence.

2. To profile the immunomodulatory effects of the different bovine AMP domains on neutrophil function.

3. To investigate the mechanism by which active bovine AMPs exert their immunomodulatory effects on neutrophils. 
Chapter 2: Materials and Methods 


\subsection{Peptide synthesis and preparation}

All peptides were synthesised by AusPep Proprietary Limited (Tullamarine, Australia) (Table 2.1). Mass and purity were determined by mass spectrometry (MS) and high performance liquid chromatography, respectively. The peptides were weighed and reconstituted to approximately $1 \mathrm{mM}$ in endotoxin-free water. Serial dilutions of the $1 \mathrm{mM}$ stock were used to measure the actual concentration of peptide. For Bac1 and linear Bac1 a bicinchoninic acid (BCA) assay (section 2.3.3.3) was used to measure the actual concentrations of peptide serial dilutions. For all other peptides a NanoDrop ND1000 Spectrophotometer (Thermo Fisher Scientific New Zealand Ltd., North Shore City, New Zealand) was used to measure the concentration of serial dilutions based on extinction coefficients at $280 \mathrm{~nm}$ for peptides containing tryptophan or tyrosine (Bac5, indolicidin, BMAP-28, RITK), or $257 \mathrm{~nm}$ for peptides containing phenylalanine (BMAP-34 , LL-37) [190]. The $1 \mathrm{mM}$ stock was then adjusted to approximately $500 \mu \mathrm{M}$ and retested.

For fluorescein isothiocyanate (FITC)-conjugated Bac5 (Bac5-FITC) (excitation/emission $495 \mathrm{~nm} / 519 \mathrm{~nm}$ ), the peptide was reconstituted in dimethyl sulfoxide (Sigma-Aldrich, St Lewis, MO, USA) to approximately $5 \mathrm{mM}$. Serial dilutions of the $5 \mathrm{mM}$ stock were measured for absorbance at $280 \mathrm{~nm}$ and $495 \mathrm{~nm}$ using a NanoDrop ND-1000 Spectrophotometer. Peptide concentration was determined using the equation given below [191], which takes into account FITC absorbance. The $5 \mathrm{mM}$ stock was adjusted accordingly and retested.

$$
\operatorname{Bac5-FITC}(\mathrm{mg} / \mathrm{ml})=\frac{A_{280}-\left(0.35 \times A_{495}\right)}{A_{280}(\text { at } 1.0 \mathrm{mg} / \mathrm{ml}, \mathrm{pH} \mathrm{7.0})}
$$


Table 2.1 Synthetic peptide sequences

\begin{tabular}{|c|c|c|c|c|}
\hline Origin & Peptide & Sequence & $\begin{array}{l}\text { Weight } \\
\text { (Da) }\end{array}$ & $\begin{array}{l}\text { Extinction } \\
\text { coefficient } \\
\left(\mathrm{M}^{-1} \mathrm{~cm}^{-1}\right)\end{array}$ \\
\hline $\begin{array}{l}\text { Bovine } \\
\text { Cathelicidin-1 }\end{array}$ & Bac1 & RLCRIVVIRVCR & 1484 & $\begin{array}{c}125 \\
(280 \mathrm{~nm})\end{array}$ \\
\hline $\begin{array}{l}\text { Bovine } \\
\text { Cathelicidin-1 }\end{array}$ & Linear Bac1 & RLCRIVVIRVCR & 1486 & 0 \\
\hline $\begin{array}{l}\text { Bovine } \\
\text { Cathelicidin-2 }\end{array}$ & Bac5 & $\begin{array}{l}\text { RFRPPIRRPPIRPPFYPPFRPPIRPPIFPPIRPPF } \\
\text { RPPLGPFP-NH2 }\end{array}$ & 5148 & $\begin{array}{c}1490 \\
(280 \mathrm{~nm})\end{array}$ \\
\hline $\begin{array}{l}\text { Bovine } \\
\text { Cathelicidin-2 }\end{array}$ & Bac5-FITC & $\begin{array}{l}\text { FITC-RFRPPIRRPPIRPPFYPPFRPPIRPPIFPPIR } \\
\text { PPFRPPLGPFP-NH2 }\end{array}$ & 5608 & $\begin{array}{c}1490 \\
(280 \mathrm{~nm})\end{array}$ \\
\hline $\begin{array}{l}\text { Bovine } \\
\text { Cathelicidin-4 }\end{array}$ & Indolicidin & ILPWKWPWWPWRR-NH2 & 1907 & $\begin{array}{c}27500 \\
(280 \mathrm{~nm})\end{array}$ \\
\hline $\begin{array}{l}\text { Bovine } \\
\text { Cathelicidin-5 }\end{array}$ & BMAP-28 & GGLRSLGRKILRAWKKYGPIIVPIIRI-NH2 & 3075 & $\begin{array}{c}6990 \\
(280 \mathrm{~nm})\end{array}$ \\
\hline $\begin{array}{l}\text { Bovine } \\
\text { Cathelicidin-7 }\end{array}$ & BMAP-34 & GLFRRLRDSIRRGQQKILEKARRIGERIKDIFR-NH2 & 4082 & $\begin{array}{c}390 \\
(257 \mathrm{~nm})\end{array}$ \\
\hline $\begin{array}{l}\text { Human } \\
\text { hCAP-18 }\end{array}$ & LL-37 & $\begin{array}{l}\text { LLGDFFRKSKEKIGKEFKRIVQRIKDFLRNLVPR } \\
\text { TES-OH }\end{array}$ & 4493 & $\begin{array}{c}780 \\
(257 \mathrm{~nm})\end{array}$ \\
\hline $\begin{array}{l}\text { Bovine } \\
\text { Cathelicidin-1 }\end{array}$ & RITK & $\begin{array}{l}\text { RITKQPWAPPQAA } \\
\text { (putative } 2^{\text {nd }} \text { cathelicidin- } 1 \text { peptide) }\end{array}$ & 1464 & $\begin{array}{c}5500 \\
(280 \mathrm{~nm})\end{array}$ \\
\hline
\end{tabular}




\subsection{In-house antibody preparation}

\subsubsection{Cathelicidin-specific A14 and A32 antibodies}

The A14 and A32 antibodies were prepared by Grant Smolenski (Food \& Bio-based Products Group, AgResearch, New Zealand). Synthetic peptides (AusPep Proprietary Limited) were coupled to keyhole limpet haemocyanin (KLH) (Sigma-Aldrich). The peptide sequence for the A14 antibody was CNEQSSEPNIYRLLELDQ, which corresponds to a sequence which is conserved across all bovine CLDs. Hence the A14 antibody binds to all bovine CLDs. The peptide sequence for the A32 antibody was CIRITKQPWAPPQAA, which corresponds to a specific sequence of bovine cathelicidin-1 CLD. Hence the A32 antibody only binds to the CLD of cathelicidin- 1 . Inoculation of rabbits and serum collection was carried out under animal ethics approval by Ric Broadhurst (Small Animal Colony, AgResearch, Ruakura, New Zealand). Purification of IgG antibody was carried out using a Protein A agarose column (SigmaAldrich) and specificity for the target protein was verified by western blot.

\subsubsection{Transcobalamin II antibody}

Anti-transcobalamin II (TCII) antibody was prepared by Tanu Gupta (Food \& Biobased Products Group, AgResearch, New Zealand). A synthetic peptide (AusPep Proprietary Limited) with the sequence SQLKRFLEDEKRAIGH, corresponding to a sequence within bovine transcobalamin II, was coupled to KLH. Inoculation of rabbits and serum collection was carried out under animal ethics approval by Ric Broadhurst. Purification of $\mathrm{IgG}$ antibody was carried out using a Protein A agarose column and specificity for the target protein was verified by western blot. 


\subsection{Molecular biology techniques}

\subsubsection{Protein expression}

\subsubsection{Open reading frame selection, PCR and cloning}

Cathelicidin-1 open reading frame PCR, PCR site-directed mutagenesis and cloning into Novagen pET-45b(+) vector (EMD Millipore, MA, USA) was previously carried out by Brendan Haigh (Food \& Bio-based Products Group, AgResearch, New Zealand). This generated plasmid vectors encoding the CLD of cathelicidin-1 (pET_Cath1_15/13new5; Q30 to A143, P22226 [91]) to express recombinant bovine CLD (rbCLD), and a full length cathelicidin-1 protein containing an A143 to F143 mutation (pET_Cath1_NER2; Q30 to R155, P22226 [91]) to express recombinant bovine Cath1 (rbCath1). The mutation in rbCath1 was designed to prevent proteolytic cleavage of Bac1 AMP from the CLD.

\subsubsection{Transformation}

Novagen E. coli Origami (EMD Millipore) were transformed with $1 \mu 1$ of each plasmid by incubating on ice for 5 minutes, incubating at $42{ }^{\circ} \mathrm{C}$ for 30 seconds and returning to ice for 2 minutes. Control transformations contained no plasmid. Cells were supplemented with $250 \mu \mathrm{l}$ super optimal broth with catabolite repression media (SOC media, Life Technologies Inc., NY, USA) and incubated for 60 minutes at $37^{\circ} \mathrm{C}$. The cells were centrifuged and $50 \mu \mathrm{l}$ suspensions were plated on Luria broth agar plates containing kanamycin, tetracycline and ampicillin antibiotics (LB/kan/tet/amp agar) (Appendix 2) and incubated overnight at $37^{\circ} \mathrm{C}$.

\subsubsection{Small scale expression test}

Colonies from each transformation were used to inoculate $2 \mathrm{ml} \mathrm{LB} / \mathrm{kan} / \mathrm{tet} / \mathrm{amp}$ (Appendix 2) which were incubated overnight with shaking at $37^{\circ} \mathrm{C}$. Each overnight culture was added to $50 \mathrm{ml} \mathrm{LB} / \mathrm{kan} / \mathrm{tet} / \mathrm{amp}$ in $250 \mathrm{ml}$ flasks and incubated with shaking at $37^{\circ} \mathrm{C}$. The optical density at $600 \mathrm{~nm}\left(\mathrm{OD}_{600}\right)$ was measured using an Eppendorf Biophotometer (Eppendorf, Hamburg, Germany). Incubation was stopped when the $\mathrm{OD}_{600}$ reached 0.7 AU. A $2 \mathrm{ml}$ sample of uninduced culture was removed for sodium dodecyl sulfate-polyacrylamide gel electrophoresis (SDS-PAGE) analysis (section 2.3.3.4). The remaining culture was induced with $62.5 \mu 10.8 \mathrm{M}$ isopropyl $\beta$-D- 
1-thiogalactopyranoside (IPTG) (Life Technologies Inc.) and incubated for 90 minutes or 180 minutes at $37^{\circ} \mathrm{C}$. A $2 \mathrm{ml}$ sample of induced culture was removed for SDS-PAGE analysis.

The pellets of samples for SDS-PAGE analysis were weighed and resuspended in $5 \times$ Tris-ethylenediaminetetraacetic acid (EDTA) (5×TE) (Appendix 2) containing complete EDTA-free protease inhibitors (Roche Diagnostics N.Z., Ltd., Auckland, New Zealand). The cells were sonicated (Vibra Cell sonicator, Sonics \& Materials Inc., CT, USA) on ice five times with 10 second bursts. The lysed cells were centrifuged at $10,000 \times \mathrm{g}$ for 10 minutes at $4{ }^{\circ} \mathrm{C}$. The supernatant was removed (soluble protein fraction) and $10 \mu \mathrm{l}$ was prepared for SDS-PAGE. The pellet was resuspended in $5 \times \mathrm{TE}$ at $10 \mathrm{ml} / \mathrm{g}$ of pellet (insoluble protein fraction) and $10 \mu \mathrm{l}$ was prepared for SDS-PAGE.

\subsubsection{Glycerol stock}

Two aliquots of $0.5 \mathrm{ml}$ uninduced culture were added to $0.5 \mathrm{ml} 80 \%$ (v/v) glycerol (Thermo Fisher Scientific New Zealand Ltd.), rotated for 30 minutes at room temperature and stored at $-80{ }^{\circ} \mathrm{C}$.

\subsubsection{Large scale expression of rbCLD}

Two $5 \mathrm{ml}$ overnight cultures inoculated from glycerol stock were added to two $2 \mathrm{~L}$ flasks containing $500 \mathrm{ml} \mathrm{LB} / \mathrm{kan} / \mathrm{tet} / \mathrm{amp}$, and incubated with shaking at $37{ }^{\circ} \mathrm{C}$ until the $\mathrm{OD}_{600}$ reached 0.7 AU. Samples were taken as described in section 2.3.1.3 and analysed by SDS-PAGE. The cultures were induced with $625 \mu 10.8 \mathrm{M}$ IPTG and incubated for 3 hours at $37{ }^{\circ} \mathrm{C}$. The cultures were centrifuged at $6000 \mathrm{rpm}$ for 15 minutes at $4{ }^{\circ} \mathrm{C}$. The supernatant was discarded and the pellets transferred into two $50 \mathrm{ml}$ tubes and frozen at $-80{ }^{\circ} \mathrm{C}$ until required.

\subsubsection{Large scale expression of rbCath1}

Two $3 \mathrm{ml}$ overnight cultures inoculated from glycerol stock were added to two $1 \mathrm{~L}$ flasks containing $200 \mathrm{ml} \mathrm{LB} / \mathrm{kan} / \mathrm{tet} / \mathrm{amp}$ and incubated with shaking at $37{ }^{\circ} \mathrm{C}$ until the $\mathrm{OD}_{600}$ reached 0.7 AU. Samples were taken as described in section 2.3.1.3 and analysed by SDS-PAGE. The cultures were induced with $250 \mu 10.8 \mathrm{M}$ IPTG and incubated for 
3 hours at $37^{\circ} \mathrm{C}$. The cultures were centrifuged at $6000 \mathrm{rpm}$ for 15 minutes at $4{ }^{\circ} \mathrm{C}$. The supernatant was discarded and the pellets transferred into two $50 \mathrm{ml}$ tubes and frozen at $-80^{\circ} \mathrm{C}$ until required.

\subsubsection{Protein purification}

\subsubsection{Column preparation and storage}

A $1 \mathrm{ml}$ HisTrap Fast Flow immobilised metal ion affinity chromatography (IMAC) column (GE Healthcare Ltd., Auckland, New Zealand) was prepared by flushing with 3 column volumes (CV) of column stripping solution (Appendix 2) followed by $10 \mathrm{CV}$ of milli-Q water (MQ. $\left.\mathrm{H}_{2} \mathrm{O}\right)$. The column was loaded with $\mathrm{Ni}^{2+}$ by passing $1 \mathrm{CV}$ of nickel loading solution (Appendix 2) through the column, followed by $10 \mathrm{CV}$ of MQ. $\mathrm{H}_{2} \mathrm{O}$ to remove excess $\mathrm{Ni}^{2+}$. The column was then equilibrated with $6 \mathrm{CV}$ of appropriate buffer. After use the column was cleaned by passing $10 \mathrm{CV}$ of lysis buffer A (Appendix 2) through the column, followed by $10 \mathrm{CV} \mathrm{MQ} \cdot \mathrm{H}_{2} \mathrm{O}$, then $3 \mathrm{CV}$ of column storage solution (Appendix 2).

\subsubsection{IMAC purification of rbCLD}

The pellets from section 2.3.1.5 were resuspended in lysis buffer A containing $1 \mathrm{mg} / \mathrm{ml}$ lysozyme (Sigma-Aldrich) and complete EDTA-free protease inhibitors, and incubated on ice for 30 minutes. The cells were sonicated on ice five times with 30 second bursts. The lysed cells were centrifuged at $10,000 \times \mathrm{g}$ for 10 minutes at $4{ }^{\circ} \mathrm{C}$. The pellet was retained for SDS-PAGE analysis. The supernatant was loaded onto a $1 \mathrm{ml}$ HisTrap FF column, pre-equilibrated with lysis buffer A, through a $0.45 \mu \mathrm{m}$ filter (Sartorius, Göttingen, Germany). The column was flushed with $10 \mathrm{CV}$ wash buffer A (Appendix 2). The flow through was collected in $15 \mathrm{ml}$ tubes. A second wash of $10 \mathrm{CV}$ was performed and the flow through was collected. Six CV of elution buffer A (Appendix 2) was then passed through the column and the flow through was collected. The elution procedure was repeated and the flow through was collected in a separate tube. Aliquots of $20 \mu \mathrm{l}$ were taken from each flow through collection for SDS-PAGE analysis. Imidazole was removed from the elution fractions by buffer exchange with dialysis buffer (Appendix 2) using a Vivaspin 6 centrifugal concentrator with $10 \mathrm{kDa}$ molecular weight cut-off (GE Healthcare Ltd.). 


\subsubsection{IMAC purification and on-column refolding of rbCath1}

The pellets from section 2.3.1.6 were prepared as described in section 2.3.2.2 using lysis buffer B (Appendix 2). The supernatant was loaded onto a $1 \mathrm{ml}$ HisTrap FF column, pre-equilibrated with lysis buffer $B$, through a $0.45 \mu \mathrm{m}$ filter. The column was flushed with $6 \mathrm{CV}$ wash buffer B (Appendix 2) and the flow through was collected. The column was then connected to an AKTA Explorer system (GE Healthcare Ltd.) equilibrated with wash buffer B. The system was then programmed (UNICORN software, GE Healthcare Ltd.) to run a gradient of $0 \%$ to $100 \%$ refold buffer B (Appendix 2) over 4 hours. The flow through of the gradient was collected. The column was then removed from the system and refolded protein eluted with $10 \mathrm{CV}$ elution buffer B (Appendix 2). Aliquots of $20 \mu \mathrm{l}$ were taken from each flow through for SDS-PAGE analysis. Trichloroacetic acid (TCA) precipitation (section 2.3.3.1) was used to remove guanidine hydrochloride from samples prior to SDS-PAGE analysis. To remove imidazole and glycerol from the elution fraction, refolded rbCath1 was dialysed against dialysis buffer (Appendix 2) using Spectra/Por 6 dialysis membrane (3,500 Da, Spectrum Laboratories Inc., CA, USA).

\subsubsection{Concentration of purified protein}

Both recombinant proteins were concentrated using a Vivaspin 500 centrifugal concentrator with $10 \mathrm{kDa}$ molecular weight cut-off (GE Healthcare Ltd.). Correct expression of rbCLD and rbCath1 was determined by western blot analysis (section 2.3.3.5). Primary antibody incubation was for 2 hours at room temperature using rabbit A32 antibody (section 2.2.1) diluted 1:10,000 in antibody diluent A (Appendix 2). Secondary antibody incubation was for 1 hour at room temperature using peroxidase-conjugated goat anti-rabbit antibody (A6154, Sigma-Aldrich) diluted 1:10,000 in antibody diluent A. MS was also used to confirm rbCLD expression (section 2.8.1).

\subsubsection{His·Tag removal}

A Novagen recombinant enterokinase (rEK) cleavage-capture kit (EMD Millipore) was used to remove the His-Tag from recombinant protein according to the manufacturer's instructions. Recombinant protein was dialysed with $1 \times$ rEK cleavage capture buffer 
(Appendix 2) to prevent inhibition of rEK by elution buffer components. The system was then optimised for incubation time and enzyme to target protein ratio. Free His. Tag was removed from solution by passage through an IMAC column. The flow through containing the target protein was concentrated by centrifugation with buffer exchange as described in section 2.3.2.4.

\subsubsection{Protein detection}

\subsubsection{TCA precipitation}

To precipitate protein from solution, one volume of 90\% TCA (Riedel-de-Haën, Seelze, Germany) was added to four volumes of protein solution and incubated for 10 minutes on ice. Precipitated protein was centrifuged at $13,000 \mathrm{rpm}$ for 5 minutes at $4{ }^{\circ} \mathrm{C}$. The supernatant was discarded and the pellet was washed with $200 \mu \mathrm{l}$ cold acetone (Thermo Fisher Scientific New Zealand Ltd.). The wash procedure was repeated and the pellet dried by heating at $95^{\circ} \mathrm{C}$ for 10 minutes. The dried pellet was then prepared for SDSPAGE analysis (section 2.3.3.4).

\subsubsection{Bio-Rad Protein assay}

The Bio-Rad Protein assay (Bio-Rad, Richmond, CA, USA) is based on the colour change of Coomassie Brilliant Blue (CBB) G-250 when protein binding occurs. The following protocol is based on the manufacturer's instructions. Bovine serum albumin (BSA) (Sigma-Aldrich) standards were made by diluting $2 \mathrm{mg} / \mathrm{ml} \mathrm{BSA} \mathrm{stock} \mathrm{in} \mathrm{the}$ unknown sample buffer to create six standards from $30 \mu \mathrm{g} / \mathrm{ml}$ to $180 \mu \mathrm{g} / \mathrm{ml}$. Several dilutions of the unknown samples were also prepared in the same buffer. A Bio-Rad Protein assay working solution was made by diluting the assay dye concentrate 1:5 in MQ. $\mathrm{H}_{2} \mathrm{O}$. In $1.5 \mathrm{ml}$ tubes, $900 \mu \mathrm{l}$ of assay working solution was added to $100 \mu \mathrm{l}$ of standards and $100 \mu \mathrm{l}$ of unknown samples and dilutions. Each solution was transferred to an Eppendorf UVette with $1 \mathrm{~cm}$ light path (Eppendorf, Hamburg, Germany) and the absorbance was measured at $595 \mathrm{~nm}$ in a Shimadzu UV-160A spectrophotometer (Shimadzu, Kyoto, Japan). 


\subsubsection{BCA assay}

The BCA assay is based on the formation of $\mathrm{Cu}^{2+}$-protein complexes under alkaline conditions, followed by reduction of $\mathrm{Cu}^{2+}$ to $\mathrm{Cu}^{1+}$. The following protocol is from the technical bulletin of the BCA protein assay kit (BCA1, Sigma-Aldrich) based on the method of Smith, et al. [192]. BSA standards were made by diluting a $2 \mathrm{mg} / \mathrm{ml}$ BSA stock in the unknown sample buffer to create eight standards from $25 \mu \mathrm{g} / \mathrm{ml}$ to $2 \mathrm{mg} / \mathrm{ml}$. Several dilutions of the unknown samples were also prepared in the same buffer. The BCA working reagent was made by diluting $4 \%(\mathrm{w} / \mathrm{v})$ copper (II) sulfate pentahydrate solution 1:8 in bicinchoninic acid solution. In 96-well flat bottom plates (Nunc, Denmark), $200 \mu \mathrm{l}$ of BCA working reagent was added to $25 \mu \mathrm{l}$ of standards in duplicate and $25 \mu \mathrm{l}$ of unknown samples and dilutions in duplicate. The plate was incubated for 30 minutes at $37{ }^{\circ} \mathrm{C}$ and the absorbance measured at $562 \mathrm{~nm}$ with a Synergy 2 multimode microplate reader (BioTek Instruments Inc., VT, USA). Data were analysed with Gen5 software (BioTek Instruments Inc.).

\subsubsection{SDS-PAGE analysis}

\subsection{Preparation of samples}

Samples for SDS-PAGE analysis were prepared by adding appropriate volumes of $3 \times$ sample loading buffer (SLB) (Appendix 2) and $\mathrm{MQ} \cdot \mathrm{H}_{2} \mathrm{O}$ to protein solutions or precipitates. All samples were boiled for 10 minutes and allowed to cool before loading. Typical loading volumes were between $5 \mu \mathrm{l}$ and $10 \mu \mathrm{l}$ for mini polyacrylamide gels or between $10 \mu \mathrm{l}$ and $20 \mu \mathrm{l}$ for precast polyacrylamide gels. A range of protein molecular weight standards were used for each electrophoresis run depending on the procedures subsequent to electrophoresis. These standards were SeeBlue Plus2 Pre-Stained Standard (Life Technologies Inc.), Precision Plus Protein WesternC Standards (BioRad), and Natural Unstained Broad Range SDS-PAGE Standards (Bio-Rad).

\subsection{In-house mini polyacrylamide gels}

Mini gels were made using a Mini-Protean 3 Cell (Bio-Rad) according to the instructions provided. The compositions of mini gels are provided in Table 2.2. The gels were run in a mini tank using $1 \times$ running buffer (Appendix 2) at a constant voltage of $200 \mathrm{~V}$ for 70 minutes. 
Table 2.2 Mini polyacrylamide gel composition

\begin{tabular}{|cccc|}
\hline Reagent & Stacking gel & $10 \%$ Resolving gel & $14 \%$ Resolving gel \\
\hline $30 \%\left(\right.$ w/v) Acrylamide ${ }^{\mathrm{a}}$ & $0.66 \mathrm{ml}$ & $3.33 \mathrm{ml}$ & $4.67 \mathrm{ml}$ \\
$4 \times$ stacking buffer $^{\mathrm{b}}$ & $1.23 \mathrm{ml}$ & - & - \\
$4 \times$ resolving buffer & - & $2.5 \mathrm{ml}$ & $2.5 \mathrm{ml}$ \\
MQ. $_{2} \mathrm{O}$ & $3.08 \mathrm{ml}$ & $4.17 \mathrm{ml}$ & $2.33 \mathrm{ml}$ \\
$10 \%(\mathrm{w} / \mathrm{v})$ APS $^{\mathrm{a}}$ & $25 \mu \mathrm{l}$ & $30 \mu \mathrm{l}$ & $30 \mu \mathrm{l}$ \\
TEMED $^{\mathrm{a}}$ & $10 \mu \mathrm{l}$ & $10 \mu \mathrm{l}$ & $10 \mu \mathrm{l}$ \\
\hline
\end{tabular}

${ }^{\mathrm{a}}$ from Bio-Rad; ${ }^{\mathrm{b}}$ see Appendix 2.

\subsection{Precast polyacrylamide gels}

Criterion XT 12\% Bis-Tris precast gels (Bio-Rad) were used prior to western blot or far-western blot procedures. The gels were run in a Criterion Cell buffer tank (Bio-Rad) using 3-(N-morpholino)propanesulfonic acid (MOPS) buffer (Life Technologies Inc.) at a constant voltage of $160 \mathrm{~V}$ for 70 minutes.

\subsection{CBB R-250 stain}

Polyacrlyamide gels were fixed in fixative/destain solution (Appendix 2) for 30 minutes at room temperature, briefly rinsed in $\mathrm{MQ} \cdot \mathrm{H}_{2} \mathrm{O}$ and stained with $\mathrm{CBB}$ R-250 stain solution (Appendix 2) for 60 minutes at room temperature. The gels were destained with fix/destain solution at room temperature until the background was clear and analysed as described in section 2.3.3.9.

\subsubsection{Western blot procedure}

An electrotransfer cassette was prepared under N-cyclohexyl-3-aminopropanesulfonic acid (CAPS) transfer buffer (Appendix 2) by layering blotting pad, two sheets of presoaked Whatman 3MM chromatography paper (GE Healthcare Ltd.), electrophoresed polyacrylamide gel, pre-soaked BioTrace NT nitrocellulose membrane (Pall Corporation, FL, USA), two sheets of pre-soaked 3MM and the top blotting pad. The stack was rolled to remove air bubbles and the cassette transferred to a blotting tank containing CAPS transfer buffer. The gel was transferred for 2 hours at $50 \mathrm{~V}$.

After transfer the cassette was disassembled and the membrane was stained for 5 minutes in Ponceau S stain (Appendix 2) and analysed as described in section 2.3.3.9. 
The membrane was then transferred to blocking solution A (Appendix 2) for 16 hours at $4{ }^{\circ} \mathrm{C}$. After blocking, and after primary and secondary antibody incubations, the membrane of each western blot was washed three times for 5 minutes in wash solution A (Appendix 2). Primary and secondary antibody incubation conditions were optimised for the detection of the specific protein. These conditions are stated in the sections where the western blot procedure was applied. Bound antibody was visualised using enzyme-linked chemiluminescence (ECL) (section 2.3.3.7).

\subsubsection{Dot blot procedure}

Dot blots were carried out using a Bio-Dot SF blotting apparatus (Bio-Rad). Samples were blotted onto BioTrace NT nitrocellulose membrane according to the instructions provided. The apparatus was disassembled and the membrane was stained for 5 minutes in Ponceau S stain and analysed as described in section 2.3.3.9. The membrane was then transferred to blocking solution A for 16 hours at $4{ }^{\circ} \mathrm{C}$. After blocking, and after primary and secondary antibody incubations, the membrane of each dot blot was washed three times for 5 minutes in wash solution A (Appendix 2). Primary and secondary antibody incubation conditions were optimised for the detection of the specific protein. These conditions are stated in the section where the dot blot procedure was applied. Bound antibody was visualised using ECL.

\subsubsection{ECL detection}

For ECL detection of bound antibody, an ECL solution was made containing $220 \mu \mathrm{g} / \mathrm{ml}$ luminol (Sigma-Aldrich), $11.2 \mu \mathrm{g} / \mathrm{ml} p$-coumaric acid (Sigma-Aldrich), and 0.01\% (v/v) $\mathrm{H}_{2} \mathrm{O}_{2}$ (Thermo Fisher Scientific New Zealand Ltd.) in $100 \mathrm{mM}$ Tris (pH 8.6) (Fisher BioReagents, NJ, USA). Following the final wash after secondary antibody incubation, the membrane was incubated in the ECL solution for 2 minutes. Photographic film (Kodak, Radiographic Supplies Ltd., New Zealand) was then exposed to the membrane in a light-free cassette (Kodak, Radiographic Supplies Ltd.). The developed photographic film was analysed as described in section 2.3.3.9. 


\subsubsection{Far-western blot procedure}

After SDS-PAGE, a transfer cassette was assembled with PVDF membrane (Bio-Rad) as described in section 2.3.3.5. After transfer the cassette was disassembled and the membrane was stained for 5 minutes in Ponceau $\mathrm{S}$ stain and analysed as described in section 2.3.3.9. The membrane was then transferred to blocking solution $B$ (Appendix 2) for 16 hours at $4{ }^{\circ} \mathrm{C}$. After blocking, and after probe incubations, the membrane of each far-western blot was washed three times for 5 minutes in wash solution B (Appendix 2). Probe incubation conditions were optimised to improve signal to noise ratio. These conditions are stated in the sections where the far-western blot procedure was applied. Bound probe was visualised and analysed as described in section 2.3.3.9.

\subsubsection{Semi-quantitative analysis of polyacrylamide gels and membranes}

Stained polyacrylamide gels, membranes stained with Ponceau S, and developed photographic film were scanned using a GS800 Calibrated Densitometer (Bio-Rad) and analysed using Quantity One 1-D analysis software (v4.5.2, Bio-Rad).

Unstained polyacrylamide gels containing Bac5-FITC, and far-western blot membranes probed with Bac5-FITC were scanned using a Molecular Imager FX (Bio-Rad) with $488 \mathrm{~nm}$ excitation laser. Images were analysed using Quantity One 1-D analysis software. 


\subsection{LPS-binding assays}

\subsubsection{Recombinant cathelicidin LPS-binding assay}

Purified rbCLD, rbCath1, native Cath1, LBP and casein were diluted to $10 \mu \mathrm{g} / \mathrm{ml}$ in phosphate buffered saline (PBS) (Appendix 2) and plated in $50 \mu$ triplicates in flatbottomed 96-well microplates (MaxiSorp, Nunc). The microplates were incubated for 16 hours at room temperature then washed three times with wash solution A (Appendix 2) between each step. Plates were blocked with $1 \%(\mathrm{w} / \mathrm{v})$ casein in PBS for 2 hours followed by $1 \mu \mathrm{g} / \mathrm{ml}$ biotin-conjugated LPS (Sigma-Aldrich) for 1 hour. Bound LPS was visualised with streptavidin-conjugated horseradish peroxidase (HRP) (Biogenex, CA, USA) diluted 1:3000 and 3,3',5,5'-tetramethylbenzidine (TMB) substrate (Life Technologies Inc.). The reaction was stopped with $1 \mathrm{M} \mathrm{H}_{2} \mathrm{SO}_{4}$ and the absorbance measured at $450 \mathrm{~nm}$ using a Synergy 2 multi-mode microplate reader and Gen5 software.

\subsubsection{Cathelicidin AMP LPS-binding assay}

The bovine cathelicidin AMPs Bac1, linear Bac1, Bac5, indolicidin, BMAP-28 and BMAP-34 were tested in this assay. The human cathelicidin AMP LL-37 was also tested. A chromogenic Limulus amoebocyte lysate assay kit (Kinetic-QCL assay kit, Lonza, MD, USA) was used to measure cathelicidin AMP-LPS binding. LPS-binding assays were carried out in $5 \mathrm{ml}$ glass tubes, sterilised by heating at $250{ }^{\circ} \mathrm{C}$ for 1 hour. Duplicate titrations of AMP were prepared in endotoxin-free water at a final volume of $54 \mu 1$. LPS (from E. coli strain 055:B5) was reconstituted at $50 \mathrm{EU} / \mathrm{ml}$ in endotoxin-free water and an LPS working dilution of $5 \mathrm{EU} / \mathrm{ml}$ prepared. Each AMP titration was then mixed with $6 \mu$ LPS working dilution, giving a final LPS concentration of $0.5 \mathrm{EU} / \mathrm{ml}$. Standards containing 0.5 EU/ml LPS alone, and negative controls containing endotoxinfree water were also prepared. Tubes were incubated with continuous shaking for 30 minutes at $37{ }^{\circ} \mathrm{C}$. After incubation, $50 \mu \mathrm{l}$ of controls and samples were dispensed into a sterile 96-well tissue culture plate (Nunc) and $50 \mu \mathrm{l}$ of Kinetic-QCL reagent was added. Absorbance was measured at $405 \mathrm{~nm}$ every 90 seconds for 1 hour using a Synergy 2 multi-mode microplate reader and Gen5 software. Free LPS was calculated from absorbance curves for each sample and compared to the $0.5 \mathrm{EU} / \mathrm{ml}$ LPS standards to determine the proportion of LPS bound to AMP. 


\subsection{Microbial assays}

\subsubsection{Microbe preparation}

Preparation of microbe titres was carried out by Megan Callaghan (Food \& Bio-based Products Group, AgResearch, New Zealand). The bacteria Escherichia coli (DNZ-1 isolate, DairyNZ, Newstead, Hamilton), Streptococcus uberis (strain ATCC BAA-854 / 0140J, American Type Culture Collection, VA, USA), Staphylococcus aureus (isolate 2057, isolated in-house, AgResearch, Food \& Bio-based Products Group, New Zealand), Salmonella enterica subspecies enterica (strain ATCC 14028, American Type Culture Collection), were cultured in Mueller-Hinton broth (Fort Richard Laboratories Ltd., Auckland, New Zealand) overnight at $37^{\circ} \mathrm{C}$. The fungus Candida albicans (strain ATCC 10231, American Type Culture Collection) was cultured in either RPMI 1640 medium (Life Technologies Inc.) or Sabouraud B media (Fort Richard Laboratories Ltd.) overnight at $37^{\circ} \mathrm{C}$. A $1 \mathrm{ml}$ aliquot was removed from each strain and centrifuged at 5,000 rpm for 10 minutes. The pellet was washed with $1 \mathrm{ml}$ PBS.

\subsubsection{Microbial agglutination}

Microbes were prepared as described in section 2.5.1 and resuspended in $1 \mathrm{ml}$ PBS containing $0.1 \%$ (w/v) Crystal Violet (BDH Chemicals, Poole, England). Recombinant rbCLD, rbCath1 and native Cath1 protein were titrated 2-fold from $20 \mu \mathrm{g} / \mathrm{ml}$ to $5 \mu \mathrm{g} / \mathrm{ml}$ in $100 \mu \mathrm{l}$ PBS containing $0.1 \%$ Crystal Violet. Control wells contained either no protein or equal amounts of A32 antibody (section 2.2.1) which was used to purify native Cath1. An equal volume of microbe solution was added and agglutination assessed visually after incubating for 1 hour or 3 hours at room temperature using an Olympus CK2 inverted microscope (Olympus America Inc., NY, USA). Agglutination was subjectively judged as absent $(0 \%)$, minimal $(<25 \%)$, moderate $(25-75 \%)$, or severe $(>75 \%)$.

\subsubsection{Minimal inhibitory concentration determination}

The minimal inhibitory concentration (MIC) of a synthetic version of a putative second AMP generated from bovine cathelicidin-1 was investigated in this assay (see section 2.7.1 and 2.7.2). The peptide was tentatively called RITK after the first four amino acids of its sequence (Table 2.1). The MIC assay was based on a standard modified microtitre 
broth dilution method [193]. Microbes were prepared as described in section 2.5.1. S. enterica was omitted from this assay. The microbial cultures were diluted in fresh culture media to give $0.4-1.4 \times 10^{6} \mathrm{cfu} / \mathrm{ml}$. The RITK peptide was diluted in fresh culture media to give two-fold serial dilutions from $200 \mu \mathrm{M}$ to $16 \mu \mathrm{M}$. The microbial dilutions were dispensed into sterile 96-well polypropylene microplates (Nunc) in $50 \mu 1$ duplicates, and $50 \mu \mathrm{l}$ of the peptide serial dilutions were added. Wells containing microbes without peptide served as negative controls and wells containing media alone served as sterility controls. The plates were incubated for 24 hours at $37^{\circ} \mathrm{C}$. After incubation, $1 \mu \mathrm{l}$ from each well was diluted in $1 \mathrm{ml}$ fresh media and plated on MuellerHinton agar plates (Fort Richard Laboratories Ltd.) or Sabouraud dextrose agar plates (Fort Richard Laboratories Ltd.) and incubated for 18 hours at $37{ }^{\circ} \mathrm{C}$. The MIC was determined as the lowest concentration of peptide that prevented any residual colony formation. 


\subsection{Neutrophil function assays}

The method for each neutrophil function assay is provided in this section. Where indicated by italics, each method is subdivided into recombinant protein assays, primary screening assays, or dose-response assays to describe assay modifications. In general, the recombinant protein assays examined rbCLD and rbCath1 activity. As an initial means of identifying bovine cathelicidin AMPs that modify neutrophil function, the primary screening assays examined Bac1, linear Bac1, Bac5, indolicidin, BMAP-28 and BMAP-34 at a fixed concentration of $5 \mu \mathrm{M}$. The peptides which exhibited a significant ability to modify neutrophil function were examined in the dose-response assays. These peptides were linear Bac1, Bac5 and BMAP-34, which were assayed in two-fold titrations from $10 \mu \mathrm{M}$ to $0.16 \mu \mathrm{M}$ unless otherwise indicated.

\subsubsection{Preparation of neutrophils from bovine blood}

Bovine neutrophils were isolated based on a previously described method [194]. Whole bovine blood was obtained from freshly killed cattle at a local abattoir (Ruakura Abattoir, Ruakura Road, Hamilton, New Zealand or Taylor Preston Limited, 131 Centennial Highway, Ngauranga, Wellington, New Zealand) using 10\% (w/v) EDTA as anticoagulant.

Blood was centrifuged at $860 \times \mathrm{g}$ for 30 minutes at $4^{\circ} \mathrm{C}$. Plasma, buffy coat and onethird of the red blood cell (RBC) pellet were removed by aspiration. An equal part icecold sterile MQ. $\mathrm{H}_{2} \mathrm{O}$ was added to the RBC pellet and inverted for 30 seconds to lyse RBCs, followed by an equal part ice-cold 3× RPMI 1640 media to restore tonicity. This procedure was repeated and the cell pellet was washed with ice-cold Hank's Balanced Salt Solution (HBSS) (Life Technologies Inc.) before layering $7 \mathrm{ml}$ of cell suspension over $5 \mathrm{ml}$ Lymphoprep (Medica Limited, Auckland, New Zealand). The density gradient was centrifuged at $800 \times \mathrm{g}$ for 10 minutes at $4{ }^{\circ} \mathrm{C}$. The supernatant containing peripheral blood mononuclear cells was discarded and the granulocyte pellet was washed with ice-cold HBSS and resuspended in appropriate media for experiments.

The concentration of granulocytes was determined by haemocytometer (Neubauer Improved Chamber, Blau Brand, Germany) and the viability was determined by trypan 
blue exclusion ( $0.4 \%$ trypan blue stain, Life Technologies Inc.). To determine purity, cell preparations were stained using a Kwik Diff Stain kit (Thermo Fisher Scientific) and cell types counted using an Olympus BH2 microscope (Olympus America Inc.) and Image-Pro plus v7.0.0.591 software (Media Cybernetics, Inc., USA).

\subsubsection{Detection of apoptotic and necrotic cells}

Neutrophils were plated in duplicate in sterile 2-methacryloxyethyl phosphorylcholine (MCP)-treated 96-well round-bottom plates (Nunc) at $5 \times 10^{5}$ cells/well in RPMI 1640 medium (Appendix 2). The cells were left inactivated (naïve) or were activated with $100 \mathrm{ng} / \mathrm{ml}$ phorbol 12-myristate 13-acetate (PMA) (Sigma-Aldrich) at the same time as AMP treatment.

\section{Primary screening assays}

Neutrophils were treated with $5 \mu \mathrm{M}$ AMP for 1 hour at $37{ }^{\circ} \mathrm{C}$. The cells were then washed with excess RPMI 1640 medium followed by $1 \times$ annexin-V binding buffer (Appendix 2). The cells were stained with FITC-annexin-V (BD Biosciences, CA, USA) diluted 1:100 in $1 \times$ annexin- $V$ binding buffer for 30 minutes on ice. The cells were then washed with PBS and incubated with $1 \mu \mathrm{g} / \mathrm{ml}$ propidium iodide (PI) (SigmaAldrich) for 15 minutes on ice. The cells were washed with ice-cold PBS and kept at $4{ }^{\circ} \mathrm{C}$ until flow cytometry analysis (section 2.6.10).

\section{Dose-response assays}

Neutrophils were treated with titrations of AMP for 1 hour or 6 hours at $37^{\circ} \mathrm{C}$. The cells were then washed with excess RPMI 1640 medium followed by PBS containing $2 \mathrm{mM}$ EDTA to prevent cell clumping. The cells were stained with LIVE/DEAD Fixable Red Dead Cell stain (Life Technologies Inc.) diluted 1:1000 in PBS containing $2 \mathrm{mM}$ EDTA for 30 minutes on ice. The cells were washed with excess $1 \times$ annexin-V binding buffer and incubated with Pacific Blue-annexin-V (BioLegend, CA, USA) diluted 1:200 in annexin-V binding buffer for 30 minutes on ice. The cells were washed with PBS and fixed in PBS containing 4\% (w/v) paraformaldehyde (Fisher BioReagents, NJ, USA) and kept at $4^{\circ} \mathrm{C}$ before preparation for analysis by flow cytometry. 


\subsubsection{Detection of membrane permeabilisation}

Recombinant protein assays

Neutrophils suspended at $1 \times 10^{7}$ cells/ml in PBS were dispensed in duplicate in sterile $5 \mathrm{ml}$ tubes (BD Biosciences) at $1 \times 10^{6}$ cells/tube. For measurement of rbCLD, rbCath1 and native Cath1 membrane permeabilisation, cells were treated with $70 \mathrm{nM}$ or $700 \mathrm{nM}$ rbCLD, rbCath1 or native Cath1 for 30 minutes at room temperature. The cells were stained with $2 \mu \mathrm{g} / \mathrm{ml}$ PI in PBS for 5 minutes at room temperature and placed on ice until analysis by flow cytometry.

\section{Primary screening assays}

Neutrophils were plated in triplicate in sterile 96-well black-wall fluorescence plates (Nunc) at $5 \times 10^{5}$ cells/well in RPMI 1640 medium. The cells were left inactivated (naïve) or were activated with $100 \mathrm{ng} / \mathrm{ml}$ PMA at the same time as AMP treatment. The cells were treated with $5 \mu \mathrm{M}$ AMP and $5 \mu \mathrm{M}$ of the cell impermeant fluorescent dye SYTOX Orange (excitation/emission $547 \mathrm{~nm} / 570 \mathrm{~nm}$ ) (Life Technologies Inc.) for 1 hour at $37^{\circ} \mathrm{C}$. Fluorescence was measured with a Synergy 2 multi-mode microplate reader every 20 seconds for 1 hour with excitation and emission filters set at 528/20 nm and 590/20 nm, respectively. Data were analysed with Gen5 software.

\subsubsection{Migration}

A modified 48-well Boyden chamber kit (Neuro Probe Inc., Gaithersburg, MD, USA) was used for all migration assays. Neutrophils were diluted to $5 \times 10^{6}$ cells $/ \mathrm{ml}$ in Hank's Balanced Salt Solution containing $\mathrm{Ca}^{2+}$ and $\mathrm{Mg}^{2+}\left(\mathrm{HBSS}^{2+}\right)$ (Life Technologies Inc.) with $1 \%(\mathrm{w} / \mathrm{v})$ BSA (referred to as assay media). Recombinant human IL-8 (R\&D Systems, Inc., Minneapolis, MN, USA) diluted to $5 \mathrm{ng} / \mathrm{ml}$ in assay media served as a positive control for migration [40]. Wells containing assay media alone served as negative controls.

\section{Primary screen assays}

Lower wells of the chamber were loaded in duplicate with $5 \mu \mathrm{M}$ AMP in assay media. The chamber was assembled with $3 \mu \mathrm{m}$ pore polycarbonate membrane, gasket and top 
plate as per the instructions provided. The wells of the top plate were loaded with $50 \mu 1$ neutrophil suspension and the chamber was incubated for 45 minutes at $37{ }^{\circ} \mathrm{C}$.

\section{Dose-response assays}

Lower wells of the chamber were loaded in duplicate with titrations of AMP in assay media. The $3 \mu \mathrm{m}$ pore polycarbonate membranes were coated in type I collagen (SigmaAldrich) for 2 hours at $37{ }^{\circ} \mathrm{C}$, rinsed with assay media, and sterilised by UV light exposure for 30 minutes. The chamber was assembled with membrane, gasket and top plate as per the instructions provided. The wells of the top plate were loaded with $50 \mu 1$ neutrophil suspension and the complete chamber was incubated for 1 hour at $37^{\circ} \mathrm{C}$.

After incubation the chamber was disassembled. The top side of the membrane was scraped to remove non-migrated cells and left to air dry. Migrated cells left on the bottom side of the membrane were fixed in $100 \%$ methanol (Fisher Scientific, Loughborough, UK) and stained with Kwik Diff Stain Kit. Stained membranes were mounted under glass coverslips with microscopy immersion oil (Sigma-Aldrich) and the edges were sealed with DPX mountant (BDH Chemicals). Five random microscope fields were counted for each well using a 20× objective on an Olympus BH-2 (Olympus America Inc.) microscope. The mean of duplicates was used to calculate the migration index, defined as the number of migrating cells in response to treatment divided by the number of migrating cells in the absence of treatment.

\subsubsection{Degranulation}

Neutrophils were plated in triplicate in sterile 96-well tissue culture plates at $1 \times 10^{6}$ cells/well in $\mathrm{HBSS}^{2+}$. The cells were left inactivated (naïve) or were activated with $100 \mathrm{ng} / \mathrm{ml}$ PMA at the same time as AMP treatment.

\section{Primary screening assays}

Neutrophils were treated with $5 \mu \mathrm{M}$ AMP for 1 hour at $37{ }^{\circ} \mathrm{C}$. The cells were centrifuged at $1500 \mathrm{rpm}$ for 5 minutes. The supernatant was removed for analysis of primary (section 2.6.5.1) and large (section 2.6.5.3) granule release, and secretory vesicle release (section 2.6.5.4). 
Dose-response assays

Neutrophils were treated with titrations of AMP for 1 hour or 6 hours at $37^{\circ} \mathrm{C}$. The cells were centrifuged at $1500 \mathrm{rpm}$ for 5 minutes. The supernatant was removed for analysis of primary, secondary (section 2.6.5.2) and large granule release.

\subsubsection{Measurement of primary granule release}

Primary granule release was measured by myeloperoxidase (MPO) activity in supernatants [195]. To determine total MPO activity $1 \times 10^{6}$ neutrophils were suspended in an equal volume of $50 \mathrm{mM} \mathrm{KH}_{2} \mathrm{PO}_{4}$ with $0.5 \%(\mathrm{v} / \mathrm{v})$ hexadecyltrimethyl ammonium bromide, sonicated on ice and centrifuged to remove insoluble material. The supernatant was removed and used to form a standard curve of total MPO activity. MPO activity was measured by adding $100 \mu \mathrm{TMB}$ substrate to $50 \mu \mathrm{l}$ supernatant and incubating for 30 minutes at room temperature. The reaction was stopped by adding $50 \mu 11 \mathrm{M} \mathrm{H}_{2} \mathrm{SO}_{4}$. Absorbance was measured at $450 \mathrm{~nm}$ using a Synergy 2 multi-mode microplate reader and Gen5 software.

\subsubsection{Measurement of secondary granule release}

Dose-response assays

Secondary granule release was measured by release of transcobalamin II $[47,196]$ into culture supernatants. Triplicate supernatants were pooled and the protein was concentrated by TCA precipitation (section 2.3.3.1). The precipitated protein was resuspended in $20 \mu \mathrm{l} 3 \times \mathrm{SLB}$ (Appendix 2), boiled for 10 minutes, and analysed by western blot (section 2.3.3.5). Primary antibody incubation was for 3 hours at room temperature using rabbit anti-transcobalamin II antibody (section 2.2.2) diluted 1:10,000 in antibody diluent A (Appendix 2). Secondary antibody incubation was for 1 hour at room temperature using peroxidase-conjugated goat anti-rabbit antibody diluted 1:10,000 in antibody diluent $\mathrm{A}$.

\subsubsection{Measurement of large granule release}

Primary screening assays

Large granule release was measured by release of CLD [45] into culture supernatants. Triplicate supernatants were pooled and $5 \mu$ supernatant was mixed with $10 \mu 13 \times$ SLB, 
boiled for 10 minutes and analysed by western blot (section 2.3.3.5). Primary antibody incubation was for 2 hours at room temperature using rabbit A14 antibody (section 2.2.1) diluted 1:20,000 in antibody diluent A. Secondary antibody incubation was for 1 hour at room temperature using peroxidase-conjugated goat anti-rabbit antibody diluted 1:20,000 in antibody diluent A.

\section{Dose-response assays}

Large granule release was measured by release of CLD [45] into culture supernatants. Triplicate supernatants were pooled and $5 \mu$ supernatant was diluted 20 -fold and analysed by dot blot (section 2.3.3.6). Primary antibody incubation was for 2 hours at room temperature using rabbit A14 antibody diluted 1:20,000 in antibody diluent A. Secondary antibody incubation was for 1 hour at room temperature using peroxidaseconjugated goat anti-rabbit antibody diluted 1:20,000 in antibody diluent A.

\subsubsection{Measurement of secretory vesicle release}

Primary screen assays

Secretory vesicle release was measured by alkaline phosphatase [47] activity in culture supernatants based on a previously described method [197]. Triplicate supernatants were pooled and $33 \mu \mathrm{l}$ supernatant was mixed with $100 \mu \mathrm{l}$-nitrophenyl phosphate liquid (Sigma-Aldrich) in 96-well plates. The plates were incubated for 40 minutes at $37^{\circ} \mathrm{C}$. The reaction was stopped by adding $50 \mu \mathrm{l} 3 \mathrm{M} \mathrm{NaOH}$ and the absorbance was measured at $410 \mathrm{~nm}$ using a Synergy 2 multi-mode microplate reader and Gen5 software.

\subsubsection{Respiratory burst}

\subsubsection{Measurement of extracellular ROS production}

Extracellular superoxide production was measured by reduction of the water-soluble tetrazolium salt, WST-1 (Santa Cruz Biotechnology, Santa Cruz, CA, USA), to a coloured formazan product [198]. Neutrophils were plated in triplicate in 96-well plates at $2 \times 10^{6}$ cells $/ \mathrm{ml}$ in RPMI 1640 medium. The cells were left inactivated (naïve) or were activated with $100 \mathrm{ng} / \mathrm{ml}$ PMA at the same time as AMP treatment. 
Primary screen assays

The cells were treated with $5 \mu \mathrm{M}$ AMP for 1 hour at $37^{\circ} \mathrm{C}$.

\section{Dose-response assays}

The cells were treated with titrations of AMP for 1 hour at $37^{\circ} \mathrm{C}$.

Absorbance was measured at $450 \mathrm{~nm}$ using a Synergy 2 multi-mode microplate reader and Gen5 software.

\subsubsection{Measurement of intracellular ROS production}

The fixable intracellular ROS indicator CellROX Deep Red (excitation/emission $644 \mathrm{~nm} / 665 \mathrm{~nm}$ ) (Life Technologies Inc.) was used to measure intracellular ROS production. Neutrophils were plated in duplicate in MCP-treated 96-well round-bottom plates at $5 \times 10^{5}$ cells/well in RPMI 1640 medium. The cells were left inactivated (naïve) or were activated with $100 \mathrm{ng} / \mathrm{ml}$ PMA at the same time as AMP treatment. The cells were treated with titrations of Bac5-FITC for 5, 15, 30 or 60 minutes in the presence of $1 \mu \mathrm{M}$ CellROX Deep Red. After each time point the cells were washed three times with PBS containing 1\% (w/v) BSA and then fixed in PBS containing $4 \%$ (w/v) paraformaldehyde and kept at $4{ }^{\circ} \mathrm{C}$ before preparation for analysis by flow cytometry (section 2.6.10).

\subsubsection{Phagocytosis}

Phagocytosis was assessed by the uptake of Molecular Probes FluoSpheres (carboxylate-modified microspheres, $0.5 \mu \mathrm{m}$, yellow-green fluorescent (excitation/emission $505 \mathrm{~nm} / 515 \mathrm{~nm}$ ), 2\% solids, Life Technologies Inc.). Fluospheres were opsonised by diluting 1:500 in fetal bovine serum (FBS) (Sigma-Aldrich) and incubating for 1 hour with continuous shaking at $37{ }^{\circ} \mathrm{C}$. Cells treated with $100 \mathrm{ng} / \mathrm{ml}$ PMA served as positive controls for phagocytosis as activated cells have enhanced phagocytic capacity [199]. Cells pre-incubated with $10 \mu \mathrm{M}$ cytochalasin D or B (SigmaAldrich) for 30 minutes at $37^{\circ} \mathrm{C}$ served as negative controls for phagocytosis, as cytochalasin binds to actin and inhibits actin polymerisation [200]. 
Primary screening assays

Neutrophils were plated in duplicate in 24-well tissue culture plates (Nunc) at $5 \times 10^{5}$ cells/well in RPMI 1640 medium. The cells were left to adhere for 30 minutes at $37{ }^{\circ} \mathrm{C}$. The cells were then pre-incubated for 45 minutes with $5 \mu \mathrm{M}$ AMP. FluoSpheres in FBS were added to the cells at a final dilution of 1:50,000 (5.82 spheres/cell) and the cells were incubated for 1 hour at $37{ }^{\circ} \mathrm{C}$. After incubation the cells were fixed in PBS containing $4 \%(\mathrm{w} / \mathrm{v})$ paraformaldehyde and kept at $4^{\circ} \mathrm{C}$ before preparation for analysis by flow cytometry (section 2.6.10).

\section{Dose-response assays}

Neutrophils were plated in duplicate in 96-well MCP-treated round-bottom plates at $5 \times 10^{5}$ cells/well in RPMI 1640 medium. The FluoSpheres were washed in RPMI 1640 medium and added to the cells at a final dilution of 1:50,000 (5.82 spheres/cell). The cells were treated with titrations of AMP for 1 hour at $37^{\circ} \mathrm{C}$. After incubation, the cells were washed with ice-cold PBS and incubated for 2 minutes with ice-cold $0.08 \%(\mathrm{w} / \mathrm{v})$ trypan blue in PBS to quench the fluorescence of surface-bound FluoSpheres. The cells were then washed with PBS to remove excess trypan blue, fixed in PBS containing $4 \%$ (w/v) paraformaldehyde and kept at $4{ }^{\circ} \mathrm{C}$ before preparation for analysis by flow cytometry. Cells were also analysed by confocal microscopy (section 2.9).

\subsubsection{Intracellular calcium mobilisation}

The fluorescent calcium ion indicator Fluo-4/acetoxymethyl ester (Fluo-4 AM, excitation/emission $494 \mathrm{~nm} / 506 \mathrm{~nm}$ ) (Life Technologies Inc.) was used to measure changes in intracellular calcium. Neutrophils were diluted to $2.5 \times 10^{6}$ cells $/ \mathrm{ml}$ in $\mathrm{HBSS}^{2+}$ containing $3 \mu \mathrm{M}$ Fluo-4 AM and mixed for 45 minutes at room temperature. The cells were washed twice in $\mathrm{HBSS}^{2+}$ and incubated for a further 30 minutes at room temperature. The Fluo-4 loaded neutrophils were plated in triplicate in 96-well blackwalled fluorescence plates and pre-warmed to $37^{\circ} \mathrm{C}$. Baseline fluorescence was measured with a Synergy 2 multimode microplate reader with excitation and emission filters set to $485 / 20 \mathrm{~nm}$ and $528 / 20 \mathrm{~nm}$, respectively. The cells were left inactivated (naïve) or were activated with $100 \mathrm{ng} / \mathrm{ml}$ PMA at the same time as AMP treatment. Cells treated with $10 \mu \mathrm{M}$ calcium ionophore A23187 (Sigma-Aldrich) or 10\% zymosanactivated serum (section 2.6.8.1) served as positive controls for calcium mobilisation. 
Primary screen assays

Cells were treated with $5 \mu \mathrm{M}$ AMP and the fluorescence was measured every 20 seconds for 1 hour at $37^{\circ} \mathrm{C}$.

\section{Dose-response assays}

Cells were treated the titrations of AMP and the fluorescence was measured every 20 seconds for 10 minutes at $37{ }^{\circ} \mathrm{C}$ and an endpoint reading was taken after 1 hour at $37{ }^{\circ} \mathrm{C}$.

\subsubsection{Preparation of zymosan-activated serum}

Whole bovine blood was obtained from freshly killed cattle at a local abattoir and collected in $50 \mathrm{ml}$ tubes without anticoagulant. Blood from at least three different cows was collected. The blood was incubated for 1 hour at $37{ }^{\circ} \mathrm{C}$ and then for 1 hour at $20^{\circ} \mathrm{C}$ to allow complete clotting. The tubes were centrifuged at $1500 \times \mathrm{g}$ for 15 minutes at 4 ${ }^{\circ} \mathrm{C}$. The serum supernatant was removed, pooled and centrifuged at $13,000 \times \mathrm{g}$ for 10 minutes at $4{ }^{\circ} \mathrm{C}$. Aliquots of $1 \mathrm{ml}$ were stored at $-80{ }^{\circ} \mathrm{C}$ until use.

Preparation of activated serum was based on the method described by Rainard, et al. [61]. Zymosan A from Saccharomyces cerevisiae (Sigma-Aldrich) was prepared at $2 \mathrm{mg} / \mathrm{ml}$ in HBSS and boiled for 1 hour. The zymosan was washed three times by centrifugation at $1,000 \times \mathrm{g}$ for 2 minutes and resuspended in HBSS. After the third wash the zymosan was resuspended at $2 \mathrm{mg} / \mathrm{ml}$ in pooled serum and incubated with continuous shaking for 2 hours at $37^{\circ} \mathrm{C}$. The zymosan was pelleted by centrifugation at $1,000 \times \mathrm{g}$ for 2 minutes. Activated serum was removed and stored in $1 \mathrm{ml}$ aliquots at $-80{ }^{\circ} \mathrm{C}$ until use.

\subsubsection{Peptide internalisation}

Neutrophils were plated in duplicate in MCP-treated 96-well round-bottom plates at $5 \times 10^{5}$ cells/well in RPMI 1640 medium. The cells were treated with titrations of Bac5FITC for 5, 15, 30 or 60 minutes. After each time point the cells were washed with icecold PBS and incubated for 2 minutes with ice-cold $0.08 \%(\mathrm{w} / \mathrm{v})$ trypan blue in PBS. The cells were then washed with PBS to remove excess trypan blue, fixed in PBS 
containing $4 \%(\mathrm{w} / \mathrm{v})$ paraformaldehyde and kept at $4{ }^{\circ} \mathrm{C}$ before preparation for analysis by flow cytometry (section 2.6.10).

\subsubsection{Flow cytometry analysis}

Neutrophils were gated according to forward scatter and side scatter properties. Where dual staining was applied, positive and negative controls were singly stained and compensation for spectral overlap adjusted accordingly. Generally 10,000 events were collected. However, where a loss of cells occurred due to cytotoxicity, the sample was run until the maximum possible volume was utilised, and the data were analysed according to the number of events collected.

Primary screening assays

Prior to analysis, the cells were resuspended in IsoFlow Sheath Fluid (Beckman Coulter New Zealand Ltd., North Shore City, New Zealand). For detection of apoptotic and necrotic cells, membrane permeabilisation assays and phagocytosis assays, the cells were analysed using a FACS Scan or FACS Calibre flow cytometer with BD CellQuest software v5.2.1 (BD Biosciences Immunocytometry Systems, USA).

Dose-response assays and Bac5 analysis

Prior to analysis, the cells were resuspended in BD FACSFlow Sheath Fluid (BD Biosciences). For detection of apoptotic and necrotic cells, phagocytosis, Bac5-FITC internalisation and intracellular ROS production, the cells were analysed using a FACSVerse flow cytometer and BD FACSuite software v1.0.3 (BD Biosciences Immunocytometry Systems). 


\subsection{Protein interaction assays}

\subsubsection{Protease digestion of rbCath1}

Recombinant cathelicidin-4 (produced by Robert Wieliczko, Food \& Bio-based Products Group, AgResearch, New Zealand) and rbCath1 were concentrated in $100 \mathrm{mM}$ HEPES (pH 7.5) (BDH Chemicals), using Vivaspin 500 centrifugal concentrators with $10 \mathrm{kDa}$ molecular weight cut off. Elastase from human leukocytes (Sigma-Aldrich) was reconstituted in $100 \mathrm{mM}$ HEPES, $\mathrm{pH}$ 7.5. Recombinant protein and elastase were mixed in a ratio of $50 \mu \mathrm{g}$ recombinant protein: $1 \mu \mathrm{g}$ elastase, in a total volume of $50 \mu 1$, and incubated for 30,60 or 90 minutes at $37{ }^{\circ} \mathrm{C}$. A $2 \mu 1$ sample from each digestion was analysed by western blot (section 2.3.3.5). The predicted sequence of the cleavage product contained the epitope for recognition by the cathelicidin-1 CLD-specific A32 antibody (section 2.2.1). Therefore in western blot analysis the primary antibody incubation was for 2 hours at room temperature using rabbit A32 antibody diluted 1:10,000 in antibody diluent A. Secondary antibody incubation was for 1 hour at room temperature using peroxidase-conjugated goat anti-rabbit antibody diluted 1:10,000 in antibody diluent $\mathrm{A}$. The $3 \mathrm{kDa}$ band identified by western blot was also analysed by MS (section 2.8.1) to confirm the peptide sequence.

\subsubsection{Ex vivo production of cathelicidin-1 peptides}

Neutrophils were plated in 12-well tissue culture plates (Nunc) at $5 \times 10^{7}$ cells/well in $\mathrm{HBSS}^{2+}$. The cells were treated with $100 \mathrm{ng} / \mathrm{ml}$ PMA or $10 \mu \mathrm{M}$ calcium ionophore A23187 and incubated with gentle shaking for $1,2,4$ or 8 hours at $37{ }^{\circ} \mathrm{C}$. The culture media was removed and centrifuged at $800 \times \mathrm{g}$ for 5 minutes at $4{ }^{\circ} \mathrm{C}$. The supernatant was removed and frozen at $-80{ }^{\circ} \mathrm{C}$ until liquid chromatography-MS/MS (LC-MS/MS) analysis (section 2.8.2.2).

\subsubsection{Membrane fractionation for $\mathbf{p 4}^{\mathrm{phox}}$ analysis}

Neutrophils were suspended at $5 \times 10^{6}$ cells $/ \mathrm{ml}$ in RPMI 1640 medium and were dispensed in $15 \mathrm{ml}$ tubes (BD Biosciences) at $2 \times 10^{7}$ cells/tube. The cells were left inactivated (naïve) or were activated with $100 \mathrm{ng} / \mathrm{ml} \mathrm{PMA} \mathrm{at} \mathrm{the} \mathrm{same} \mathrm{time} \mathrm{as} \mathrm{AMP}$ treatment. Naïve cells treated with $2.5 \mu \mathrm{M}$ Bac5 showed significant enhancement of respiratory burst, therefore this concentration was used to treat neutrophils for this 
analysis. Conversely, activated cells treated with $10 \mu \mathrm{M}$ Bac5 showed significantly inhibited respiratory burst, therefore this concentration was used to treat activated neutrophils for this analysis. The cells were incubated for 0,30 , or 60 minutes at $37{ }^{\circ} \mathrm{C}$. At each time point the cells were pelleted by centrifugation at $800 \times \mathrm{g}$ for 5 minutes.

The supernatant was discarded and the cells resuspended in $400 \mathrm{ml}$ fractionation buffer (Appendix 2) and lysed by sonication on ice with two 10 second bursts. The lysates were clarified by centrifugation at $13,000 \times \mathrm{g}$ for 10 minutes at $4{ }^{\circ} \mathrm{C}$ and transferred to Nalgene UltraPlus tubes (Nalge Company, NY, USA). Cleared lysates were centrifuged at 35,000 rpm for 60 minutes at $4{ }^{\circ} \mathrm{C}$ using a Type 70.1 Ti rotor (Beckman Coulter) in a Sorvall Discovery 90 SE ultracentrifuge (Hitachi, Japan). The supernatant was removed (cytosolic fraction) and protein concentration was determined by Bio-Rad protein assay (section 2.3.3.2). Cytosolic fractions were stored at $-80{ }^{\circ} \mathrm{C}$ until use.

The membrane pellet was resuspended in $50 \mu 13 \times$ SLB (Appendix 2) and stored at $-80{ }^{\circ} \mathrm{C}$ until use. Membrane protein concentration was determined by SDS-PAGE analysis (section 2.3.3.4). Each lysate was then analysed by western blot (section 2.3.3.5). Primary antibody incubation was for 2 hours at room temperature using rabbit anti-p47 $7^{\text {phox }}$ antibody (sc-7660-R, Santa Cruz Biotechnology) diluted 1:10,000 in antibody diluent A. Secondary antibody incubation was for 1 hour at room temperature using peroxidase-conjugated goat anti-rabbit antibody diluted 1:10,000 in antibody diluent A.

\subsubsection{Preparation of whole neutrophil lysate}

Neutrophil lysates were prepared by reconstituting untreated cells at $2 \times 10^{7}$ cells $/ \mathrm{ml}$ in hypotonic buffer (Appendix 2). The cells were lysed by sonication on ice with three 10 second bursts, followed by two freeze-thaw cycles. The lysates were clarified by centrifugation for 10 minutes at $13,000 \times \mathrm{g}, 4{ }^{\circ} \mathrm{C}$. Protein concentration was determined by Bio-Rad protein assay. The supernatant was stored at $-80{ }^{\circ} \mathrm{C}$ until use. 


\subsubsection{Analysis of Bac5 protein interactions}

Neutrophil lysates prepared in section 2.7.4 were separated by SDS-PAGE in triplicate. Two of the three replicates were analysed in parallel by far-western blot (section 2.3.3.8). For Blot 1, no primary probe was used. Instead, incubation was for 2 hours at room temperature using reagent diluent B (Appendix 2). Secondary probe incubation was for 2 hours at room temperature using Bac5-FITC peptide diluted to $0.5 \mu \mathrm{M}$ in antibody diluent B. For Blot 2, primary probe incubation was for 2 hours at room temperature using unlabelled Bac5 peptide diluted to $2.5 \mu \mathrm{M}$ in antibody diluent $\mathrm{B}$. Secondary probe incubation was for 2 hours at room temperature using Bac5-FITC peptide diluted to $0.5 \mu \mathrm{M}$ in antibody diluent B. The third SDS-PAGE replicate was analysed by LC-MS/MS (section 2.8.2.1) to identify the proteins interacting with Bac5FITC.

\subsubsection{Immunopurification of cellular $\beta$-actin}

The immunopurification method was based on a method developed in-house (AgResearch, Ruakura, New Zealand). A 50\% slurry of Protein A-agarose beads (Sigma-Aldrich) was prepared in hypotonic buffer. The neutrophil lysate was diluted to $0.8 \mathrm{mg} / \mathrm{ml}$ in hypotonic buffer and pre-cleared by adding $50 \%$ slurry at $10 \mu 1 / \mathrm{ml}$ of protein dilution and mixing for 30 minutes at room temperature. The beads were pelleted by centrifugation at 2,000 rpm for 1 minute. The supernatant was transferred to a new tube and $1 \mathrm{ml}$ mixed with $1 \mu \mathrm{g}$ anti-actin antibody (A2066, Sigma-Aldrich) for 16 hours at room temperature. Slurry, freshly washed in hypotonic buffer, was added at $15 \mu \mathrm{l} / \mathrm{ml}$ of protein and mixed for 2 hours. The beads were pelleted as before and the supernatant retained for SDS-PAGE analysis. The beads were washed twice with hypotonic buffer and three times with PBS containing protease inhibitors. The beads were pelleted and resuspended in $20 \mu \mathrm{l} 3 \times \mathrm{SLB}$, boiled for 3 minutes and stored at $-20{ }^{\circ} \mathrm{C}$ until SDS-PAGE and far-western blot analysis (section 2.3.3.8). For far-western blot analysis, primary probe incubation was for 2 hours at room temperature using Bac5-FITC peptide diluted to $0.5 \mu \mathrm{M}$ in antibody diluent $\mathrm{B}$. 


\subsubsection{Co-immunopurification of $\beta$-actin and Bac5-FITC}

The method for co-immunopurification of cellular or purified $\beta$-actin and Bac5-FITC was similar to the method described in section 2.7.6. Exogenous Bac5-FITC was added at a final concentration of $26.1 \mu \mathrm{g} / \mathrm{ml}$ to combinations of neutrophil lysate and antibody (Table 2.3), or purified $\beta$-actin (Huntingtree Bioscience Supplies, Auckland, New Zealand) and antibody (Table 2.4). Combinations were mixed overnight at room temperature and prepared for analysis as in section 2.7.6. Supernatants from the purified $\beta$-actin co-immunoprecipitation were concentrated by TCA precipitation (section 2.3.3.1). Supernatants, TCA precipitates and co-immunopurified protein were separated by SDS-PAGE and analysed as described in section 2.3.3.9.

Table 2.3 Combinations of cellular $\beta$-actin and Bac5-FITC co-immunopurification

\begin{tabular}{|cccc|}
\hline Standard & No lysate & No antibody & No Bac5-FITC \\
\hline 0.8 mg lysate & - & $0.8 \mathrm{mg}$ lysate & $0.8 \mathrm{mg}$ lysate \\
$1 \mu \mathrm{g}$ antibody & $1 \mu \mathrm{g}$ antibody & - & $1 \mu \mathrm{g}$ antibody \\
$26.1 \mu \mathrm{g}$ Bac5-FITC & $26.1 \mu \mathrm{g}$ Bac5-FITC & $26.1 \mu \mathrm{g} \mathrm{Bac5-FITC}$ & - \\
$15 \mu \mathrm{l} 50 \%$ slurry & $15 \mu \mathrm{l} 50 \%$ slurry & $15 \mu \mathrm{l} 50 \%$ slurry & $15 \mu \mathrm{l} 50 \%$ slurry \\
\hline
\end{tabular}

Table 2.4 Combinations of purified $\beta$-actin and Bac5-FITC co-immunopurification

\begin{tabular}{|cccc|}
\hline Standard & No $\beta$-actin & No antibody & No Bac5-FITC \\
\hline $1 \mu \mathrm{g} \beta$-actin & - & $1 \mu \mathrm{g} \beta$-actin & $1 \mu \mathrm{g} \beta$-actin \\
$1 \mu \mathrm{g}$ antibody & $1 \mu \mathrm{g}$ antibody & - & $1 \mu \mathrm{g}$ antibody \\
$26.1 \mu \mathrm{g} \mathrm{Bac5-FITC}$ & $26.1 \mu \mathrm{g}$ Bac5-FITC & $26.1 \mu \mathrm{g} \mathrm{Bac5-FITC}$ & - \\
$15 \mu \mathrm{l} 50 \%$ slurry & $15 \mu \mathrm{l} 50 \%$ slurry & $15 \mu \mathrm{l} 50 \%$ slurry & $15 \mu \mathrm{l} 50 \%$ slurry \\
\hline
\end{tabular}




\subsection{Mass spectrometry}

\subsubsection{MALDI TOF/TOF}

In preparation for MS analysis, polyacrlyamide gels were fixed in MS gel fixative solution (Appendix 2) for 16 hours at room temperature, with two to three changes of fixative solution during this time. Fixed gels were then stained with CBB G-250 stain solution (Appendix 2) for 16 hours at room temperature. The gels were destained with MS gel destain solution (Appendix 2) at room temperature until the background was clear.

After CBB G-250 staining the particular protein band required for analysis was excised. The gel plug was destained overnight at $4{ }^{\circ} \mathrm{C}$ with gel destain solution (Appendix 2), washed for 1 hour with fresh destain wash solution (Appendix 2) and incubated in $25 \mathrm{mM}$ DTT (Roche Diagnostics N.Z., Ltd.) for 30 minutes at $60^{\circ} \mathrm{C}$. After cooling, the gel plug was incubated in $100 \mathrm{mM}$ iodoacetamide (Sigma-Aldrich) in the dark for 1 hour at room temperature. The gel piece was then washed twice for 30 minutes in destain wash solution and dehydrated in pure acetonitrile (Merck, Darmstadt, Germany). The acetonitrile was removed and the gel piece dried by vacuum centrifugation (Savant SpeedVac, Thermo Fisher Scientific New Zealand Ltd.) for 15 minutes.

The dried gel piece was incubated in trypsin digest solution (Appendix 2) for 16 hours at $37{ }^{\circ} \mathrm{C}$ followed by incubation in a sonicating water bath (Fisher Scientific) for 10 minutes. The gel piece was centrifuged and the supernatant removed to a new tube. The pipette tip was retained. The gel plug was resuspended in acetonitrile/TFA solution (Appendix 2) and sonicated for 10 minutes. The supernatant was removed to the new tube using the retained pipette tip. This process was repeated once more, and then repeated again using pure acetonitrile. The pooled supernatant was frozen for 20 minutes at $-80{ }^{\circ} \mathrm{C}$ and lyophilised by vacuum centrifugation. The lyophilised peptides were reconstituted in matrix solution (Appendix 2), spotted onto the MALDI plate and washed with spot wash solution (Appendix 2). Samples from section 2.3.2.4 were analysed by Grant Smolenski (Food \& Bio-based Products Group, AgResearch, New Zealand) using a Bruker Autoflex TOF/TOF mass spectrometer (Bruker Daltonics, 
Bremen, Germany). Samples from section 2.7.1 were analysed by Grant Smolenski using an ABI Q-Star Pulsar i mass spectrometer (Applied Biosystems, MA, USA). Data were analysed using ProteinScape 3.0 (Bruker).

\subsubsection{LC-MS/MS}

\subsubsection{Preparation of Bac5 protein interaction samples}

Samples from the analysis of Bac5 protein interactions (section 2.7.5) were prepared as described in section 2.8.1. The lyophilised peptides were reconstituted in $0.1 \%(\mathrm{v} / \mathrm{v})$ formic acid (Fluka Analytical, Sigma-Aldrich) before analysis. LC-MS/MS analysis was carried out by Irina Boggs (Food \& Bio-based Products Group, AgResearch, New Zealand).

\subsubsection{Preparation of $e x$ vivo produced cathelicidin-1 peptides}

Preparation and analysis by LC-MS/MS was carried out by Ancy Thomas and Stefan Clerens (Food \& Bio-based Products Group, AgResearch, New Zealand). The culture media from stimulated neutrophils (section 2.7.2) was diluted two-fold in $0.1 \%(\mathrm{v} / \mathrm{v})$ formic acid. The solution was transferred to a preconditioned centrifugal filter unit with $10 \mathrm{kDa}$ nominal molecular weight limit (Ultrafree-MC, EMD Millipore). The unit was centrifuged at $14,000 \times \mathrm{g}$ for 30 minutes at $4{ }^{\circ} \mathrm{C}$ and the filtrate retained. A $2 \mathrm{M}$ ammonium formate solution ( $\mathrm{pH}$ 3.0) (Fluka Analytical, Sigma-Aldrich) was added and the unit was centrifuged again. The filtrate was discarded and a solution containing $75 \%(\mathrm{v} / \mathrm{v})$ acetonitrile with $0.1 \%(\mathrm{v} / \mathrm{v})$ formic acid was used to elute bound peptides. The total filtrate was lyophilised in a vacuum centrifuge and reconstituted in $0.1 \%(\mathrm{v} / \mathrm{v})$ formic acid.

\subsubsection{LC-MS/MS analysis}

For each sample, $5 \mu \mathrm{L}$ was loaded at a flow rate of $5000 \mathrm{nl} / \mathrm{min}$ on a Magic C18AQ Nano trap column $(100 \mu \mathrm{m}$ internal diameter $\times 100 \mathrm{~mm}, 5 \mu, 200 \AA)$ (Bruker Daltonics) connected to a Bruker nano-Advance UHPLC equipped with nano-Advance Autosampler. The trap column was then switched in line with a Magic C18AQ analytical column (100 $\mu \mathrm{m}$ internal diameter $\times 150 \mathrm{~mm}, 3 \mu, 200 \AA)$ (Bruker Daltonics) and peptides were eluted with a gradient from $2-45 \%(\mathrm{v} / \mathrm{v})$ acetonitrile in $0.1 \%(\mathrm{v} / \mathrm{v})$ 
formic acid at a flow rate of $800 \mathrm{nl} /$ minute. The analytical column outlet was directly connected to a Bruker Daltonics maXis impact mass spectrometer (Bruker Daltonics) equipped with a CaptiveSpray ion source. Data were analysed using ProteinScape 3.0. 


\subsection{Confocal laser scanning microscopy}

Where indicated, Bac5-FITC was used to visualise internalised Bac5, CellROX Deep Red was used to visualise intracellular ROS production, phalloidin-conjugated tetramethylrhodamine isothiocyanate (TRITC) was used to visualise cellular actin, and 4',6-diamidino-2-phenylindole (DAPI) was used to visualise cell nuclei.

\subsubsection{Slide preparation for phagocytosis analysis}

Aliquots of PMA-treated cells incubated with opsonised FluoSpheres for 1 hour at $37{ }^{\circ} \mathrm{C}$ were dried on microscopy slides. The cells were fixed in PBS containing $4 \%$ (w/v) paraformaldehyde for 30 minutes at room temperature. The cells were washed three times with PBS and mounted using ProLong Gold Antifade Reagent with DAPI (excitation/emission $358 \mathrm{~nm} / 461 \mathrm{~nm}$ ) (Life Technologies Inc.) using \#1.5 coverslips (Warner Instruments, CT, USA). After mounting, the coverslips were sealed with DPX mountant and stored at $-20{ }^{\circ} \mathrm{C}$ until confocal microscopy analysis.

\subsubsection{Slide preparation for colocalisation analysis}

Neutrophils were seeded onto $12 \mathrm{~mm} \# 1.5$ coverslips in 12-well flat-bottom tissue culture plates at $2 \times 10^{5}$ cells/well in RPMI 1640 medium. The cells were allowed to adhere for 15 minutes at $37^{\circ} \mathrm{C}$. For naïve cells, the cells were treated with $2.5 \mu \mathrm{M}$ Bac5-FITC and $5 \mu \mathrm{M}$ CellROX Deep Red for 60 minutes at $37^{\circ} \mathrm{C}$. For activated cells, the cells were treated with $10 \mu \mathrm{M}$ Bac5-FITC, $100 \mathrm{ng} / \mathrm{ml}$ PMA and $5 \mu \mathrm{M}$ CellROX Deep Red for 60 minutes at $37^{\circ} \mathrm{C}$.

After treatment the coverslips were washed four times with excess PBS containing $1 \%(\mathrm{w} / \mathrm{v}) \mathrm{BSA}$ and fixed in PBS containing 4\% (w/v) paraformaldehyde for 30 minutes at room temperature. The coverslips were washed with excess PBS and fixed in acetone for 30 seconds. The cells were then permeabilised for 5 minutes with $0.1 \%$ Triton X-100 (Sigma-Aldrich), washed in excess PBS, and blocked with PBS containing $1 \%(\mathrm{w} / \mathrm{v}) \mathrm{BSA}$ for 1 hour at room temperature. The coverslips were then incubated with $5 \mu \mathrm{g} / \mathrm{ml}$ phalloidin-TRITC (excitation/emission $547 \mathrm{~nm} / 572 \mathrm{~nm}$ ) (Sigma-Aldrich) for 1 hour at room temperature. The coverslips were washed three times with PBS and mounted on slides using ProLong Gold Antifade Reagent with DAPI to identify nuclei. 
After mounting, the coverslips were sealed with DPX mountant and stored at $-20{ }^{\circ} \mathrm{C}$ until confocal microscopy analysis.

\subsubsection{Slide and image analysis}

Slides were analysed using an Olympus Fluoview FV1000 confocal laser scanning biological microscope and Olympus Fluoview v1.7a software (Olympus America Inc.). Spectral based detection was used to image DAPI, FITC/FluoSpheres and TRITC fluorescence and filter based detection was used to image CellROX Deep Red fluorescence (Table 2.5). This approach prevented overlap in the emission spectrums of different fluorophores. Images were analysed using Image-Pro plus v7.0.0.591 and Corel Paint Shop Pro Photo XI v11.20 (Corel Corporation, ON, Canada).

Table 2.5 Confocal microscopy excitation and emission settings

\begin{tabular}{|lccc|}
\hline Fluorophore & Excitation laser & Emission range & Detection type \\
\hline DAPI & $405 \mathrm{~nm}$ & $425 \mathrm{~nm}-475 \mathrm{~nm}$ & Spectral \\
FITC & $488 \mathrm{~nm}$ & $500 \mathrm{~nm}-540 \mathrm{~nm}$ & Spectral \\
TRITC & $561 \mathrm{~nm}$ & $570 \mathrm{~nm}-630 \mathrm{~nm}$ & Spectral \\
CellROX Deep Red & $633 \mathrm{~nm}$ & $>650 \mathrm{~nm}$ & Filter \\
\hline
\end{tabular}




\subsection{Statistical analysis}

All data were analysed using Minitab v15.1.0.0 (Minitab Inc., State College, PA, USA) or Prism v5.0 (GraphPad Software, Inc., CA, USA). One-way analysis of variance (ANOVA) was conducted using Fisher's post hoc test or Tukey's post-test, where appropriate, to compare treatments with controls, or Student's t-test to compare two treatments. 
Chapter 3: Expression and Activity of Recombinant Cathelicidin-1 


\subsection{Introduction}

The Food \& Bio-based Products Group (AgResearch) initially investigated cathelicidins as an early indicator of mastitis in cattle. Data indicated cathelicidins could be detected prior to the onset of clinical symptoms and the concentration demonstrated a high correlation with SCC [108]. Subsequently, native cathelicidin-1 protein (native Cath1) was prepared by immunopurification of the CLD from mastitic milk for further analysis. The purified protein was able to bind LPS, aggregate several mastitis causing species of microorganism and was cytotoxic toward bovine neutrophils (unpublished data, Food \& Bio-based Products Group, AgResearch). These findings were significant as a defined function for this protein has not been clarified. To produce more Cath1 protein for further studies, two expression constructs were created. Firstly, the CLD from bovine cathelicidin-1 was produced (rbCLD) and secondly a full-length cathelicidin-1 was produced with mutation A143 to F143, rendering the C-terminal peptide resistant to neutrophil elastase cleavage (rbCath1). The activity of the purified protein led to the hypothesis that: the rbCLD and rbCath1 proteins will have similar activity to native

\section{Cath1 protein.}

The recombinant proteins would also be useful tools in further functional studies to determine the full role of the CLD during infection. In this chapter, rbCath1 was used to investigate the production of a second peptide from the CLD of cathelicidin-1. The potential secondary cleavage of cathelicidin-1 is of significant interest as this process would generate a novel undescribed bovine cathelicidin AMP. Interestingly, the elastase cleavage site of cathelicidin-1 is 13 amino acids down-stream of the classical aligned cleavage site of the other six bovine cathelicidins (Table 3.1). Therefore it was hypothesised that: further proteolytic processing of cathelicidin-1 occurs, producing a second antimicrobial peptide. 
Table 3.1 Multiple C-terminal sequence alignment of bovine cathelicidins.

\begin{tabular}{|c|c|c|c|}
\hline Protein Name & $\begin{array}{c}\text { UniProt } \\
\text { Entry [91] }\end{array}$ & C-terminal AMP sequence alignment & $\begin{array}{c}\text { CLD } \\
\text { Length }\end{array}$ \\
\hline Cathelicidin-1 & P22226 & ...FDITCNNHQSI ? RITKQPWAPPQAA > RLCRIVVIRVCR & 114 aa \\
\hline Cathelicidin-2 & P19660 & ..FDINCNELQSV > RFRPPIRRPPIRPPFYPPFRPPIRPPIFPPIRP . . & 101 aa \\
\hline Cathelicidin-3 & P19661 & $\ldots$ FDLNCNELQSV > RRIRPRPPRLPRPRPRPLPFPRPGPRPIP . & 101 aa \\
\hline Cathelicidin-4 & P33046 & ...FDLNCNELQSV > ILPWKWPWWPWRRG & 101 aa \\
\hline Cathelicidin-5 & P54229 & $\ldots$ FDITCAVPQSV > GGLRSLGRKILRAWKKYGPIIVPIIRIG & 102 aa \\
\hline Cathelicidin-6 & P54228 & ...INVTCEELQSV > GRFKRFRKKFKKLFKKLSPVIPLLHLG & 102 aа \\
\hline Cathelicidin-7 & P56425 & ..FDITCNNIQSA > GLFRRLRDSIRRGQQKILEKARRIGERIKDIFRG & 102 aa \\
\hline
\end{tabular}

The classical neutrophil elastase cleavage sites are indicated by the '>' symbol. The predicted alternate cleavage site for cathelicidin-1 is indicated with the '?' symbol. aa, amino acids.

\subsection{Aims}

- Transform, express and purify rbCLD and rbCath1 from E. coli.

- Assess the LPS-binding activity, microbial agglutinating activity and cytotoxicity of rbCLD and rbCath1.

- Investigate whether cathelicidin-1 can produce a second AMP. 


\subsection{Results}

\subsubsection{Expression and purification of rbCLD and rbCath1}

\subsubsection{Bacterial expression constructs}

A bacterial expression system was used due to the ease of use, fast culture generation and high yield of protein. The pET-45b(+) vector was used as the expression construct which contains a N-terminal His-Tag coding sequence allowing easy purification, a strong T7lac promoter, an enterokinase cleavage site and a multiple cloning site producing minimal vector-encoded residues at the target sequence fusion site (Figure $3.1 \mathrm{~A}, \mathrm{~B}$ ).

The Cath1_15/13new5 (Figure 3.1 C) and Cath1_NER2 (Figure 3.1 D) expression constructs had been previously prepared by another researcher. The nucleotide sequence for the 114 amino acids of the CLD and 126 amino acids for the full length bovine cathelicidin-1 coding sequence were cloned into the pET-45b(+) vector. The plasmid was sequenced and the insert was confirmed to be correct. In this plasmid vector protein expression is induced by IPTG via the T7lac promoter. Following EK cleavage, the resulting protein will contain an extra Ser-Pro-Asp-Pro sequence at the N-termini of the rbCLD and rbCath1 protein sequences. 
A

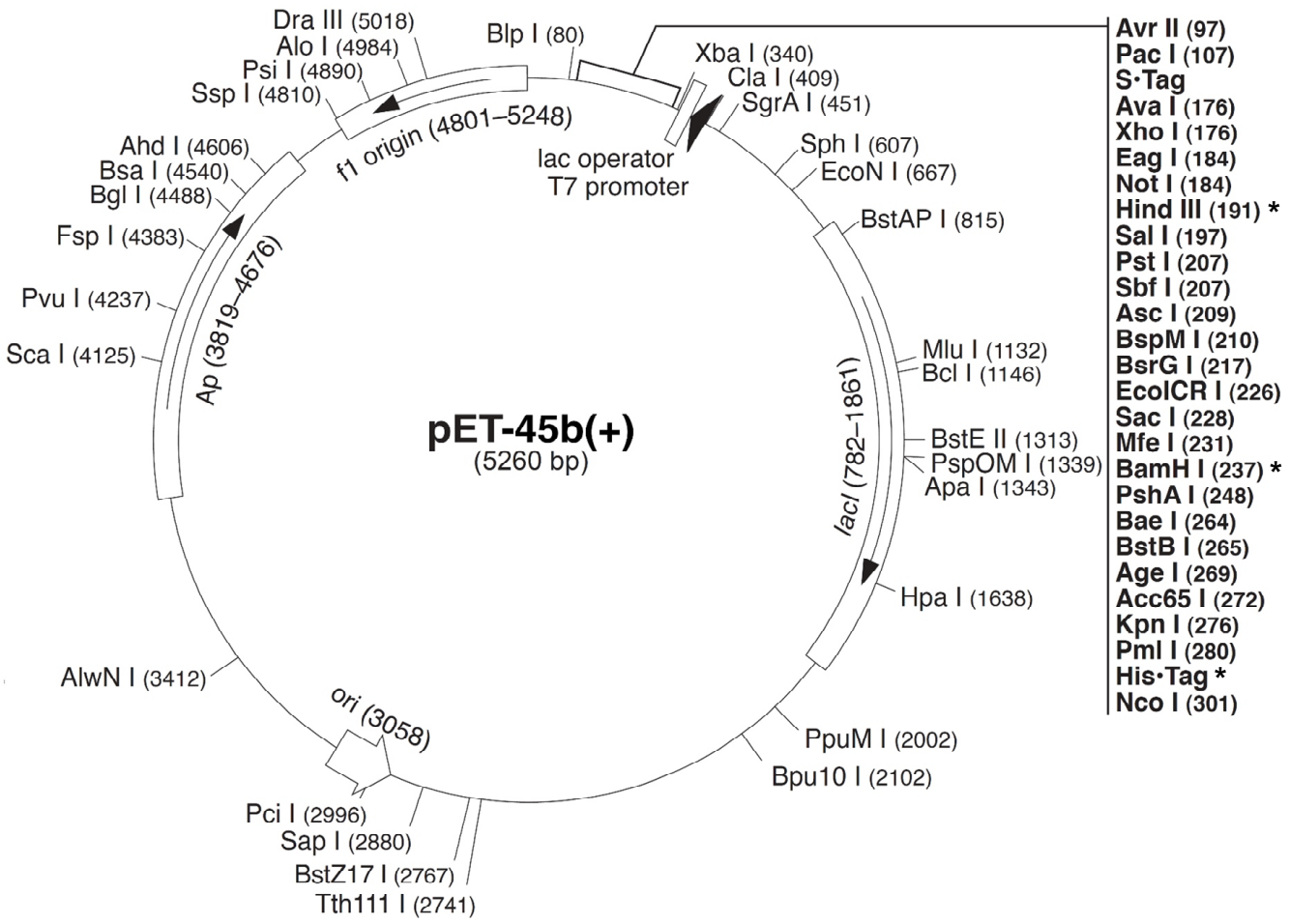

B

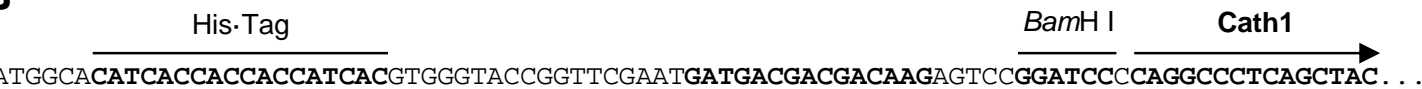
MetAlaHisHisHisHisHisHisValGlyThrGlySerAsnAspAspAspAspLys SerProAspProGlnAlaLeuSerTyr... . enterokinase

C

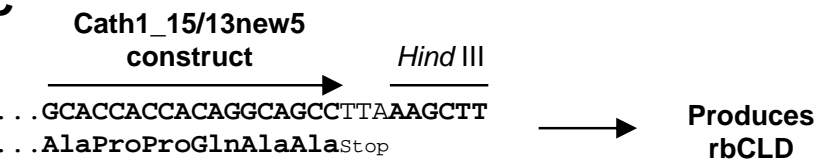

D Cath1_NER2 construct Hind III

. . . GCACCACCACAGGCATTCCGATTATGTCGCATTGTAGTGATAAGGGTTTGCAGATAAAAGCTT

.. AlaP roProGlnAlaPheArgLeuCysArgIleValValI leArgValCysArgstop

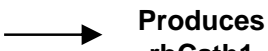
rbCath1 F143 Bac1 AMP

\section{Figure 3.1 Protein coding regions of the constructs expressed in $E$. coli}

(A) Plasmid pET-45b(+) map. (B) Nucleotide and amino acid sequences of the expression region of the pET-45b(+) plasmid. (C) C-terminal of Cath1_15/13new5 construct to express rbCLD. (D) C-terminal of Cath1_NER2 construct to express rbCath1 showing A143 to F143 amino acid substitution to prevent neutrophil elastase cleavage of the AMP domain (Bac1). 


\subsubsection{Small scale expression test for rbCLD and rbCath1}

To ensure the correct protein was translated by each expression construct, a small scale expression test was conducted before scaling up recombinant protein production. Because the CLD contains disulfide bonds, the Origami E. coli strain was used for transformation. This strain contains mutations in the thioredoxin reductase $(\operatorname{tr} x B)$ and glutathione reductase (gor) genes, allowing disulfide bond formation to occur in the bacterial cytoplasm [201]. The $\operatorname{tr} x B$ and gor mutations were selected for by culturing in the presence of kanamycin and tetracycline antibiotics, while the pET-45b(+) plasmid vector was maintained by the presence of ampicillin antibiotic.

The Origami strain was transformed with each plasmid and grew numerous colonies on antibiotic agar plates, whilst control transformation plates showed no growth, indicating a successful transformation. Expression of both plasmids in Origami was induced with IPTG for 90 and 180 minutes (Figure 3.2 A). SDS-PAGE analysis of the expression test indicated induction of significant protein, producing gel bands at approximately $15.5 \mathrm{kDa}$ and $17 \mathrm{kDa}$. This matched the predicted molecular weight of rbCLD and rbCath1 proteins (15.61 $\mathrm{kDa}$ and $17.15 \mathrm{kDa}$, respectively). The bands were confirmed to contain cathelicidin protein by western blot analysis using a cathelicidin-1 specific antibody (Figure 3.2 E, lanes 2 and 3). The rbCLD was mostly soluble after 90 minutes, whereas almost a quarter of total expressed protein was insoluble after 180 minutes of induction. The rbCath1 protein was mostly expressed as insoluble inclusion bodies at both time points. The inclusion bodies were soluble in buffer containing $6 \mathrm{M}$ guanidine hydrochloride.

\subsubsection{Large scale expression and purification of rbCLD}

To yield more recombinant protein, the culture conditions were scaled up to produce two separate batches of $500 \mathrm{ml}$ culture for rbCLD. An expression test using $2 \mathrm{ml}$ of this culture showed minimal 'leaky' expression before induction with IPTG. Nearly all of the induced recombinant protein was soluble (Figure $3.2 \mathrm{~B}$, lane 5) and a negligible amount was insoluble. The recombinant protein was then purified from other bacterial proteins using IMAC. 
The majority of E. coli proteins did not bind to the column during loading and were eluted with the flow through (Figure 3.2 B, lane 9). Minimal recombinant protein was observed in the loading flow through. The first wash removed remaining bacterial proteins with a minor loss of rbCLD. The second wash was virtually clear of bacterial proteins however some high molecular weight protein remained. The yield of rbCLD was high $(4.67 \mathrm{mg} / \mathrm{ml})$ and relatively pure ( 80\% monomeric) but also contained high molecular weight contaminants. The flow through from the first elution was then subjected to a second round of IMAC to determine if the high molecular weight contaminants could be removed (Figure 3.2 C). However, the persistence of these bands in both purifications and their predicted molecular mass indicated these proteins were most likely multimeric forms of the recombinant protein. Western blot analysis confirmed that the bands at approximately $30 \mathrm{kDa}$ were recombinant protein (Figure 3.2 E). The higher molecular weight protein could not be identified.

\subsubsection{Large scale expression and purification of rbCath1}

Culture conditions for rbCath1 were scaled up to $200 \mathrm{ml}$. Due to the formation of inclusion bodies in the small scale test the culture pellet was resuspended in lysis buffer containing $6 \mathrm{M}$ guanidine hydrochloride. Less $E$. coli protein was present in the inclusion bodies and was mostly removed in the flow through of the protein load during IMAC purification (Figure 3.2 D, lane 7). A small amount of recombinant protein was present in the flow through from the protein load and the wash, and no bacterial proteins were visible by CBB R-250 stain after the wash step.

Inclusion bodies are generally considered as aggregated and misfolded protein [202]. On-column refolding was employed to obtain correctly folded protein and remove guanidine hydrochloride from the buffer. This was achieved by immobilising rbCath1 on an IMAC column and slowly replacing the guanidine hydrochloride buffer for buffer without guanidine hydrochloride. No recombinant protein was eluted while the gradient progressed from $0 \%$ to $100 \%$ refold buffer B. The elution step gave a high yield of soluble monomeric rbCath1 $(1.34 \mathrm{mg} / \mathrm{ml})$ with minimal high molecular weight contamination ( $84 \%$ monomeric). Western blot analysis confirmed the presence of a dimer of approximately $34 \mathrm{kDa}$ (Figure $3.2 \mathrm{E}$, lane 3), similar to that observed for the rbCLD purification. 
A

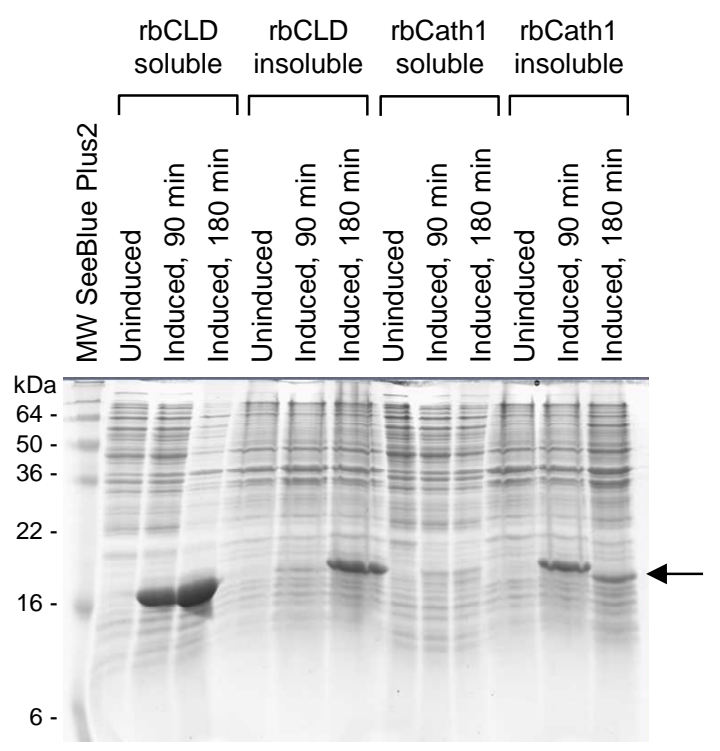

B

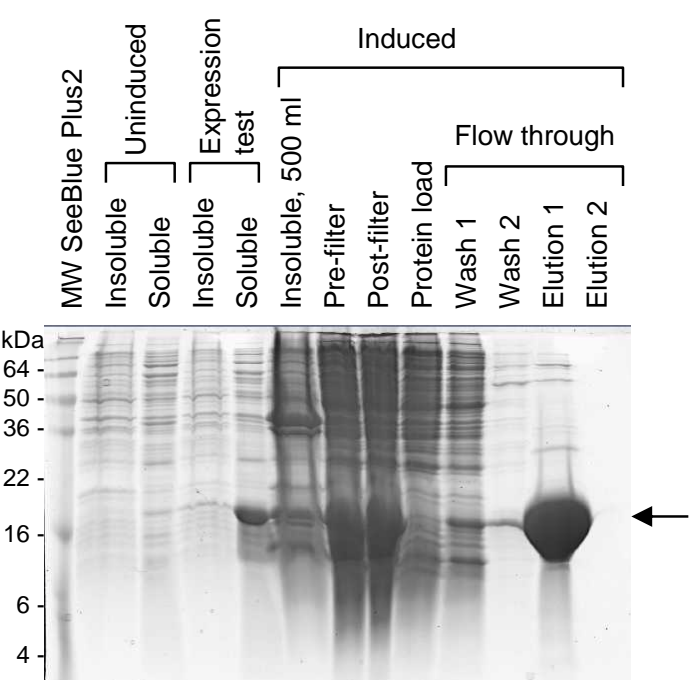

C

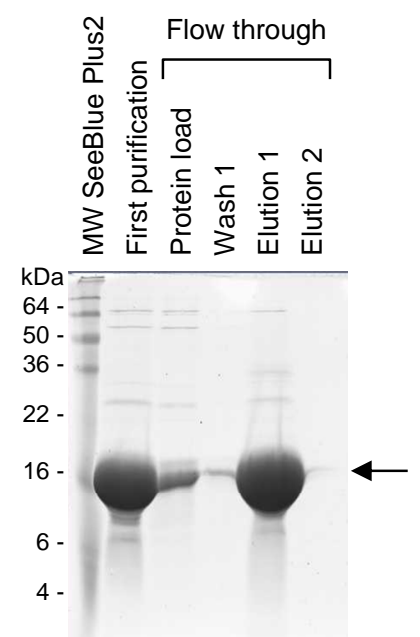

D

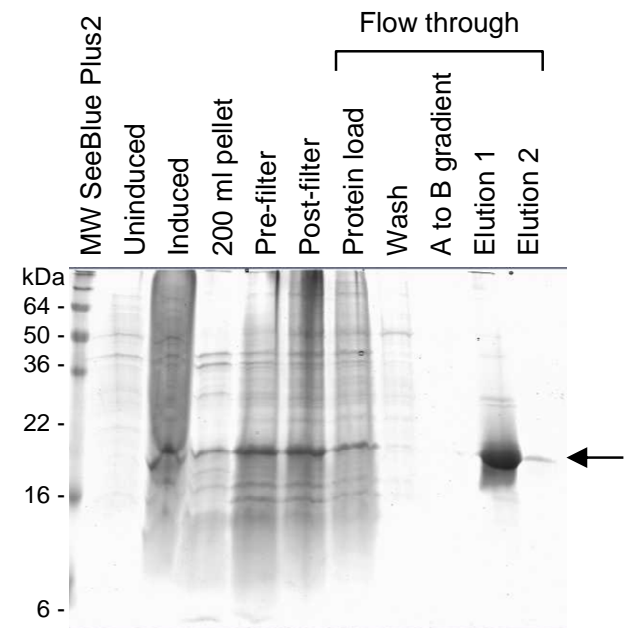

$\mathbf{E}$

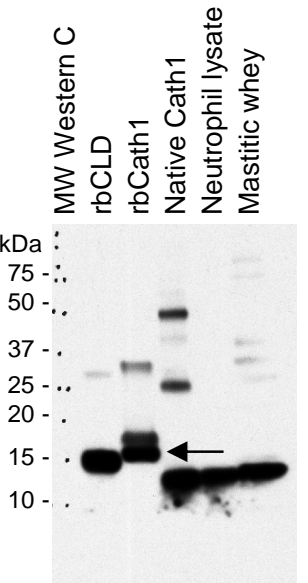

Figure 3.2 SDS-PAGE analysis of the expression and purification of rbCLD and rbCath1

Arrows indicate recombinant protein. (A) Transformed Origami expression test. Cultures were induced with $1 \mathrm{mM}$ IPTG for the indicated time. (B) IMAC purification of rbCLD scaled up to $500 \mathrm{ml}$ culture. Cultures were induced with $1 \mathrm{mM}$ IPTG for 3 hours. (C) Second IMAC purification of rbCLD. (D) IMAC purification of rbCath1 scaled up to $200 \mathrm{ml}$ culture with on-column refolding analysis. Cultures were induced with $1 \mathrm{mM}$ IPTG for 3 hours. (E) Western blot analysis of recombinant and native cathelicidin-1 protein using the cathelicidin-1-specific A32 antibody. 


\subsubsection{Mass spectrometry analysis of rbCLD}

As antibodies only recognise a small antigen sequence, the purified rbCLD was also subjected to MS analysis to ensure the whole protein sequence was translated correctly. An SDS-PAGE of purified rbCLD was stained with colloidal CBB G250. The rbCLD bands were removed and prepared in duplicate for analysis by MALDI LIFT-TOF/TOF [203] (performed by Grant Smolenski, Food \& Bio-based Products Group, AgResearch). Five tryptic peptides were identified for each replicate with statistically significant molecular weight search (MOWSE) probability scores [204] of 67 and 80 respectively. Both samples generated $42 \%$ coverage of the accession sequence, corresponding to 57\% actual coverage (Table 3.2). This confirmed the recombinant protein had the correct sequence.

Table 3.2 Summary of mass spectrometry analysis of rbCLD

\begin{tabular}{|lllcccccc|}
\hline Sample & Identification & Accession & $\begin{array}{c}\text { Weight } \\
\text { (Da) }\end{array}$ & $\begin{array}{c}\text { MOWSE } \\
\text { score }\end{array}$ & Expect & Peptides & Coverage & $\begin{array}{c}\text { Actual } \\
\text { Coverage }\end{array}$ \\
\hline BP-01 & $\begin{array}{c}\text { Cathelicidin-1 } \\
\text { [Bos taurus] }\end{array}$ & gi|27807341 & 17931 & 67 & $\underset{3}{7.2 \times 10}$ & 5 & $42 \%$ & $57 \%$ \\
BP-02 & $\begin{array}{c}\text { Cathelicidin-1 } \\
\text { [Bos taurus] }\end{array}$ & gi|27807341 & 17931 & 80 & $\begin{array}{c}3.4 \times 10 \\
4\end{array}$ & 5 & $42 \%$ & $57 \%$ \\
\hline
\end{tabular}

\subsubsection{Removal of the His· Tag}

The N-terminal His. Tag was removed in order to minimise any differences between the recombinant proteins and native Cath1. This was achieved using an EK cleavagecapture kit. A 48 hour time-course analysis of EK cleavage showed the optimal incubation time was 24 hours at $37^{\circ} \mathrm{C}$ at an enzyme:protein ratio of 1 unit EK:20 $\mu \mathrm{g}$ protein. Although multiple bands of different molecular weight were present, the approximate shift in molecular weight $(\sim 2 \mathrm{kDa})$ corresponded to the expected size of the purification tag (Figure 3.3, lanes 3 and 8). Removal of the cleaved His-Tag by IMAC excessively diluted the target protein, therefore spin-column centrifugation was used to concentrate the flow through containing the target protein. The final purity of cleaved product was approximately $46 \%$ for both recombinant proteins, with yields of $40 \%$ and $9 \%$ of starting protein for rbCLD and rbCath1, respectively. Microdialysis was used to exchange the buffer in preparation for activity assays. 


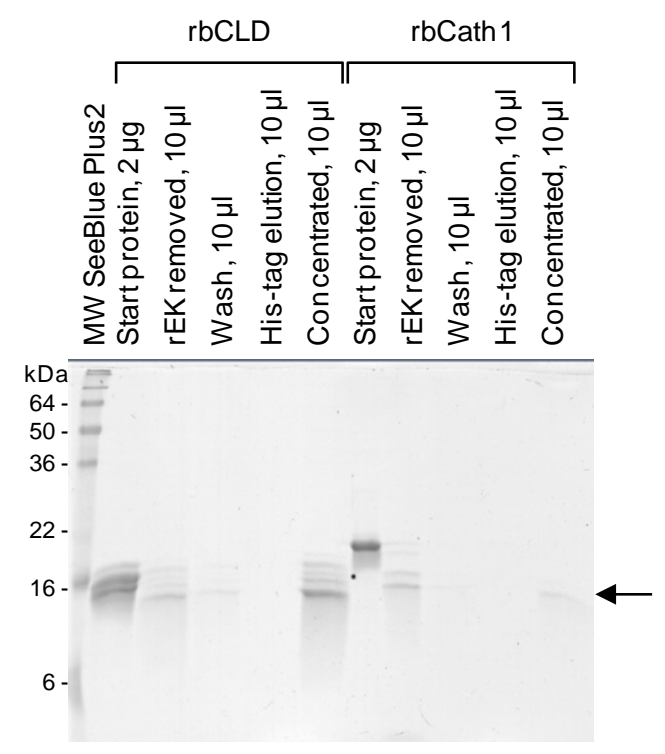

Figure 3.3 Removal of His· Tag from rbCLD and rbCath1

The His·Tag of rbCLD and rbCath1 were removed using a Novagen recombinant enterokinase cleavagecapture kit (EMD Millipore). After removal of the tags the recombinant proteins were concentrated with Vivaspin 6 centrifugal concentrators (Vivaproducts, Inc., USA). 


\subsubsection{Activity and cytotoxicity of rbCLD and rbCath1}

\subsubsection{LPS-binding}

Many immune defence proteins are able to bind LPS, either enhancing or inhibiting its effect on immune cells and tissues [205,206]. An enzyme-linked immunosorbent-based assay was used to test the ability of rbCLD and rbCath1 to bind LPS. Purified bovine LBP served as a positive control, while purified casein served as a negative control. Both recombinant proteins showed comparable LPS-binding capacity at about $20 \%$ of LBP binding (Figure 3.4). This was significantly greater than the casein control. However, the extent to which both recombinants bound LPS was significantly less than that of native Cath1 protein.

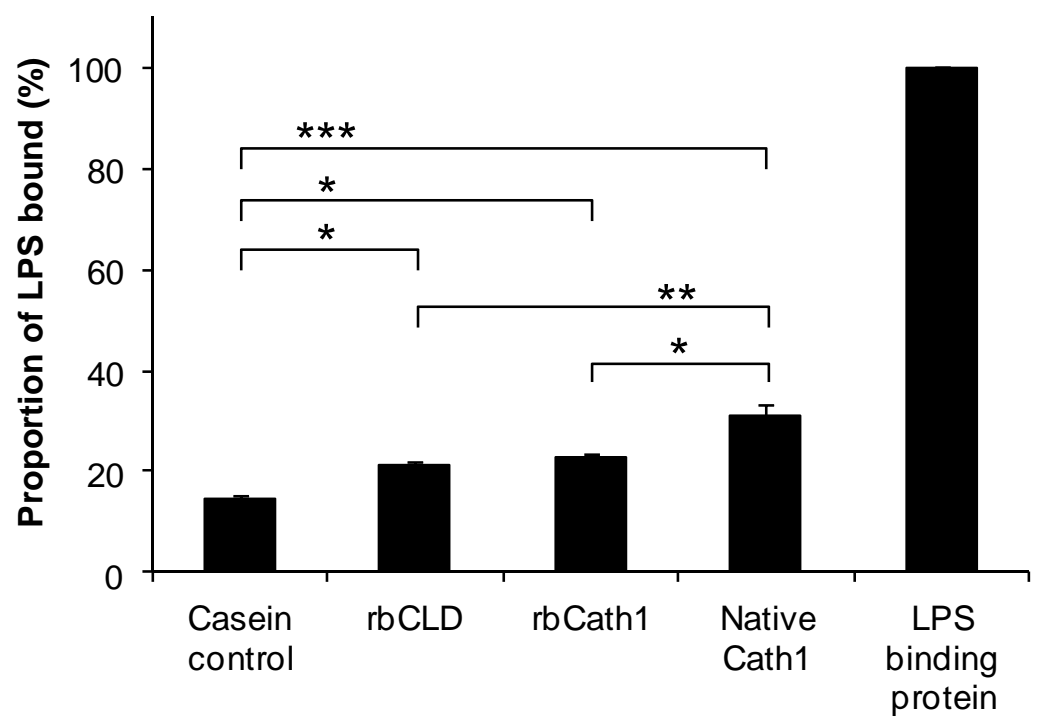

\section{Figure 3.4 LPS-binding activity of rbCLD and rbCath1}

High protein-binding plates (MaxiSorp, Nunc) were coated with $10 \mu \mathrm{g} / \mathrm{ml}$ protein overnight and blocked with $1 \%$ (w/v) casein in PBS. LPS-conjugated biotin was added for 1 hour. Streptavidin-HRP and TMB substrate were used to indicate LPS-binding. Data are expressed as proportion of LPS bound compared to LPS-binding protein $(100 \%)$ and represent the mean \pm SEM for three independent experiments performed in triplicate. Statistical significance was determined by one-way ANOVA using Tukey's post-test. $* P<0.05, * * P<0.01, * * * P<0.001$. 


\subsubsection{Microbial agglutination}

An important feature of innate immune defence is the aggregation and agglutination of microbes. This process prevents the spread of infection and aids clearance by phagocytes. Unpublished data (Food \& Bio-based Products Group, AgResearch) indicates native Cath1 efficiently agglutinates several species of microbe. As far as can be discerned, the agglutinating activity of the bovine CLD has not been reported previously. Several concentrations of rbCLD and rbCath1 were tested against five species of microbe for agglutination activity after 1 hour or 3 hours exposure. In these assays native Cath1 served as a positive control for agglutination. The antibody used to immunopurify native Cath1 (A32 antibody) was also tested as a control for native Cath1 agglutination activity.

Spontaneous agglutination was observed after 1 hour (Table 3.3) and 3 hours (Table 3.4) in all E. coli (Gram-negative) samples. S. typhimurium (Gram-negative) also spontaneously agglutinated after 3 hours. Native Cath1 efficiently agglutinated $S$. uberis (Gram-positive), S. aureus (Gram-positive) and C. albicans (yeast) after 1 hour, which was not attributable to the presence of the A32 purification antibody (control $\mathrm{IgG}$ ). However, neither rbCLD nor rbCath1 was able to agglutinate any of these microbes, even after 3 hours exposure (Table 3.3 and Table 3.4, Figure 3.5 and Figure 3.6). 
Table 3.3 Microbial agglutination scores after 1 hour treatment

\begin{tabular}{|lccccc|}
\hline & E. coli & S. uberis & S. aureus & S. typhimurium & C. albicans \\
\hline Control & ++ & - & - & - & - \\
$10 \mu \mathrm{g} / \mathrm{ml} \mathrm{rbCLD}$ & ++ & - & - & - & - \\
$5 \mu \mathrm{g} / \mathrm{ml} \mathrm{rbCLD}$ & ++ & - & - & - & - \\
$2.5 \mu \mathrm{m} / \mathrm{ml} \mathrm{rbCLD}$ & ++ & - & - & - & - \\
$10 \mu \mathrm{g} / \mathrm{ml}$ rbCath1 & ++ & - & - & - & - \\
$5 \mu \mathrm{g} / \mathrm{ml}$ rbCath1 & ++ & - & - & - & - \\
$2.5 \mu \mathrm{g} / \mathrm{ml}$ rbCath1 & ++ & - & - & - & - \\
$10 \mu \mathrm{g} / \mathrm{ml}$ native Cath1 & ++ & + & +++ & - & +++ \\
$5 \mu \mathrm{g} / \mathrm{ml}$ native Cath1 & ++ & + & +++ & - & +++ \\
$2.5 \mu \mathrm{g} / \mathrm{ml}$ native Cath1 & ++ & + & +++ & - & +++ \\
$10 \mu \mathrm{g} / \mathrm{ml}$ control lgG & ++ & - & - & - & - \\
$5 \mu \mathrm{g} / \mathrm{ml}$ control lgG & ++ & - & - & - & - \\
$2.5 \mu \mathrm{g} / \mathrm{ml}$ control lgG & ++ & - & - & - & - \\
\hline
\end{tabular}

(-) no agglutination; (+) minimal agglutination; (++) moderate agglutination; $(+++)$ severe agglutination

Table 3.4 Microbial agglutination scores after 3 hours treatment

\begin{tabular}{|llcccc|}
\hline & E. coli & S. uberis & S. aureus & S. typhimurium & C. albicans \\
\hline Control & +++ & - & - & + & - \\
$10 \mu \mathrm{g} / \mathrm{ml}$ rbCLD & +++ & - & - & + & + \\
$5 \mu \mathrm{g} / \mathrm{ml} \mathrm{rbCLD}$ & +++ & - & - & + & - \\
$2.5 \mu \mathrm{g} / \mathrm{ml} \mathrm{rbCLD}$ & +++ & - & - & + & - \\
$10 \mu \mathrm{g} / \mathrm{ml}$ rbCath1 & +++ & - & - & + & - \\
$5 \mu \mathrm{g} / \mathrm{ml} \mathrm{rbCath1}$ & +++ & - & - & + & - \\
$2.5 \mu \mathrm{g} / \mathrm{ml}$ rbCath1 & +++ & - & - & + & - \\
$10 \mu \mathrm{g} / \mathrm{ml}$ native Cath1 & +++ & +++ & +++ & + & +++ \\
$5 \mu \mathrm{g} / \mathrm{ml}$ native Cath1 & +++ & +++ & +++ & + & +++ \\
$2.5 \mu \mathrm{g} / \mathrm{ml}$ native Cath1 & +++ & +++ & +++ & + & +++ \\
$10 \mu \mathrm{g} / \mathrm{ml}$ control lgG & +++ & - & - & + & - \\
$5 \mu \mathrm{g} / \mathrm{ml}$ control lgG & +++ & - & - & + & - \\
$2.5 \mu \mathrm{g} / \mathrm{ml}$ control lgG & +++ & - & - & + & + \\
\hline
\end{tabular}

(-) no agglutination; (+) minimal agglutination; (++) moderate agglutination; (+++) severe agglutination 


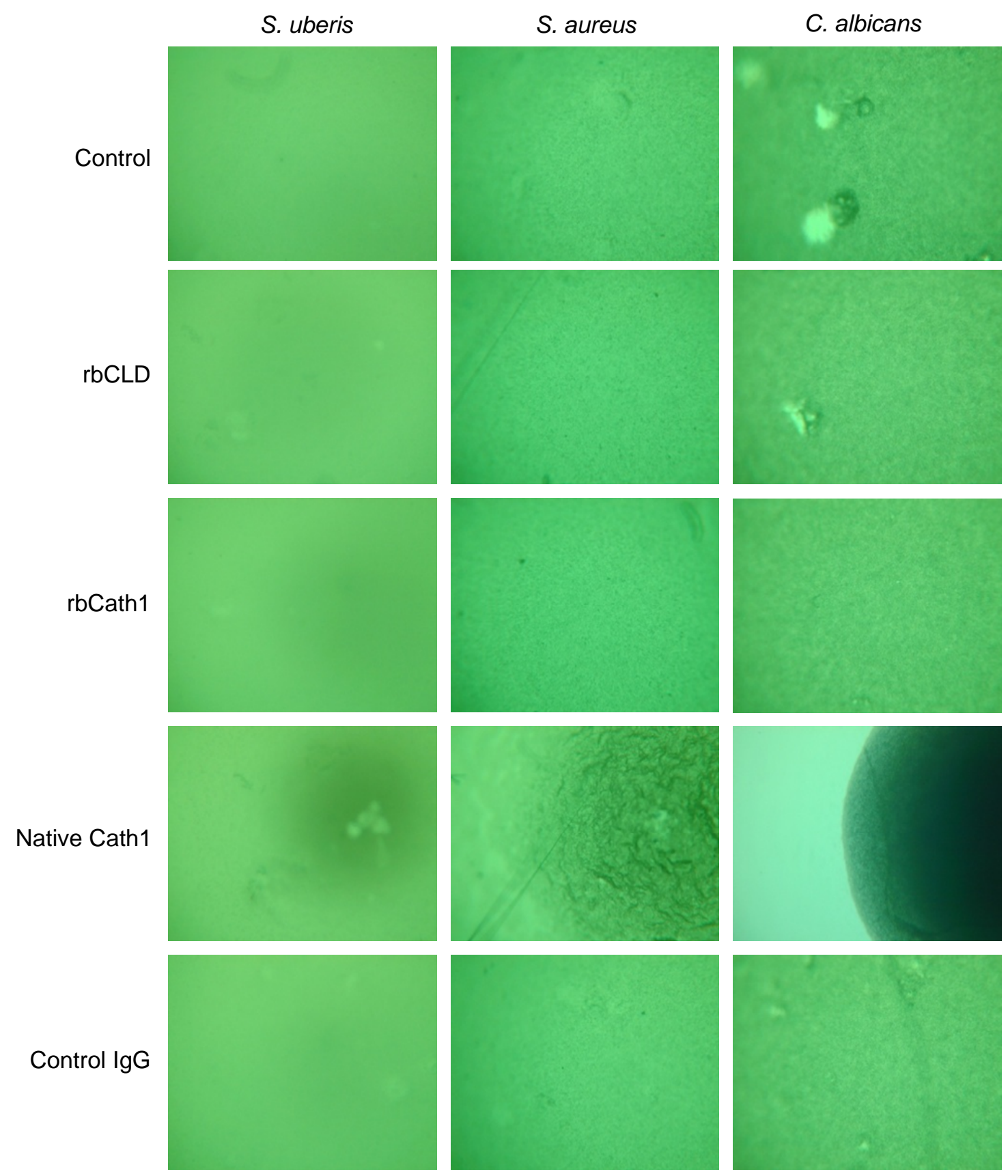

Figure 3.5 Photomicrographs of microbial agglutination after 1 hour treatment

All treatments contain $10 \mu \mathrm{g} / \mathrm{ml}$ protein. Photomicrographs were taken with $40 \times$ objective. 


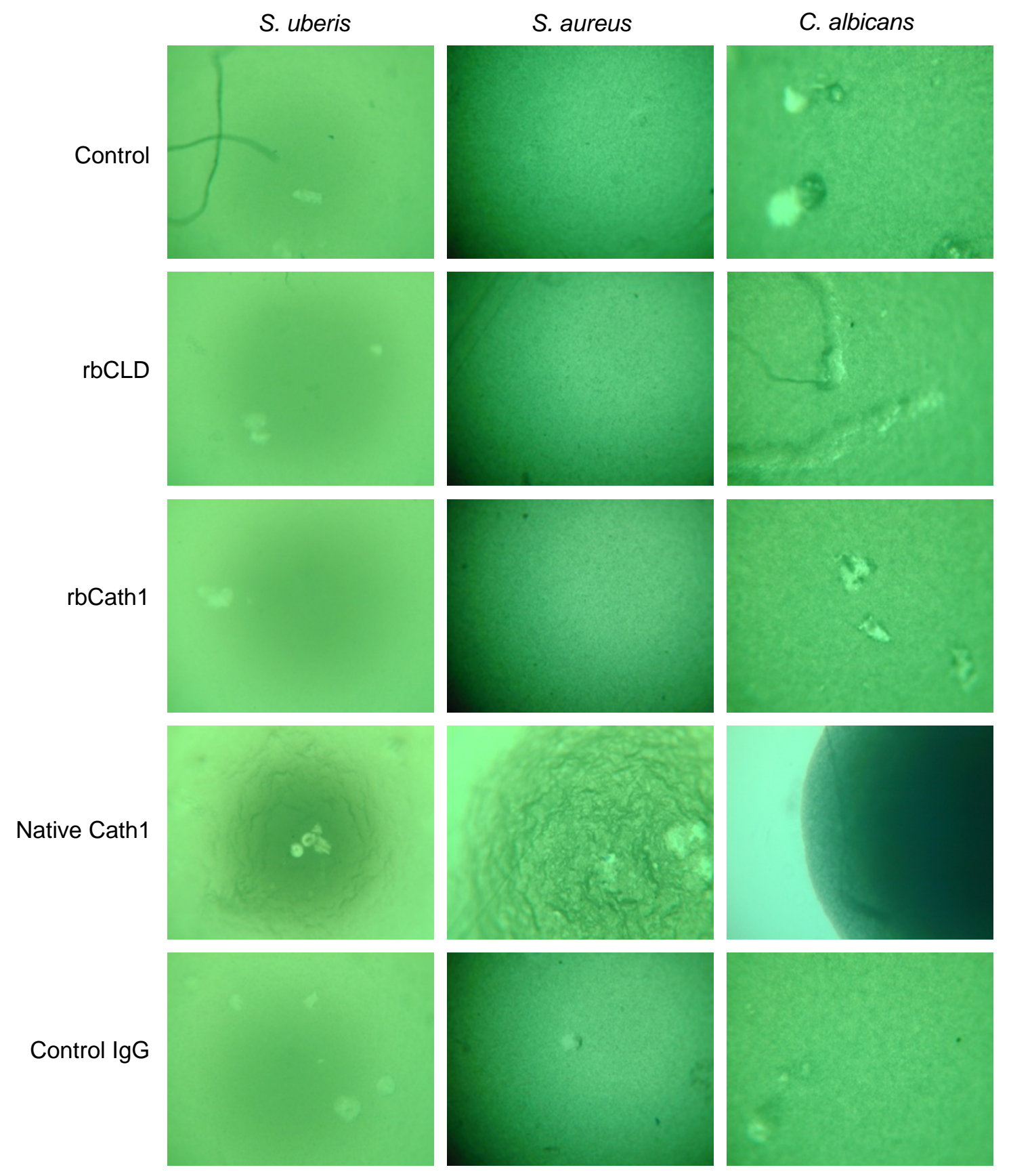

Figure 3.6 Photomicrographs of microbial agglutination after 3 hours treatment

All treatments contain $10 \mu \mathrm{g} / \mathrm{ml}$ protein. Photomicrographs were taken with $40 \times$ objective. 


\subsubsection{Neutrophil cytotoxicity}

Because cathelicidins are constituents of neutrophil granules and released by neutrophils during infection, rbCLD and rbCath1 were tested for cytotoxicity in a neutrophil membrane permeabilisation assay. Unpublished data (Food \& Bio-based Products Group, AgResearch) indicates native Cath1 is cytotoxic to bovine neutrophils at greater than $100 \mathrm{nM}$. Therefore naïve neutrophils were exposed to $70 \mathrm{nM}(\sim 0.91 \mu \mathrm{g} / \mathrm{ml})$ and $700 \mathrm{nM}(\sim 9.1 \mu \mathrm{g} / \mathrm{ml})$ concentrations of rbCLD, rbCath1 and native Cath1 for 30 minutes and stained with PI prior to flow cytometry analysis. The data showed neutrophils were not permeabilised by either rbCLD or rbCath1 at either of the concentrations tested (Figure 3.7). In contrast, native Cath1 was able to permeabilise neutrophil membranes at $700 \mathrm{nM}$ inducing death in nearly all of the cells analysed.

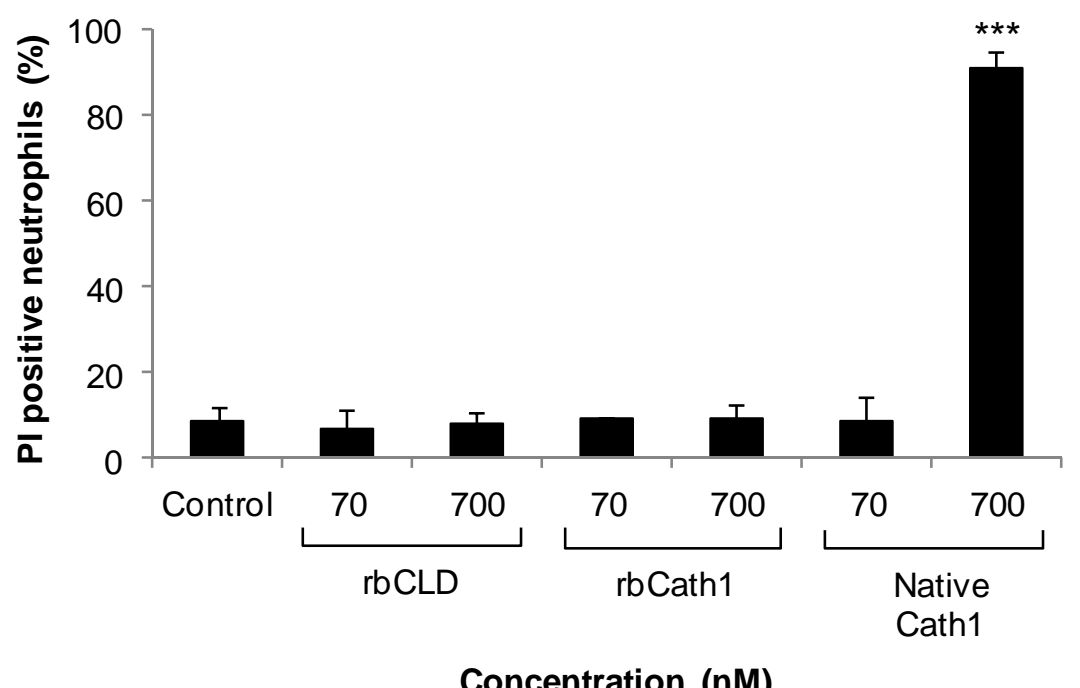

\section{Figure 3.7 Neutrophil membrane permeabilisation}

Bovine neutrophils were exposed to rbCLD, rbCath1 and native Cath1 at the indicated concentrations for 30 minutes at room temperature, stained with PI, and analysed by flow cytometry. Data represent the mean \pm SEM for at least two independent experiments performed in duplicate. Statistical significance was determined by one-way ANOVA using Tukey's post-test comparing treatments with control. $* * * P<0.001$. 


\subsubsection{Investigation of a second AMP produced by cathelicidin-1}

\subsubsection{Digestion of rbCath1 with neutrophil elastase}

The bovine cathelicidins are proteolytically cleaved by neutrophil elastase (NE) to release the AMP domain. The cleavage site is conserved for all expressed bovine cathelicidins except cathelicidin-1. Here the cleavage site is distal to the conserved cleavage site, leaving 13 amino acids at the C-terminus of the CLD (see Table 3.1). This suggested that an alternate cleavage site might exist which could generate an additional novel bovine AMP. Using the MEROPS database [207], this second potential cleavage site was assessed for compatibility with known NE cleavage sites. The most preferential substrates for NE in the $P 1$ position of the scissile bond were valine, isoleucine and alanine (in order). Therefore NE was used to determine whether rbCath1 would be cleaved between $1130(P 1)$ and R131 (P1'), in alignment with the site at which the other bovine cathelicidins are processed. Due to the NE resistant mutation (A143 to F143) the predicted cleavage product would also contain the Bac1 peptide and have an approximate molecular weight of $3 \mathrm{kDa}$. The CLD of bovine cathelicidin-4 (rbCLD4) was produced as a recombinant protein in E. coli (by Robert Wieliczko, Food \& Biobased Products Group, AgResearch) and was used as a negative control for non-specific CLD cleavage.

NE cleavage of rbCath1 revealed a time dependent increase in a low molecular weight product over 90 minutes (Figure $3.8 \mathrm{~A}$ ). No cleavage products of rbCLD4 were visible after Ponceau S stain, indicating cleavage was specific for rbCath1. Western blot analysis using the epitope specific A32 antibody revealed robust production of a $3 \mathrm{kDa}$ peptide from rbCath1 (Figure 3.8 B). CBB G-250 stain was able to resolve the $3 \mathrm{kDa}$ band after SDS-PAGE analysis (Figure 3.8 C), but also revealed several other major products of neutrophil elastase cleavage. The $3 \mathrm{kDa}$ band was removed and subjected to MS analysis. 
A

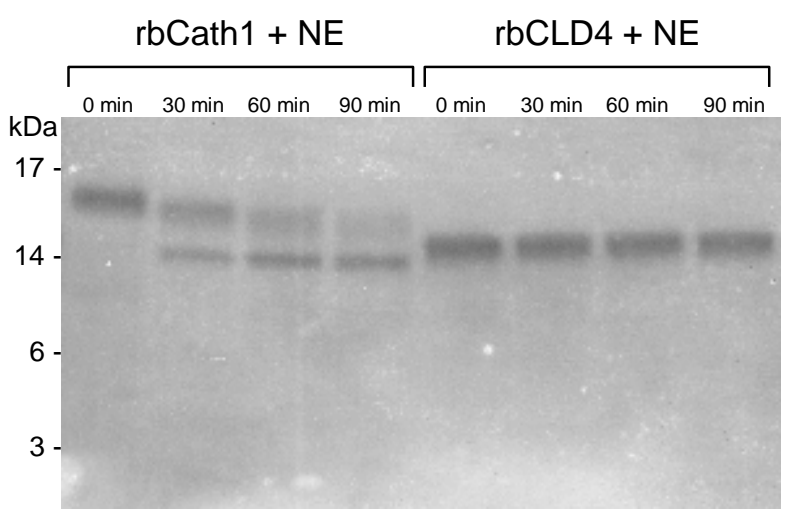

B

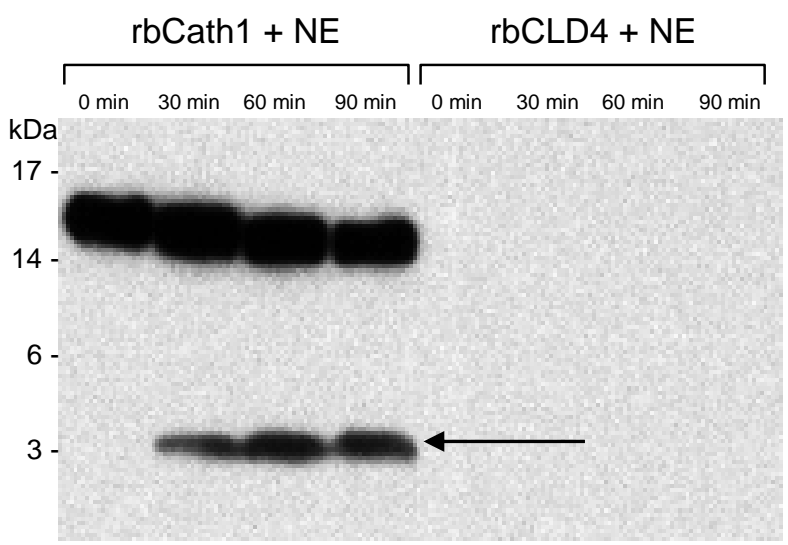

C

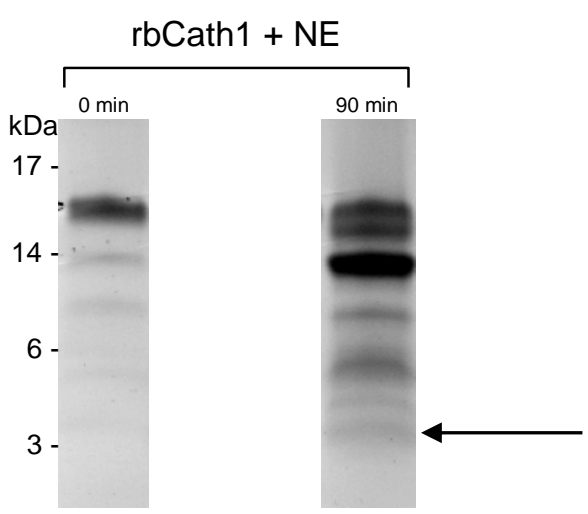

\section{Figure 3.8 Cleavage of rbCath1 by neutrophil elastase}

Recombinant rbCath1 was incubated with NE at $37{ }^{\circ} \mathrm{C}$ for the indicated times and cleavage products were analysed by western blot. Recombinant rbCLD4 served as a negative control for non-specific CLD cleavage. Arrows indicate the predicted $3 \mathrm{kDa}$ product. (A) PVDF transfer membrane stained with Ponceau S. (B) PVDF transfer membrane probed with cathelicidin-1-specific A32 antibody. (C) CBB G-250 stain of SDS-PAGE. 


\subsubsection{MS analysis of the $3 \mathrm{kDa}$ band}

The trypsin-derived peptide fragments of the $3 \mathrm{kDa}$ cleavage product of rbCath1 were used to generate a peptide mass fingerprint mass spectrum using MALDI LIFTTOF/TOF MS analysis [203] (performed by Grant Smolenski, Food \& Bio-based Products Group, AgResearch). Two peptides were identified in the spectrum with MOWSE probability scores of 43.8 and 52.4. Further fragmentation of the peptides using post-source decay produced spectra with good sequence coverage of the mutation site of rbCath1 (Figure $3.9 \mathrm{~A}, \mathrm{~B}$ ). This confirmed the $3 \mathrm{kDa}$ cleavage product had the predicted sequence of R131 to R155. The putative second cathelicidin-1 peptide (with sequence RITKQPWAPPQAA) was hereafter referred to as the RITK peptide.

\subsubsection{MS analysis of stimulated neutrophil culture media}

Having confirmed that the RITK peptide could be produced in vitro, it was then necessary to establish whether the peptide could be produced by bovine neutrophils $e x$ vivo. Isolated neutrophils were stimulated in culture with PMA or calcium ionophore for up to eight hours to promote release of granule contents and maturation of AMPs. Supernatants were removed for analysis by LC-MS/MS (performed by Stefan Clarens and Ancy Thomas, Food \& Bio-based Products Group, AgResearch) to identify the RITK peptide (Figure 3.10 A, B). Synthetic versions of the RITK peptide and Bac1 were used to calibrate the instrument. Bac1 was detected in greater concentrations as treatment time progressed, indicating that endogenous neutrophil elastase processing does occur ex vivo. However, the RITK peptide could not be detected after either treatment at any of the time points measured. 

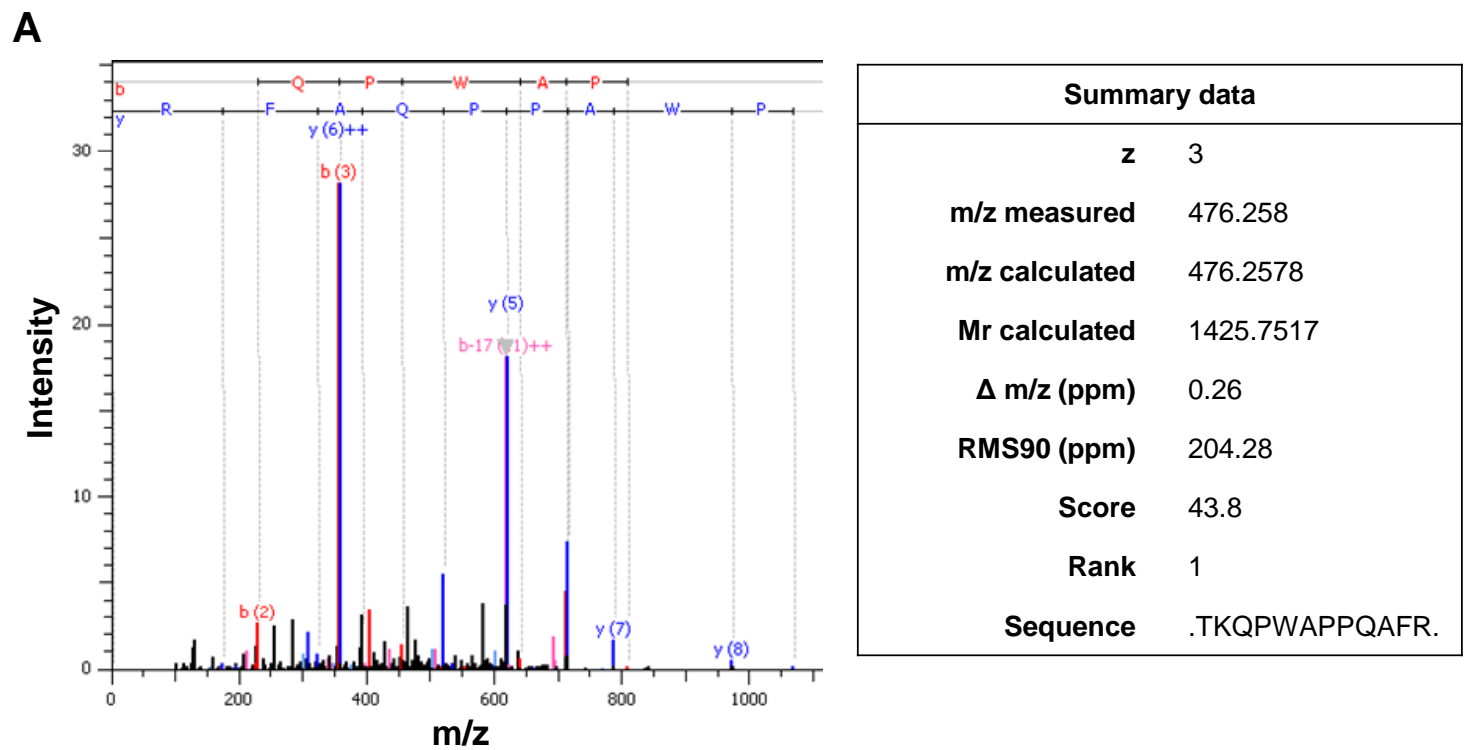

B

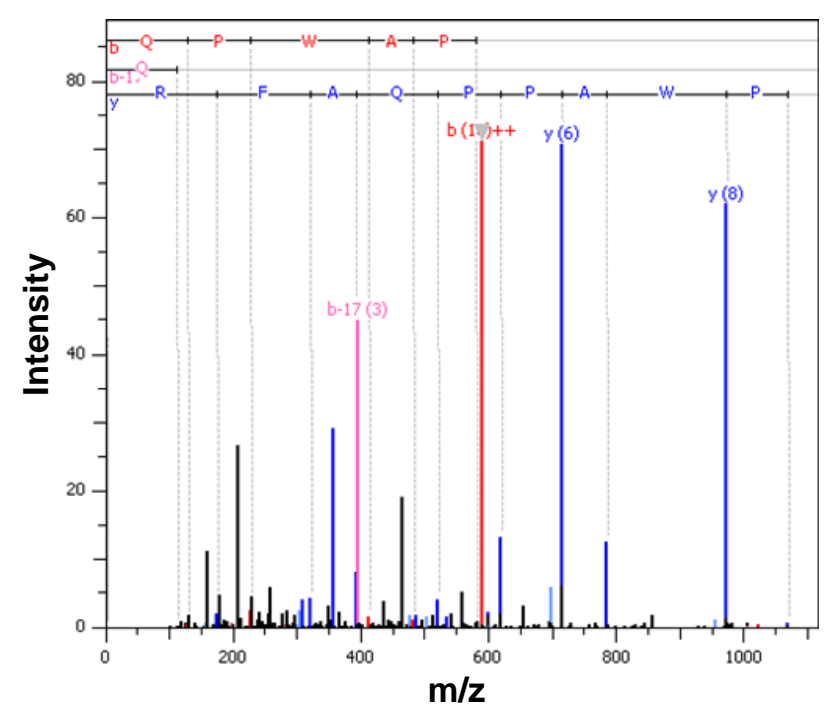

\begin{tabular}{|rl|}
\hline \multicolumn{2}{|c|}{ Summary data } \\
$\mathbf{z}$ & 2 \\
$\mathbf{m} / \mathbf{z}$ measured & 599.3102 \\
$\mathbf{m} / \mathbf{z}$ calculated & 599.3118 \\
$\mathbf{M r}$ calculated & 1196.6091 \\
$\mathbf{\Delta} \mathbf{~} / \mathbf{z}(\mathbf{p p m})$ & -2.66 \\
$\mathbf{R M S} \mathbf{0}(\mathbf{p p m})$ & 44.02 \\
Score & 52.4 \\
Rank & 1 \\
Sequence & K.QPWAPPQAFR.L \\
\hline
\end{tabular}

Figure 3.9 Peptide mass fingerprint data after post-source decay for the $3 \mathrm{kDa}$ cleavage product

Fragmentation mass spectra generated from MALDI LIFT-TOF/TOF analysis. (A) and (B) represent two peptides identified after post-source decay which cover the A to F mutation in the expected $3 \mathrm{kDa}$ cleavage product. 
A
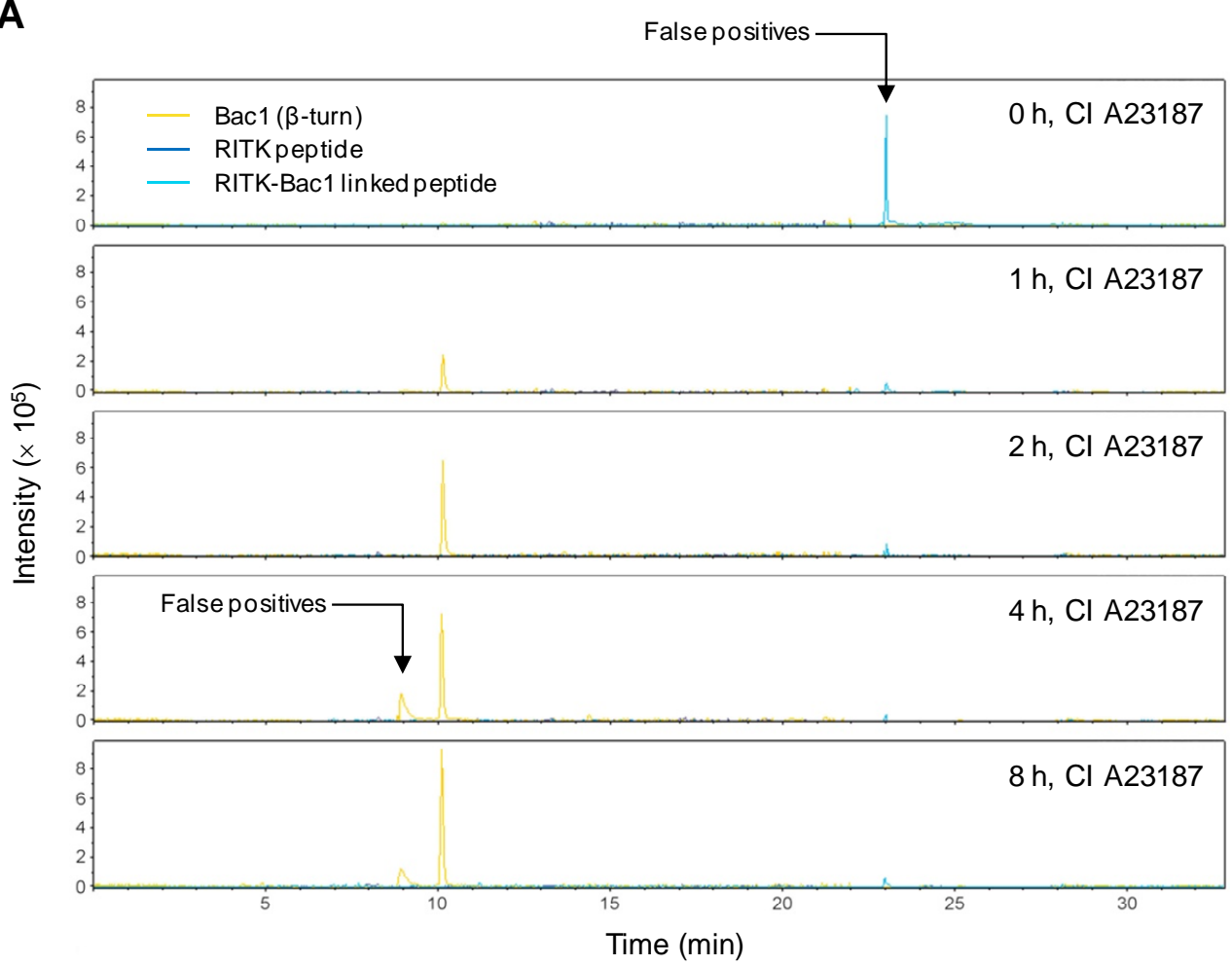

B
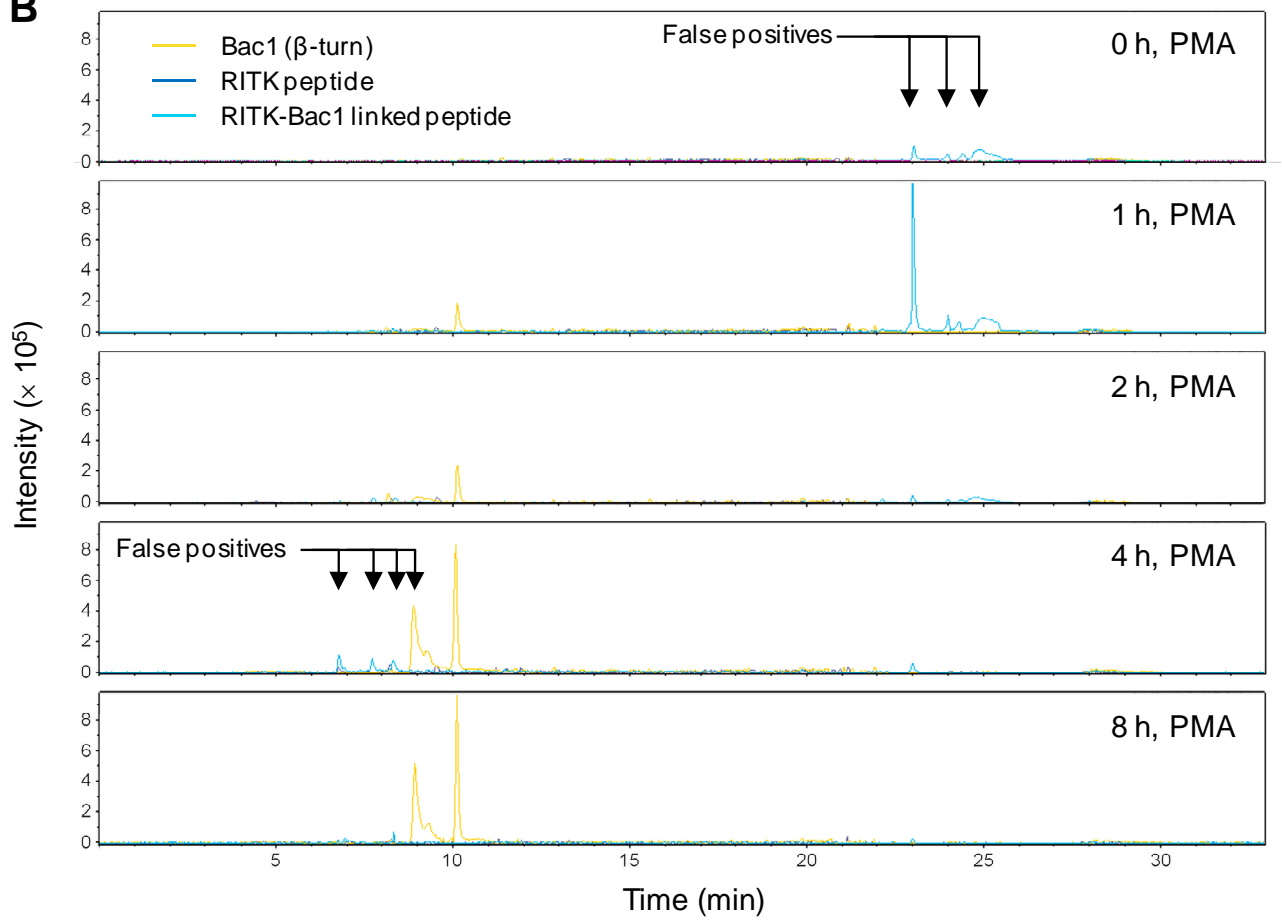

Figure 3.10 LC chromatograms of stimulated neutrophil culture media

Neutrophils were stimulated with (A) $10 \mu \mathrm{M}$ calcium ionophore (CI A23187) or (B) $100 \mathrm{ng} / \mathrm{ml}$ PMA for the indicated times. Culture plates were centrifuged and the supernatants were removed and analysed by LC-MS/MS. No signal for the RITK peptide (dark blue) was observed after either type of stimulation. False positives have either the wrong charge state or different actual mass and charge. 


\subsubsection{Investigation of RITK peptide activity}

\subsubsection{MIC determination}

As AMPs generally have strong antimicrobial activity, a synthetic version of the potentially novel RITK peptide was tested in a standard MIC assay. Several species of microbe were exposed to titrations of the peptide for 24 hours to determine the MIC of the RITK peptide for each microbe.

The RITK peptide was tested at two-fold titrations from $100 \mu \mathrm{M}$ but was unable to inhibit the growth of either E. coli or $S$. aureus after 24 hours at any concentration (data not shown). The growth of $C$. albicans was also uninhibited in Sabouraud B medium and RPMI 1640 medium.

\subsubsection{Neutrophil respiratory burst}

Some AMPs have no intrinsic antimicrobial activity but may have the ability to bind specific receptors and illicit immune responses in certain cell types. For example fMLP [32] can activate human neutrophils via the fPRL-1 receptor, inducing ROS production and chemotaxis $[150,152]$. Therefore the RITK peptide was tested for modulation of respiratory burst in naïve or activated bovine neutrophils but was unable to modify the respiratory burst of cells in either state (Figure $3.11 \mathrm{~A}, \mathrm{~B}$ ). 
A

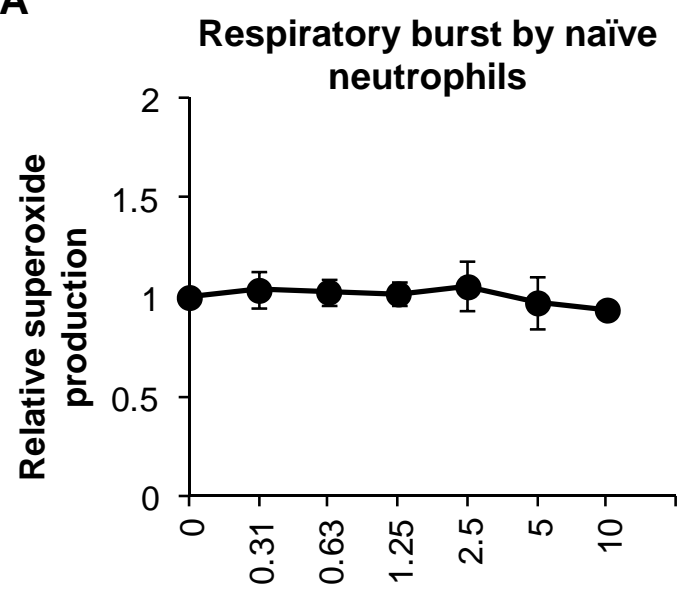

Concentration $(\mu \mathrm{M})$
B
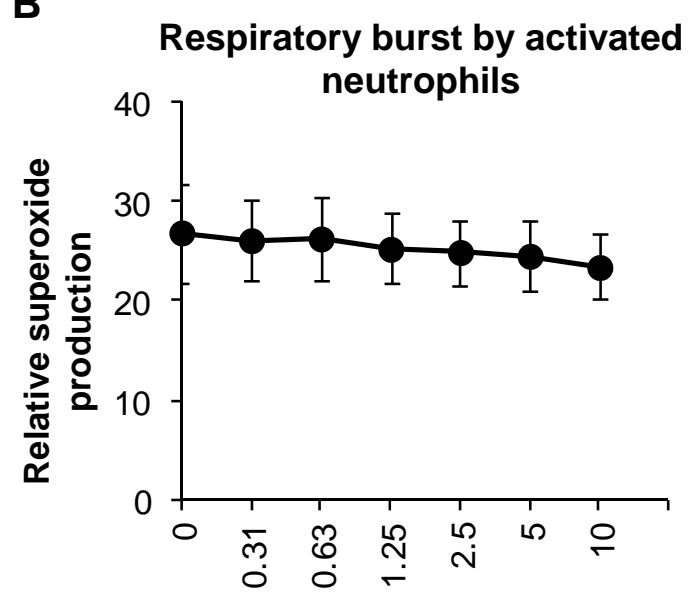

Concentration $(\mu \mathrm{M})$

Figure 3.11 The effect of the RITK peptide on bovine neutrophil respiratory burst

Extracellular superoxide production was tested using the colourimetric dye WST-1 and the absorbance was measured after 1 hour. (A) Naïve cells and (B) cells activated with $100 \mathrm{ng} / \mathrm{ml}$ PMA. Data represent the mean WST-1 reduction relative to naive controls \pm SEM for two independent experiments performed in triplicate. No statistical significance was found using a one-way ANOVA with Tukey's post-test comparing treatments with untreated controls $(0 \mu \mathrm{M}$ AMP). 


\subsection{Discussion}

The aims of the experiments in this chapter were to produce rbCLD and rbCath1 and compare the humoral activity and cytotoxicity to a native purification of Cath1. Additionally, rbCath1 was used to investigate whether cathelicidin-1 could produce a second AMP through cleavage by neutrophil elastase.

\subsubsection{Expression and activity of rbCLD and rbCath1}

A high yield of predominantly soluble monomeric rbCLD was produced in E. coli. However, rbCath1 was expressed as insoluble inclusion bodies which required solubilisation with a strong chaotropic agent and subsequent refolding. The CLD contains two conserved disulfide bonds (C56-C67 and C78-C95). These require the correct pairing and orientation when overexpressed in E. coli in order for the protein to fold correctly. As rbCLD was produced as soluble protein when overexpressed, the presence of a third disulfide bond (C117-C125) in the AMP domain of rbCath1 is likely to have caused the inclusion bodies to form. Several methods are available to attempt to overcome inclusion body formation [208], however in this case adequate soluble protein was produced by refolding rbCath1.

Native Cath1, rbCLD and rbCath1 were able to bind LPS with significantly greater affinity than the casein control, but with only 20-30\% of the capacity of LBP. Despite this comparatively low binding capacity, cathelicidin is relatively abundant during infection [108] which could translate into a large overall capacity to sequester LPS during infection. Binding of LPS and other pathogen associated molecular patterns (PAMPs) can also aid agglutination of microbes. However, rbCLD and rbCath1 were unable to aggregate any microbe tested, whereas native Cath1 was able to efficiently aggregate $C$. albicans and the Gram-positive bacteria $S$. uberis and $S$. aureus. This suggests that native Cath1, at least, may have affinity for peptidoglycans such as lipotechoic acid, a major constituent of Gram-positive bacterial cell walls. Gramnegative bacteria appeared to be unaffected by any treatment, consistent with the LPSbinding data, although $E$. coli could not be adequately tested due to spontaneous agglutination. Further binding assays with alternate types of PAMPs would clarify these observations. 
As agglutination of microbes can aid clearance by phagocytes it is interesting to observe the cytotoxic nature of native Cath1 toward bovine neutrophils. This may indicate a non-specific mode of action such as aggregation at the membrane [209] leading to membrane permeabilisation. As CLD concentration can reach $100 \mu \mathrm{g} / \mathrm{ml}(\sim 10 \mu \mathrm{M})$ [108] in an inflammatory environment this may serve to regulate excessive neutrophil activity and prevent the spread of pathogens.

A question remains as to why rbCLD and rbCath1 were not able to exhibit the same activity as the native Cath1 preparation. Western blot analysis revealed a high molecular weight A32-positive band in the native Cath1 preparation at approximately $50 \mathrm{kDa}$ (see Figure 3.2 E). Preparation of proteins for SDS-PAGE involves boiling protein solutions in denaturing buffer. The presence of this high molecular weight CLD indicates that tetrameric forms or aggregates of the protein may be produced in vivo, which have strong intermolecular bonds inaccessible to the denaturants in sample loading buffer. Only dimeric structures appeared to be formed during recombinant overexpression, and none of these higher molecular weight structures were present in neutrophil lysates. This indicates a structural rearrangement may occur in vivo, after cathelicidin is released during infection. Structural analysis of the porcine protegrin-3 CLD indicates domainswapped dimers can be formed [97] and high molecular weight forms, either multimers or aggregates of cathelicidins, have been observed previously [58,110]. No posttranslational modifications have been reported for the CLD therefore it is possible that the activity observed with the native form is due to the formation of multimeric complexes of the CLD that for some unforeseen reason do not occur with the recombinant proteins. Further analysis of the different molecular weights of native Cath1 protein would clarify if this activity is specific to certain multimers.

\subsubsection{The RITK peptide}

The use of rbCath1 in the investigation of a second peptide of cathelicidin-1 generated a $3 \mathrm{kDa}$ fragment which was resolved by SDS-PAGE and identified by Western blot. MS analysis confirmed the peptide had the predicted sequence indicating the RITK peptide might be produced in vivo. Neutrophils were stimulated to release cathelicidins and the culture media was analysed by LC-MS/MS. However, the RITK peptide could not be identified in this ex vivo experiment. 
There are many factors that may have contributed to this outcome. In particular, the in vitro activity of the bovine neutrophil elastase is reported to have 50 times less activity compared to the human protease when cleaving the synthetic substrate peptidyl-7amino-4-methylcoumarin [210]. A BLAST sequence alignment shows 67\% homology between bovine and human neutrophil elastase with identical catalytic residues but altered active site residues [211]. This might explain why the bovine protease has less activity on synthetic substrates compared to the human protease, and why production of the RITK peptide was not observed ex vivo. However, Bac1 was able to be detected in culture media, demonstrating neutrophil elastase activity was present. If the RITK peptide is actually produced at all, it may be possible that it is only generated within the phagosome and is not produced extracellularly. Further methods toward clarifying the existence of the RITK peptide are discussed in Chapter 6, section 6.2.2.

While the existence of a secondary peptide from cathelicidin-1 was not confirmed, the inability of the synthetic peptide to inhibit microbial growth was not unexpected. Multiple peptide fractions of similar molecular weight were separated when Bac1 was first identified [86] which may have included the RITK peptide. The fraction later found to contain Bac1 was of particular interest because of the strong antimicrobial activity it exhibited. Other peptide fractions were overlooked due to their inability to inhibit microbial growth. Some AMPs have also been identified which lack intrinsic antimicrobial activity but are able to modify innate immunity [212,213]. Despite this, the RITK peptide was unable to modify neutrophil respiratory burst, however this was only one assay of one cell type, therefore possible functions for this peptide remain open. Clarification of the existence of the peptide in vivo will validate whether further functional research is practical. 


\subsection{Conclusions}

Two recombinant versions of the cathelicidin- 1 host defence protein were produced by over expression in E. coli. The recombinants bound less LPS, were unable to agglutinate microbes, and were unable to permeabilise neutrophil membranes when compared to native Cath1. A possible explanation for this is the existence of a high molecular weight multimer of cathelicidin in the native Cath1 preparation which was not observed in the recombinant protein preparations.

A second peptide was generated by in vitro proteolytic cleavage of the C-terminal of rbCath1. Stimulation of neutrophils ex vivo produced detectable Bac1 peptide but did not produce detectable RITK peptide. Different approaches to this experiment, such as isolating and recombining the protease- and cathelicidin-containing granule subsets may yield evidence to the contrary. A synthetic version of the putative 13 amino acid RITK peptide was not able to inhibit microbial growth, nor was it able to modulate neutrophil respiratory burst. However, this does not rule out other immunomodulatory functions for the peptide. The established bovine cathelicidin AMPs do have antimicrobial activity and cathelicidin AMPs from other species are able to modulate immune cell function. The possibility that the bovine cathelicidin AMPs modulate key bovine neutrophil functions was investigated in Chapter 4. 
Chapter 4: Modulation of Bovine Neutrophil Function by Cathelicidin AMPs 


\subsection{Introduction}

\subsubsection{Neutrophil functions}

Neutrophil recruitment and activation is a vital part of the first line of defence against invading pathogens. Upon activation, neutrophils engage a number of methods for effective host defence, including migration, respiratory burst, phagocytosis, and the release of granules containing cathelicidin AMPs and other humoral components [214]. Cathelicidin peptides are considered to act primarily as antimicrobial components. However, there is evidence that AMPs can also regulate inflammation [215], immune cell function [102,154,155], and elicit different effects on naïve and activated cells $[160,216]$. These peptides are from a range of species, each having different structures and unique sequences. Bovine express seven cathelicidin AMPs and each have significantly different sequences and structures. The ability of AMPs from other species to regulate immune cell function and the existence of seven different bovine AMPs led to the hypothesis that: bovine cathelicidin AMPs differentially modulate naïve and activated bovine neutrophil functions.

\subsubsection{LPS-binding activity}

Principal investigations of cathelicidin AMPs have focused on their antimicrobial activity, a component of which involves binding PAMPs. The most common PAMP associated with Gram-negative bacteria is LPS. The LPS-binding capacity of host defence peptides and proteins can vary significantly within and between species due to hydrophobicity, charge and structure [217-220]. LPS sequestration can translate into immune modulation through prevention of TLR activation [178] and inhibition of downstream intracellular signalling. Results from Chapter 3 indicated the CLD was able to weakly bind LPS. However, due to the variation in structure, sequence and net charge of the different bovine AMPs, the ability to bind LPS might be different for each peptide, leading to the hypothesis that: bovine cathelicidin AMPs vary in their ability to bind LPS. 


\subsection{Aims}

- Investigate the modulatory effects of bovine AMPs on neutrophil innate immune function.

- Measure LPS-binding capacity of a representative selection of bovine cathelicidin AMPs. 


\subsection{Results}

\subsubsection{Neutrophil isolation}

Neutrophils were isolated by hypotonic lysis of RBCs and density gradient centrifugation to separate granular cells from peripheral blood mononuclear cells. This technique reliably isolated neutrophils with $>95 \%$ viability as determined by trypan blue exclusion. On average, isolated cells were $96 \%$ neutrophils and $4 \%$ eosinophils.

\subsubsection{Primary screen of neutrophil function}

\subsubsection{Cathelicidin AMP selection}

Five representative bovine cathelicidins, as well as a linear variant of the Bacl $\beta$-turn peptide, were selected for initial testing based on their difference in structure and composition (Table 4.1). Bac1 is the smallest AMP identified to date and may exist in a linear conformation in vivo [86,221,222], therefore both Bac1 variations were selected to identify effects attributable to either structure. Bac5 contains a repeating proline-rich motif (PPXX, where X indicates any amino acid) found in other cathelicidins [223] and has a relatively high net positive charge. Conversely, the linear peptide indolicidin is unique across species, containing an unusually high content of tryptophan [69]. BMAP-28 has an $\alpha$-helical structure with an unstructured hydrophobic C-terminus, while BMAP-34 is larger, contains negatively charged amino acids and forms an amphipathic $\alpha$-helix.

Table 4.1 Properties of the selected bovine cathelicidin AMPs

\begin{tabular}{|c|c|c|c|c|c|c|}
\hline Protein & AMP & Structure & Sequence & $\begin{array}{c}\text { Weight } \\
\text { (kDa) }\end{array}$ & $\begin{array}{c}\text { Net } \\
\text { charge }\end{array}$ & Feature \\
\hline Cathelicidin-1 & Bac1 & $\beta$-turn & RLCRIVVIRVCR & 1.5 & +4 & $\begin{array}{l}\text { Disulfide } \\
\text { bond }\end{array}$ \\
\hline Cathelicidin-1 & Bac1 & Linear & RLCRIVVIRVCR & 1.5 & +4 & $\begin{array}{c}\text { Free } \\
\text { cysteines }\end{array}$ \\
\hline Cathelicidin-2 & Bac5 & $\begin{array}{l}\text { Linear/poly- } \\
\text { proline helix }\end{array}$ & $\begin{array}{l}\text { RFRPPIRRPPIRPPFYPPFR } \\
\text { PPIRPPIFPPIRPPFRPPLG } \\
\text { PFP-NH }\end{array}$ & 5.1 & +9 & $\begin{array}{l}\text { Proline and } \\
\text { arginine rich }\end{array}$ \\
\hline Cathelicidin-4 & Indolicidin & Linear & ILPWKWPWWPWRR-NH ${ }_{2}$ & 1.9 & +3 & $\begin{array}{l}\text { Tryptophan } \\
\text { rich }\end{array}$ \\
\hline Cathelicidin-5 & BMAP-28 & a-helical & $\begin{array}{l}\text { GGLRSLGRKILRAWKKYG } \\
\text { PIIVPIIRI-NH }{ }_{2}\end{array}$ & 3.1 & +7 & $\begin{array}{l}\text { Hydrophobic } \\
\text { C-terminus }\end{array}$ \\
\hline Cathelicidin-7 & BMAP-34 & a-helical & $\begin{array}{l}\text { GLFRRLRDSIRRGQQKILE } \\
\text { KARRIGERIKDIFR-NH }{ }_{2}\end{array}$ & 4.2 & +8 & Amphipathic \\
\hline
\end{tabular}


The physiological concentration of bovine AMPs during infection has not been reported. Therefore the bovine cathelicidin peptides were first tested at a concentration of $5 \mu \mathrm{M}$, consistent with the concentrations at which human LL-37 [150] and porcine PR-39 [154] are reported to exhibit immune activity. Neutrophils recruited to sites of inflammation are readily activated therefore the response of both naïve and PMAactivated neutrophils in the presence of AMP was measured in this study. The survival, migration, degranulation, respiratory burst, phagocytosis and intracellular calcium mobilisation of naïve and activated cells were tested to determine the effect of the bovine AMPs on these functions. As neutrophils are one of the first types of immune cell to respond to infection and inflammation, their key functions were measured after 1 hour exposure.

\subsubsection{Bovine $\alpha$-helical peptides induce cytotoxicity}

The cytotoxic effects of the different AMPs were measured by flow cytometry to determine the proportion of viable (annexin-V-negative/PI-negative), apoptotic (annexin-V-positive/PI-negative) and necrotic (PI-positive) cells. Analysis of the flow cytometry data showed that only BMAP-28-treated cells were significantly stained with PI, indicating that cell death was primarily the result of necrosis (Figure $4.1 \mathrm{~A}$ ). After stimulation with PMA, only BMAP-34 induced a significant drop in neutrophil viability, also via the induction of necrosis (Figure 4.1 B). A trend towards increased cytotoxicity in PMA-stimulated neutrophils was also observed for BMAP-28. The remaining peptides had no effect on naïve or activated neutrophil survival.

\subsubsection{BMAP-28 induces membrane permeabilisation}

BMAP-28 is known to be cytotoxic [127,224], driven by its ability to increase cell membrane permeability through pore formation [160]. To determine whether BMAP-28 and BMAP-34 were able to permeabilise the neutrophil membrane, peptide treated cells were incubated with the cell impermeable dye SYTOX Orange. As shown in Figure 4.1 C, neutrophils treated with PMA alone did not stain for SYTOX Orange indicating that the membrane was intact. Naïve neutrophils treated with BMAP-28 showed significant levels of intracellular staining. Activated cells were more susceptible 
to BMAP-28 permeabilisation, while BMAP-34 treatment only induced moderate staining of PMA-activated cells.

\subsubsection{Multiple bovine AMPs induce neutrophil migration}

Cathelicidin peptides LL-37 and PR-39 are reported to induce neutrophil migration at micromolar concentrations $[102,196]$. As these physically contrasting peptides were able to induce migration in their respective species, the bovine AMPs were each tested for their ability to induce bovine neutrophil migration. A modified Boyden chamber with a polycarbonate membrane containing $3 \mu \mathrm{m}$ pores was used to assess migration toward $5 \mu \mathrm{M}$ solutions of AMP. Bac1, linear Bac1, Bac5 and BMAP-28 each induced significant neutrophil migration (Figure 4.1 D). Weak migration was observed in response to BMAP-34, whereas indolicidin had no effect. 
A

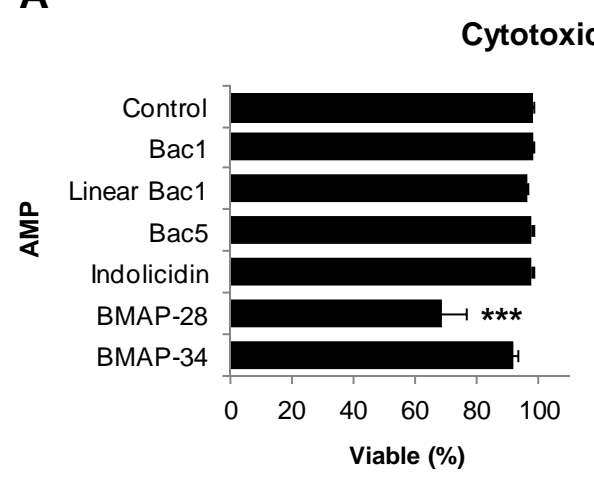

Cytotoxicity in naïve neutrophils

B
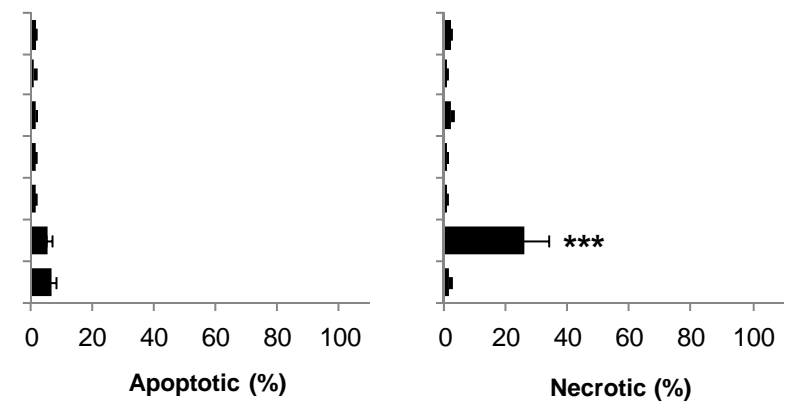

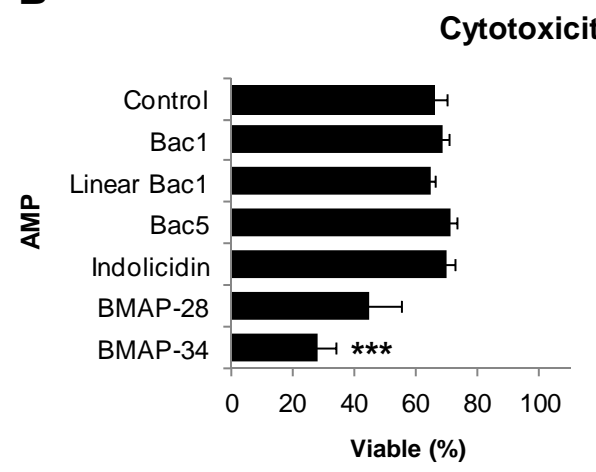

C

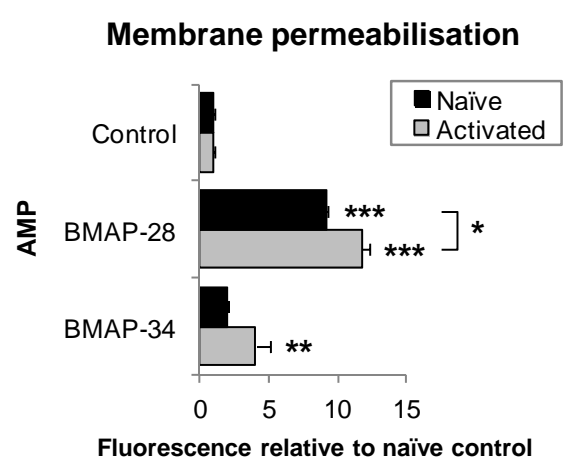

D

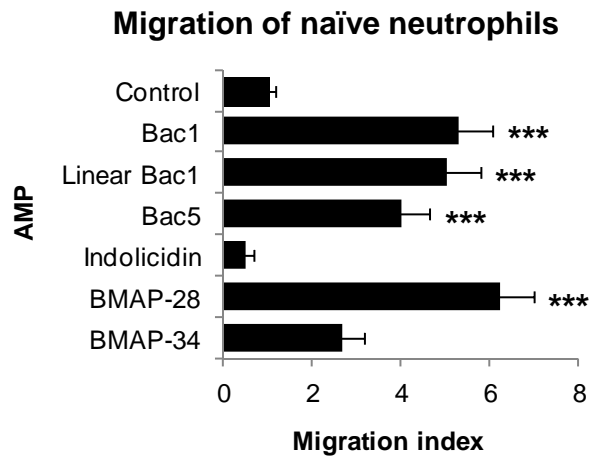

\section{Figure 4.1 Primary screen of neutrophil function 1}

Neutrophil functions were assessed after incubation in the presence of $5 \mu \mathrm{M}$ AMP for 1 hour. Cytotoxicity of AMPs toward (A) naïve cells and (B) cells activated with $100 \mathrm{ng} / \mathrm{ml}$ PMA was measured by flow cytometry. Annexin-V and PI were used to distinguish viable (annexin-V-negative/PI-negative), apoptotic (annexin-V-positive/PI-negative) and necrotic (PI-positive) cells. (C) Permeabilisation of neutrophils by BMAP-28 and BMAP-34 was measured using the cell-impermeant fluorescent dye SYTOX Orange. (D) Migration of naive neutrophils in response to $5 \mu \mathrm{M}$ AMP was assessed using a Boyden chamber. All data represent the mean \pm SEM for three independent experiments. Statistical significance was determined by one-way ANOVA with Tukey's post-test comparing treatments with control or Student's t-test comparing treatments. $* P<0.05$, ** $P<0.01$, *** $P<0.001$. 


\subsubsection{Degranulation is only affected by BMAP-28 treatment}

The process of neutrophil migration to inflammatory foci and activation commonly triggers neutrophil secretory granule release [42]. After 1 hour exposure to AMPs, granule contents released into the culture media from naïve and activated cells were measured by activity assays and immunoblotting. MPO activity was used as a measure of primary granule release. Western blot of CLD content was used as a measure of large granule release. Alkaline phosphatase activity was used as a measure of secretory vesicle release. Degranulation in response to the peptides was only observed following BMAP-28 treatment where both naïve and activated cells released significant amounts of secretory vesicles and large granules (Figure 4.2 A, B).

\subsubsection{Bovine AMPs have different effects on respiratory burst}

Respiratory burst is one of the methods by which neutrophils orchestrate bacterial killing. It is characterised by the activation of NADPH oxidase leading to production of superoxide radical and ROS [48]. Extracellular superoxide production by naïve and activated neutrophils exposed to AMP was measured via the reduction of WST-1 to a yellow formazan salt. As shown in Figure 4.2 C, a trend for increasing stimulation by Bac5 was observed in naïve neutrophils. Linear Bac1 and indolicidin induced significant superoxide production in naïve neutrophils. BMAP-28 and BMAP-34 suppressed basal levels of respiratory burst in naïve cells and also suppressed respiratory burst in activated cells (Figure 4.2 D).

\subsubsection{Bac5 enhances phagocytosis}

Neutrophils are phagocytes. To investigate whether the AMPs affected phagocytosis, AMP-treated neutrophils were incubated with opsonised fluorescent beads and analysed by flow cytometry to identify fluorescence-positive cells. Bac5 induced a significant increase in neutrophil phagocytosis compared to untreated controls (Figure 4.2 E, F). None of the other peptides significantly altered bead uptake. 
A

Degranulation by naïve neutrophils

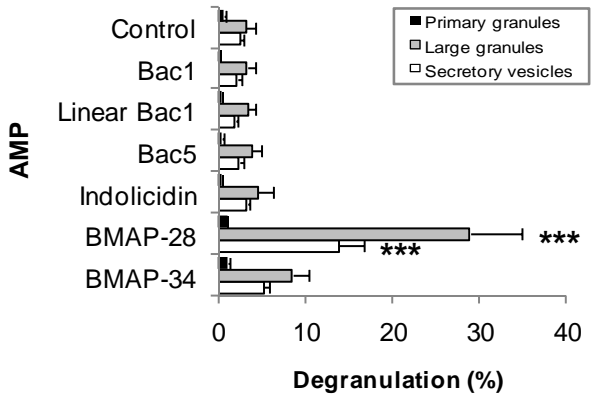

C

Respiratory burst by naïve neutrophils

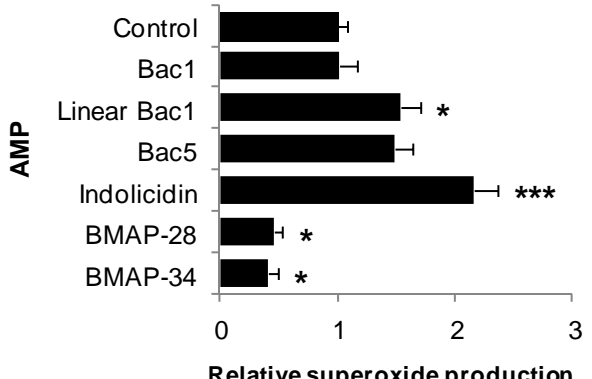

E

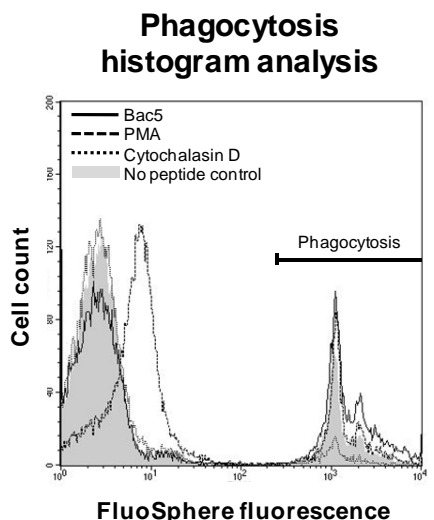

B

Degranulation by activated neutrophils

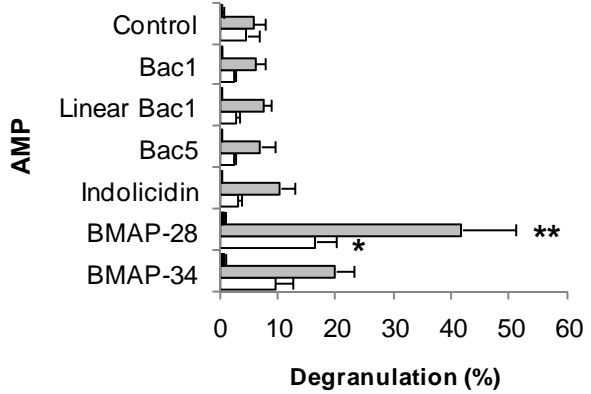

D

Respiratory burst by activated neutrophils

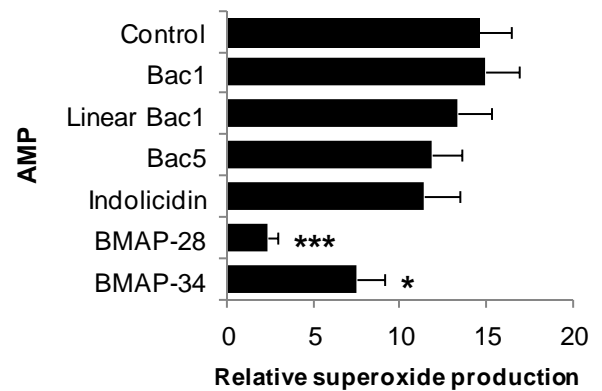

$\mathbf{F}$

Phagocytosis by naïve neutrophils

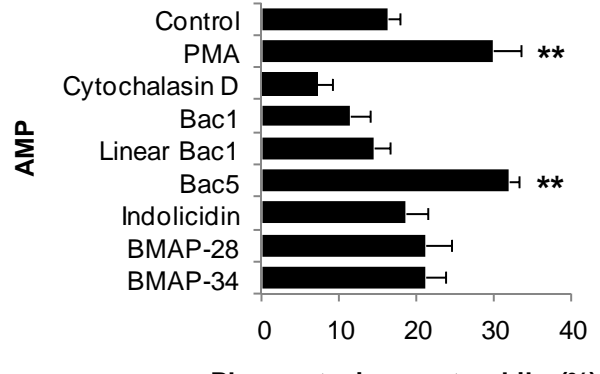

Phagocytosing neutrophils (\%)

\section{Figure 4.2 Primary screen of neutrophil function 2}

Neutrophil functions were assessed after incubation in the presence of $5 \mu \mathrm{M}$ AMP for 1 hour. Degranulation was assessed by measuring the release of protein markers of primary granules, large granules, and secretory vesicles in (A) naïve cells and (B) cells activated with $100 \mathrm{ng} / \mathrm{ml} \mathrm{PMA,} \mathrm{after}$ incubation with AMP. Respiratory burst was measured in (C) naïve cells and (D) cells activated with $100 \mathrm{ng} / \mathrm{ml}$ PMA by reduction of WST-1. Phagocytosis was assessed by the uptake of opsonised fluorescent beads using flow cytometry. (E) Representative histogram analysis of phagocytosis. (F) Combined phagocytosis histogram data. All data represent the mean \pm SEM for three independent experiments. Statistical significance was determined by one-way ANOVA with Tukey's post-test comparing treatments with control. $* P<0.05, * * P<0.01, * * * P<0.001$. 


\subsubsection{Intracellular calcium mobilisation is not affected by AMP treatment}

Neutrophil innate immune functions are commonly induced by changes in intracellular calcium concentrations [225,226]. To measure intracellular calcium mobilisation, neutrophils were loaded with the fluorescent calcium ion indicator Fluo-4 AM. Naïve or activated neutrophils were then treated with AMP and changes in fluorescence were monitored over 1 hour. Treatment with zymosan-activated serum served as a positive control which induced a characteristic transitory increase in fluorescence, indicating the cells were correctly loaded with Fluo-4 AM. None of the peptides affected intracellular calcium flux in either naïve (Figure $4.3 \mathrm{~A}$ ) or activated (Figure $4.3 \mathrm{~B}$ ) cells except for BMAP-28. BMAP-28 induced a gradual increase in fluorescence in naïve and activated cells, which was similar to that induced by $10 \mu \mathrm{M}$ calcium ionophore A23187. This indicated a steady flux of calcium, and is consistent with cell permeabilisation induced by this peptide. These results are also consistent with the fact that activation with PMA does not induce calcium flux in bovine (or human) neutrophils [210]. It is also interesting to note that cytotoxicity induced in activated cells by BMAP-34 is not accompanied by changes in intracellular calcium and indicates BMAP-34 induces a controlled mode of cell death. 
A
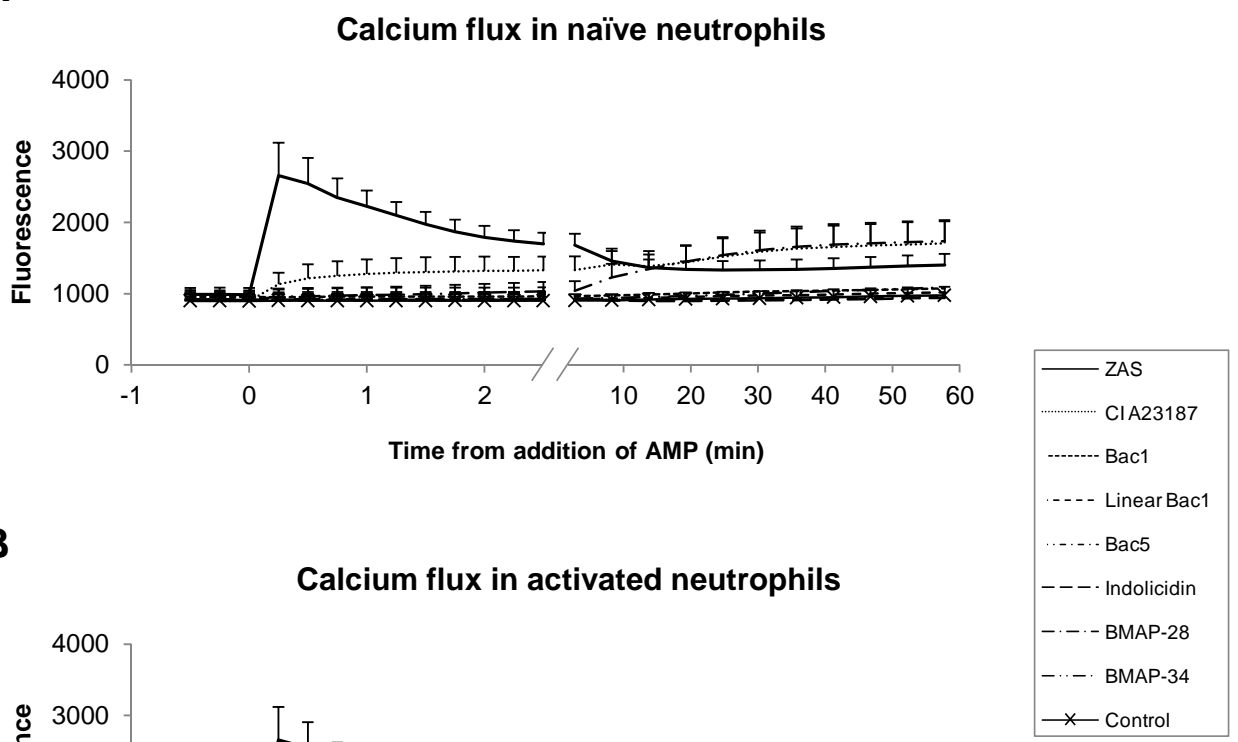

B

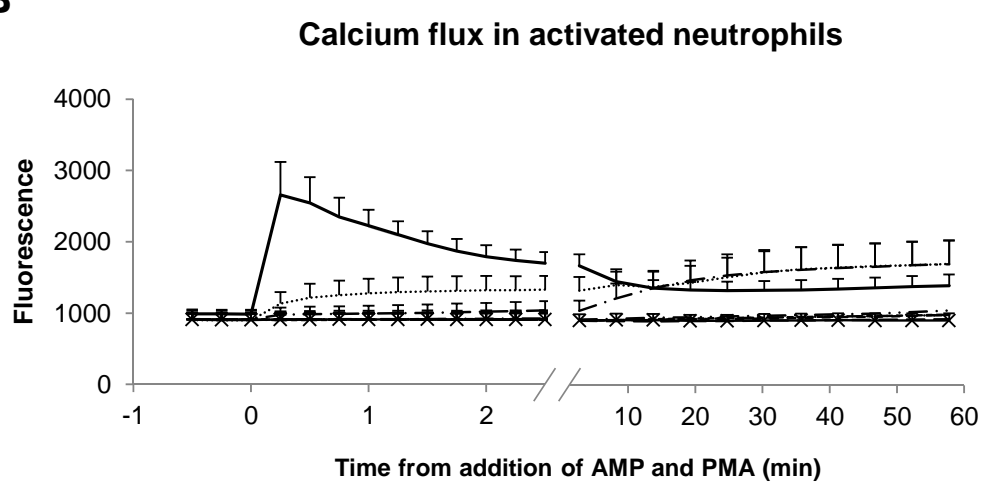

$\times$ Control

\section{Figure 4.3 Primary screen of neutrophil function 3}

Intracellular calcium mobilisation of (A) naïve cells and (B) cells activated with $100 \mathrm{ng} / \mathrm{ml}$ PMA was monitored in the presence of AMP over 1 hour. Neutrophils were loaded with Fluo-4 AM before exposure to treatments. BMAP-28-induced calcium mobilisation was significant (arbitrary fluorescence units, $P<0.001)$ after 10 minutes in both naïve and PMA-activated neutrophils. Note the change in time scale indicated by the break. Data represent the mean \pm SEM for at least three independent experiments performed in triplicate. Statistical significance was determined by one-way ANOVA with Tukey's posttest comparing treatments with control. CI A23187, calcium ionophore A23187; ZAS, zymosan-activated serum. 


\subsubsection{Summary of the primary screen analysis of neutrophil function}

A summary of the results from the primary screen is provided in Table 4.2 and Table 4.3. Due to the ability to induce or inhibit key neutrophil functions in the absence of cytotoxic effects (with the caveat of BMAP-34 only inducing cell death in PMAactivated neutrophils), linear Bac1, Bac5 and BMAP-34 were selected for further analysis in dose-response assays. BMAP-28 was excluded because significant cell death was induced in naïve and activated cells, suggesting the pro-inflammatory effects were primarily due to non-specific cell death. Indolicidin and the $\beta$-turn form of Bac1 were excluded as they were only able to respectively induce migration or respiratory burst. However, the selective nature of these two peptides is worthy of future investigation.

Table 4.2 Primary screen summary 1

\begin{tabular}{|c|c|c|c|c|c|}
\hline \multirow[b]{3}{*}{ Peptide } & \multicolumn{5}{|c|}{ Neutrophil response } \\
\hline & \multicolumn{2}{|c|}{ Cell death } & \multirow{2}{*}{$\begin{array}{c}\text { Migration } \\
\text { Naïve }\end{array}$} & \multicolumn{2}{|c|}{ Granule release } \\
\hline & Naïve & Activated & & Naïve & Activated \\
\hline Bac1 & & & + & & \\
\hline Linear Bac1 & & & + & & \\
\hline Bac5 & & & + & & \\
\hline Indolicidin & & & & & \\
\hline BMAP-28 & + & & + & + & + \\
\hline BMAP-34 & & + & & & \\
\hline
\end{tabular}

(+) indicates induction or enhancement; (-) indicates inhibition

Table 4.3 Primary screen summary 2

\begin{tabular}{|c|c|c|c|c|c|}
\hline \multirow[b]{3}{*}{ Peptide } & \multicolumn{5}{|c|}{ Neutrophil response } \\
\hline & \multicolumn{2}{|c|}{ Respiratory burst } & \multirow{2}{*}{$\begin{array}{c}\text { Phagocytosis } \\
\text { Naïve }\end{array}$} & \multicolumn{2}{|c|}{ Calcium flux } \\
\hline & Naïve & Activated & & Naïve & Activated \\
\hline \multicolumn{6}{|l|}{ Bac1 } \\
\hline Linear Bac1 & + & & & & \\
\hline Bac5 & & & + & & \\
\hline Indolicidin & + & & & & \\
\hline BMAP-28 & - & - & & + & + \\
\hline BMAP-34 & - & - & & & \\
\hline
\end{tabular}

(+) indicates induction or enhancement; (-) indicates inhibition 


\subsubsection{Dose-response analysis of neutrophil function}

In this section the functional assays were adjusted to a dose-response format, and where appropriate, optimised to better mimic the in vivo conditions neutrophils encounter during inflammation. Starting at a maximum concentration of $10 \mu \mathrm{M}$, a two-fold titration of each peptide was tested to determine dose-response effects. For migration assays a ten-fold titration was tested.

\subsubsection{Cytotoxicity}

To determine the effect of bovine cathelicidins on neutrophil survival, naïve and activated neutrophils were incubated with the selected AMPs for 1 hour. Because minimal cytotoxicity was observed after 1 hour, cytotoxicity was also measured after 6 hours, before the time at which neutrophils undergo spontaneous apoptosis [55]. Pacific Blue-conjugated annexin-V was used to distinguish apoptosis. Due to the large number of samples to be analysed by flow cytometry, a fixable necrotic cell dye (LIVE/DEAD Fixable Red Dead Cell Stain) was used, as propidium iodide has the propensity to leach out of dead cells that are not analysed soon after staining.

Consistent with the results of the preliminary assays, the AMPs had little effect on naïve neutrophil viability within the first hour except for BMAP-34 which induced significant cell death at $10 \mu \mathrm{M}$, primarily by necrosis (Figure $4.4 \mathrm{~A}$ ). After 6 hours, Bac5 treatment increased cell viability over the full concentration range whereas linear Bac1 and BMAP-34 each improved cell survival at lower concentrations (Figure 4.4 B).

A significant loss of cells from analysis occurred after neutrophils were treated with PMA. It is likely that activation caused a majority of cells to tightly adhere to culture plates despite the plates being treated to prevent adherence, and unintentional damage to cells is likely to have ensued during the staining and wash procedures. Consequently, the results probably do not accurately reflect the true proportions of viable, apoptotic and necrotic cells. However, in the activated neutrophils that could be analysed, all three AMPs showed a trend toward inducing necrotic cell death as the concentration increased (BMAP-34 > Bac5 > linear Bac1) (Figure 4.5 A, B). 
A

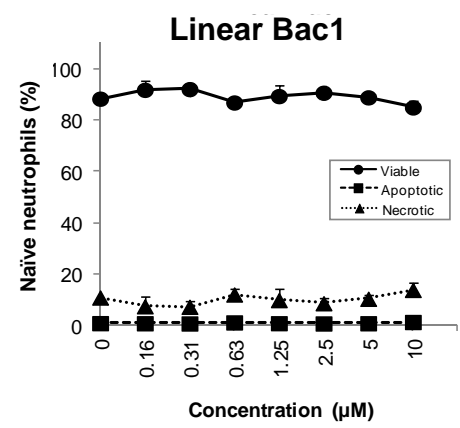

B

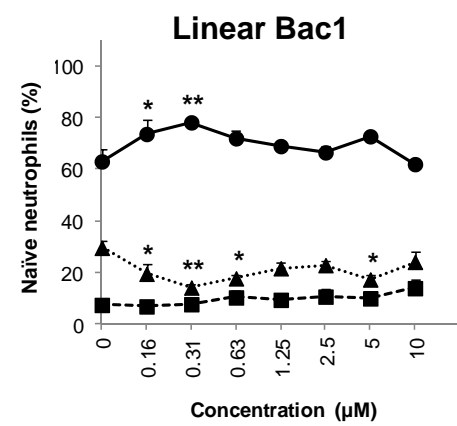

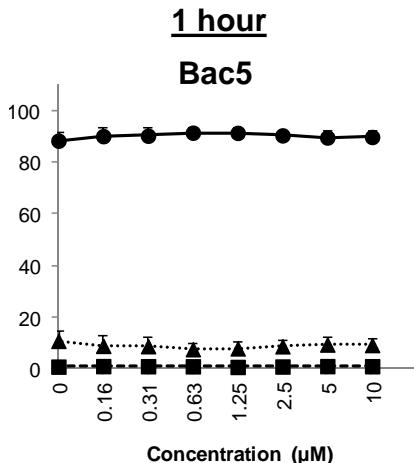

$\underline{6 \text { hours }}$
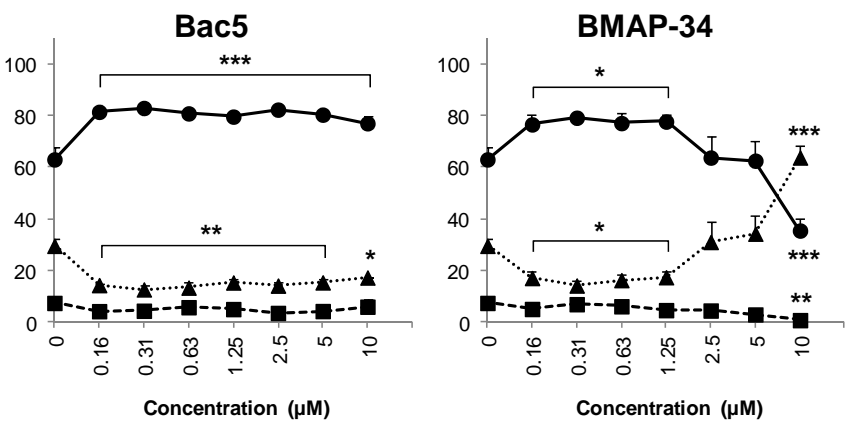

Figure 4.4 Survival of naïve neutrophils after linear Bac1, Bac5 or BMAP-34 treatment

Survival of naïve cells after (A) 1 hour or (B) 6 hours of AMP treatment was measured by flow cytometry. Pacific Blue-annexin-V and LIVE/DEAD Fixable Red Dead Cell Stain were used to distinguish viable (annexin-V-negative, LIVE/DEAD-negative), apoptotic (annexin-V-positive, LIVE/DEAD-negative) and necrotic (LIVE/DEAD-positive) cells. The cells were fixed with $4 \%$ (w/v) paraformaldehyde in PBS, washed, and resuspended in sheath fluid before analysis. Data represent the mean \pm SEM for three independent experiments performed in duplicate. Statistical significance was determined by one-way ANOVA with Fisher's post hoc test comparing AMP treatments with untreated controls $(0 \mu \mathrm{M}$ AMP). $* P<0.05, * * P<0.01, * * * P<0.001$. 
A
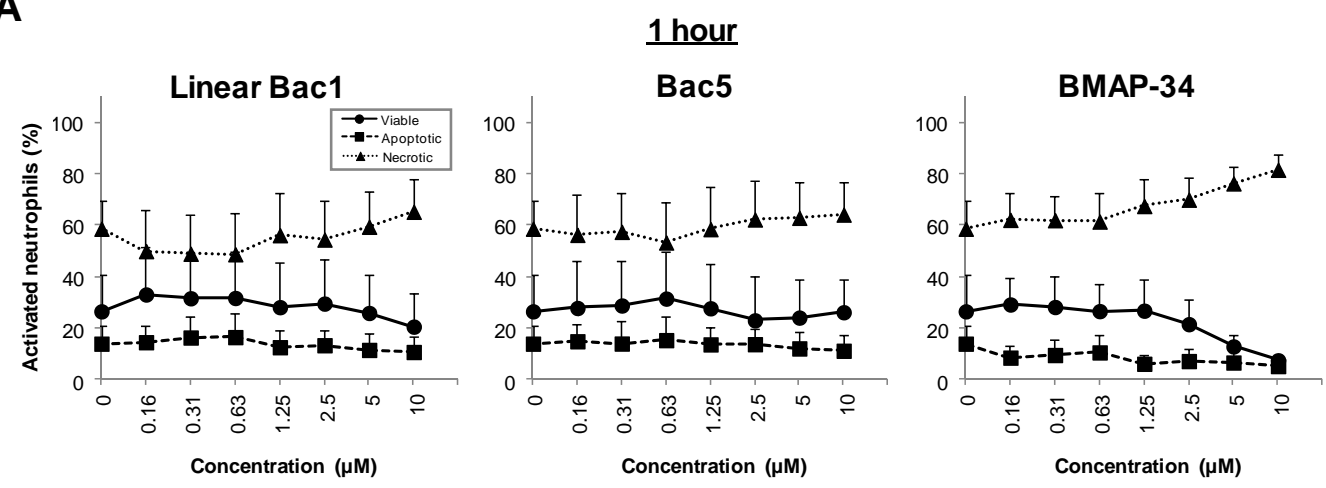

B
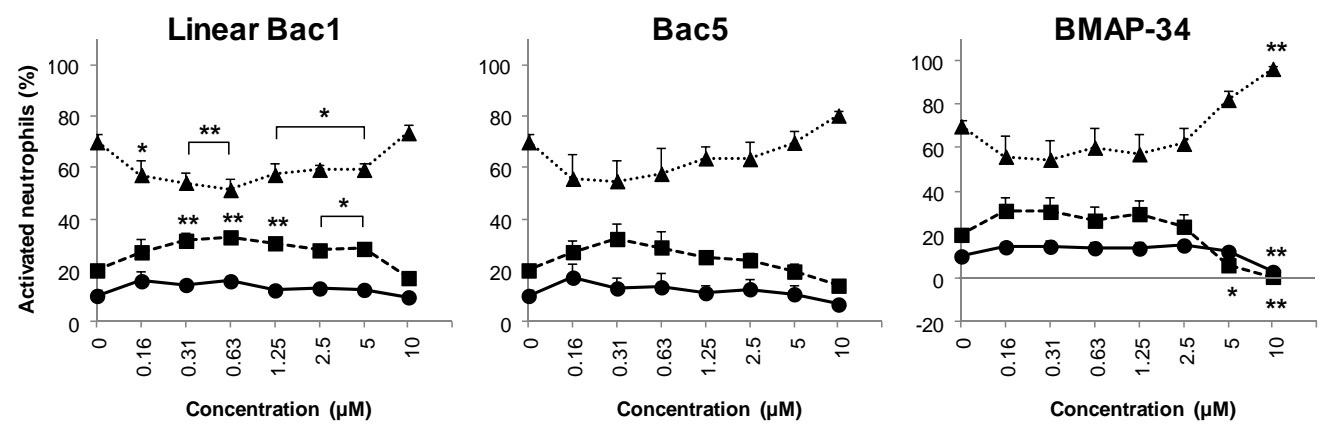

Figure 4.5 Survival of activated neutrophils after linear Bac1, Bac5 or BMAP-34 treatment

Survival of cells activated with $100 \mathrm{ng} / \mathrm{ml}$ PMA after (A) 1 hour or (B) 6 hours of AMP treatment was measured by flow cytometry. Pacific Blue-annexin-V and LIVE/DEAD Fixable Red Dead Cell Stain were used to distinguish viable (annexin-V-negative, LIVE/DEAD-negative), apoptotic (annexin-Vpositive, LIVE/DEAD-negative) and necrotic (LIVE/DEAD-positive) cells. The cells were fixed with $4 \%$ (w/v) paraformaldehyde in PBS, washed, and resuspended in sheath fluid before analysis. Data represent the mean \pm SEM for three independent experiments performed in duplicate. Statistical significance was determined by one-way ANOVA with Fisher's post hoc test comparing AMP treatments with untreated controls $(0 \mu \mathrm{M}$ AMP). $* P<0.05$, ** $P<0.01$, *** $P<0.001$. 


\subsubsection{Migration}

Neutrophil chemoattractants commonly induce their effects at nanomolar concentrations. Therefore the ability of linear Bac1, Bac5 or BMAP-34 to induce the migration of naïve neutrophils was assessed at ten-fold concentrations between $10 \mu \mathrm{M}$ and $1 \mathrm{nM}$. Recombinant human IL-8 was used as a positive control at $5 \mathrm{ng} / \mathrm{ml}$. The polycarbonate membranes were coated in Type I collagen to better mimic tissues through which neutrophils migrate in response to inflammatory signals. As shown in Figure 4.6, all of the AMPs exhibited notably different migration profiles as the AMP concentration increased. Linear Bac1 was able to induce significant migration at $1 \mathrm{nM}$ which decreased at higher concentrations. The Bac5 migration index increased with concentration to a maximum comparable to the positive control $(5 \mathrm{ng} / \mathrm{ml}$ recombinant human interleukin-8, migration index $=6.84 \pm 2.82$ ). The maximal response to BMAP-34 peaked at $100 \mathrm{nM}$ then dropped significantly. This was consistent with the decrease in cell survival observed at higher concentrations of BMAP-34 (see Figure 4.4 A).
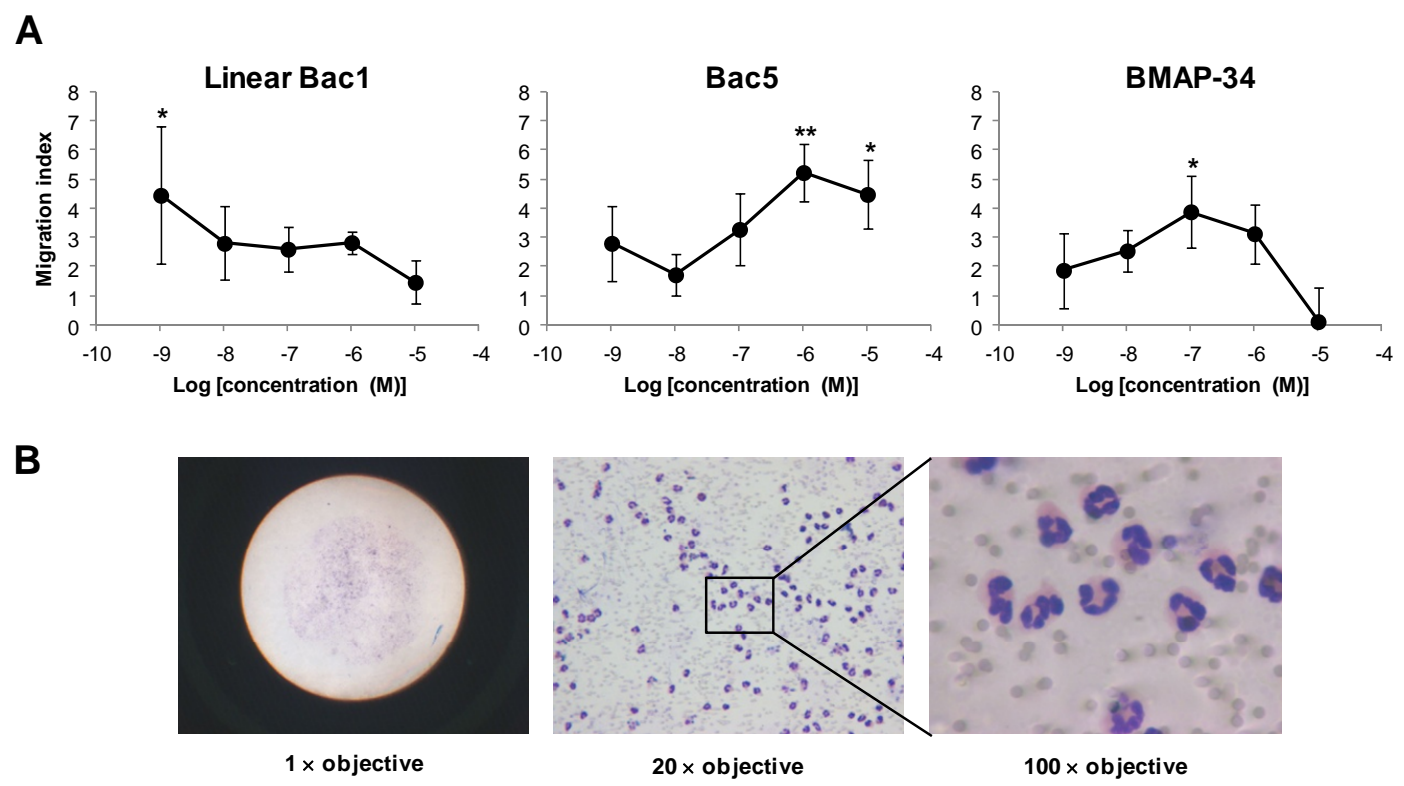

\section{Figure 4.6 Neutrophil migration induced by linear Bac1, Bac5 or BMAP-34}

(A) Neutrophil migration was assessed using a Boyden chamber. Polycarbonate membranes were coated in collagen (Type I) and migration proceeded for 1 hour. (B) From left to right: representative Boyden chamber well at $1 \times$ objective, $20 \times$ objective and $100 \times$ objective (oil immersion). Data represent the mean \pm SEM for two independent experiments (linear Bac1) or four independent experiments (Bac5, BMAP-34) performed in duplicate. Statistical significance was determined by one-way ANOVA with Tukey's post-test comparing AMP treatments with untreated controls. $* P<0.05$, $* * P<0.01$. 


\subsubsection{Degranulation}

Significant degranulation for linear Bac1, Bac5 and BMAP-34 was not observed in the primary screen assays, therefore the dose-response assays were extended out to 6 hours. A trend toward increasing primary granule release was observed after 6 hours treatment with linear Bac1, which peaked at $1.25 \mu \mathrm{M}$ (Figure 4.7 A). Bac5 and BMAP-34 were unable to significantly alter primary granule release in naïve neutrophils. Activated neutrophils did not release as much MPO as naïve neutrophils. Even when treated with peptide the response remained suppressed, with linear Bac1 and BMAP-34 inducing minor increases at the lowest concentration tested. Bac5 produced a similar increase at $2.5 \mu \mathrm{M}$ only. Overall, primary granule release was minor, with less than $10 \%$ of the total cellular MPO released at any time.

The secondary granules of bovine neutrophils contain humoral mediators of the innate immune response which are bacteriostatic and bactericidal in nature. These mediators include lysozyme, lactoferrin and TCII (vitamin B12 binding protein) [227]. Therefore, in the dose-response assays, secondary granule release was measured instead of secretory vesicle release. Western blot was used to measure TCII as an indicator of secondary granule release (Figure $4.7 \mathrm{~B}$ ) and dot blot was used to measure CLD as an indicator of large granule release (Figure $4.7 \mathrm{C}$ ). All three peptides appeared to suppress secondary granule release by naive neutrophils whereas Bac5 dose-dependently increased secondary granule release in activated cells (Figure $4.7 \mathrm{~B}$ ). Linear Bac1 also appeared to enhance secondary granule release in activated cells but not to a significant degree.

Neither linear Bac1 nor Bac5 had a significant effect on large granule release by naive or activated cells (Figure $4.7 \mathrm{C}$ ). However, naïve neutrophils treated with BMAP-34 exhibited a dose-dependent increase in large granule release that then dropped away at higher concentrations. A similar effect was observed in activated cells, where the amount of CLD released reached a maximum at $0.63 \mu \mathrm{M}$ BMAP-34, which dropped below background levels at higher concentrations. 
A

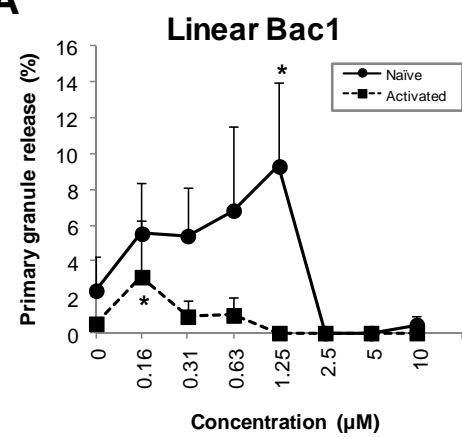

B

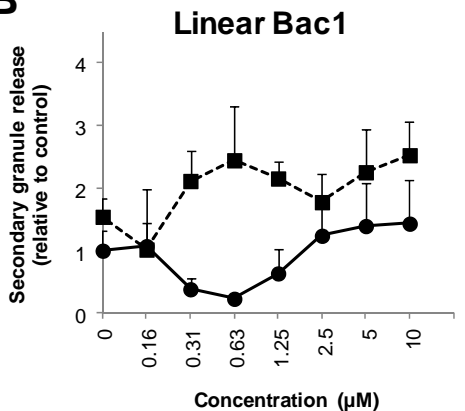

C

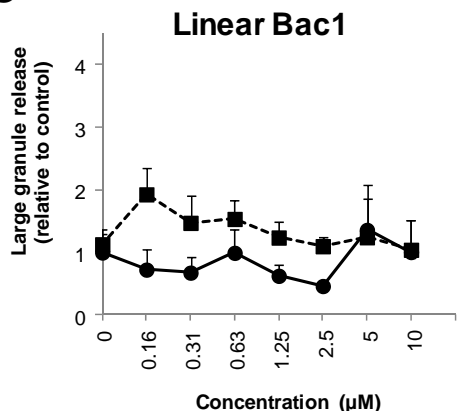

Bac5

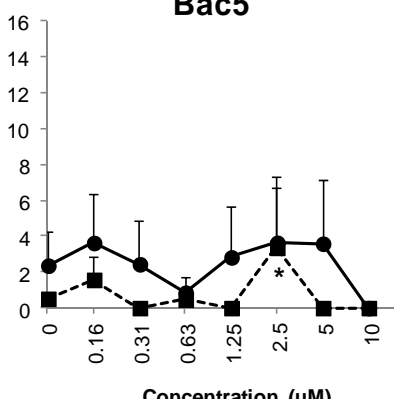

Concentration $(\mu \mathrm{M})$

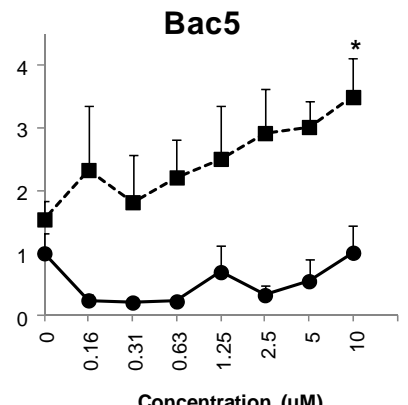

Bac5

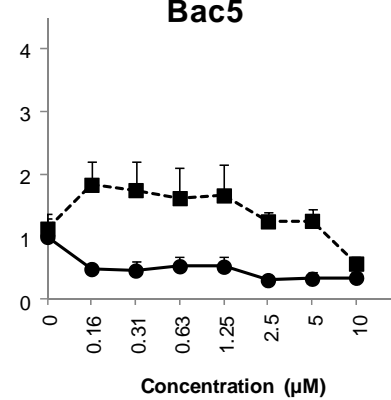

BMAP-34

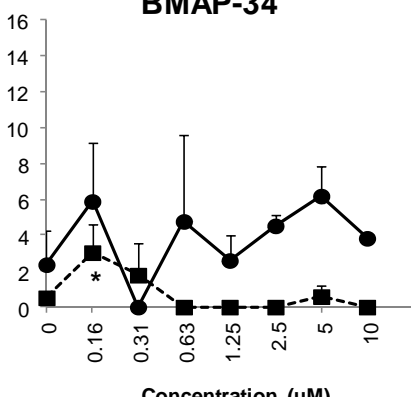

BMAP-34

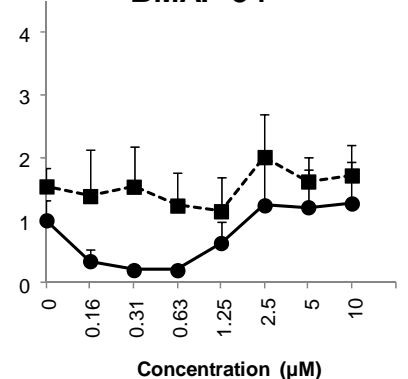

BMAP-34

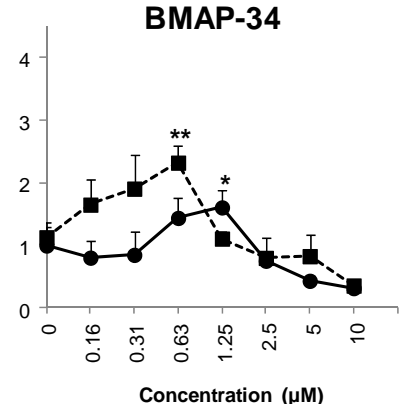

Figure 4.7 Neutrophil granule release after 6 hours of linear Bac1, Bac5 or BMAP-34 treatment

Naïve cells and cells activated with $100 \mathrm{ng} / \mathrm{ml}$ PMA were treated with AMP for 6 hours. MPO activity in neutrophil culture supernatants was used as an indicator of $(\mathbf{A})$ primary granule release. The percentage of primary granule release was determined from the total MPO activity of an equivalent number of cells. Data represent the mean \pm SEM for two independent experiments performed in triplicate. Western blot and dot blot of the granule markers TCII and CLD in neutrophil culture supernatants were used as an indicator of $(\mathbf{B})$ secondary and $(\mathbf{C})$ large granule release, respectively. Data represent the mean \pm SEM for three independent experiments performed in triplicate. Statistical significance was determined by oneway ANOVA with Fisher's post hoc test comparing AMP treatments with untreated controls $(0 \mu \mathrm{M}$ AMP). $* P<0.05, * * P<0.01$. 


\subsubsection{Respiratory burst}

Measurement of superoxide production induced by linear Bac1, Bac5 and BMAP-34 gave similar results to the screening assays. As shown in Figure $4.8 \mathrm{~A}$, linear Bac1 and Bac5 induced a dose-dependent increase in superoxide production in naïve neutrophils. The approximate $\mathrm{EC}_{50}$ values were $1.66 \pm 0.24 \mu \mathrm{M}$ and $0.39 \pm 0.17 \mu \mathrm{M}$, respectively. As seen in the primary screen assay, BMAP-34 inhibited superoxide production compared to untreated controls. The strong production of superoxide elicited by PMA (PMA-treated controls $=16.63 \pm 1.70)$ was not affected by either linear Bac1 or BMAP-34 (Figure 4.8 B). However, $10 \mu \mathrm{M}$ Bac5 was able to significantly inhibit PMAinduced superoxide production.

A Naïve neutrophils
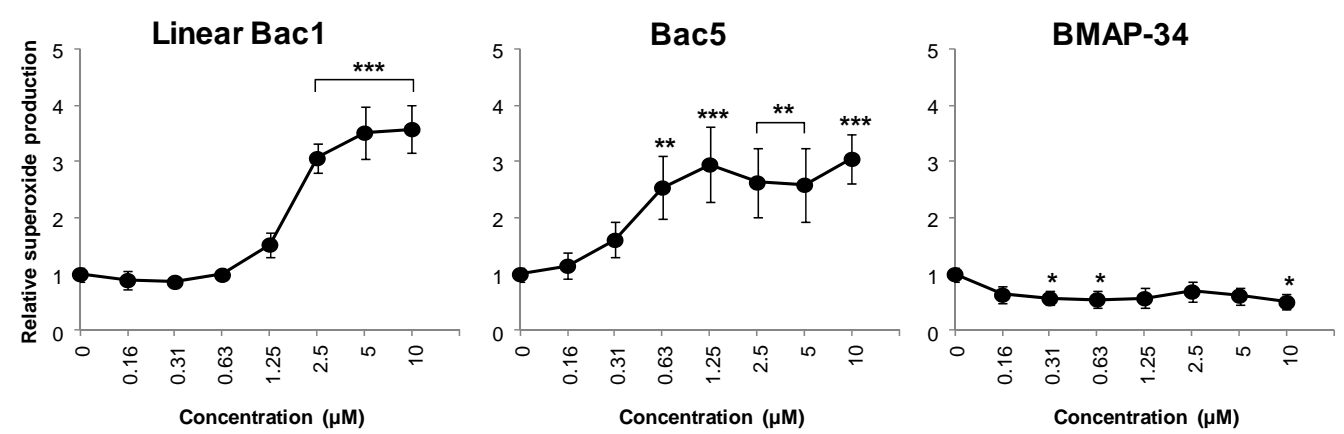

B Activated neutrophils
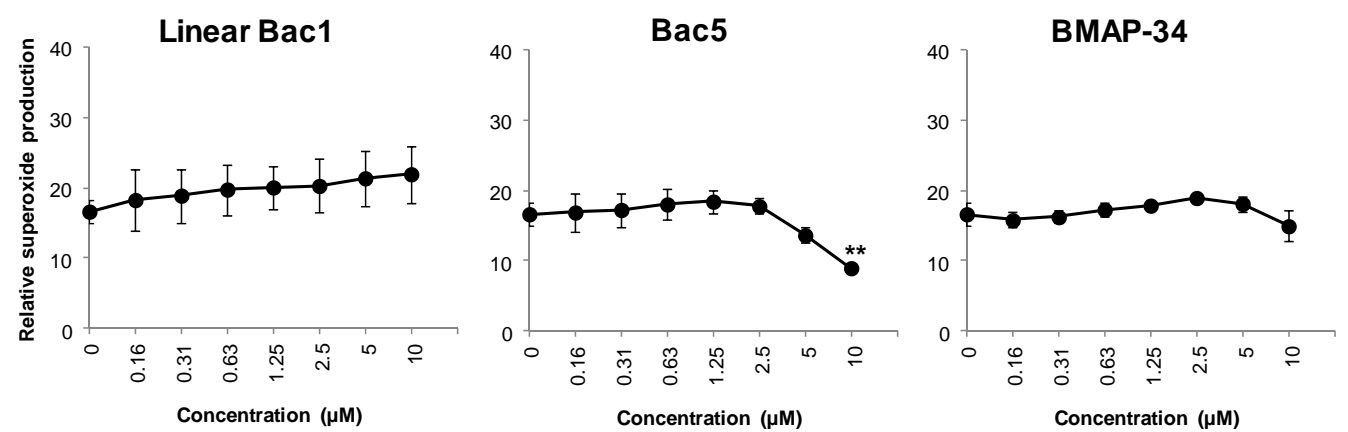

Figure 4.8 Neutrophil respiratory burst after linear Bac1, Bac5 or BMAP-34 treatment

Extracellular superoxide production was measured using the colourimetric dye WST-1. Absorbance was measured after 1 hour exposure to AMP for (A) naïve cells and (B) cells activated with $100 \mathrm{ng} / \mathrm{ml} \mathrm{PMA.}$ Data represent the mean WST-1 reduction relative to naïve controls \pm SEM for three independent experiments performed in triplicate. Statistical significance was determined by one-way ANOVA with Fisher's post hoc test comparing AMP treatments with untreated controls $(0 \mu \mathrm{M} \mathrm{AMP})$. $* P<0.05$, $* * P<0.01, * * * P<0.001$. 


\subsubsection{Phagocytosis}

Assays of neutrophil phagocytosis in the presence of linear Bac1, Bac5 and BMAP-34 were modified so that the cells were not pretreated with AMP. Instead, peptide was added along with the opsonised beads. Additionally, the opsonised beads were washed in media prior to their addition to the assay to remove traces of activated serum. Cytochalasin B inhibits phagocytosis by interfering with actin filament formation and was used as a negative control of phagocytosis in these assays, while PMA was used as a positive control. All three peptides enhanced phagocytosis in a dose-dependent manner (Figure 4.9).

\subsubsection{Intracellular calcium mobilisation}

The assays for calcium mobilisation remained unchanged from the primary screen assays. None of the AMPs induced a change in fluorescence in either naïve (Figure $4.10 \mathrm{~A}$ ) or activated (Figure $4.10 \mathrm{~B}$ ) neutrophils at any of the concentrations tested. The same result was observed after 1 hour (Figure $4.10 \mathrm{C}, \mathrm{D}$ ). These data indicated calcium mobilisation within neutrophils remained unchanged throughout treatment. 
A
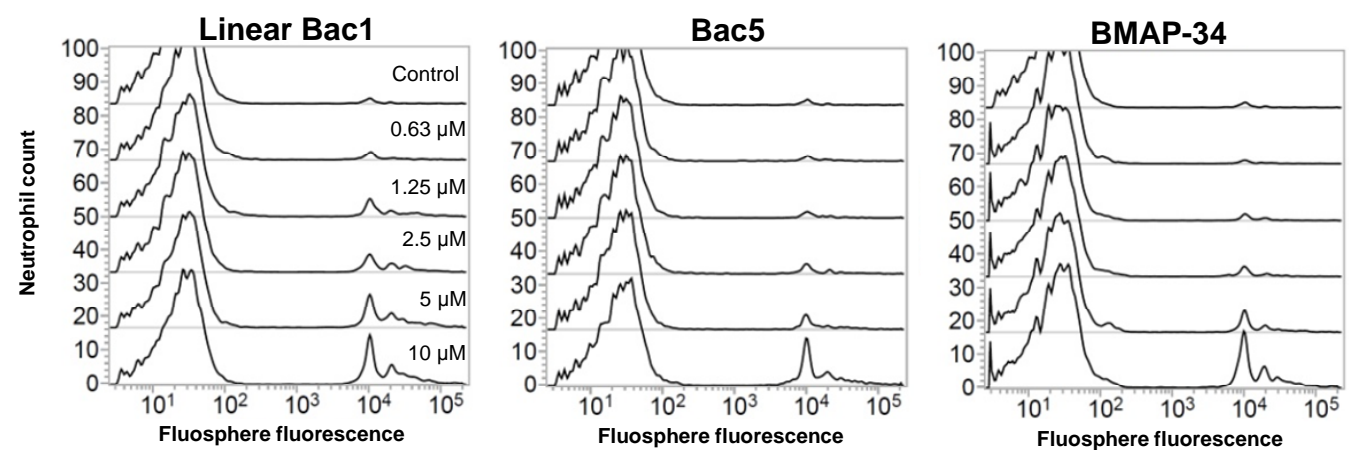

B
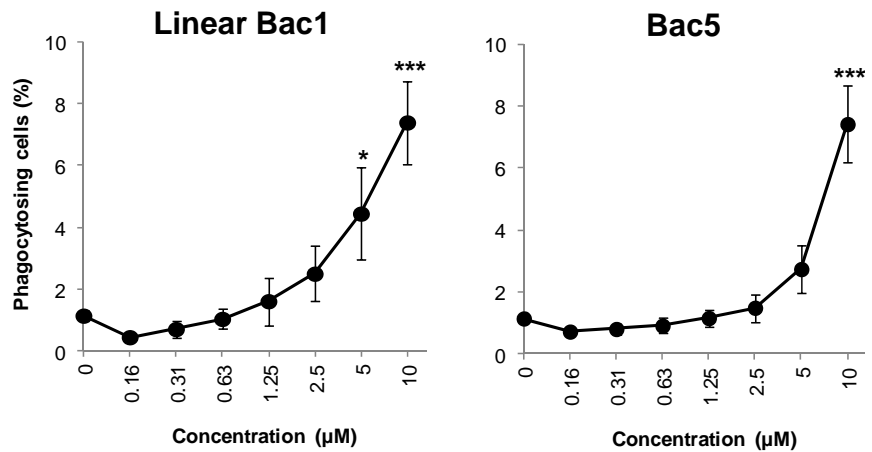

BMAP-34
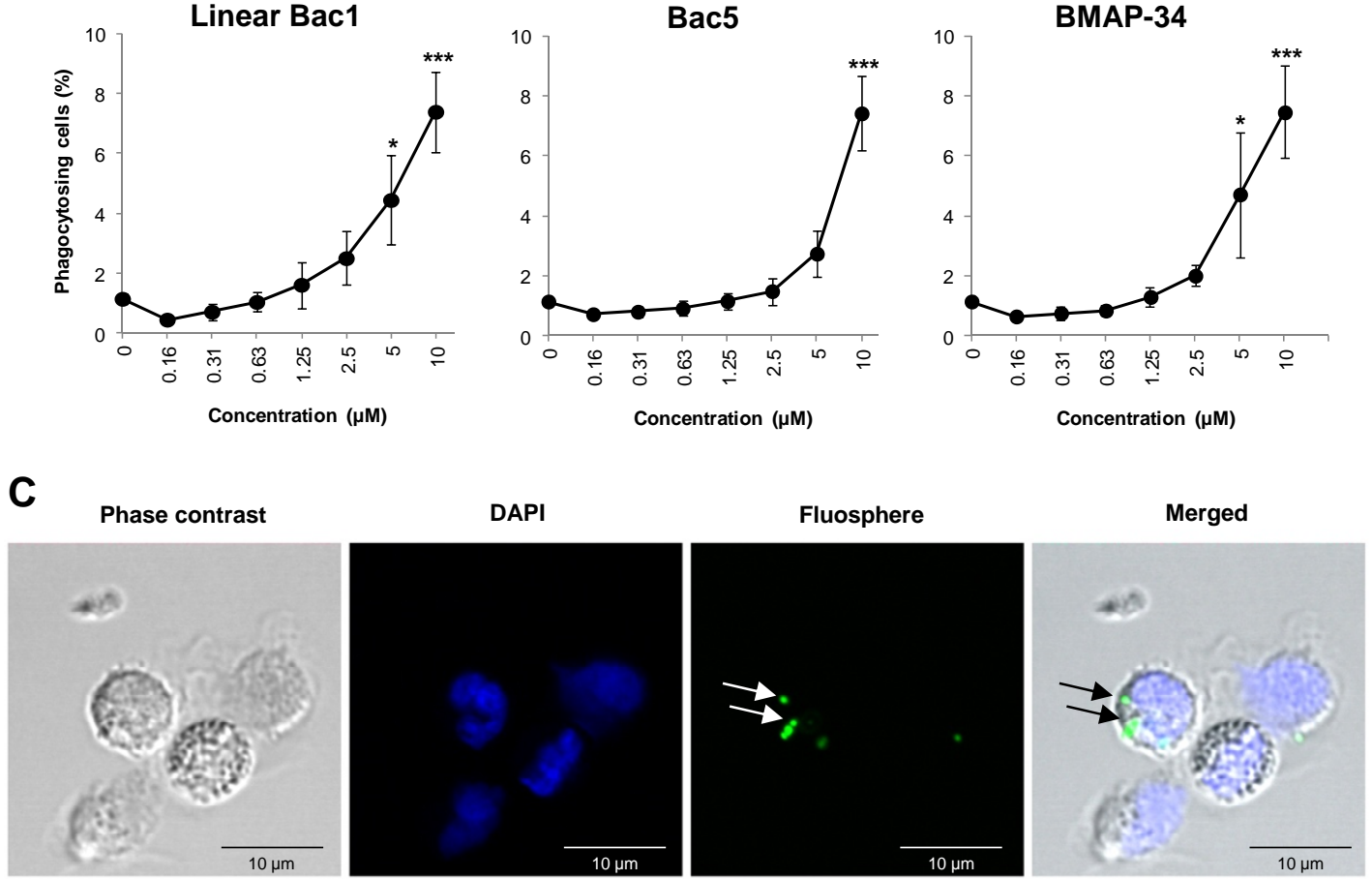

Figure 4.9 Phagocytosis during linear Bac1, Bac5 or BMAP-34 treatment

Neutrophils were incubated in the presence of AMP and opsonised FluoSpheres for 1 hour before analysis by flow cytometry. (A) Representative histogram analysis. (B) Proportion of cells that had completely phagocytosed beads during AMP treatment. (C) Representative fluorescence photomicrograph of a phagocytosing neutrophil showing internal FluoSpheres (arrows). Control values: $10 \mu \mathrm{M}$ cytochalasin B, $1.66 \pm 0.38 \% ; 100 \mathrm{ng} / \mathrm{ml}$ PMA, $6.72 \pm 1.49 \%, P<0.05$. Data represent the mean \pm SEM for three independent experiments performed in duplicate. Statistical significance was determined by one-way ANOVA with Fisher's post hoc test comparing AMP treatments with untreated controls $(1.15 \pm 0.18 \%)$.

$* P<0.05, * * * P<0.001$. 
A
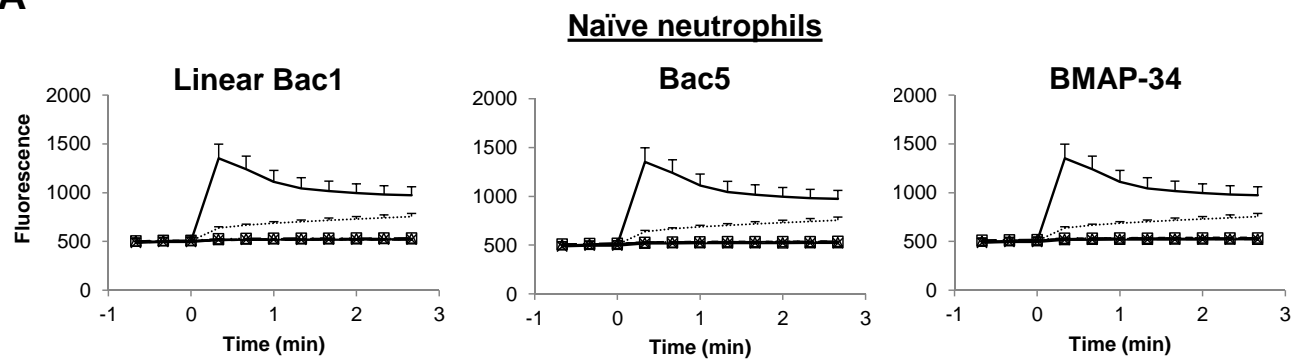

B

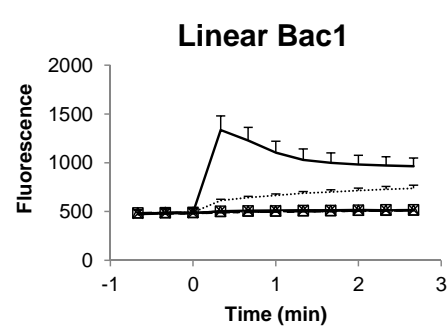

Activated neutrophils
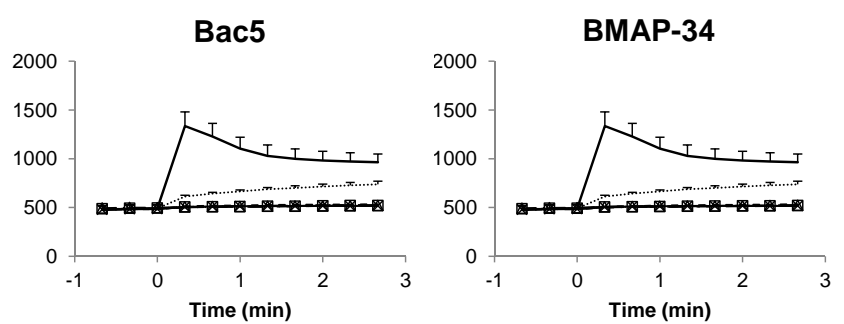

C

Intracellular calcium in naïve neutrophils after 1 hour treatment

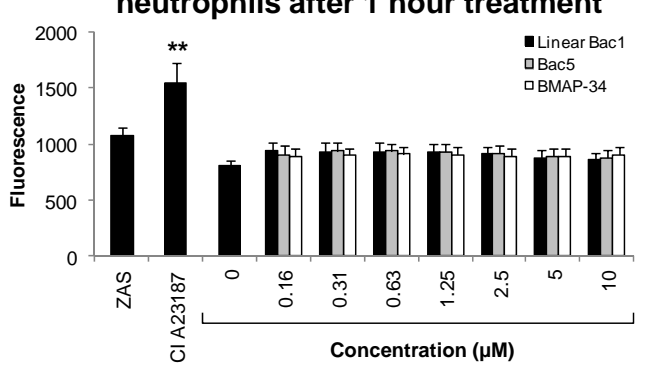

D

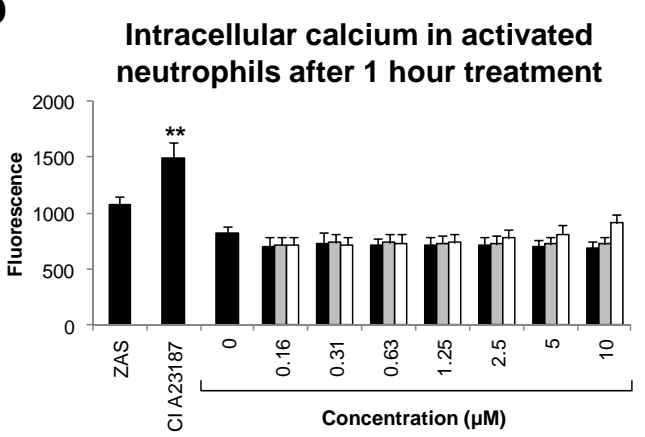

Figure 4.10 Calcium mobilisation during linear Bac1, Bac5 or BMAP-34 treatment

Neutrophils were loaded with Fluo-4 AM and changes in intracellular calcium of (A) naïve cells and (B) cells activated with $100 \mathrm{ng} / \mathrm{ml}$ PMA were monitored in the presence of AMP for 3 minutes. AMP alone or AMP and PMA were added at 0 minutes. After 1 hour exposure the fluorescence of Fluo- 4 AM was also measured for (C) naïve cells and (D) cells activated with $100 \mathrm{ng} / \mathrm{ml}$ PMA. Data represent the mean \pm SEM for three independent experiments performed in triplicate. Statistical significance was determined by one-way ANOVA with Fisher's post hoc test comparing AMP treatments with untreated controls (0 $\mu$ M AMP). ** $P<0.01$. CI A23187, calcium ionophore A23187; ZAS, zymosan-activated serum. 


\subsubsection{Summary of the dose-response analysis of neutrophil function}

A summary of the dose-response analysis is provided in Table 4.4 and Table 4.5. Differences in relation to the primary screen assays generally occur due to modification of the experimental approach. However, it should be noted that variation in results between the primary screen analysis and the dose-response analysis could result in part from seasonal variation in cattle immune responses. This is a well reported phenomenon in several species including humans [228-230]. This is particularly relevant considering the primary screen data were collected in autumn and winter, and the dose-response data were collected in spring and summer.

Table 4.4 Dose-response summary 1

\begin{tabular}{|c|c|c|c|c|c|c|c|c|}
\hline \multirow[b]{4}{*}{ Peptide } & \multicolumn{8}{|c|}{ Neutrophil response } \\
\hline & \multicolumn{4}{|c|}{ Cell death } & \multicolumn{4}{|c|}{ Granule release } \\
\hline & \multicolumn{2}{|c|}{1 hour } & \multicolumn{2}{|c|}{6 hours } & \multicolumn{2}{|c|}{1 hour } & \multicolumn{2}{|c|}{6 hours } \\
\hline & Naïve & Activated & Naïve & Activated & Naïve & Activated & Naïve & Activated \\
\hline Linear Bac1 & & & - & - & & & + & + \\
\hline Bac5 & & & - & & & & & + \\
\hline BMAP-34 & + & & $-/+$ & + & & & + & + \\
\hline
\end{tabular}

(+) indicates induction or enhancement; (-) indicates inhibition

Table 4.5 Dose-response summary 2

\begin{tabular}{|c|c|c|c|c|c|c|}
\hline \multirow[b]{3}{*}{ Peptide } & \multicolumn{6}{|c|}{ Neutrophil response } \\
\hline & \multirow{2}{*}{$\begin{array}{c}\text { Migration } \\
\text { Naïve }\end{array}$} & \multicolumn{2}{|c|}{ Respiratory burst } & \multirow{2}{*}{$\begin{array}{c}\text { Phagocytosis } \\
\text { Naïve }\end{array}$} & \multicolumn{2}{|c|}{ Calcium flux } \\
\hline & & Naïve & Activated & & Naïve & Activated \\
\hline Linear Bac1 & + & + & & + & & \\
\hline Bac5 & + & + & - & + & & \\
\hline BMAP-34 & + & - & & + & & \\
\hline
\end{tabular}

(+) indicates induction or enhancement; (-) indicates inhibition 


\subsubsection{LPS-binding activity}

To test the hypothesis of LPS-binding, a standard chromogenic Limulus amoebocyte lysate assay was modified so that serial dilutions of peptide were spiked with a known concentration of LPS. Measurement of free LPS in solution permitted AMP binding parameters to be calculated (Figure 4.11, Table 4.6). All peptides demonstrated LPSbinding activity. Linear Bac1 and BMAP-34 had the greatest LPS-binding capacity with $\mathrm{EC}_{50}$ values of $0.1 \mu \mathrm{M}$. BMAP-28 also had a similarly low $\mathrm{EC}_{50}$ value of $0.2 \mu \mathrm{M}$ contrary to a previous report ( $c f .4 .2 \mu \mathrm{M}$ [161]). Bac1 and Bac5 had $\mathrm{EC}_{50}$ values of $0.4 \mu \mathrm{M}$ and $0.5 \mu \mathrm{M}$, respectively. Indolicidin had the least LPS-binding capacity with an $\mathrm{EC}_{50}$ value of $1.1 \mu \mathrm{M}$. The LPS-binding capacity of LL-37 was also tested to allow these data to be compared with previous reports. LL-37 bound LPS with an $\mathrm{EC}_{50}$ of $0.3 \mu \mathrm{M}$ which was similar to previous reports (cf. $0.36 \mu \mathrm{M}$ [231] and $0.42 \mu \mathrm{M}$ [137]).

\subsubsection{AMP-induced cytokine release from bovine neutrophils}

Neutrophils have a role in cytokine-mediated signalling during the inflammatory response [232]. The strong LPS-binding activity of the bovine peptides led to the hypothesis that the bovine AMPs would augment the release of cytokines from LPSstimulated neutrophils. Culture media from unstimulated or LPS-stimulated neutrophils treated with AMP were tested for the bovine cytokines TNF $\alpha$, IL-1 $\beta$, IL-6, IL-8 and IL-10 by enzyme-linked immunosorbent assay (ELISA). However, control samples without cells, containing only media and AMP gave false positive readings in several of the ELISAs tested. Control samples containing media alone were negative. This strongly indicated that the bovine AMPs were non-specifically binding antibody molecules leading to false-positive readings. This technical problem is discussed further in Appendix 1. Subsequently, investigation into cytokine release by neutrophils was not further pursued. 

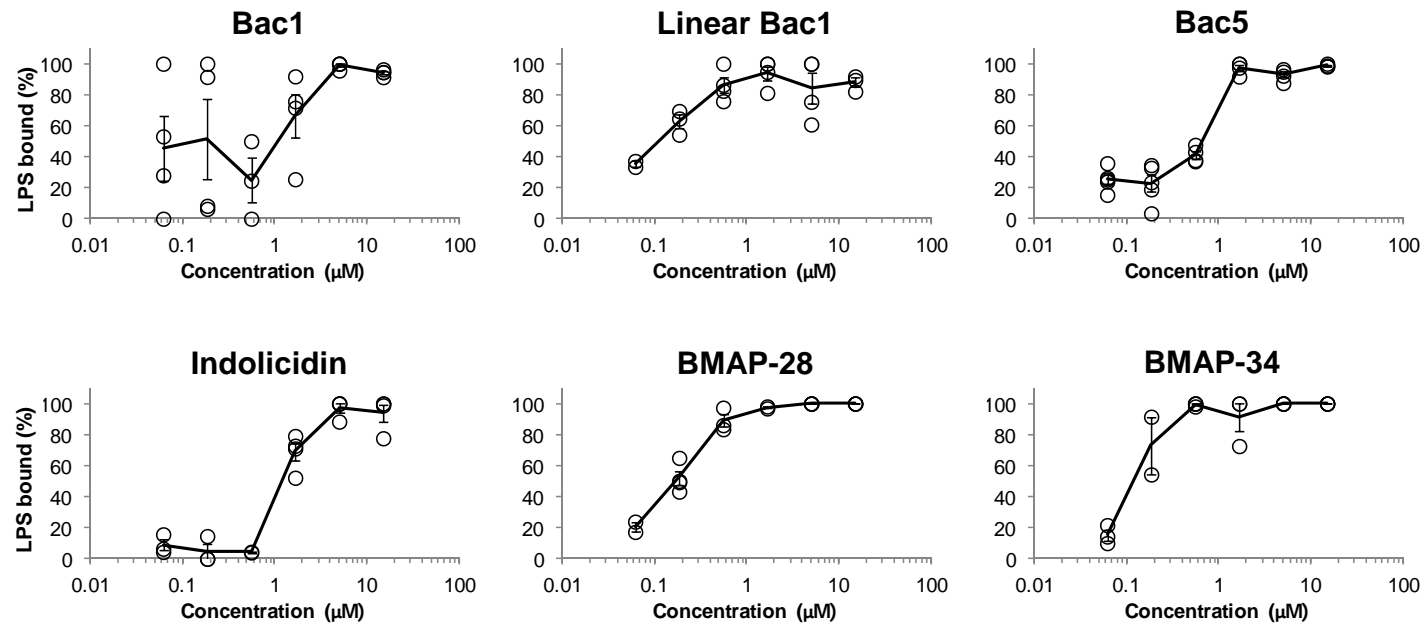

Figure 4.11 LPS-binding activity of bovine cathelicidin AMPs

Serial dilutions of peptide were spiked with $0.5 \mathrm{EU}$ and incubated for 30 minutes at $37^{\circ} \mathrm{C}$. Free LPS was measured with a chromogenic Limulus amoebocyte lysate assay kit. Data represent the mean \pm SEM for at least two independent experiments performed in duplicate.

Table 4.6 LPS-binding statistics

\begin{tabular}{|c|c|c|c|}
\hline & Best-fit values & $95 \%$ Confidence Intervals & Goodness of Fit \\
\hline Peptide & $\mathrm{EC}_{50}(\mu \mathrm{M})$ & $\mathrm{EC}_{50}(\mu \mathrm{M})$ & $\mathbf{R}^{2}$ \\
\hline Bac1 & 0.4 & 0.15 to 1.08 & 0.16 \\
\hline Linear Bac1 & 0.1 & 0.07 to 0.17 & 0.61 \\
\hline Bac5 & 0.5 & 0.35 to 0.68 & 0.89 \\
\hline Indolicidin & 1.1 & 0.68 to 1.70 & 0.87 \\
\hline BMAP-28 & 0.2 & 0.12 to 0.19 & 0.93 \\
\hline BMAP-34 & 0.1 & 0.07 to 0.18 & 0.80 \\
\hline LL37 & 0.3 & 0.19 to 0.49 & 0.81 \\
\hline
\end{tabular}

Values and statistics were calculated from non-linear regression analysis carried out using Prism v5.0 (GraphPad Software, Inc.). 


\subsection{Discussion}

The focus of this chapter was to determine the effects of selected bovine cathelicidin AMPs on neutrophil function and LPS-binding capacity, testing the hypothesis that cathelicidin peptides have differential activities, dependent on AMP structure and neutrophil activation status.

\subsubsection{The effects of bovine cathelicidin AMPs on neutrophil function}

The six bovine cathelicidin AMPs selected for the primary screen of neutrophil specific activity include peptides with different sequence and structure properties (Table 4.1). The results of these studies show that individual bovine cathelicidin peptides have the ability to exert different effects on naïve and activated neutrophils in a sequence and structure dependent manner (Table 4.2, Table 4.3, Table 4.4 and Table 4.5).

The $\beta$-turn form of Bac1, indolicidin and BMAP-28 were eliminated from further analysis in dose-response assays. In the primary screen analysis these peptides exhibited minimal ability to modify neutrophil function or induced cell death. Indolicidin and the $\beta$-turn form of Bac1 are cytotoxic to rat and human T lymphocytes [159] but were not cytotoxic to bovine neutrophils in this study. The only significant activity identified with the $\beta$-turn form of Bac1 was an ability to enhance the migration rate of naïve neutrophils. An increase in migration rate stimulated by Bac1 would serve to enhance directed neutrophil infiltration to sites of infection, in a similar manner to chemoattractants such as leukotriene B4, C5a and IL-8 [233]. At inflammatory foci, the combined antimicrobial effects and enhancement of neutrophil migration by Bac1 would also indirectly aid phagocytic clearance of bacteria.

Indolicidin is structurally unique in that it contains an unusually high content of tryptophan [121]. This unique structure is very divergent from the other bovine AMPs and may explain the ability of indolicidin to trigger neutrophil respiratory burst without affecting other AMP sensitive neutrophil activities. A possible mechanism for this selective activity is unclear although indolicidin has been shown to cross biological membranes [234], and may therefore exert its effects intracellularly. 
Of all the AMPs tested, the $\alpha$-helical peptide BMAP-28 exhibited the most striking 'activity' profile, and induced significant changes in all of the pro-inflammatory neutrophil functions tested in the primary screen assays. However, BMAP-28 is highly cytotoxic, a property which has also been observed in cell lines from non-bovine species $[158,160]$, and induced significant cell death in both naïve and activated bovine neutrophils. This suggests the BMAP-28-induced modulation of neutrophil function is primarily the result of cell death. Interestingly, bovine RBCs are tolerant to BMAP-28 toxicity [127] which suggests BMAP-28-induced neutrophil death may be a specific function to promote clearance of spent neutrophils from inflammatory foci.

The in-depth analysis of linear Bac1, Bac5 and BMAP-34 produced interesting results (Figure 4.12). With slight modifications to several of the assays to better mimic conditions found in vivo, these peptides exhibited dose-dependent effects on neutrophil function. Both linear and cyclic structural variants of the Bac1 peptide were able to enhance migration indicating amino acid composition, rather than structure, is a defining factor for Bac1 enhanced migration. However, the dose-response analysis showed the linear form was able to enhance migration at much lower concentrations than the $\beta$-turn form, suggesting some structural aspect of the linear configuration, as well as composition, results in a more potent chemokinetic effect. Linear Bac1 also produced a dose-dependent increase in neutrophil respiratory burst, which conversely did not occur in the presence of the $\beta$-turn form. Similar to indolicidin, liposome interaction studies indicate the linear form of Bac1 is effective at penetrating lipid bilayers [235]. Therefore linear Bac1 may activate ROS production in a similar manner to indolicidin via a cytosolic mechanism. The enhancement of phagocytosis observed in the dose-response analysis may likewise occur via an intracellular mechanism.

Bac5 was able to dose-dependently enhance migration, phagocytosis and ROS production in naïve neutrophils. In activated neutrophils Bac5 inhibited ROS production, enhanced cell survival and promoted secondary granule release. Taken together, these results indicate a pro-inflammatory effect which regulates the production of ROS to ensure neutrophils remain viable, active and capable of functioning appropriately during an immune response. Migration, degranulation and respiratory burst induced via intracellular calcium mobilisation is a hallmark of receptor mediated 
activation [236,237]. The absence of detectable calcium flux in Bac5-stimulated neutrophils indicates a receptor-independent mode of action. The opposing inhibitory effect of Bac5 on PMA-induced ROS production, which occurs via intracellular signalling, also supports a receptor-independent mode of action. The inhibition of PMAinduced ROS production is similar to that reported for the structurally related prolinerich porcine cathelicidin PR-39 [154,155], suggesting a common mechanism of inhibition by proline-rich peptides. Further studies of this potential mode of action are covered in Chapter 5.

The $\alpha$-helical peptides BMAP-34 and BMAP-28 have similar structure, however BMAP-34 is amphipathic, containing positively and negatively charged amino acids and hydrophobic amino acids along opposite sides of the $\alpha$-helical axis [196]. In contrast BMAP-28 has an unstructured hydrophobic C-terminal region and lacks negative amino acids [127]. Interestingly, in the primary screen assays BMAP-34 induced membrane permeabilisation and cytotoxicity only in activated neutrophils. The difference in structure and the extensive reorganisation of membrane components during cellular activation [238] may contribute to the differential effects observed for these $\alpha$-helical peptides. This suggests evolution of the BMAP-34 gene has led to a peptide sequence which specifically 'targets' activated or spent neutrophils, inducing cell death and promoting clearance in a more refined manner compared to BMAP-28.

Like linear Bac1 and Bac5, BMAP-34 also enhanced migration and phagocytosis. However, a mild inhibitory effect on naïve cell ROS production was also observed at low concentrations. As ROS production can be a mediator of programmed cell death [239], inhibition of ROS at low BMAP-34 concentrations correlates with enhanced survival of naïve cells after 6 hours exposure to the peptide. At low concentrations BMAP-34 also promoted large granule release. Large granules in bovine neutrophils contain the pro-forms of cathelicidin [45], therefore this response may represent a mechanism of self-regulating cathelicidin release. Together, these results indicate BMAP-34 may have a regulatory role to maintain naïve cells and promote inflammatory functions at low concentrations while aiding removal of dead cells and debris at high concentrations. 
Previous studies with other AMPs link their biological activities with cell permeabilisation, an event that triggers non-specific calcium influx [152,160]. LL-37 and the bovine peptides BMAP-27 and BMAP-28 have all been shown to elevate intracellular calcium levels in conjunction with ROS production and cell death in proliferating human lymphocytes [152,160]. Interestingly, linear Bac1, Bac5 and BMAP-34 were unable to induce changes in neutrophil intracellular calcium levels indicating that these peptides orchestrate their effects via calcium-independent signalling pathways. It also indicates that these AMPs are unlikely to be causing nonspecific membrane permeabilisation.

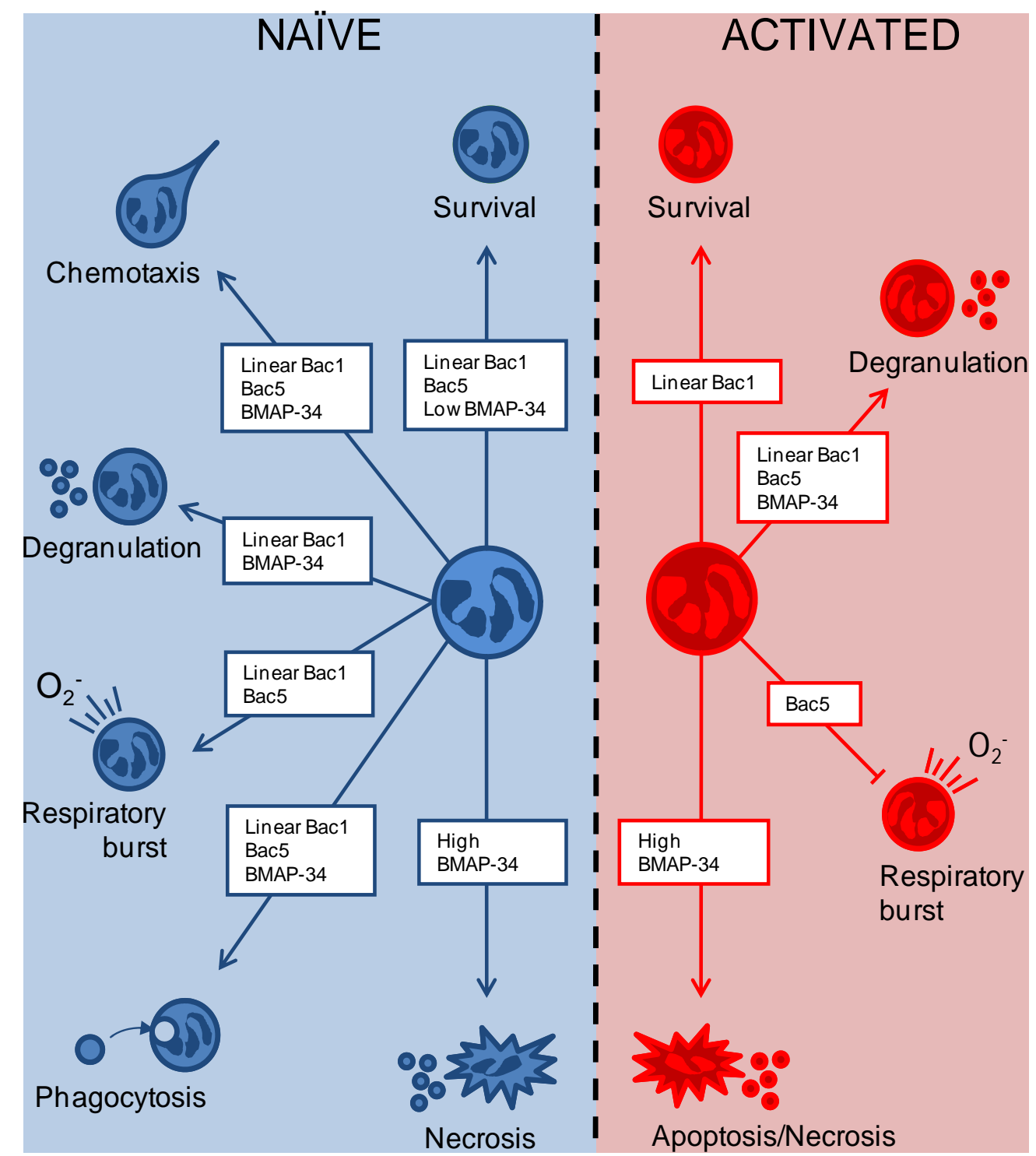

Figure 4.12 Summary of the effects of linear Bac1, Bac5 and BMAP-34 on neutrophil function in vitro 


\subsubsection{LPS-binding activity}

The ability to bind LPS is a common feature of cathelicidin AMPs (Table 4.7). Electrostatic interactions occur between the cationic AMPs and negatively charged LPS molecules. Therefore the presence of positive amino acids, and the absence of negative amino acids are the main determinants of the AMP binding affinity [240]. Also, the relevance of structure is demonstrated by the different binding affinities of the linear and $\beta$-turn forms of Bac1, and the generally higher but differential binding affinity of the $\alpha$-helical peptides. AMPs with low LPS-binding $\mathrm{EC}_{50}$ values correlate with an ability to alter host cell responses to LPS in vitro, or protection against endotoxic shock in vivo $[169,177,241,242]$. This has been demonstrated for many cathelicidin AMPs (see Chapter 1, Table 1.3 and Table 1.4), indicating the bovine cathelicidin AMPs used in these studies may have comparable activity. Measurement of cytokine release by LPS-stimulated neutrophils in the presence of AMPs may have supported this hypothesis. Unfortunately cytokine release could not be measured accurately. This was due to non-specific binding of the peptides to either capture or detection antibodies of several different bovine-specific ELISAs. The non-specific binding was robust enough to produce consistently significant false positive readings making measurement of actual cytokines impossible. Measurement of cytokine mRNA changes may have been a viable alternative, although not a direct measure of release per se. The non-specific peptide-antibody interaction is discussed further in Appendix 1.

Table 4.7 Examples of cathelicidin AMPs that bind LPS

\begin{tabular}{|lccccc|}
\hline Peptide & Species & Structure & Method & EC $_{50}(\boldsymbol{\mu M})$ & Reference \\
\hline Bac5 & Caprine (goat) & Linear & Quantitative LAL assay & 4.0 & {$[122]$} \\
Bac7 & Bovine (cow) & Linear & Quantitative LAL assay & $\sim 1.5$ & {$[241]$} \\
Indolicidin & Bovine (cow) & Linear & Polymyxin B displacement assay & 8.5 & {$[243]$} \\
Indolicidin & Bovine (cow) & Linear & Polymyxin B displacement assay & 18 & {$[244]$} \\
BMAP-28 & Bovine (cow) & a-helical & Quantitative LAL assay & 4.2 & {$[161]$} \\
LL-37 & Human & a-helical & Quantitative LAL assay & 0.36 & {$[231]$} \\
LL-37 & Human & a-helical & Quantitative LAL assay & 0.42 & {$[137]$} \\
CAP-11 & Guinea Pig & a-helical & FITC-LPS binding to RAW 264.7 cells & 0.053 & {$[245]$} \\
RL-37 & Rhesus monkey & a-helical & Quantitative LAL assay & 4.44 & {$[137]$} \\
SMAP-29 & Ovine (sheep) & $\alpha$-helical & Quantitative LAL assay & 2.56 & {$[138]$} \\
SMAP-29 & Ovine (sheep) & $\alpha$-helical & Quantitative LAL assay & 3.2 & {$[242]$} \\
\hline
\end{tabular}




\subsection{Conclusions}

The results of this chapter demonstrate that bovine cathelicidin AMPs have differential effects on neutrophil innate immune function and that these activities are dependent on neutrophil activation state and peptide structure. This suggests the peptides have a range of different immunomodulatory roles during infection and inflammation. Furthermore, all the peptides have the ability to bind LPS. This ability of each peptide to bind LPS with relatively high affinity may alter recognition of PAMPs by immune cells, although it is unclear whether this function would be facilitative or preventative. Measurement of cytokine release by LPS-stimulated neutrophils in the presence of AMPs may have clarified this observation but unfortunately cytokine release could not be measured accurately.

Interestingly, the selected AMPs were able to modify a number of neutrophil functions. While the effects of BMAP-28 were attributable to toxicity, other functions were altered by AMPs in the absence of toxic effects. Migration and ROS production were enhanced by several peptides while ROS production was inhibited by others. When investigated in further detail, linear Bac1, Bac5 and BMAP-34 were able to dose-dependently induce or inhibit several key neutrophil functions including migration, degranulation, respiratory burst and phagocytosis, indicating significant roles in differential modulation of immune responses. The results of this chapter also suggest some bovine cathelicidins are able to cross neutrophil membranes without significant disruption to cellular homeostasis, possibly eliciting their effects intracellularly. Due to the ability of Bac5 to differentially modify neutrophil respiratory burst, the potential of Bac5 to act via an intracellular mechanism to alter ROS production was investigated in Chapter 5. 
Chapter 5: The Mechanism of Bac5 Regulation of the Respiratory Burst 


\subsection{Introduction}

Cathelicidin AMPs are able to penetrate eukaryotic membranes. In most cases these peptides have amphipathic $\alpha$-helical structures and cause a membranolytic effect which induces cell death [157-160,173]. However, amphipathicity and flexibility of peptides are determinants of their ability to translocate across membranes [246]. Linear peptides rich in proline and arginine have been shown to be internalised by eukaryotic cells, either by passive diffusion or by endocytosis, without causing lysis [247,248]. Examples of these peptides include bovine Bac5 and Bac7, and porcine PR-39.

Bac5 has been shown to efficiently translocate into murine fibroblasts [247], and Bac7 into murine fibroblasts and human lymphoma cells [247,248] where they promote cellular proliferation. Porcine PR-39 has been studied in more detail. The intracellular effects of this peptide include modulation of gene expression [183] and promotion of angiogenesis by inhibiting degradation of hypoxia-inducible factor 1- $\alpha$ [184]. PR-39 also inhibits apoptosis by down-regulating caspase-3 and inducing inhibitor of apoptosis-2 [185,186], and inhibits ROS production by binding to $\mathrm{p} 47^{\text {phox }}[154,155]$.

The proline-rich nature of bovine Bac5 and Bac7, and porcine PR-39 allow them to mediate protein-protein interactions that rely on consecutive proline repeat motifs for signalling [249,250]. For example, PR-39 binds to the Src homology domain of the p4 $7^{\text {phox }}$ subunit, a domain that recognises proline-rich ligand motifs. In Chapter 4 , Bac5 was shown to inhibit PMA-induced ROS production. PMA acts via an intracellular mechanism to induce ROS production. Bac5 is known to translocate into eukaryotic cell lines, and shares structural similarities with PR-39, which directly interacts with components of the NADPH oxidase complex. This led to the hypothesis that: Bac5 acts via an intracellular mechanism to regulate neutrophil respiratory burst. 


\subsection{Aims}

- Determine whether Bac5 crosses the neutrophil membrane and accumulates intracellularly.

- Assess the effect of Bac5 on intracellular ROS production in neutrophils.

- Identify intracellular Bac5-protein interactions that might contribute to the mechanism of Bac5-mediated regulation of neutrophil respiratory burst. 


\subsection{Results}

\subsubsection{Assessment of Bac5 internalisation}

A synthetic Bac5 peptide conjugated to FITC was used to investigate Bac5 internalisation. Naïve neutrophils were exposed to Bac5-FITC for up to 60 minutes then analysed by confocal microscopy. To obtain semi-quantitative data on the rate of internalisation of the peptide, neutrophil uptake was assessed at different times by flow cytometry. Confocal microscopy showed Bac5-FITC was internalised by naïve neutrophils (Figure 5.1). The extent of internalisation varied, with some neutrophils internalising more peptide than others. The peptide was localised to the cytoplasm of most cells in a diffuse, non-specific pattern and did not appear to enter the nuclei. In a minority of cells Bac5-FITC appeared to be localised to vesicles, suggesting an active uptake mechanism of peptide internalisation.

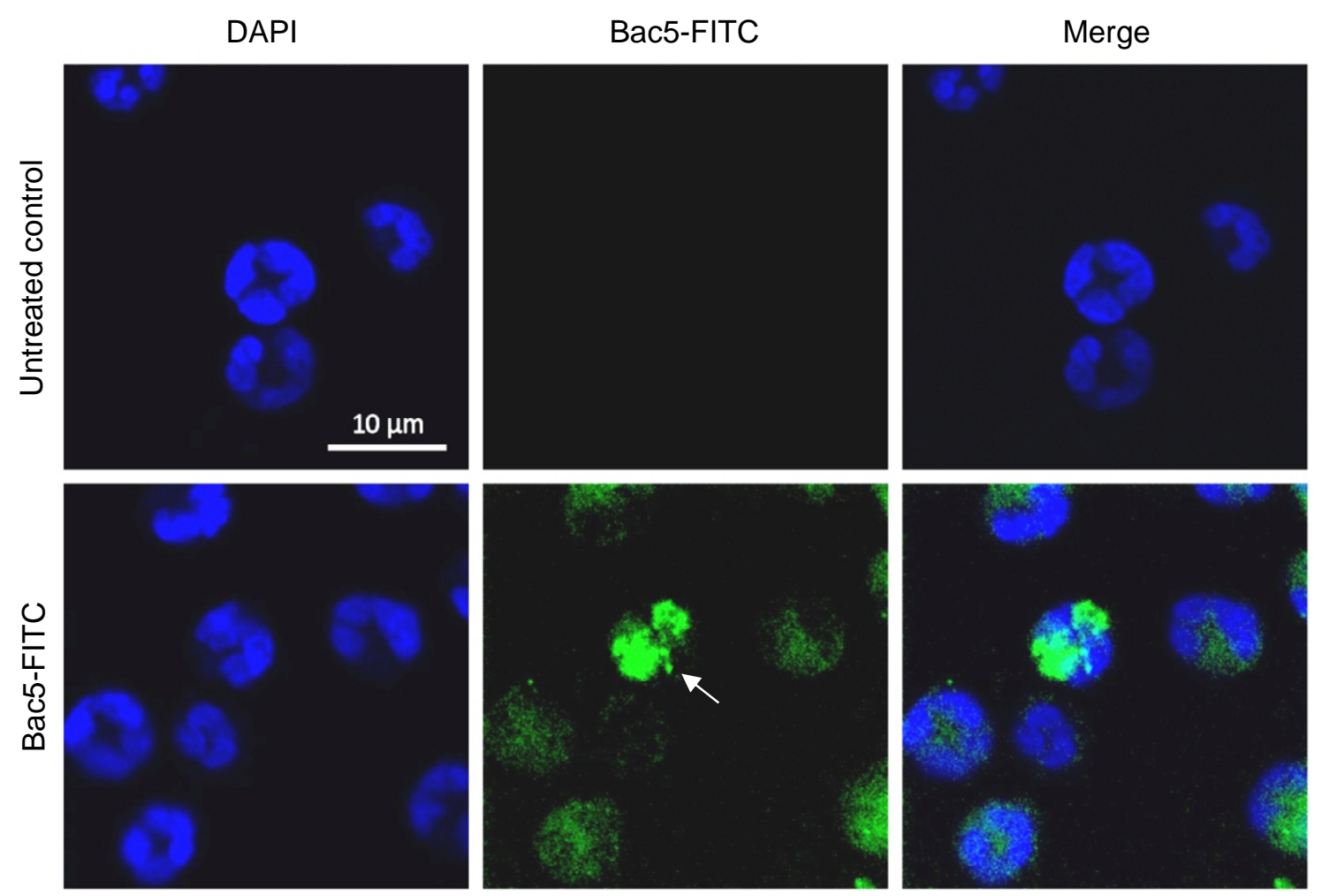

Figure 5.1 Confocal microscopy of Bac5-FITC-treated neutrophils

Bovine neutrophils were seeded onto coverslips and treated with $2.5 \mu \mathrm{M}$ Bac5-FITC for 60 minutes. Bac5-FITC signal was confined to the cytoplasm of most cells. In some cells Bac5-FITC appeared to be confined to vesicles (arrow). Images are representative of two independent experiments. 
Flow cytometry analysis showed peptide internalisation occurred in a time and dosedependent manner (Figure 5.2). At $5 \mu \mathrm{M}$ or greater, significant amounts of peptide were present after five minutes. At $2.5 \mu \mathrm{M}$ or greater, significant internalisation occurred after 30 minutes. After five minutes, there appeared to be a linear relationship of internalisation with respect to time, indicating a constant rate of internalisation of Bac5FITC, either by passive diffusion, active uptake, or both processes simultaneously (Figure 5.2 B).

A
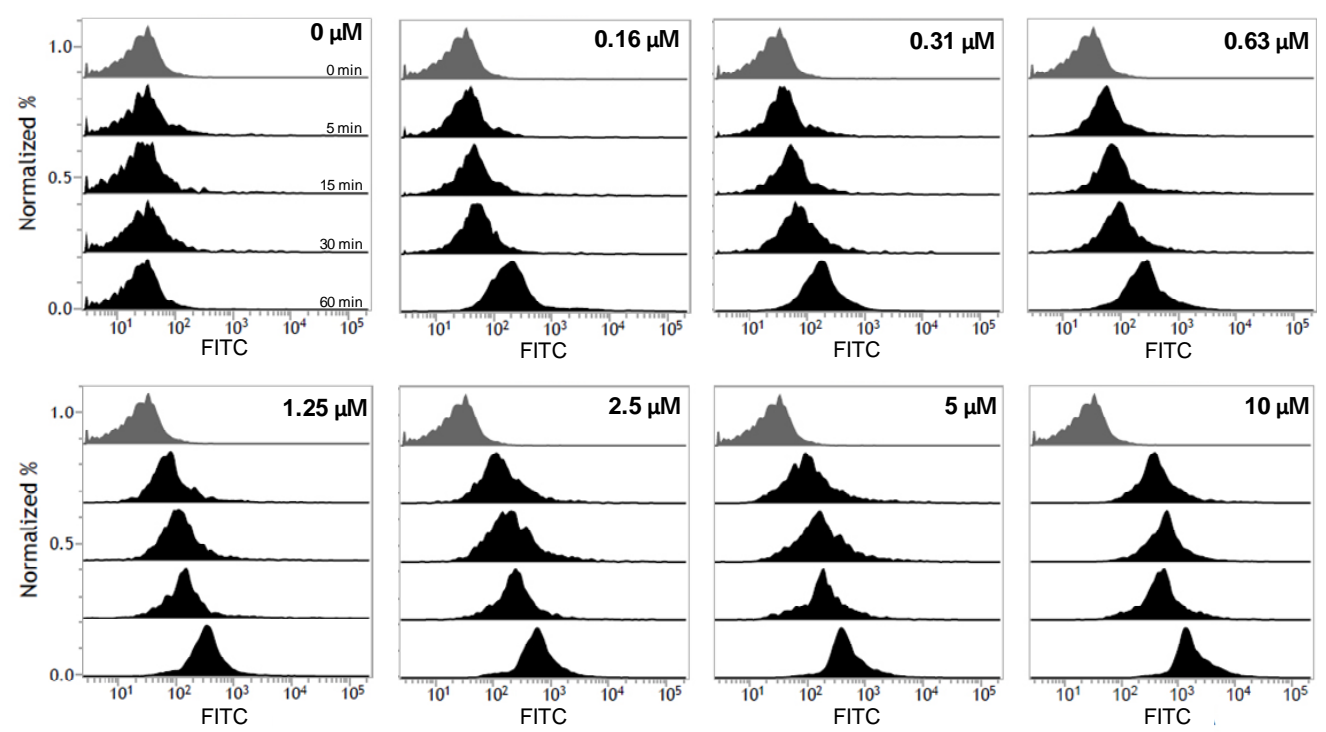

B

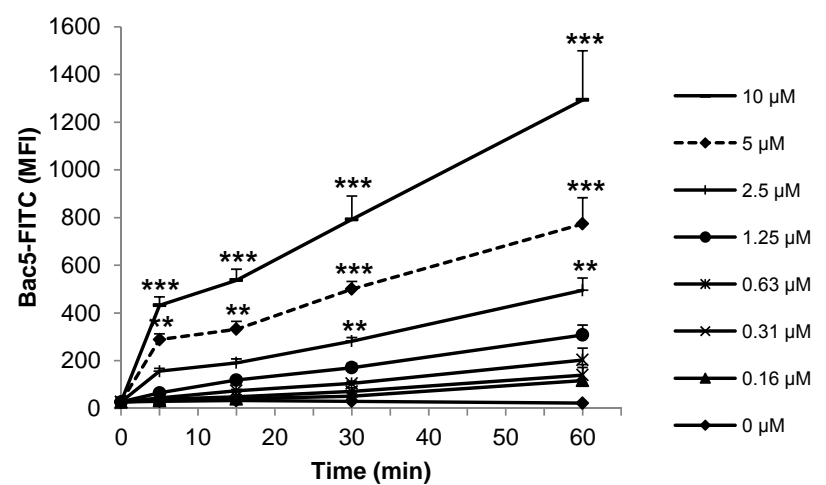

\section{Figure 5.2 Flow cytometry analysis of Bac5-FITC-treated neutrophils}

Neutrophils were exposed to increasing concentrations of Bac5-FITC for 5, 15, 30 and 60 minutes. Extracellular FITC fluorescence was quenched with trypan blue. Cells were washed and fixed before flow cytometry analysis. (A) Representative histogram analysis. (B) Dose-time-response analysis. Data represent the mean \pm SEM for three independent experiments performed in duplicate. Statistical significance was determined by one-way ANOVA with Tukey's post-test comparing treatments with control. ** $P<0.01, * * * P<0.001$. 


\subsubsection{Bac5 induces intracellular ROS production}

Because Bac5 was able to induce extracellular ROS production (Chapter 4, section 4.3.3.4) and was readily found in the cytoplasm of neutrophils, intracellular ROS production was also assessed. Naïve neutrophils were treated with the intracellular fluorescent ROS indicator CellROX Deep Red and Bac5-FITC. The cells were analysed by flow cytometry to determine whether Bac5 induced intracellular ROS production. For flow cytometry analysis, neutrophils were gated by forward and side scatter properties (Figure 5.3 A, P1) and Bac5-FITC-positive cells gated (P2) according to FITC-negative cells in untreated controls. Histograms of Bac5-FITC-positive cells displaying the median fluorescence intensity (MFI) of CellROX Deep Red fluorescence were used for dose-response analysis (Figure $5.3 \mathrm{~B}$ ). The analysis of Bac5-FITC positive cells showed naïve neutrophils treated with Bac5 produced a dose and timedependent increase in intracellular ROS production (Figure 5.3 C). The increase was significant after 30 minutes with cells treated with at least $5 \mu \mathrm{M}$ Bac5-FITC, and after 60 minutes with at least $2.5 \mu \mathrm{M}$. The flow cytometry analysis also affirmed that cells positive for intracellular ROS production were also positive for Bac5-FITC, indicating that internalisation of Bac5 was associated with intracellular ROS production. 
A
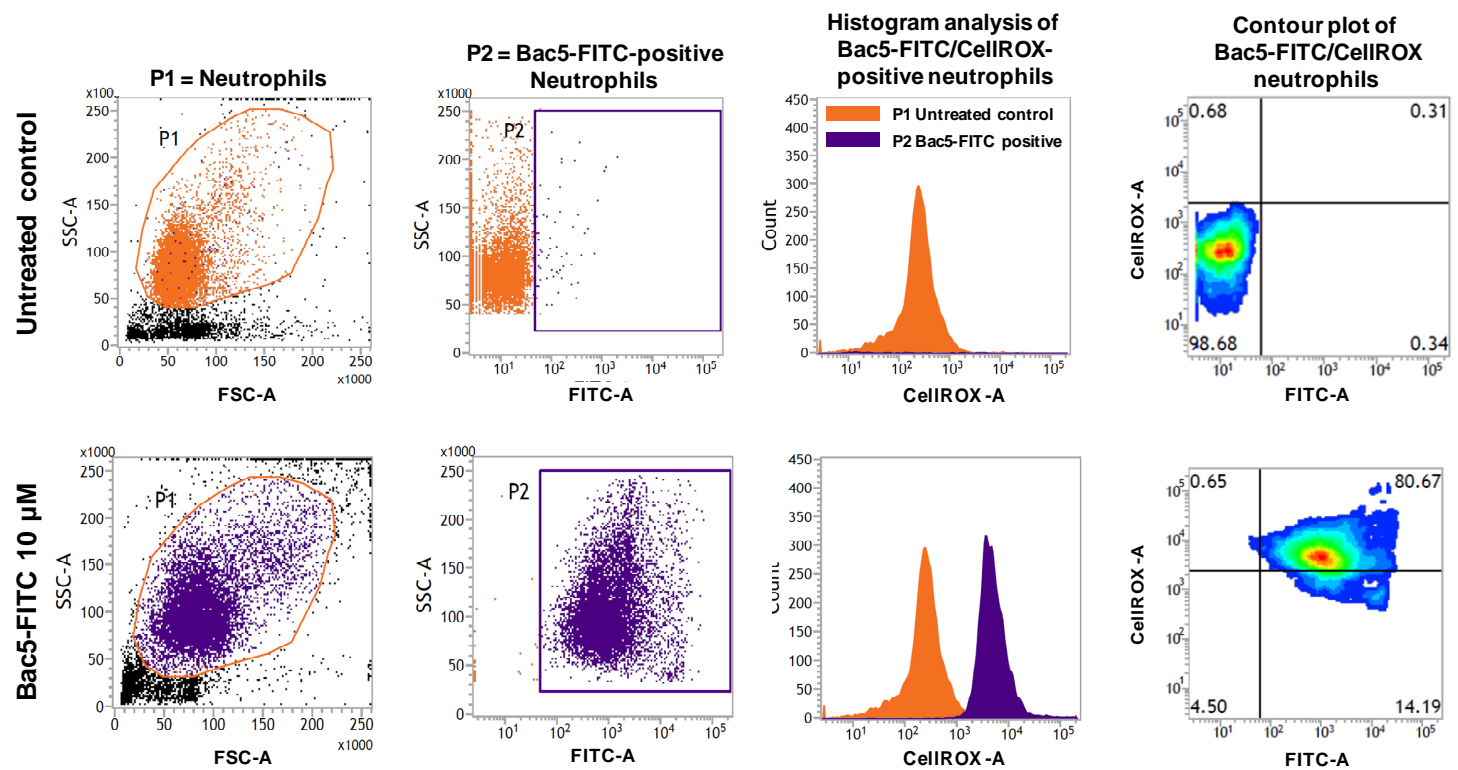

B

\section{Intracellular ROS production in naïve neutrophils}
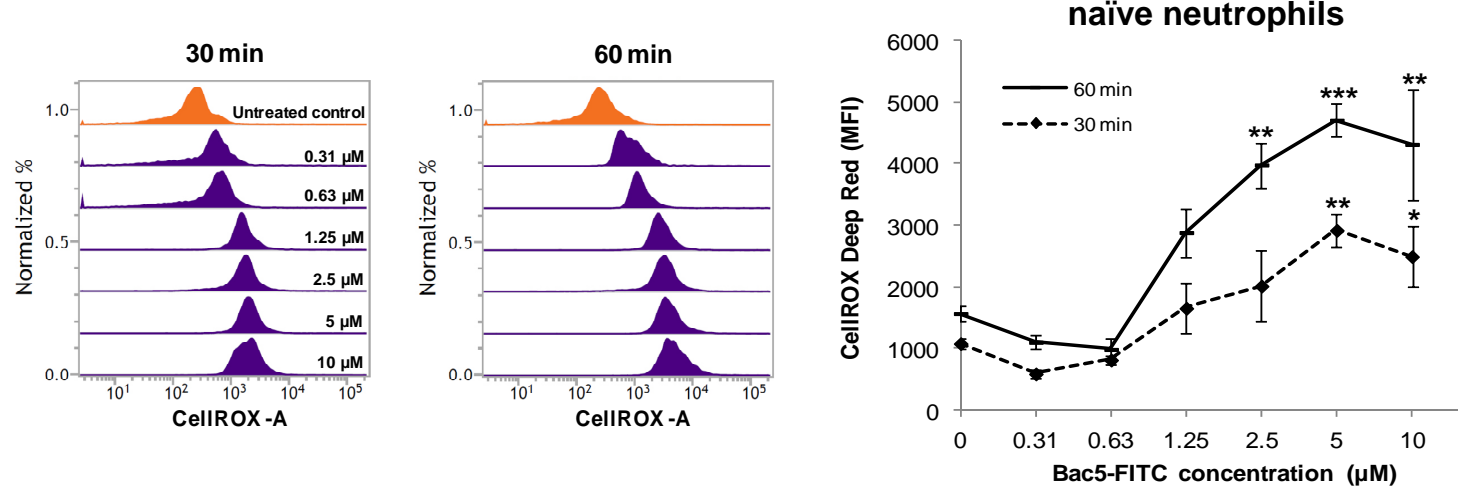

Figure 5.3 Flow cytometry analysis of Bac5-induced intracellular ROS production

The fluorescent intracellular ROS indicator CellROX Deep Red was used to measure intracellular ROS induced by Bac5-FITC using flow cytometry. (A) Dotplots, histograms and contour plots representing the gating strategy for Bac5-FITC-positive/CellROX-positive neutrophils after 60 minutes. (B) Stacked histograms of Bac5-FITC-positive/CellROX-positive neutrophils at 30 and 60 minutes. (C) Doseresponse curves for intracellular ROS production induced by Bac5-FITC. Data represent the mean \pm SEM for three independent experiments performed in duplicate. Statistical significance was determined by oneway ANOVA with Tukey's post-test comparing treatments with control. * $P<0.05$, ** $P<0.01$, *** $P<0.001$. 


\subsubsection{Bac5 colocalises with intracellular ROS}

Confocal microscopy of Bac5-FITC and CellROX Deep Red-treated cells showed that intracellular ROS production was diffuse throughout the cytoplasm but also appeared to be localised in vesicles (Figure 5.4). The CellROX Deep Red signal was constantly localised with a strong Bac5-FITC signal in either cytoplasmic or vesicular locations. Untreated cells showed minor background CellROX Deep Red fluorescence. Together these observations supported the data from the flow cytometry analysis.

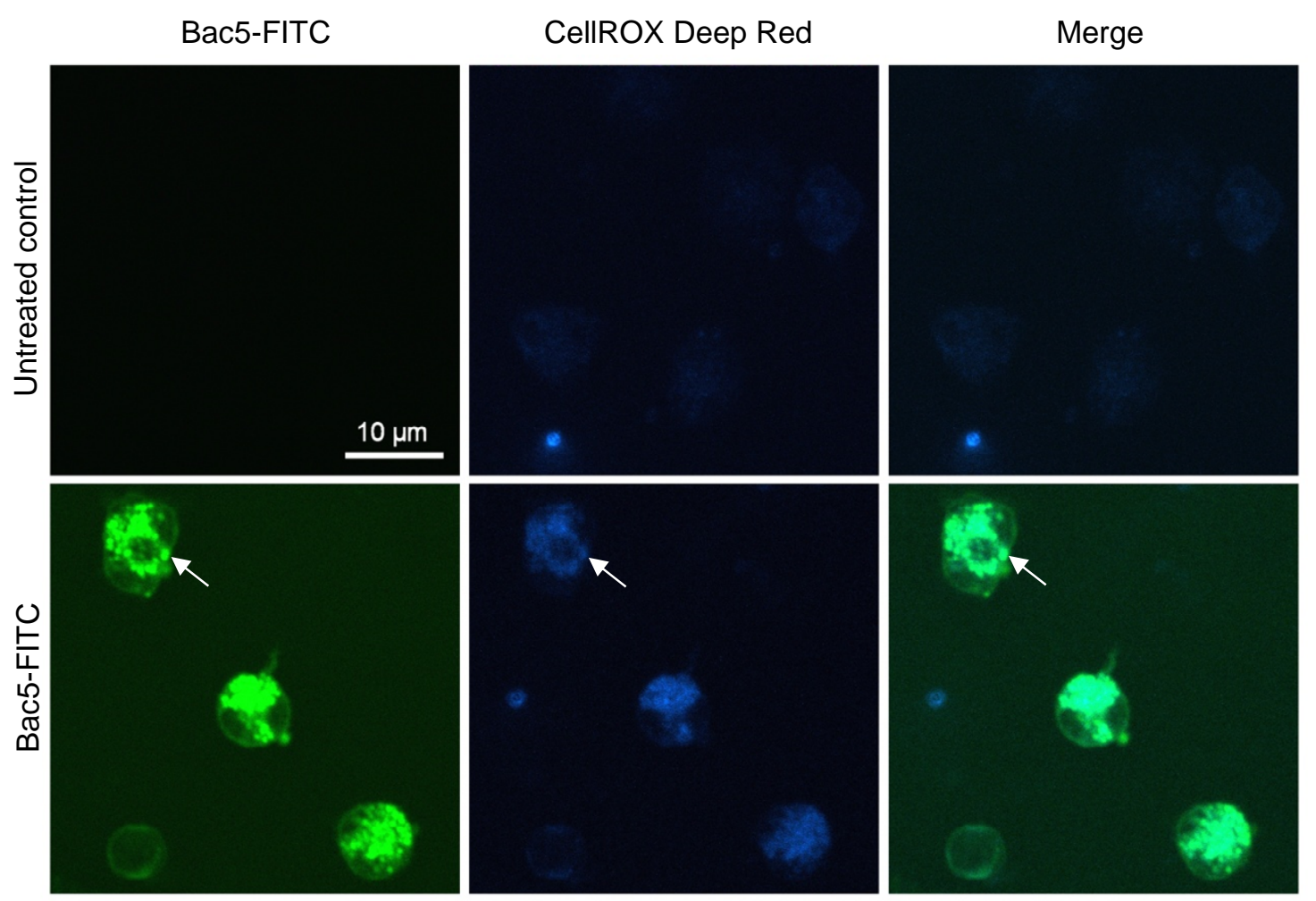

Figure 5.4 Confocal microscopy of Bac5 and intracellular ROS colocalisation

Neutrophils were seeded onto glass coverslips and incubated with $2.5 \mu \mathrm{M}$ Bac5-FITC (green) for 60 minutes in the presence of CellROX Deep Red (blue). White arrows indicate vesicular colocalisation (cyan) of Bac5-FITC and ROS production. Images are representative of two independent experiments. 


\subsubsection{Intracellular targets of Bac5}

The internalisation of Bac5-FITC suggested that Bac5 was acting intracellularly to modulate neutrophil function. Extracellular and intracellular ROS production induced by Bac5 also suggested Bac5 was interacting with the NADPH oxidase complex or proteins associated with oxidase activation. For active ROS production, cytosolic subunits of the oxidase assembly must translocate to the membrane bound subunits which are subsequently phosphorylated by kinases to form an active NADPH oxidase complex. The cytosolic subunit $\mathrm{p} 47^{\mathrm{phox}}$ is essential for oxidase activity in response to agonists $[251,252]$. To investigate the role of Bac5 in ROS production, measurement of the translocation of $\mathrm{p} 47^{\mathrm{phox}}$ was used to assess whether Bac5 upregulates membrane expression of active NADPH oxidase. Far-western blotting of neutrophil lysate and MS analysis was then used to assess which intracellular proteins Bac5 associates with.

\subsubsection{Bac5-induced ROS production does not involve $\mathrm{p} 47^{\text {phox }}$ translocation}

Bac5 was able to consistently induce ROS production at $2.5 \mu \mathrm{M}$, therefore this concentration was used to stimulate naïve neutrophils to produce ROS for 30 or 60 minutes. The cells were then lysed and separated into membrane and cytosol protein fractions and $\mathrm{p} 47^{\mathrm{phox}}$ content assessed by western blot. After adjusting for protein loading (Figure $5.5 \mathrm{~A}$ ) and $\mathrm{p} 47^{\text {phox }}$ signal (Figure $5.5 \mathrm{~B}$ ), localisation of $\mathrm{p} 47^{\text {phox }}$ was compared (Figure $5.5 \mathrm{C}$ ). A significant change in the translocation of cytosolic $\mathrm{p} 47^{\text {phox }}$ to the membrane was not observed at either 30 or 60 minutes compared to untreated neutrophils. Oddly, a trend toward increased cytosolic $\mathrm{p} 47^{\text {phox }}$ was observed 30 minutes after Bac5 treatment, which dissipated after 60 minutes. How this translates into increased NADPH oxidase activity is unclear, although despite contrasting with the expected outcome, there may be the potential for Bac5 to modulate NADPH oxidase assembly or disassembly. 
A

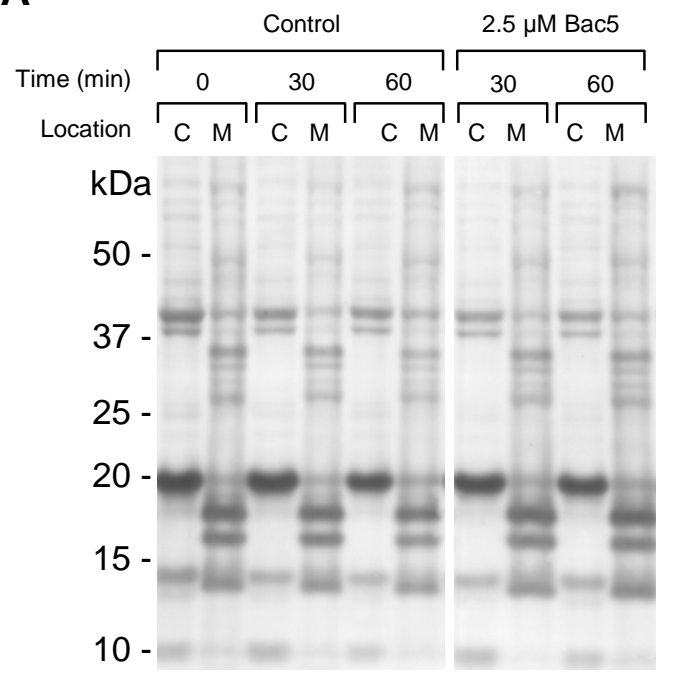

B
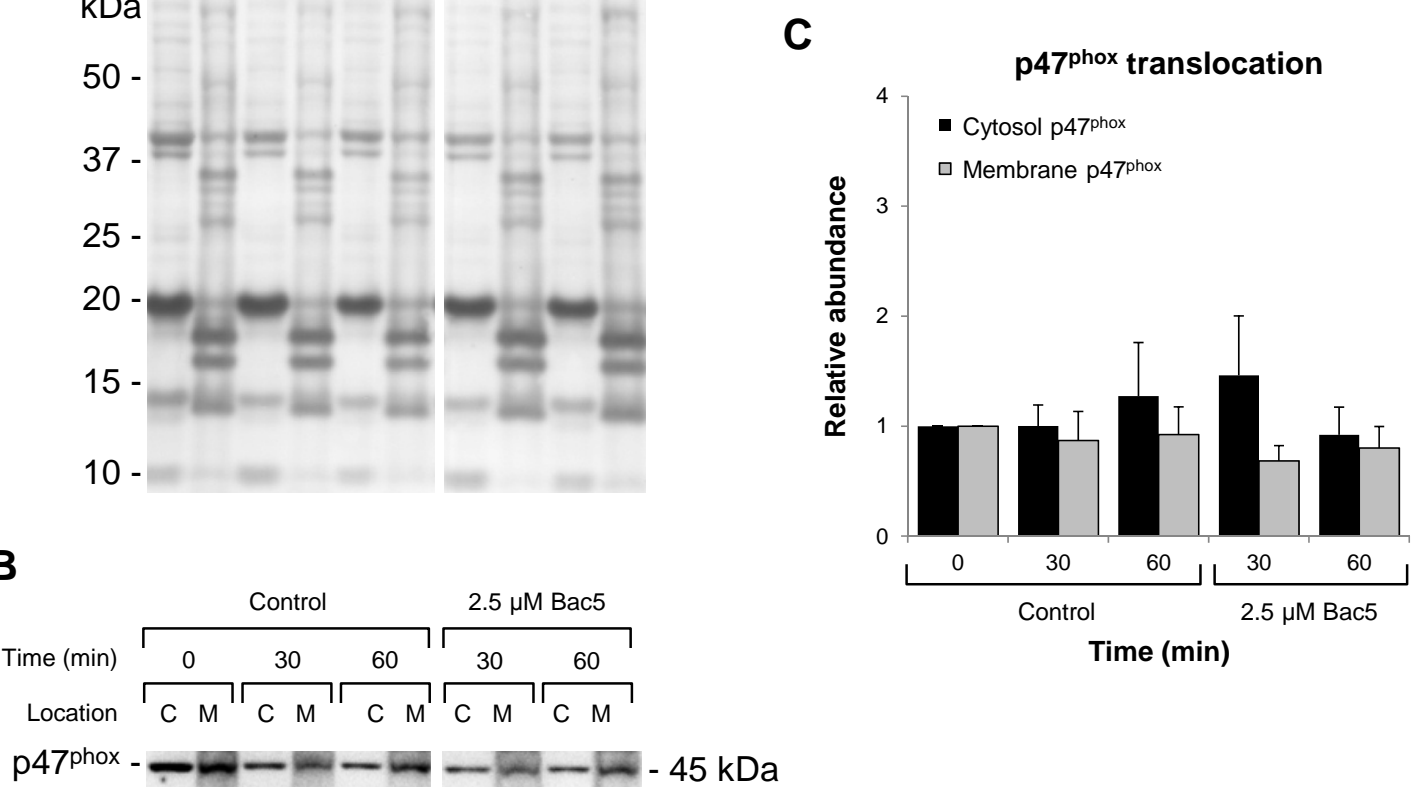

\section{Figure 5.5 Western blot analysis of $\mathrm{p}^{7^{\text {phox }}}$ translocation}

Naïve neutrophils were treated with $2.5 \mu \mathrm{M}$ Bac5 for 30 or 60 minutes. The cells were lysed, separated into cytosol and membrane fractions and $30 \mu \mathrm{g}$ protein probed by western blot using anti-p $47^{\text {phox }}$ antibody. (A) Representative Ponceau S stain for loading controls. (B) Representative anti-p47 $7^{\text {phox }}$ antibody probe. (C) Semi-quantitative analysis of the relative abundance of $\mathrm{p} 47^{\text {phox }}$ in cytosol and membrane fractions. Data represent the mean \pm SEM for three independent experiments. No statistical significance was found using a one-way ANOVA and Tukey's post-test. C, cytosol; M, membrane. 


\subsubsection{Bac5 binds to several intracellular neutrophil proteins}

To investigate potential intracellular Bac5-protein interactions, untreated naïve neutrophils were lysed in the presence of protease inhibitors and the insoluble material removed. Cleared lysate was separated by SDS-PAGE and Bac5-FITC used as the probe in far-western blot analysis. Two membranes were analysed in parallel. Ponceau S stain indicated equivalent loading of neutrophil lysate for each membrane (Figure 5.6 A). After 2 hours incubation, Bac5-FITC had bound with a strong signal corresponding to a protein at approximately $43 \mathrm{kDa}$ (Figure $5.6 \mathrm{~B}$, Blot 1). Minor signals from bands at around $21 \mathrm{kDa}$ were also present. To determine if the interaction was Bac5-specific, a duplicate blot was analysed in parallel and initially blocked with unlabelled Bac5 prior to probing with Bac5-FITC. This produced a reduction in signal from the $43 \mathrm{kDa}$ band as well as a visible reduction in signal from a band at $22 \mathrm{kDa}$ (Figure 5.6 B, Blot 2). The remaining bands at approximately $21 \mathrm{kDa}$ did not appear to have a reduced Bac5-FITC signal and therefore were not analysed by LC-MS/MS.

\subsubsection{3 $\beta$-actin is an intracellular target of Bac5}

To identify the proteins to which Bac5-FITC bound to in the far-western blot analysis, a replicate of neutrophil lysate was electrophoresed on the same polyacrylamide gel and stained with CBB G-250 (Figure 5.6 C). The corresponding bands at $43 \mathrm{kDa}$ (MS1) and $22 \mathrm{kDa}(\mathrm{MS} 2)$ were removed and prepared for analysis by LC-MS/MS. A peptide mass fingerprint mass spectrum was generated and database searches against this mass spectrum showed several hits to neutrophil proteins (Table 5.1). 

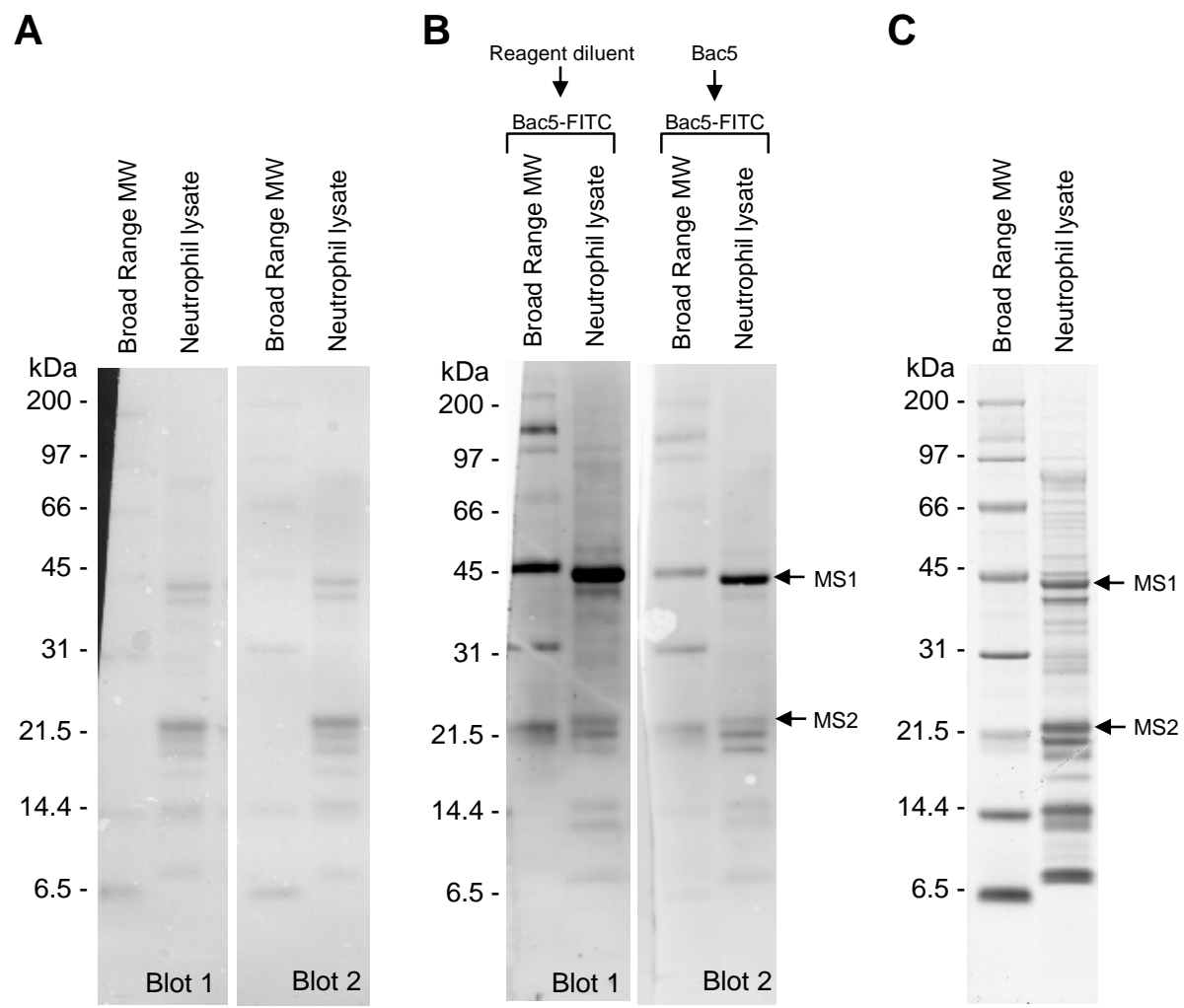

\section{Figure 5.6 Far-western blot of neutrophil lysate}

Untreated naïve neutrophils were lysed in the presence of protease inhibitors. The protein was separated by electrophoresis and analysed by far-western blot using Bac5-FITC. (A) Ponceau S stain for protein loading. (B) Blot 1 was incubated with reagent diluent for 2 hours, washed and probed with Bac5-FITC. Blot 2 was probed with a five-fold excess of unlabelled Bac5 for 2 hours, washed and probed with Bac5-FITC. (C) CBB G-250 stain for LC-MS/MS analysis. MS1 and MS2 indicate bands removed for LC-MS/MS analysis.

Table 5.1 Summary of the LC-MS/MS data for the Bac5-FITC far-western blot

\begin{tabular}{|c|c|c|c|c|c|c|}
\hline Sample & Protein & Peptides & $\begin{array}{c}\text { MOWSE } \\
\text { score }\end{array}$ & $\begin{array}{c}\text { Accession } \\
\text { number }\end{array}$ & Activity & $\begin{array}{c}\text { MW } \\
(\mathrm{kDa})\end{array}$ \\
\hline \multirow[t]{4}{*}{ MS1 } & Beta actin & 26 & 1762 & gi|313507212 & Cell motility & 41.7 \\
\hline & Serpin B1 & 23 & 1483.9 & gi|331028727 & $\begin{array}{l}\text { Regulates neutrophil } \\
\text { protease activity }\end{array}$ & 42.2 \\
\hline & $\begin{array}{l}\text { Serpin B3 } \\
\text { (predicted) }\end{array}$ & 6 & 368.5 & gi|119916473 & $\begin{array}{l}\text { Regulates neutrophil } \\
\text { protease activity }\end{array}$ & 44 \\
\hline & Haptoglobin & 6 & 240.9 & gi|94966763 & Acute-phase protein & 44.9 \\
\hline \multirow[t]{6}{*}{ MS2 } & Serpin B1 & 24 & 1581.1 & gi|331028727 & $\begin{array}{l}\text { Regulates neutrophil } \\
\text { protease activity }\end{array}$ & 42.2 \\
\hline & Beta actin & 23 & 1804.2 & gi|313507212 & Cell motility & 41.7 \\
\hline & S100 A9 & 17 & 1290.2 & gi|296489689 & $\begin{array}{l}\text { Regulation of inflammatory } \\
\text { and immune responses }\end{array}$ & 17.1 \\
\hline & Haptoglobin & 6 & 286.9 & gi|94966763 & Acute-phase protein & 44.9 \\
\hline & Serpin B4 & 5 & 345.1 & gi|151557009 & $\begin{array}{l}\text { Regulates neutrophil } \\
\text { protease activity }\end{array}$ & 44.4 \\
\hline & $\begin{array}{l}\text { Peptidoglycan } \\
\text { recognition protein } 1\end{array}$ & 3 & 143.4 & gi|223635600 & $\begin{array}{l}\text { Gram-positive bactericidal } \\
\text { protein }\end{array}$ & 21 \\
\hline
\end{tabular}


Sample MS1 produced multiple peptides indicating the presence of serpin B1, serpin B3, haptoglobin and $\beta$-actin, which all had predicted molecular weights of the correct size for the excised band [91]. In sample MS2, serpin B1, haptoglobin and $\beta$-actin were also identified, as well as serpin B4. These peptides are likely to be contaminants from the first sample that were inadvertently retained by the LC column during analysis of MS1. Serpin B1 and serpin B3 are cytoplasmic serine protease inhibitors [253] which have additional roles in inflammation, complement activation, apoptosis, and host defence during microbial infection [254]. Considering these roles, and the effects of Bac5 on neutrophils, the potential interaction of Bac5 and serpins warrants further investigation. However, Bac5 was able to modulate migration, ROS production and phagocytosis, suggesting a target protein other than the serpins may be involved.

Haptoglobin is a hepatocyte derived plasma protein involved in the acute phase response but is also synthesised and stored in the secondary granules of neutrophils [255]. Haptoglobin primarily serves in the removal of free haemoglobin and can thereby reduce ROS production [256]. $\beta$-actin is a structural protein involved in neutrophil motility [257,258], degranulation [43], phagocytosis [259] and the regulation of NADPH oxidase activity [260,261]. In sample MS2, multiple peptides were identified for S100A9, a cytosolic $17.1 \mathrm{kDa}$ acute-phase protein [262] which enhances neutrophil migration and phagocytosis, and along with S100A8 (collectively called calprotectin), induces ROS production via transfer of arachidonic acid and via direct interaction with NADPH oxidase subunits $\mathrm{p} 67^{\text {phox }}$ and Rac [263].

Taken together, haptoglobin, $\beta$-actin and S100A9 each have a role in modulation of ROS production. Bac5 may interact with all three of these proteins to induce ROS production. However, the high peptide sequence coverage and MOWSE score in the LC-MS/MS analysis, as well as the strong localisation of Bac5-FITC signal in the farwestern blot indicated $\beta$-actin was the best candidate protein for interaction with Bac5. Therefore $\beta$-actin was investigated in further detail. Nevertheless, the potential for Bac5 to interact with haptoglobin and S100A9 warrants future investigation. 


\subsubsection{Confirmation of the Bac5- $\beta$-actin interaction}

\subsubsection{Bac5 binds immunopurified cellular $\beta$-actin}

Of the proteins identified by MS, $\beta$-actin was considered the most likely candidate for mediating Bac5 ROS production. To confirm the Bac5- $\beta$-actin interaction, $\beta$-actin was purified from neutrophil lysate by immunopurification, and duplicate purifications were separated by SDS-PAGE. One duplicate was stained with CBB R-250 (Figure 5.7 A) which indicated a significant amount of $\beta$-actin was obtained from the purification procedure. The second duplicate was transferred to PVDF membrane and stained with Ponceau S (Figure 5.7 B) which indicated a successful transfer of $\beta$-actin to the membrane. Using Bac5-FITC to probe the membrane (Figure 5.7 C), a distinct FITC signal was obtained corresponding to $\beta$-actin in the lysate start material, the supernatant, and in the immunopurified protein sample, confirming the specificity of Bac5 for $\beta$-actin. 
A

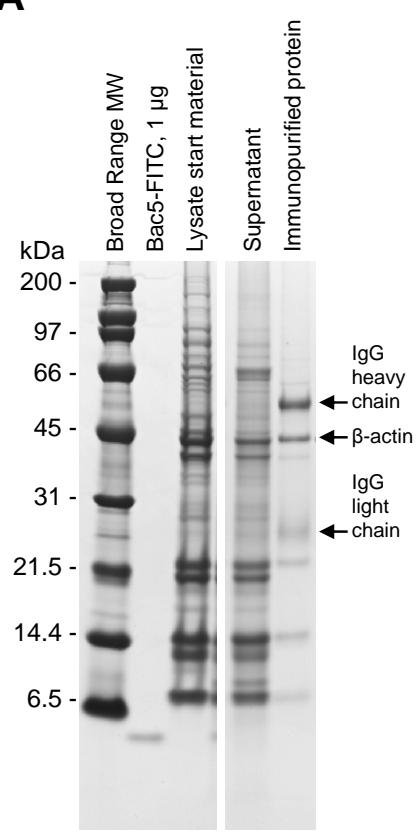

B

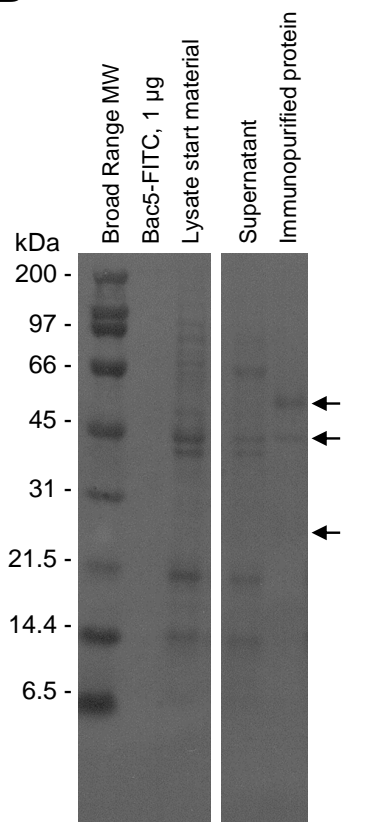

C

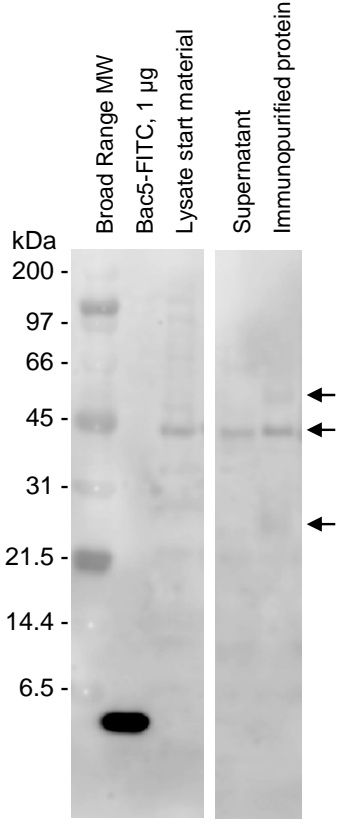

\section{Figure 5.7 Immunopurification of cellular $\beta$-actin and far-western blot}

Neutrophil lysate was mixed with $1 \mu \mathrm{g}$ rabbit anti-actin $\mathrm{IgG}$ antibody overnight and precipitated with protein A-conjugated sepharose beads for two hours. Beads were isolated by centrifugation and prepared for SDS-PAGE and far-western blot using Bac5-FITC as the probe. (A) CBB R-250 stain. (B) Ponceau S stain. (C) The PVDF membrane was probed by far-western blot using $0.5 \mu \mathrm{M}$ Bac5-FITC. Arrows from top to bottom indicate IgG heavy chain, $\beta$-actin and $\operatorname{IgG}$ light chain. 


\subsubsection{Bac5 does not readily co-purify with cellular $\beta$-actin}

The far-western blot technique identifies interactions between a denatured protein immobilised on a membrane, and a native protein used as the probe. To determine if the interaction between Bac5 and $\beta$-actin occurred when $\beta$-actin was in the native folded state, a co-immunoprecipitation procedure was used. Exogenous Bac5-FITC was added to the neutrophil lysate prior to the purification procedure. Parallel samples were also processed in which the neutrophil lysate, purification antibody or exogenous Bac5-FITC were omitted. This allowed non-specific interactions between Bac5-FITC and antibody, or Bac5-FITC and Protein A agarose beads to be measured.

The electrophoresed samples were stained with CBB R-250 (Figure 5.8 A) after a FITC fluorescence image was taken (Figure 5.8 B). A successful immunopurification occurred either in the presence or absence of exogenous Bac5-FITC (Figure 5.8 A, lanes 5 and 11, respectively). However, omission of either the lysate or the antibody resulted in an unsuccessful immunopurification of $\beta$-actin, as expected (Figure $5.8 \mathrm{~A}$, lanes 7 and 9, respectively). The fluorescence image of co-immunopurified Bac5-FITC showed that some Bac5-FITC was associated with each co-immunopurification except where Bac5-FITC was omitted (Figure 5.8 B, lanes 10 and 11). This indicated that Bac5-FITC was non-specifically binding to the antibody or the purification beads, as well as $\beta$-actin. Semi-quantitative analysis allowed the relative density of co-immunopurified Bac5-FITC in each sample to be measured (Figure 5.8 C). Due to the omission of either lysate or antibody, the amount of non-specific binding of Bac5-FITC to antibody, or of Bac5-FITC to the purification beads, could be taken into account. These allowed estimates to be calculated for the proportion of Bac5-FITC bound to each component of the standard co-immunopurification procedure (where no component was omitted) (Figure $5.8 \mathrm{D}$ ). These estimates were: $14.85 \pm 1.89 \%$ of Bac5 bound to native $\beta$-actin, $29.30 \pm 11.31 \%$ bound to antibody, and $55.85 \pm 9.55 \%$ bound to the purification beads. This is assuming that the pre-clearance step (Chapter 2, section 2.7.6) removed any nonspecific binding of protein in the neutrophil lysate.

The occurrence of cathelicidin AMPs binding to antibody was encountered in Chapter 4 (section 4.3.5) and is discussed in Appendix 1. Binding of Bac5-FITC to the agarose purification beads was unexpected. However, the beads are conjugated to protein A, a 
secreted and membrane associated $42 \mathrm{kDa} S$. aureus cell wall protein with a low $\mathrm{pI}$ [264]. Due to the opposing high pI of Bac5, electrostatic interactions probably occur between the protein and the peptide resulting in non-specific binding.

A

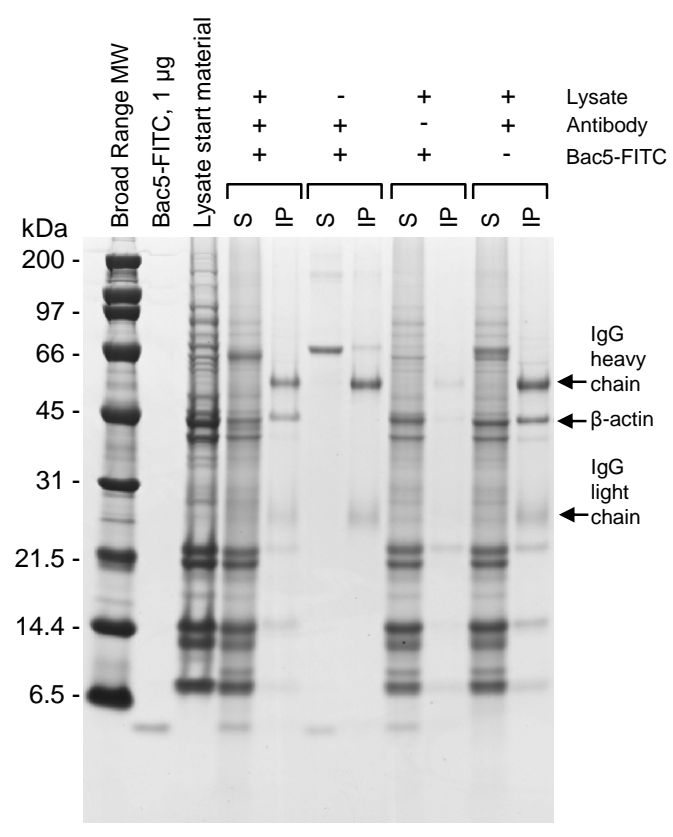

B

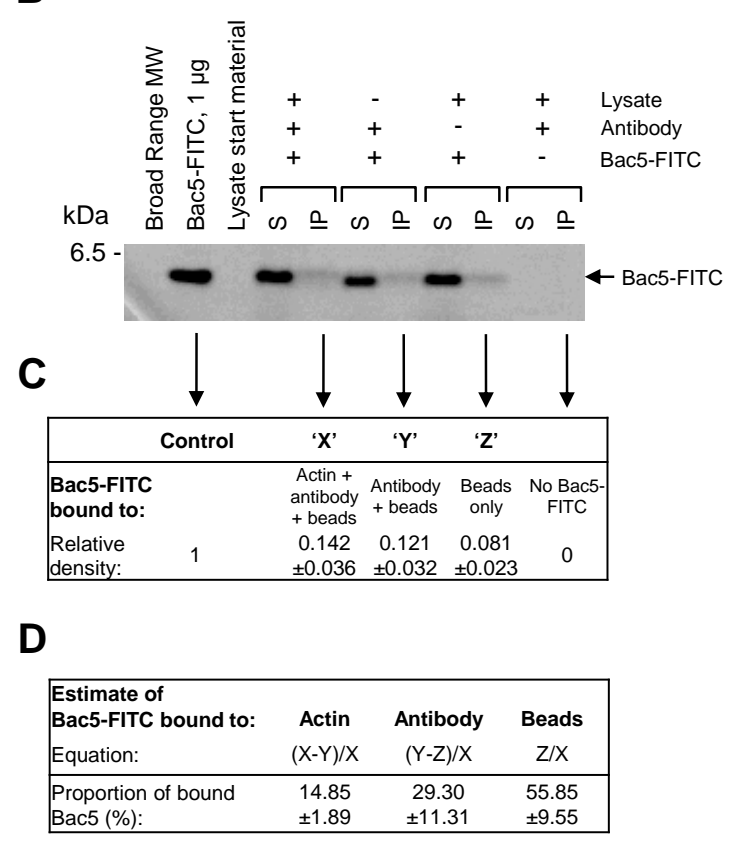

\section{Figure 5.8 Co-immunopurification of cellular $\beta$-actin and Bac5}

Neutrophils were treated with protease inhibitors before lysis. As indicated, $800 \mu \mathrm{g}$ lysate was mixed with $1 \mu \mathrm{g}$ rabbit anti-actin IgG antibody and $10 \mu \mathrm{g}$ Bac5-FITC overnight. Bac5-FITC- $\beta$-actin complexes were precipitated with protein A-conjugated sepharose beads for 2 hours. The sepharose beads were isolated by centrifugation and prepared for SDS-PAGE analysis along with supernatant samples. (A) CBB R-250 stain. (B) Molecular Imager FX scan at $488 \mathrm{~nm}$ excitation and $540 \mathrm{~nm}$ emission for FITC fluorescence (495 nm excitation, $519 \mathrm{~nm}$ peak emission). (C) Semi-quantitative analysis of co-immunopurified Bac5FITC. (D) Calculation of the estimated proportions of Bac5-FITC binding to immunopurification components. Data represent the mean \pm SEM for three independent experiments. Normalisation to control and calculations were performed for each experiment before calculating means. S, supernatant sample; IP, immunopurified protein. 


\subsubsection{Co-immunopurification of Bac5 and purified $\beta$-actin}

To investigate whether endogenous protein in the neutrophil lysate was potentially altering the Bac5- $\beta$-actin interaction, an identical co-immunopurification procedure was performed with purified non-muscle actin in place of the neutrophil lysate. As in the previous section, the electrophoresed samples were stained with CBB R-250 (Figure 5.9) after a FITC fluorescence image was taken (Figure 5.9 B).

Successful immunopurifications occurred in either the presence or absence of exogenous Bac5-FITC (Figure 5.9 A, lanes 5 and 11, respectively). As expected, omission of purified $\beta$-actin resulted in an unsuccessful immunopurification (Figure $5.9 \mathrm{~A}$, lane 7). However, omission of the antibody still resulted in some $\beta$-actin being purified (Figure 5.9 A, lane 9). This could be explained by the fact that Bac5-FITC binds both $\beta$-actin and the purification beads, and in this case may be acting in a similar manner to the antibody. This would also explain why more $\beta$-actin was immunopurified in the presence of Bac5 compared to when Bac5 was omitted (Figure 5.9 A, lanes 5 and 11). The presence of excess protein in the neutrophil lysate co-immunopurification potentially acted to block this effect. This anomaly subsequently affected the co-immunopurification analysis (Figure 5.9 B, C) which may have contributed to the large errors in the estimates of Bac5-FITC binding in the standard coimmunopurification procedure (Figure 5.9 D). These estimates were $21.55 \pm 23.27 \%$ for Bac5-FITC bound to $\beta$-actin, $34.73 \pm 32.98 \%$ bound to antibody and $43.72 \pm 15.04 \%$ bound to the purification beads. 
A

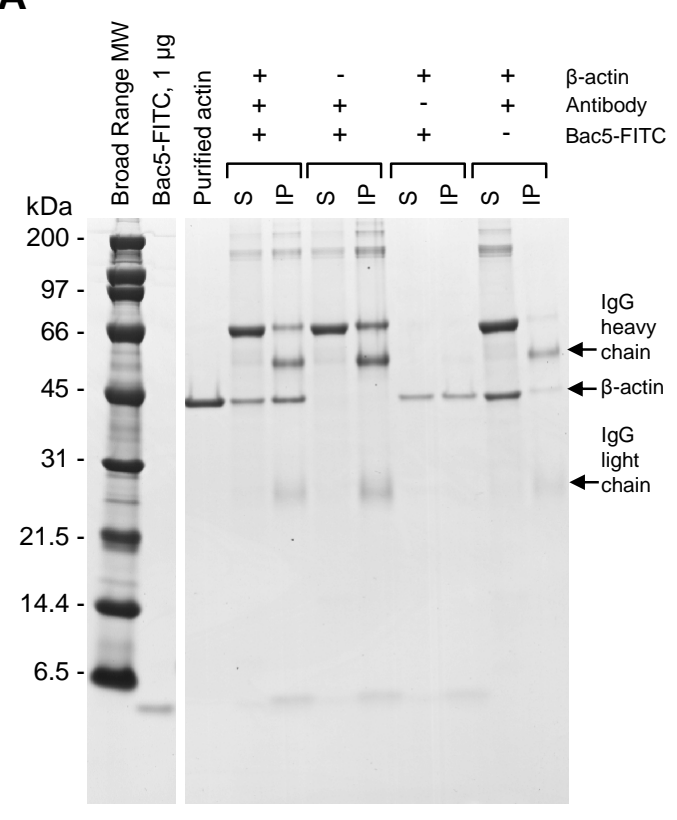

B

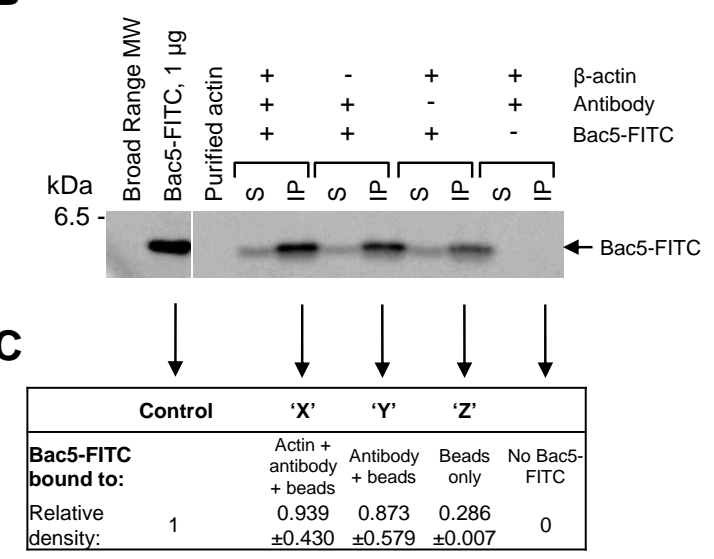

D

\begin{tabular}{|lccc|}
\hline Estimate of & Actin & Antibody & Beads \\
Bac5-FITC bound to: & $(\mathrm{X}-\mathrm{Y}) / \mathrm{X}$ & $(\mathrm{Y}-\mathrm{Z}) / \mathrm{X}$ & $\mathrm{Z} / \mathrm{X}$ \\
Equation: & 21.55 & 34.73 & 43.72 \\
\hline Proportion of bound & \pm 23.27 & \pm 32.98 & \pm 15.04 \\
\hline Bac5 (\%): &
\end{tabular}

\section{Figure 5.9 Co-immunopurification of purified $\beta$-actin and Bac5}

As indicated, $1 \mu \mathrm{g}$ purified non-muscle actin was mixed with $1 \mu \mathrm{g}$ rabbit anti-actin IgG antibody and $10 \mu \mathrm{g}$ Bac5-FITC overnight. Bac5-FITC- $\beta$-actin complexes were precipitated with protein A-conjugated sepharose beads for 2 hours. The beads were isolated by centrifugation and prepared for SDS-PAGE analysis along with TCA precipitated supernatant samples. (A) CBB R-250 stain. (B) Molecular Imager FX scan at $488 \mathrm{~nm}$ excitation and $540 \mathrm{~nm}$ emission. (C) Semi-quantitative analysis of co-immunopurified Bac5-FITC. (D) Calculation of estimated proportions of Bac5-FITC binding to immunopurification components. Data represent the mean \pm SEM for three independent experiments. Normalisation to control and calculations were performed for each experiment before calculating means. S, supernatant sample; IP, immunopurified protein. 


\subsubsection{Bac5 colocalises with cytoplasmic $\beta$-actin}

Confocal microscopy was used to assess the cellular localisation of Bac5-FITC and $\beta$-actin. Naïve neutrophils were treated with $2.5 \mu \mathrm{M}$ Bac5-FITC for 60 minutes, washed extensively to remove extracellular Bac5-FITC, fixed, and stained with the polymerised actin-specific fluorescent conjugate phalloidin-TRITC (Figure 5.10) [265]. The phalloidin-TRITC signal was consistently strong at the membrane and within the cytoplasm of both Bac5-FITC-treated and untreated neutrophils. In treated neutrophils the Bac5-FITC signal was consistently stronger in the cytoplasm than at the membrane, and consistently co-localised with cytoplasmic rather than membranous actin.

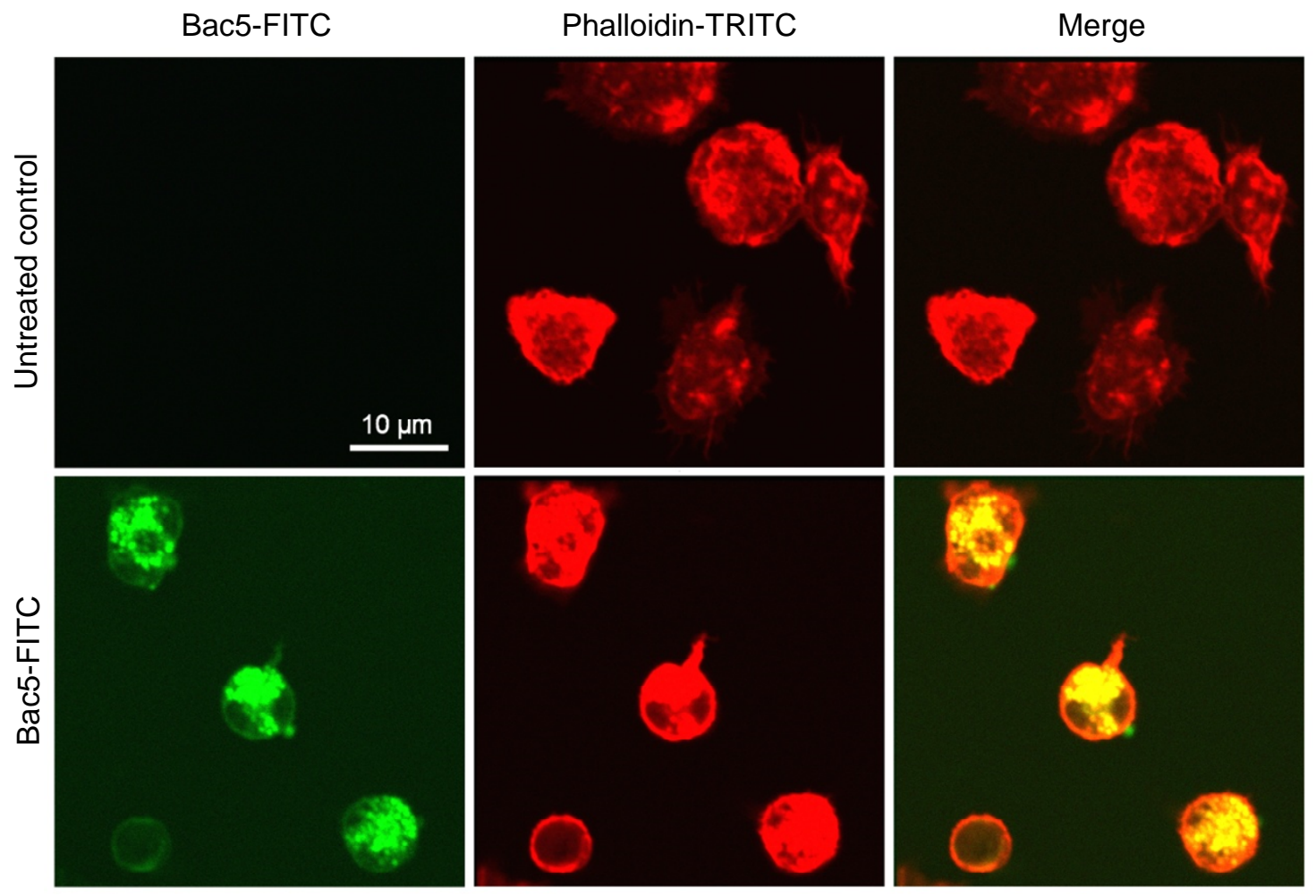

Figure 5.10 Confocal microscopy of Bac5 and $\beta$-actin colocalisation

Neutrophils were seeded onto glass coverslips and incubated with $2.5 \mu \mathrm{M}$ Bac5-FITC for 60 minutes. Cells were washed extensively to remove excess Bac5-FITC, fixed, permeabilised, blocked and incubated with phalloidin-TRITC $(5 \mu \mathrm{g} / \mathrm{ml})$ for 1 hour. Images are representative of two independent experiments. 


\subsubsection{Bac5, intracellular ROS and $\beta$-actin have a cytoplasmic association}

Confocal microscopy was used to assess the cellular localisation of Bac5-FITC, intracellular ROS and $\beta$-actin. Naïve neutrophils were treated with $2.5 \mu \mathrm{M}$ Bac5-FITC and CellROX Deep Red for 60 minutes, washed extensively to remove extracellular Bac5-FITC, fixed, and stained with the actin-specific fluorescent conjugate phalloidin-TRITC (Figure 5.11). The untreated controls showed minor intracellular ROS production in the absence of Bac5, which was confined to the cytoplasm. As seen in previous figures (Figure 5.4 and Figure 5.10), the strong cytoplasmic Bac5-FITC signal colocalises with both the CellROX Deep Red signal and the phalloidin-TRITC signal. This indicates that Bac5 binds to actin structures as phalloidin only binds filamentous actin, not free actin. At the membrane the CellROX Deep Red signal was virtually absent while the Bac5-FITC signal was present, albeit at a less intense level. This implies that a Bac5- $\beta$-actin interaction at the membrane does not stimulate intracellular ROS production, however this observation may account for extracellular ROS production observed in Chapter 4 (see Section 4.3.3.4 and Figure 4.8).

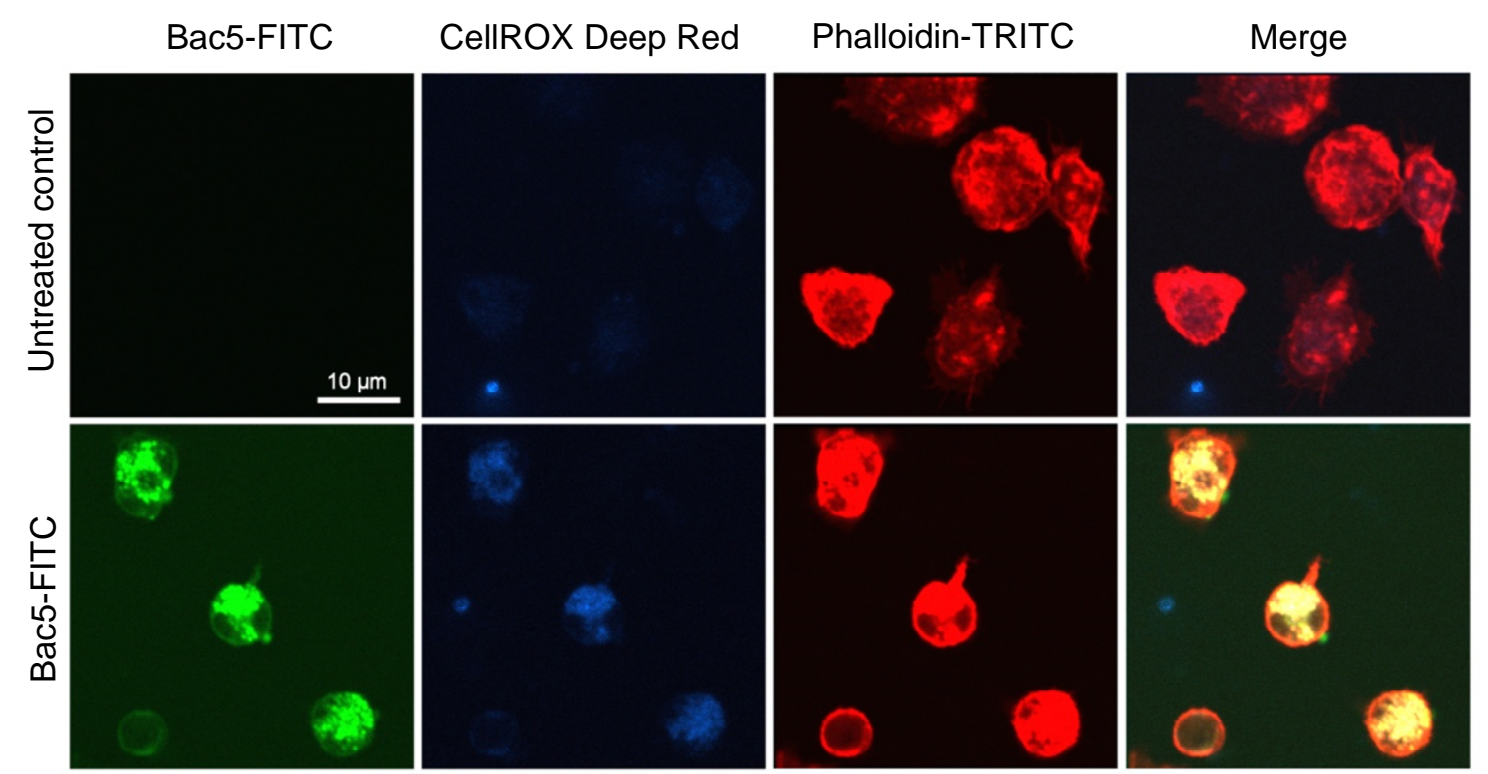

Figure 5.11 Confocal microscopy of Bac5, intracellular ROS and $\beta$-actin colocalisation

Neutrophils were seeded onto glass coverslips and incubated with $2.5 \mu \mathrm{M}$ Bac5-FITC and CellROX Deep Red for 60 minutes. Cells were washed extensively to remove excess Bac5-FITC, fixed, permeabilised, blocked and incubated with phalloidin-TRITC $(5 \mu \mathrm{g} / \mathrm{ml})$ for 1 hour. Images are representative of two independent experiments. 


\subsubsection{Activated neutrophils}

As Bac5 was able to inhibit PMA-induced extracellular ROS production (see Chapter 4, section 4.3.3.4), the effect of Bac5 on intracellular ROS production in PMA-activated neutrophils was assessed, as well as the cellular localisation of Bac5 in activated cells.

\subsubsection{Intracellular ROS is not inhibited by Bac5}

To assess the effect of Bac5 on intracellular ROS in activated cells, the fluorescent ROS indicator CellROX Deep Red was used as in section 5.3.2. Bac5-FITC was also used to determine whether higher concentrations of Bac5 were able to inhibit intracellular ROS production in a similar manner to the inhibition of extracellular ROS production observed in Chapter 4. The activated cells were analysed by flow cytometry, and confocal microscopy was used to assess the cellular localisation of Bac5 and intracellular ROS production.

The flow cytometry gating strategy followed that used in section 5.3.2 and the data were analysed using CellROX Deep Red histograms of Bac5-FITC-positive cells (Figure 5.12 A). After 30 minutes a trend toward inhibiting PMA-induced ROS production at higher concentrations of Bac5 was observed, however intracellular ROS was still significantly greater than the activated control (Figure 5.12 B). After 60 minutes this effect was no longer apparent, and intracellular ROS production increased in a dose-dependent manner.

As Bac5 regulated extracellular PMA-induced ROS production, intracellular ROS production in naïve cells and activated cells was compared to determine if Bac5 also had a regulatory effect. After 30 minutes activated cells produced significantly more ROS at low Bac5 concentrations however ROS production was equivalent in naïve and activated cells at high Bac5 concentrations (Figure 5.12 C). After 60 minutes naïve and activated cells produced equivalent intracellular ROS at all Bac5 concentrations tested (Figure 5.12 D). This indicated that Bac5 does not regulate PMA-induced intracellular ROS. In fact, PMA has virtually no effect on intracellular ROS production. Instead Bac5 was the main determinant of the intracellular ROS response. In summary, the effect of Bac5 on extracellular ROS production is dependent on the activation state of 
the neutrophils, whereas the effect of Bac5 on intracellular ROS production is independent of the activation state of neutrophils.

A
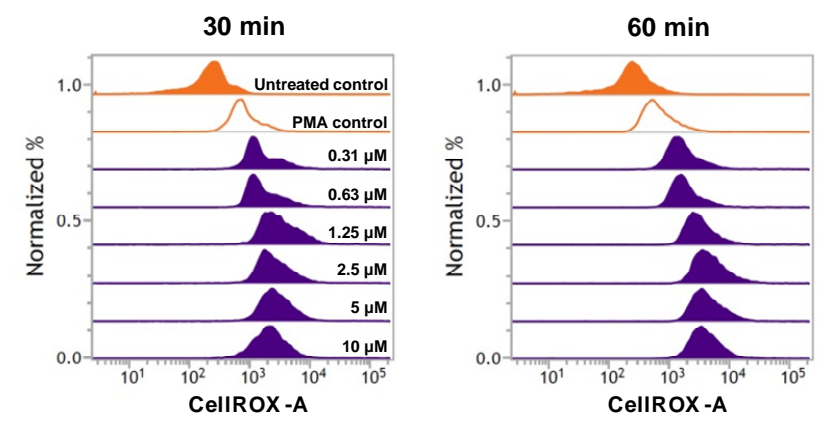

B

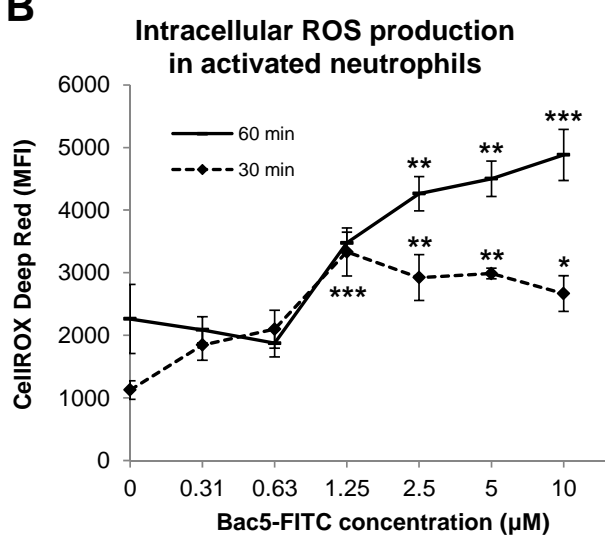

C

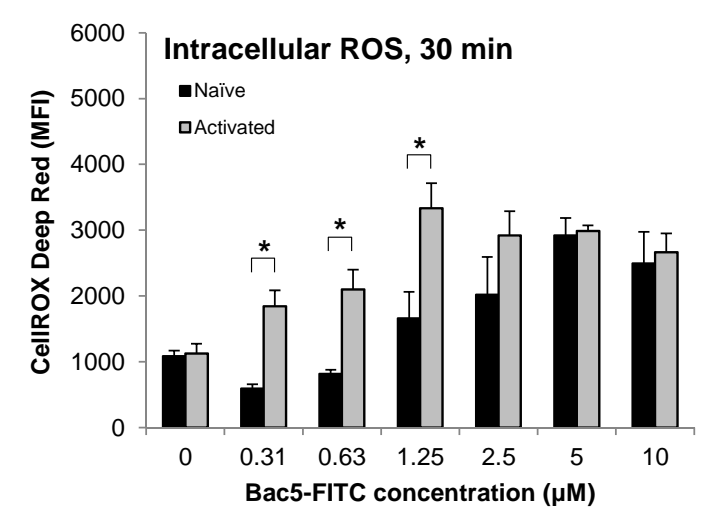

D

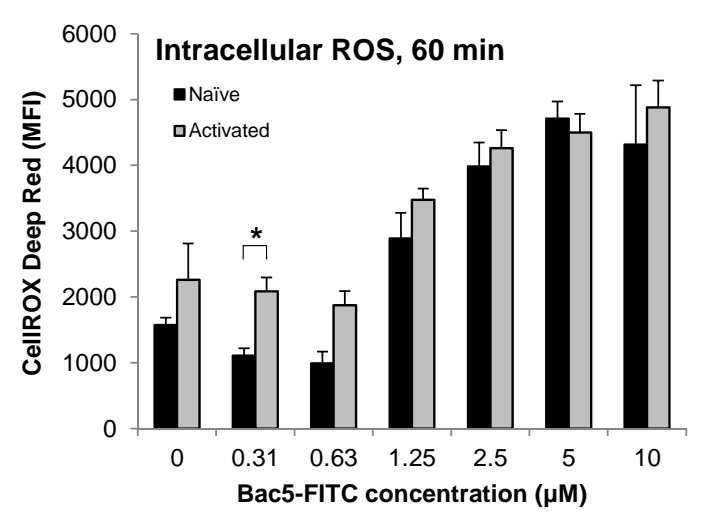

Figure 5.12 Flow cytometry analysis of PMA-induced intracellular ROS production in the presence of Bac5

The fluorescent intracellular ROS indicator CellROX Deep Red was used to measure intracellular ROS induced by $100 \mathrm{ng} / \mathrm{ml}$ PMA in the presence of Bac5-FITC using flow cytometry. (A) Stacked histograms of Bac5-FITC-positive/CellROX-positive neutrophils at 30 and 60 minutes. (B) Dose-response curves for intracellular ROS production in activated cells in the presence of Bac5-FITC. Intracellular ROS production in naïve and activated neutrophils was compared after (C) 30 minutes and (D) 60 minutes of Bac5-FITC treatment. Data represent the mean \pm SEM for three independent experiments performed in duplicate. Statistical significance was determined by one-way ANOVA with Tukey's post-test comparing treatments with control. * $\mathrm{P}<0.05, * * \mathrm{P}<0.01, * * * \mathrm{P}<0.001$. 


\subsubsection{Bac5 prevents $\mathrm{p} 47^{\text {phox }}$ translocation in activated neutrophils}

Extracellular ROS production by activated neutrophils was significantly inhibited by $10 \mu \mathrm{M}$ Bac5 (Chapter 4, section 4.3.3.4). This concentration was used to inhibit PMAinduced ROS production for 30 or 60 minutes. The neutrophils were then prepared as in section 5.3.4.1. Data were adjusted for protein loading (Figure $5.13 \mathrm{~A}$ ) and $\mathrm{p} 47^{\mathrm{phox}}$ signal (Figure 5.13 B). A trend toward increased translocation to the membrane was observed in the PMA-treated controls (Figure $5.13 \mathrm{C}$ ). In PMA-activated cells treated with Bac5 there was no trend toward translocation of $\mathrm{p} 47^{\text {phox }}$ at 30 or 60 minutes, indicating Bac5 prevents the translocation of $\mathrm{p} 47^{\text {phox }}$.

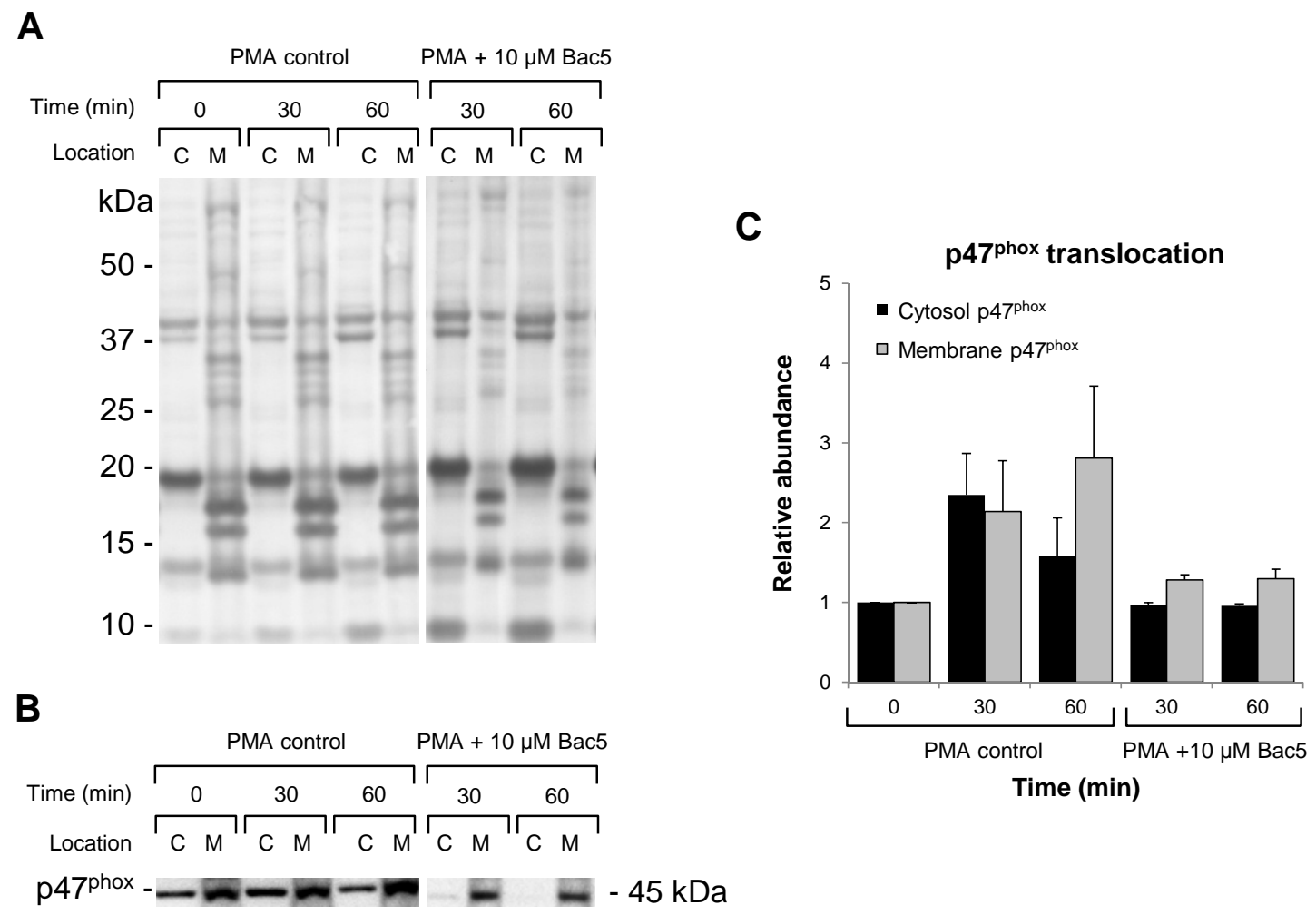

Figure 5.13 Western blot analysis of $\mathrm{p}^{\mathrm{phox}}$ translocation in activated cells

Neutrophils were activated with $100 \mathrm{ng} / \mathrm{ml}$ PMA and treated with $10 \mu \mathrm{M}$ Bac5 for 30 or 60 minutes. Cells were lysed, separated into cytosol and membrane fractions and $30 \mu \mathrm{g}$ protein probed by western blot using anti-p47 $7^{\text {phox }}$ antibody. (A) Representative Ponceau S stain for loading controls. (B) Representative anti-p4 $7^{\text {phox }}$ antibody probe. (C) Semi-quantitative analysis of the relative abundance of $447^{\text {phox }}$ in cytosol and membrane fractions. Data represent the mean \pm SEM for three independent experiments. No statistical significance was found using a one-way ANOVA and Tukey's post-test. C, cytosol; M, membrane. 


\subsubsection{Colocalisation of Bac5, intracellular ROS and $\beta$-actin is unchanged in activated cells}

Confocal microscopy was used to assess the cellular localisation of Bac5-FITC, intracellular ROS and $\beta$-actin in activated neutrophils. Cells were activated with PMA and were treated with $10 \mu \mathrm{M}$ Bac5-FITC and CellROX Deep Red for 60 minutes, washed extensively to remove extracellular Bac5-FITC, fixed, and stained with the actin-specific fluorescent conjugate phalloidin-TRITC (Figure 5.14). The PMA-treated control showed significant CellROX Deep Red signal, although this signal was less intense than in Bac5-treated cells. As in naïve cells, activated cells produced intracellular ROS in a diffuse pattern throughout the cytoplasm, and the distribution of Bac5-FITC also colocalised with intracellular ROS in activated cells. These signals also strongly localised with the phalloidin-TRITC signal, indicating that as in naïve cells, Bac5 associates with actin to regulate intracellular ROS production in activated cells. The phalloidin-TRITC signal at the membrane was also less intense in Bac5-treated neutrophils than in PMA-treated controls, indicating association of actin with the membrane may be lost in activated cells treated with Bac5.

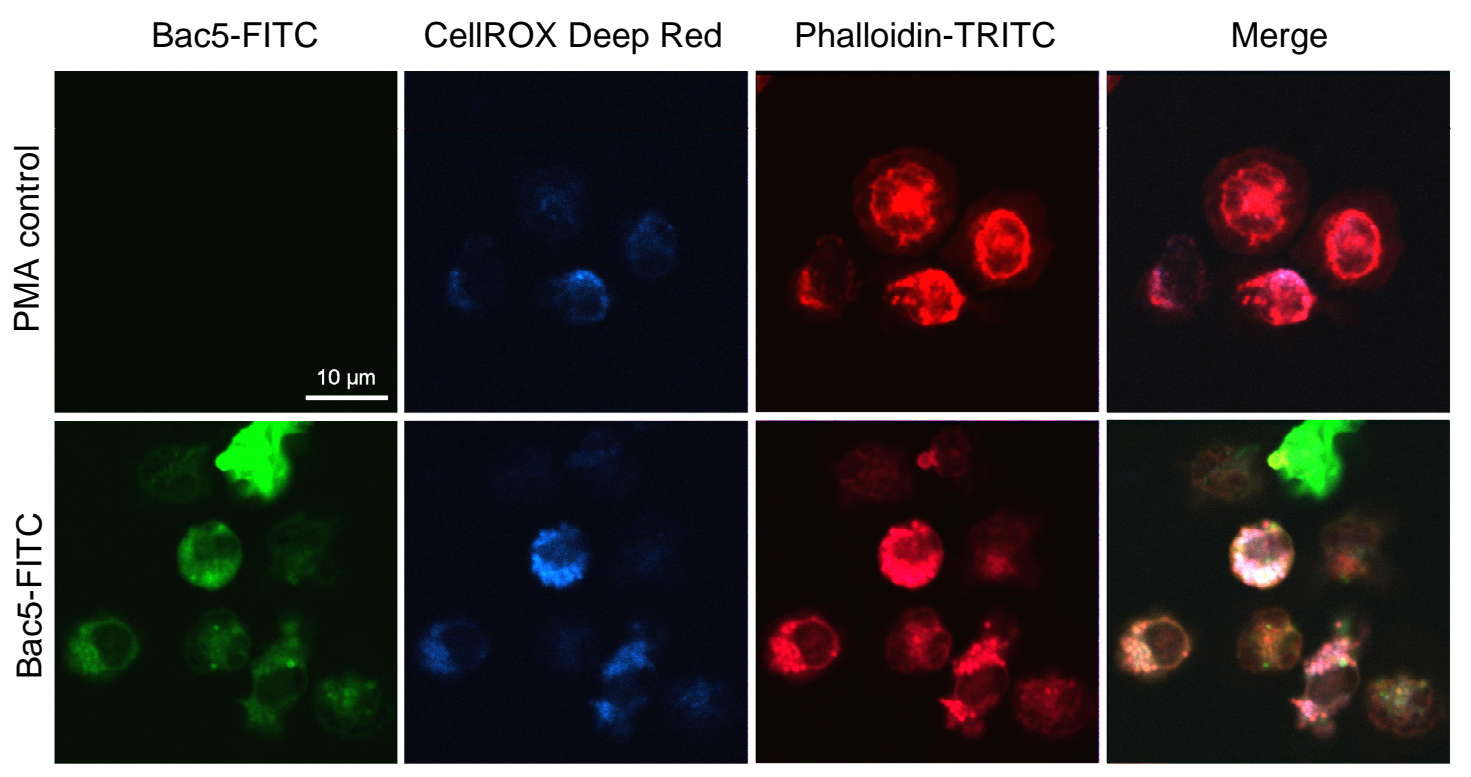

Figure 5.14 Confocal microscopy of Bac5, intracellular ROS and $\beta$-actin

Neutrophils were seeded onto glass coverslips, activated with $100 \mathrm{ng} / \mathrm{ml}$ PMA and incubated with $10 \mu \mathrm{M}$ Bac5-FITC and CellROX Deep Red for 60 minutes. Cells were washed extensively to remove excess Bac5-FITC, fixed, permeabilised, blocked and incubated with phalloidin-TRITC ( $5 \mu \mathrm{g} / \mathrm{ml})$ for 1 hour. Images are representative of two independent experiments. 


\subsection{Discussion}

The aims of the experiments in this chapter were to determine whether Bac5 was internalised by neutrophils, and to elucidate the mechanism by which Bac5 modulates neutrophil function.

Previously, FITC conjugates of bovine Bac5 and Bac7 have been reported to be rapidly internalised into non-bovine cell lines such as murine NIH 3T3 fibroblasts and human U937 lymphoma cells [247,248]. This occurs via both macropinocytosis and direct membrane translocation [248]. Once internalised, Bac5 and Bac7 were shown to localise to nucleoli and nuclear structures, and in fibroblasts initiate DNA synthesis and proliferation. The experiments in this chapter demonstrated that Bac5 was internalised by bovine neutrophils. Confocal microscopy showed the presence of both a diffuse and vesicle-like localisation of Bac5, indicating direct membrane translocation and active internalisation, respectively. However, in contrast to internalisation in other cell lines, Bac5 remained localised to the cytoplasm of neutrophils and did not appear to enter the nucleus. This suggested a different mode of action in immune cells, which corresponds with the observed modulation of neutrophil innate immune function seen in Chapter 4, rather than longer-term regulation of gene expression or cell proliferation as seen in other cell types.

Internalisation of Bac5 was associated with intracellular ROS production. This appeared to occur within the cytoplasm and in intracellular vesicles. Interestingly, both naïve and activated neutrophils showed similar patterns of intracellular ROS production in the presence of Bac5, while cells treated with PMA alone only showed limited intracellular ROS. This indicated that Bac5 was the dominant agonist inducing intracellular ROS production. The results of Chapter 4 showed that PMA alone induced a huge increase in extracellular ROS, but in the presence of high concentrations of Bac5 the response was significantly inhibited. Taken with the results from Chapter 4, the role of Bac5 in regard to ROS production appears to be regulatory in which global ROS production, i.e. both intracellular and extracellular ROS production, is maintained at a relatively low but significant level independent of the activation status of the neutrophil. 
The aims of the remaining experiments in this chapter were to elucidate the mechanism by which Bac5 was able to modulate neutrophil function, in particular the regulation of ROS production. To this end, the translocation of the $\mathrm{p} 47^{\text {phox }} \mathrm{NADPH}$ oxidase subunit was assessed in naïve and activated neutrophils in the presence of Bac5 to determine if Bac5 upregulated NADPH oxidase activity at the membrane.

The results showed $\mathrm{p} 47^{\text {phox }}$ was not translocated to the membrane in Bac5-treated naïve neutrophils, indicating $\mathrm{p} 47^{\text {phox }}$ is not required for Bac5-induced ROS production. As $\mathrm{p} 47^{\text {phox }}$ is required for agonist-induced (e.g. PMA) ROS production, but not basal ROS production [251], it appears that rather than fully activating the oxidase complex, Bac5 may instead only increase basal ROS production in a $447^{\text {phox }}$-independent manner. This theory agrees with the low level of Bac5-induced extracellular and intracellular ROS production, compared to the 16-fold increase in extracellular PMA-induced ROS production. Further to this, $\mathrm{p} 47^{\text {phox }}$ regulates plasma membrane ROS production, but not phagosome ROS production [266]. This explains why intracellular ROS production was virtually absent in PMA-treated controls and why Bac5 was the dominant agonist in both naïve and activated cells.

The results also suggested that $\mathrm{p} 47^{\text {phox }}$ was not translocated in activated cells treated with Bac5, which also exhibited decreased extracellular ROS production (Chapter 4, section 4.3.3.4). These data agree with the requirement of $\mathrm{p} 47^{\text {phox }}$ in PMA-induced ROS production. Therefore it appears that Bac5 blocks translocation of $\mathrm{p} 47^{\text {phox }}$ in PMAtreated cells. Blocking of $\mathrm{p} 47^{\text {phox }}$ by Bac5 may also be part of the mechanism by which Bac5 enhances basal ROS production in naïve cells. Unphosphorylated $\mathrm{p} 47^{\text {phox }}$ inhibits ROS production at the plasma membrane $[267,268]$ thereby maintaining low basal ROS production. The presence of Bac5 may block and remove the inhibitory function of unphosphorylated $\mathrm{p} 47^{\text {phox }}$ leading to enhanced basal ROS production.

Surprisingly, far-western blotting did not identify an interaction of Bac5 with any of the NADPH oxidase components. Instead, Bac5 predominantly bound to $\beta$-actin. $\beta$-actin has an extensive role in cytoskeletal rearrangement, leading to migration, degranulation, phagocytosis and respiratory burst [43,257-261]. These roles are consistent with the modulation of neutrophil functions induced by Bac5. 
Modulation of the actin cytoskeleton is closely involved with NADPH oxidase function [261]. In particular, Rac1, Rac2 and p47 $7^{\text {phox }}$ interact directly with actin [260]. Interestingly, the region of $\mathrm{p} 47^{\text {phox }}$ involved in binding to actin is a highly basic arginine and lysine-rich C-terminal domain called the autoinhibitory region [269]. Although the region of actin to which $\mathrm{p} 47^{\text {phox }}$ binds has not been defined, it is highly probable that Bac5 is able to bind in this same region of actin given the highly basic nature of the peptide. This lends further support to the theory that Bac5 differentially induces and inhibits extracellular ROS production by blocking assembly of $\mathrm{p} 47^{\text {phox }}$ on plasma membrane NADPH oxidase complexes. Competitive far-western blotting using p47 $7^{\text {phox }}$ to probe Bac5- $\beta$-actin complexes would clarify this Bac5-blocking theory.

Although Bac5-induced ROS production at the plasma membrane and within endocytosed vesicles might involve blocking of $\mathrm{p} 47^{\mathrm{phox}}$, it is less clear if and how cytoplasmic ROS is induced by Bac5. Cytoplasmic ROS is generally produced in mitochondria by the electron transport chain during production of adenosine triphosphate [270]. To distinguish cytoplasmic and vesicular ROS, a mitochondrial ROS-specific stain could be employed to clarify whether Bac5 induces cytoplasmic ROS prior to further mechanistic research.

A question remains as to how a Bac5-actin interaction might translate into the other functions that Bac5 modifies. As extensive actin remodelling must occur in order for these functions to be carried out, potential modes of action for Bac5 may include facilitation of $\beta$-actin turnover, activation of actin-binding proteins, or facilitation of actin branching in a similar role to the Arp2/3 complex [258]. Interestingly, the latter complex is activated by Wiskott-Aldrich syndrome protein/suppressor of G-proteincoupled cyclic-AMP receptor (WASp/SCAR) proteins through binding to a C-terminal proline-rich domain [271], similar to the Bac5 sequence. Further research is required to uncover the relationship between Bac5, actin binding and modulation of neutrophil function. 
A

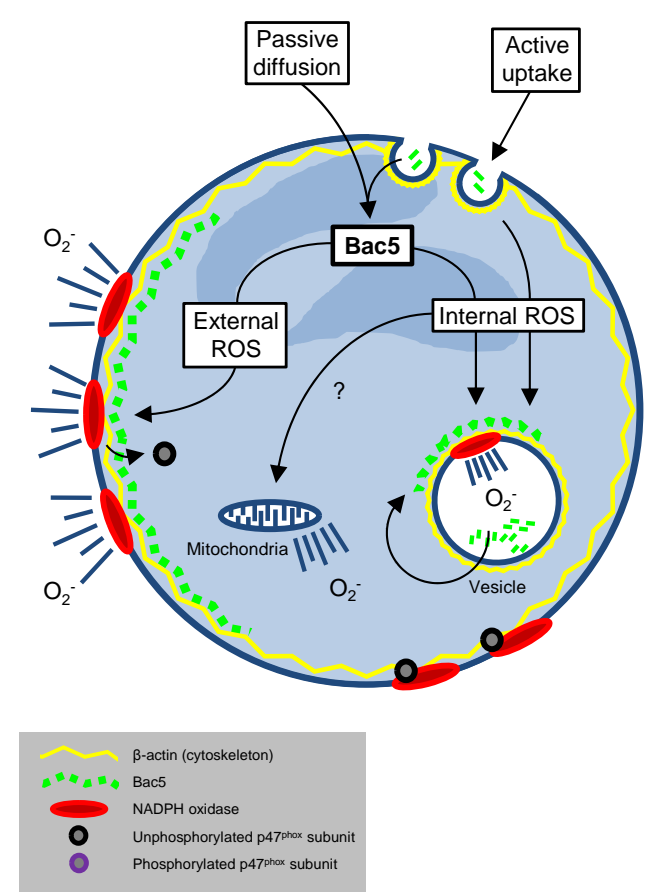

B
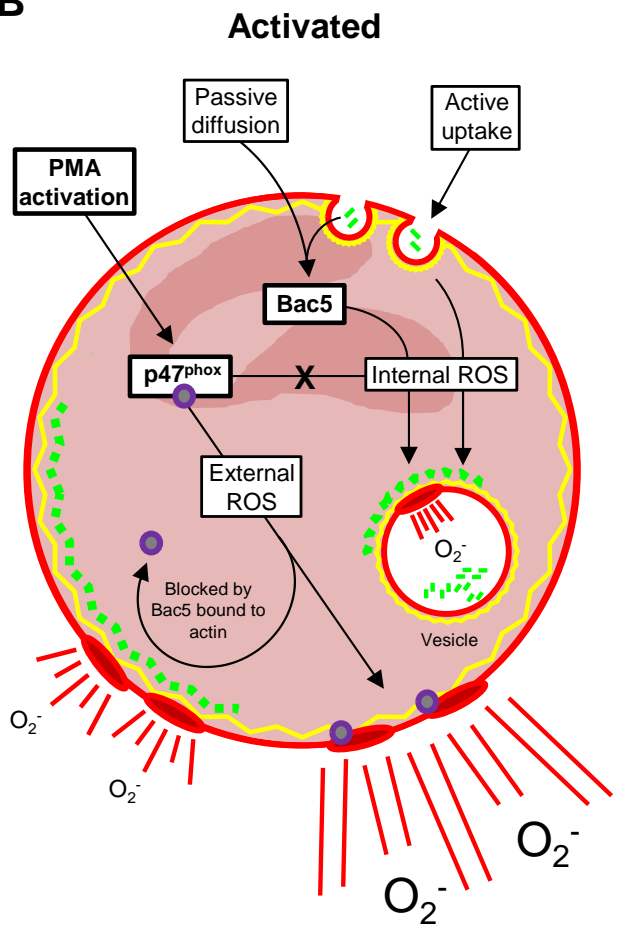

Figure 5.15 Summary of the potential mechanisms of Bac5-induced ROS production in bovine neutrophils

After Bac5 is internalised in naïve neutrophils (A) by passive diffusion or active uptake, the peptide binds to $\beta$-actin at the plasma membrane and enhances basal extracellular ROS production potentially by removing unphosphorylated $\mathrm{p} 47^{\text {phox }}$. Bac5 may also bind to $\beta$-actin around endocytic vesicles to induce internal ROS production. It is unclear whether cytoplasmic ROS are the product of Bac5 interacting with mitochondria. After Bac5 is internalised in PMA-activated neutrophils (B), a similar localisation to $\beta$-actin occurs as in naïve cells. However, this potentially prevents the phosphorylated $\mathrm{p}^{4} 7^{\text {phox }}$ subunit binding to the oxidase complex thereby limiting extracellular ROS production to enhanced basal levels seen in naïve cells. The $\mathrm{p} 47^{\text {phox }}$ subunit does not regulate intracellular ROS therefore intracellular ROS production is induced by Bac5 at similar levels in naïve and activated cells. 


\subsection{Conclusion}

The results of this chapter demonstrate the ability of Bac5 to be internalised by bovine neutrophils and provides an explanation for the differential induction and inhibition of extracellular ROS production through inhibition of $\mathrm{p} 47^{\text {phox }}$ assembly. Although the mechanism of intracellular ROS production could not be determined, the identification of $\beta$-actin as a possible major intracellular target for Bac5 is consistent with the ability of Bac5 to modulate multiple neutrophil functions. Further in vitro studies are required to determine the exact interaction of Bac5 with actin and how this translates into the modulation of neutrophil innate immune functions. 
Chapter 6: General Discussion 


\subsection{Overview}

The cathelicidins are a family of innate immune host defence proteins containing a highly conserved C-terminal CLD and a highly variable AMP domain. The innate immune system of cattle has evolved to express seven isoforms of cathelicidin which vary significantly in their AMP domain. The high conservation of the CLD across species implies an important role in host defence which has not been clearly defined. Likewise, minimal progress has been made toward identifying the immunomodulatory effects of bovine cathelicidin AMPs on host immune cells, despite several advances in other species.

The main aim of this thesis was to elucidate the role of bovine cathelicidins in innate host defence. Based on this aim, unpublished research findings (Food \& Bio-based Products Group, AgResearch), and research from the literature, three hypotheses were tested. Firstly, due to the observed activity of a native cathelicidin-1 preparation, recombinant versions of cathelicidin-1 were hypothesised to have similar humoral activity and cytotoxicity. Secondly, due to the existence of several highly variable AMP domains in bovine, these different AMP domains were hypothesised to modulate bovine neutrophil function in different ways, depending on their structure and neutrophil activation status. Thirdly, as the Bac5 peptide exhibited an ability to regulate neutrophil respiratory burst in the absence of intracellular calcium mobilisation, Bac5 was hypothesised to act via an intracellular mechanism.

Although recombinant versions of the CLD were unable to emulate the activity of native Cath1, the native Cath1 protein was able to bind LPS, agglutinate microbes and induce neutrophil death. The agglutinating activity of the CLD would be complementary to the antimicrobial effects of the AMP domains, while also acting to sequester bacteria and LPS from activating pro-inflammatory responses. This suggests an antimicrobial and anti-inflammatory role for the bovine CLD in host defence. In contrast, the necrosis of neutrophils induced by the CLD could be considered antiinflammatory and counterproductive in the context of host defence. However, excessive neutrophil infiltration and pro-inflammatory responses may cause damage to host tissues in the vicinity of inflammatory foci. Therefore necrosis induced by the CLD may act as a broad regulatory mechanism by controlling unchecked or excessive neutrophil 
activity. Additionally, necrotic neutrophils have been shown to be anti-inflammatory via the release of $\alpha$-defensins [272] and clearance of dead and dying neutrophils via phagocytosis by macrophages is a common route to the resolution of infection [273]. Hence necrosis induced by the CLD and the agglutination of microbes potentially contributes to a broad anti-inflammatory role for the CLD during infection.

The bovine cathelicidin AMPs each had different effects on neutrophil function which in some cases were dependent on the activation status of the neutrophil. Low concentrations of AMP were able to enhance the pro-inflammatory functions of naïve cells or maintain the viability and effectiveness of activated cells. The enhancement of migration is an activity common to human [42,150,162,163], murine [163] and porcine [154] cathelicidins. Induction of ROS production has been observed in human neutrophils treated with LL-37, and suppression of apoptosis has been observed in macrophages treated with porcine PR-39 [185]. This suggests that enhancement of proinflammatory functions at low AMP concentrations may be a common property, independent of structure, of cathelicidin AMPs from different species.

High concentrations of AMP were able to promote phagocytosis but also induced necrosis, particularly in activated cells. Likewise, LL-37 and the structurally distinct defensins have been show to enhance phagocytosis $[82,169,170]$. This may also be a common property of structurally distinct AMPs as the structurally different bovine cathelicidins, namely linear Bac1, Bac5 and BMAP-34, each enhanced phagocytosis. The ability to induce necrosis appears to be a common activity of $\alpha$-helical peptides. BMAP-28 and BMAP-34 each induced neutrophil necrosis, with BMAP-28 demonstrating a distinct membrane permeating effect, as has been observed in other cell lines [157-159]. LL-37 has been shown to induce necrosis in apoptotic neutrophils $[153,172,173]$ which appears to be an anti-inflammatory mechanism promoting clearance of neutrophils. At this stage it is unclear whether necrosis induced by bovine cathelicidins is anti-inflammatory. Nevertheless, high concentrations of cathelicidins appear to regulate proinflammatory neutrophil functions and neutrophil abundance during infection. 
The regulatory effect of Bac5 on extracellular neutrophil respiratory burst was found to involve an interaction with $\beta$-actin. Low concentrations of Bac5 enhanced ROS production while high concentrations regulated excessive ROS production, possibly by blocking assembly of $\mathrm{p} 47^{\mathrm{phox}}$ on NADPH oxidase. Blocking of $\mathrm{p} 47^{\text {phox }}$ assembly is also the mechanism by which porcine PR-39 is able to regulate activated neutrophil ROS production. However, PR-39 achieves this by binding to $\mathrm{p} 47^{\mathrm{phox}}$, rather than $\beta$-actin. The existence of two structurally similar cathelicidin AMPs which achieve a similar regulatory effect using slightly different mechanisms highlights the importance of the regulation of ROS production during neutrophil inflammatory responses. The most likely reason for this regulatory activity may be to prevent oxidative stress and damage to host cells. However, Bac5 also enhanced both extracellular and intracellular basal ROS production, suggesting that some of the activity exhibited by Bac5, in addition to $\beta$-actin interactions, may also involve ROS signalling. Further research is required to establish if this is the case.

Taken together, the results of this thesis describe multifaceted roles for the bovine cathelicidins in host defence, which may coordinate to enhance function but also regulate excessive pro-inflammatory responses from neutrophils. These potential roles are summarised in the context of infection in vivo as follows (refer to Figure 6.1).

The infiltration of pathogens is recognised by sentinel cells such as dendritic cells and macrophages (a). These cells respond to pathogen exposure by releasing chemokines to recruit neutrophils from the circulation (b). Epithelial cells also release chemokines upon recognition of pathogens (c). Neutrophils migrate from blood vessels, through the basement membrane, and between epithelial cells toward the area of infection using the chemoattractant gradients established by the epithelial and sentinel cells. (d). Here, the neutrophils may encounter pathogens directly (e), triggering degranulation of humoral components, or, chemokines secreted by the sentinel cells in response to pathogen (e) may also induce neutrophil degranulation. At this point the cathelicidins are released from the large granules and can be processed into the CLD and AMP domain by primary granule constituents. As the infiltration of neutrophils is rapid and pronounced, so too is the release of cathelicidins into the inflammatory environment. 


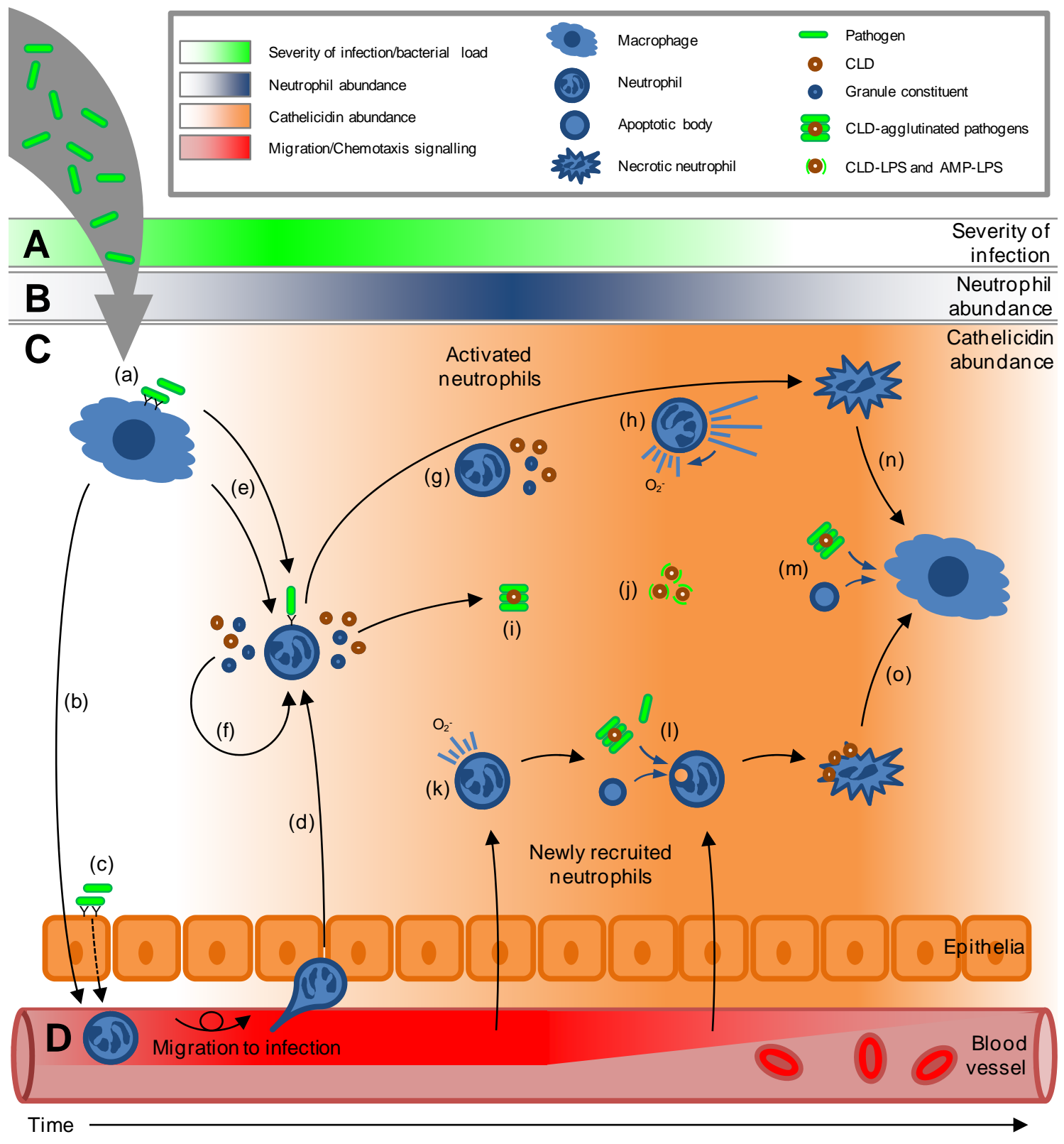

\section{Figure 6.1 Summary of the roles of bovine cathelicidins in host defence}

For $(\mathbf{A})-(\mathbf{D})$, shading indicates the abundance relative to time. As time proceeds during infection, the severity of the bacterial load rapidly increases (A). As pathogens are detected, chemoattractant signalling surges (D) resulting in a rapid and pronounced infiltration of neutrophils to the site of infection (B). As neutrophils accumulate, the concentration of cathelicidins, both the CLD and AMPs, increases through neutrophil degranulation $(\mathbf{C})$. Steps (a) - (o) are described in the text. 
As different bovine cathelicidin AMPs are capable of enhancing migration at different concentrations, a minor positive feedback loop may be created (f) which further promotes neutrophil interaction with pathogens or cytokines. Neutrophils activated in this manner are further induced to degranulate by the increasing concentration of certain AMPs (g). Activated neutrophils are also likely to begin producing large amounts of ROS, however ROS production may be held in check by high concentrations of Bac5 (h) or other peptides, thereby preventing oxidative damage of other immune cells and surrounding tissues.

The parallel increase in CLD would begin to cause pathogen agglutination that would help to prevent the spread of infection (i). With continued degranulation and release of cathelicidins, the concentration of CLD and AMPs may quickly have a significant effect on the amount of free LPS (j) in the environment. The sequestration of LPS by both the CLD and AMPs may lead to a reduction in LPS-stimulated chemoattractant release by epithelial and sentinel cells, slowing the rate of migration of neutrophils into the inflammatory focus.

Newly recruited neutrophils may not directly encounter pathogen. Despite this, different AMPs may act to enhance basal ROS production (k) to assist in pathogen elimination. The high concentration of AMPs may also act on newly recruited neutrophils to enhance phagocytosis, and in a coordinated effort with the CLD, aid in the clearance of agglutinated pathogens (1). Apoptotic or spent neutrophils may also be cleared via this enhanced phagocytic response. Macrophages would assist in these processes too (m). However as the infection is brought under control, an excess of neutrophils can lead to unnecessary damage to host tissues. While less pathogen recognition may have led to a drop in neutrophil infiltration rate, high concentrations of certain AMPs would induce neutrophil necrosis, allowing excess neutrophils to be cleared from the inflammatory focus (n). High concentrations of CLD would similarly induce necrosis in a concerted effort to promote clearance by macrophages (o).

It is important to keep in mind that the effects of the cathelicidins were minor in some instances, and that other endogenous or exogenous signals may dominate over the effects induced by the cathelicidins. Although it is still unclear as to the range of 
concentrations at which bovine cathelicidins are bioavailable during infection, in most species the abundance of cathelicidins during infection is high. Therefore their impact on innate immune responses is likely to be significant.

In summary, the bovine cathelicidins have multiple roles in host defence. The conserved CLD appears to have an anti-inflammatory role through an as yet undefined mechanism. The variable AMPs together have multifaceted roles which may act in concert to promote elimination of pathogens and regulate excessive detrimental neutrophil activity. The combined effect of these roles may act to enhance clearance of pathogens during infection and aid in the resolution of the innate inflammatory response. 


\subsection{Opportunities for future research}

\subsubsection{The origin of the immunomodulatory activity of the CLD}

The work in this thesis clarifies a role for the bovine CLD in humoral and cellular innate immunity. However, the mechanism by which the CLD promotes microbe agglutination and neutrophil death was not identified. The reason for the activity exhibited by the native Cath1 preparation was likely due to the presence of a higher molecular weight form of the CLD. This has previously been observed in bovine but has not been completely characterised [58]. Hence, there are several questions about the activity of the CLD that need to be addressed.

First and foremost, is the higher molecular weight protein the cause of the observed immunomodulatory activity? The best way to address this question would be to separate the different molecular weights by size exclusion chromatography and characterise the function of each CLD fraction separately. Hence, if the high molecular weight complex is the cause of the activity, why is it only present in the native Cath 1 sample? As the protein would be lethal from the onset of translation, a structural rearrangement probably occurs after neutrophil degranulation during infection. This might also explain why the recombinant proteins could only be produced as monomers or dimers in bacteria. Exactly what conditions might cause this higher oligomerisation are unclear. However, the domain swapped dimers formed by protegrin-3 [97,109] were produced under acidic crystallisation conditions. Therefore lowering the $\mathrm{pH}$ of solutions containing recombinant CLD protein may allow multimeric structures to spontaneously form. Additionally, lowering the $\mathrm{pH}$ of the solutions used for on-column refolding may also give the desired result.

Are the multimeric structures regular and ordered, or aggregates of misfolded protein? The increments in molecular weight observed in the western blot correspond to multimers containing an even number of monomers. Also, proline-rich regions in many proteins can act as 'quaternary structure helpers' [274] aiding oligomerisation. The loop section between $\beta$-sheets one and two of the CLD (the anti-parallel link in the dimerised structure [97]) contains a high content of proline and could function in this manner. These details suggest that the structures are regular and ordered. To address this 
question, the different molecular weight fractions of the CLD could be subjected to crystallisation trials and analysed by X-ray crystallography or by solution NMR spectroscopy to determine if there is order to the structures.

Considering the CLD is highly conserved across species, could this be a general property of all CLDs? To address this question the additional six bovine CLDs and CLDs from other species could be purified following the same protocol as native Cath1. Western blot analysis, size exclusion chromatography and functional and structural characterisation would determine whether the oligomeric structure of the CLD and any accompanying function are unique to bovine cathelicidin-1, unique to bovine, or common across species.

\subsubsection{Toward identifying in vivo production of the RITK peptide}

The potential secondary cleavage of cathelicidin-1 was of significant interest as this process would generate a novel undescribed bovine cathelicidin AMP. Although the RITK peptide could not be found after ex vivo stimulation of bovine neutrophils with either PMA or calcium ionophore, several other experiments may provide evidence for production of the peptide in vivo. Firstly, in vitro production of the peptide using rbCath1 and purified endogenous bovine elastase (or other bovine serine proteases) needs to be assessed. This would determine if the peptide can be produced by bovine neutrophil proteases. Secondly, if the peptide is produced, then the use of a more physiological activator for ex vivo stimulation, such as live bacteria, may provide the necessary conditions for production of the peptide. Finally, only extracellular release of the peptide was tested. The peptide may be produced in phagosomes or lysosomes, and MS analysis of neutrophil lysate after stimulation may reveal the peptide.

\subsubsection{Establishing the mechanisms of linear Bac1 and BMAP-34 activity}

Linear Bac1 and BMAP-34 exhibited dose-dependent effects on neutrophil function in the absence of intracellular calcium mobilisation. For linear Bac1 this included enhanced granule release, migration, ROS production, and phagocytosis. Linear Bac1 may be able to cross the plasma membrane and modulate neutrophil function by an intracellular mechanism. At high concentrations, BMAP-34 was able to enhance 
phagocytosis but was also cytotoxic. BMAP-34 also enhanced granule release and migration, but inhibited ROS production in naïve cells. The interaction of BMAP-34 with the neutrophil membrane is unclear. Therefore, similar experiments to those employed to determine the mechanism of Bac5 function could be used here.

\subsubsection{Establishing the follow-on mechanisms of Bac5-actin interactions}

The mechanism of Bac5 regulation of ROS production was not completely clarified. Does Bac5 actually prevent the binding of $\mathrm{p} 47^{\text {phox }}$ to the plasma membrane NADPH oxidase assembly? To address this, a dot blot-binding assay could be used in which purified $\beta$-actin is immobilised on a transfer membrane, blocked, and probed with Bac5 to create Bac5- $\beta$-actin complexes. This could then be probed with purified $\mathrm{p} 47^{\text {phox }}$ to determine if Bac5 blocks the binding of $\mathrm{p} 47^{\text {phox }}$ to actin. This technique assesses binding of proteins in their native state and should accurately reflect what occurs within neutrophils.

The interaction of Bac5 with actin may also play a role in the Bac5-induced modulation of migration, degranulation and phagocytosis. These neutrophil functions are heavily reliant on cytoskeletal reorganisation [258,275] suggesting the Bac5-actin interaction may have further follow-on effects on the cytoskeleton. Firstly, does Bac5 directly affect actin polymerisation or depolymerisation? The simplest method to address this question would be to use a standard actin polymerisation assay kit to measure differences in actin polymerisation in the presence and absence of Bac5. The addition of actin destabilising reagents would also provide data on whether Bac5 stabilises actin filaments that are already established.

Secondly, does the Bac5-actin complex bind other actin proteins? Together there are at least 100 actin-binding proteins from 30 different families [276]. Many of these proteins contain poly-proline binding motifs [249] which are possible targets for Bac5 interactions. A Bac5-actin interaction with other actin-binding proteins warrants future investigation. A possible approach to identifying actin-binding proteins that interact with Bac5-actin might involve an in-depth co-immunopurification analysis using Bac5treated neutrophil lysates. 
Additionally, a series of actin inhibitors (e.g. cytochalasins, phalloidin, latrunculin) could be used to establish which cytoskeletal remodelling processes are essential for Bac5-induced modulation of neutrophil function. This would clarify whether Bac5 indirectly affects actin polymerisation, depolymerisation or both. This would also narrow down the number of possible interacting target proteins. 


\section{References}

1. Chacon, O., Bermudez, L. E., \& Barletta, R. G. (2004). Johne's disease, inflammatory bowel disease, and Mycobacterium paratuberculosis. Annual Review of Microbiology, 58, 329-363.

2. Wu, C.-w., Livesey, M., Schmoller, S. K., Manning, E. J. B., Steinberg, H., Davis, W. C., Hamilton, M. J., \& Talaat, A. M. (2007). Invasion and persistence of Mycobacterium avium subsp. paratuberculosis during early stages of Johne's disease in calves. Infection and Immunity, 75, 2110-2119.

3. Linnabary, R. D., Meerdink, G., Collins, M., Stabel, J., Sweeney, R., Washington, M., Wells, S., Chiodini, R., Hansen, D., \& Whitlock, R. (2001). Johne's disease in cattle: Council for Agricultural Science and Technology.

4. Ott, S. L., Wells, S. J., \& Wagner, B. A. (1999). Herd-level economic losses associated with Johne's disease on US dairy operations. Preventive Veterinary Medicine, 40, 179-192.

5. Heuer, C., Wilson, P., \& Larking, K. (2011). Johne's disease in New Zealand livestock. Vetscript, 24, 39-41.

6. Srikumaran, S., Kelling, C. L., \& Ambagala, A. (2007). Immune evasion by pathogens of bovine respiratory disease complex. Animal Health Research Reviews, 8, 215.

7. Taylor, J. D., Fulton, R. W., Lehenbauer, T. W., Step, D. L., \& Confer, A. W. (2010). The epidemiology of bovine respiratory disease: What is the evidence for predisposing factors? The Canadian Veterinary Journal, 51, 1095.

8. Snowder, G., Van Vleck, L. D., Cundiff, L., \& Bennett, G. (2006). Bovine respiratory disease in feedlot cattle: environmental, genetic, and economic factors. Journal of Animal Science, 84, 1999-2008.

9. Malcolm, D., Hook, I., Montgomerie, B., Winkleman, A., Sim, S., Brennan, G., Lacy-Hulbert, J., Franks, B., Hawkins, D., Reidy, P., Andela, R., Williams, D., \& McDougall, S. (2006). The cost of mastitis. Dairy Insight Research 2005/2006 report of the National Mastitis Advisory Committee of New Zealand.

10. McDougall, S., Arthur, D. G., Bryan, M. A., Vermunt, J. J., \& Weir, A. M. (2007). Clinical and bacteriological response to treatment of clinical mastitis 
with one of three intramammary antibiotics. New Zealand Veterinary Journal, 55, 161-170.

11. Tiwari, J., Babra, C., Tiwari, H., Williams, V., \& Wet, S. (2013). Trends in therapeutic and prevention strategies for management of bovine mastitis: An overview. Journal of Vaccines \& Vaccination, 4, 2.

12. Stelwagen, K., Carpenter, E., Haigh, B., Hodgkinson, A., \& Wheeler, T. T. (2009). Immune components of bovine colostrum and milk. Journal of Animal Science, 87, 3-9.

13. Wheeler, T. T., Hodgkinson, A. J., Prosser, C. G., \& Davis, S. R. (2007). Immune components of colostrum and milk-a historical perspective. Journal of Mammary Gland Biology and Neoplasia, 12, 237-247.

14. Rainard, P., \& Riollet, C. (2006). Innate immunity of the bovine mammary gland. Veterinary Research, 37, 369-400.

15. Sordillo, L. M., \& Streicher, K. L. (2002). Mammary gland immunity and mastitis susceptibility. Journal of Mammary Gland Biology and Neoplasia, 7, 135-146.

16. Artis, D. (2008). Epithelial-cell recognition of commensal bacteria and maintenance of immune homeostasis in the gut. Nature Reviews Immunology, 8, 411-420.

17. Capuco, A. V., Bright, S. A., Pankey, J. W., Wood, D. L., Miller, R. H., \& Bitman, J. (1992). Increased susceptibility to intramammary infection following removal of teat canal keratin. Journal of Dairy Science, 75, 2126-2130.

18. Viguier, C., Arora, S., Gilmartin, N., Welbeck, K., \& O'Kennedy, R. (2009). Mastitis detection: Current trends and future perspectives. Trends in Biotechnology, 27, 486-493.

19. Seykora, A. J., \& McDaniel, B. T. (1985). Udder and teat morphology related to mastitis resistance: A review. Journal of Dairy Science, 68, 2087-2093.

20. Nickerson, S. C., \& Pankey, J. W. (1983). Cytologic observations of the bovine teat end. American Journal of Veterinary Research, 44, 1433-1441.

21. Thayer, T. (2007). Udders and mastitis. El Lechero (The Dairyman), (3). Retrieved from http://www.progressivedairy.com

22. Takeda, K., \& Akira, S. (2005). Toll-like receptors in innate immunity. International Immunology, 17, 1-14. 
23. Brown, G. D., Gow, N. A. R., Kullberg, B. J., \& Netea, M. G. (2008). An integrated model of the recognition of Candida albicans by the innate immune system. [Clinical report]. Nature Reviews Microbiology, 6, 67+.

24. Gordon, S. (2002). Pattern recognition receptors: Doubling up for the innate immune response. Cell, 111, 927-930.

25. Brown, G. D. (2006). Dectin-1: A signalling non-TLR pattern-recognition receptor. Nature Reviews Immunology, 6, 33-43.

26. Raghavan, M., \& Bjorkman, P. J. (1996). Fc receptors and their interactions with immunoglobulins. Annual Review of Cell and Developmental Biology, 12, 181220.

27. Saad, A. M., \& Ostensson, K. (1990). Flow cytofluorometric studies on the alteration of leukocyte populations in blood and milk during endotoxin-induced mastitis in cows. American Journal of Veterinary Research, 51, 1603-1607.

28. Beutler, B. (2004). Innate immunity: An overview. Molecular Immunology, 40, 845-859.

29. Tedder, T. F., Steeber, D. A., Chen, A., \& Engel, P. (1995). The selectins: Vascular adhesion molecules. The FASEB Journal, 9, 866-873.

30. Quesnell, R. R., Erickson, J., \& Schultz, B. D. (2007). Apical electrolyte concentration modulates barrier function and tight junction protein localization in bovine mammary epithelium. American Journal of Physiology - Cell Physiology, 292, C305-C318.

31. Heit, B., Tavener, S., Raharjo, E., \& Kubes, P. (2002). An intracellular signaling hierarchy determines direction of migration in opposing chemotactic gradients. The Journal of Cell Biology, 159, 91-102.

32. Selvatici, R., Falzarano, S., Mollica, A., \& Spisani, S. (2006). Signal transduction pathways triggered by selective formylpeptide analogues in human neutrophils. European Journal of Pharmacology, 534, 1-11.

33. Fittschen, C., Sandhaus, R., Worthen, G., \& Henson, P. (1988). Bacterial lipopolysaccharide enhances chemoattractant-induced elastase secretion by human neutrophils. Journal of Leukocyte Biology, 43, 547-556.

34. Gray, G., Knight, K., Nelson, R., \& Herron, M. (1982). Chemotactic requirements of bovine leukocytes. American Journal of Veterinary Research, 43, 757. 
35. Carroll, E., Mueller, R., \& Panico, L. (1982). Chemotactic factors for bovine leukocytes. American Journal of Veterinary Research, 43, 1661.

36. Gerard, C., \& Hugli, T. E. (1981). Identification of classical anaphylatoxin as the des-Arg form of the C5a molecule: Evidence of a modulator role for the oligosaccharide unit in human des-Arg74-C5a. Proceedings of the National Academy of Sciences, 78, 1833-1837.

37. Gennaro, R., Simonic, T., Negri, A., Mottola, C., Secchi, C., Ronchi, S., \& Romeo, D. (1986). C5a fragment of bovine complement. European Journal of Biochemistry, 155, 77-86.

38. Bannerman, D. D. (2009). Pathogen-dependent induction of cytokines and other soluble inflammatory mediators during intramammary infection of dairy cows. Journal of Animal Science, 87, 10-25.

39. Pighetti, G. M., \& Rambeaud, M. (2006). Genome conservation between the bovine and human interleukin-8 receptor complex: Improper annotation of bovine interleukin-8 receptor $\mathrm{b}$ identified. Veterinary Immunology and Immunopathology, 114, 335-340.

40. Galligan, C. L., \& Coomber, B. L. (2000). Effects of human IL-8 isoforms on bovine neutrophil function in vitro. Veterinary Immunology and Immunopathology, 74, 71-85.

41. Pighetti, G. M., Kojima, C. J., Wojakiewicz, L., \& Rambeaud, M. (2012). The bovine CXCR1 gene is highly polymorphic. Veterinary Immunology and Immunopathology, 145, 464-470.

42. Soehnlein, O., Weber, C., \& Lindbom, L. (2009). Neutrophil granule proteins tune monocytic cell function. Trends in Immunology, 30, 538-546.

43. Jog, N. R., Rane, M. J., Lominadze, G., Luerman, G. C., Ward, R. A., \& McLeish, K. R. (2007). The actin cytoskeleton regulates exocytosis of all neutrophil granule subsets. American Journal of Physiology - Cell Physiology, 292, C1690-C1700.

44. Bainton, D. F., Ullyot, J. L., \& Farquhar, M. G. (1971). The development of neutrophilic polymorphonuclear leukocytes in human bone marrow: Origin and content of azurophil and specific granules. The Journal of Experimental Medicine, 134, 907-934. 
45. Gennaro, R., Dewald, B., \& Horisberger, U. (1983). A novel type of cytoplasmic granule in bovine neutrophils. Journal of Cell Biology, 96, 1651-1661.

46. Sørensen, O., Arnljots, K., Cowland, J. B., Bainton, D. F., \& Borregaard, N. (1997). The human antibacterial cathelicidin, hCAP-18, is synthesized in myelocytes and metamyelocytes and localized to specific granules in neutrophils. Blood, 90, 2796-2803.

47. Borregaard, N., \& Cowland, J. B. (1997). Granules of the human neutrophilic polymorphonuclear leukocyte. Blood, 89, 3503-3521.

48. Hampton, M. B., Kettle, A. J., \& Winterbourn, C. C. (1998). Inside the neutrophil phagosome: Oxidants, myeloperoxidase, and bacterial killing. Blood, 92, 3007-3017.

49. Holland, S. M. (2010). Chronic granulomatous disease. Clinical Reviews in Allergy \& Immunology, 38, 3-10.

50. Wilkinson, B. L., \& Landreth, G. E. (2006). The microglial NADPH oxidase complex as a source of oxidative stress in Alzheimer's disease. Journal of Neuroinflammation, 3, 30.

51. Paape, M. J., Bannerman, D. D., Zhao, X., \& Lee, J. W. (2003). The bovine neutrophil: Structure and function in blood and milk. Veterinary Research, 34, 597-627.

52. Ravetch, J. V., \& Clynes, R. A. (1998). Divergent roles for Fc receptros and complement in vivo. Annual Review of Immunology, 16, 421-432.

53. Bazzoni, F., Cassatella, M. A., Rossi, F., Ceska, M., Dewald, B., \& Baggiolini, M. (1991). Phagocytosing neutrophils produce and release high amounts of the neutrophil-activating peptide 1/interleukin 8. Journal of Experimental Medicine, 173, 771-774.

54. Murray, R. Z., Kay, J. G., Sangermani, D. G., \& Stow, J. L. (2005). Cell biology: A role for the phagosome in cytokine secretion. Science, 310, 14921495.

55. Serhan, C. N., \& Savill, J. (2005). Resolution of inflammation: The beginning programs the end. Nature Immunology, 6, 1191-1197.

56. Smits, E., Burvenich, C., Guidry, A. J., Heyneman, R., \& Massart-Leën, A. (1999). Diapedesis across mammary epithelium reduces phagocytic and 
oxidative burst of bovine neutrophils. Veterinary Immunology and Immunopathology, 68, 169-176.

57. Paape, M., \& Guidry, A. (1977). Effect of fat and casein on intracellular killing of Staphylococcus aureus by milk leukocytes. Paper presented at the Proceedings of the Society for Experimental Biology and Medicine. Society for Experimental Biology and Medicine (New York, NY).

58. Smolenski, G., Haines, S., Kwan, F. Y. S., Bond, J., Farr, V., Davis, S. R., Stelwagen, K., \& Wheeler, T. T. (2007). Characterisation of host defence proteins in milk using a proteomic approach. Journal of Proteome Research, 6, 207-215.

59. Bowdish, D. M. E., \& Hancock, R. E. W. (2005). Anti-endotoxin properties of cationic host defence peptides and proteins. Journal of Endotoxin Research, 11, 230-236.

60. Ricklin, D., Hajishengallis, G., Yang, K., \& Lambris, J. D. (2010). Complement: A key system for immune surveillance and homeostasis. Nature Immunology, 11, 785-797.

61. Rainard, P., Sarradin, P., Paape, M., \& Poutrel, B. (1998). Quantification of C5a/C5a (desArg) in bovine plasma, serum and milk. Veterinary Research, 29, 73-88.

62. Nemali, S., Siemsen, D. W., Nelson, L. K., Bunger, P. L., Faulkner, C. L., Rainard, P., Gauss, K. A., Jutila, M. A., \& Quinn, M. T. (2008). Molecular analysis of the bovine anaphylatoxin $\mathrm{C} 5 \mathrm{a}$ receptor. Journal of Leukocyte Biology, 84, 537-549.

63. Webster, R. O., Hong, S. R., Johnston Jr, R. B., \& Henson, P. M. (1980). Biological effects of the human complement fragments C5a and $\mathrm{C}^{5} \mathrm{a}_{\mathrm{des}} \mathrm{Arg}$ on neutrophil function. Immunopharmacology, 2, 201-219.

64. Kagnoff, M. F., \& Eckmann, L. (1997). Epithelial cells as sensors for microbial infection. Journal of Clinical Investigation, 100, 6-10.

65. Bals, R., \& Hiemstra, P. (2004). Innate immunity in the lung: how epithelial cells fight against respiratory pathogens. European Respiratory Journal, 23, 327-333. 
66. Jäger, S., Stange, E. F., \& Wehkamp, J. (2010). Antimicrobial peptides in gastrointestinal inflammation. International journal of inflammation, vol. 2010, 11 pages. Retrieved from doi:10.4061/2010/910283

67. Tomasinsig, L., De Conti, G., Skerlavaj, B., Piccinini, R., Mazzilli, M., D'Este, F., Tossi, A., \& Zanetti, M. (2010). Broad-spectrum activity against bacterial mastitis pathogens and activation of mammary epithelial cells support a protective role of neutrophil cathelicidins in bovine mastitis. Infection and Immunity, 78, 1781-1788.

68. Tomasinsig, L., Scocchi, M., Di Loreto, C., Artico, D., \& Zanetti, M. (2002). Inducible expression of an antimicrobial peptide of the innate immunity in polymorphonuclear leukocytes. Journal of Leukocyte Biology, 72, 1003-1010.

69. Zanetti, M. (2005). The role of cathelicidins in the innate host defenses of mammals. Current Issues in Molecular Biology, 7, 179-196.

70. Shennan, D. B., \& Peaker, M. (2000). Transport of milk constituents by the mammary gland. Physiological Reviews, 80, 925-951.

71. Mather, I. H. (2000). A review and proposed nomenclature for major proteins of the milk-fat globule membrane. Journal of Dairy Science, 83, 203-247.

72. Goldammer, T., Zerbe, H., Molenaar, A., Schuberth, H. J., Brunner, R. M., Kata, S. R., \& Seyfert, H. M. (2004). Mastitis increases mammary mRNA abundance of $\beta$-defensin 5, Toll-like-receptor 2 (TLR2), and TLR4 but not TLR9 in cattle. Clinical and Diagnostic Laboratory Immunology, 11, 174-185.

73. Clare, D. A., \& Swaisgood, H. E. (2000). Bioactive milk peptides: A prospectus. Journal of Dairy Science, 83, 1187-1195.

74. Gill, H. S., Doull, F., Rutherfurd, K. J., \& Cross, M. L. (2000). Immunoregulatory peptides in bovine milk. British Journal of Nutrition, 84, 111-117.

75. Paape, M. J., Lilius, E. M., Wiitanen, P. A., Kontio, M. P., \& Miller, R. H. (1996). Intramammary defense against infections induced by Escherichia coli in cows. American Journal of Veterinary Research, 57, 477-482.

76. Haziot, A., Chen, S., Ferrero, E., Low, M. G., Silber, R., \& Goyert, S. M. (1988). The monocyte differentiation antigen, CD14, is anchored to the cell membrane by a phosphatidylinositol linkage. The Journal of Immunology, 141, $547-552$. 
77. Prokopowicz, Z., Marcinkiewicz, J., Katz, D. R., \& Chain, B. M. (2012). Neutrophil myeloperoxidase: Soldier and statesman. Archivum Immunologiae et Therapiae Experimentalis, 60, 43-54.

78. Farnaud, S., \& Evans, R. W. (2003). Lactoferrin-a multifunctional protein with antimicrobial properties. Molecular Immunology, 40, 395-405.

79. Elsbach, P., \& Weiss, J. (1998). Role of the bactericidal/permeability-increasing protein in host defence. Current Opinion in Immunology, 10, 45-49.

80. Leong, S. R., \& Camerato, T. (1990). Nucleotide sequence of the bovine bactericidal permeability increasing protein (BPI). Nucleic Acids Research, 18, 3052 .

81. Rausch, P., \& Moore, T. (1975). Granule enzymes of polymorphonuclear neutrophils: A phylogenetic comparison. Blood, 46, 913-919.

82. Ganz, T. (2003). Defensins: Antimicrobial peptides of innate immunity. Nature Reviews Immunology, 3, 710-720.

83. Selsted, M. E., \& Ouellette, A. J. (2005). Mammalian defensins in the antimicrobial immune response. Nature Immunology, 6, 551-557.

84. Savoini, A., Marzari, R., \& Dolzani, L. (1984). Wide-spectrum antibiotic activity of bovine granulocyte polypeptides. Antimicrobial Agents and Chemotherapy, 26, 405-407.

85. Gennaro, R., Skerlavaj, B., \& Romeo, D. (1989). Purification, composition, and activity of two bactenecins, antibacterial peptides of bovine neutrophils. Infection and Immunity, 57, 3142-3146.

86. Romeo, D., Skerlavaj, B., Bolognesi, M., \& Gennaro, R. (1988). Structure and bactericidal activity of an antibiotic dodecapeptide purified from bovine neutrophils. Journal of Biological Chemistry, 263, 9573-9575.

87. Storici, P., Del Sal, G., Schneider, C., \& Zanetti, M. (1992). cDNA sequence analysis of an antibiotic dodecapeptide from neutrophils. FEBS Letters, 314, 187-190.

88. Clamp, M., Cuff, J., Searle, S. M., \& Barton, G. J. (2004). The jalview java alignment editor. Bioinformatics, 20, 426-427.

89. Scocchi, M., Wang, S., \& Zanetti, M. (1997). Structural organization of the bovine catheclidin gene family and identification of a novel number. FEBS Letters, 417, 311-315. 
90. Zhu, S. (2008). Positive selection targeting the cathelin-like domain of the antimicrobial cathelicidin family. Cellular and Molecular Life Sciences, 65, 1285-1294.

91. Apweiler, R., O'onovan, C., Magrane, M., Alam-Faruque, Y., Antunes, R., Bely, B., Bingley, M., Bower, L., Bursteinas, B., \& Chavali, G. (2012). Reorganizing the protein space at the Universal Protein Resource (UniProt). Nucleic Acids Research, 40, D71-D75.

92. Gillenwaters, E. N., Seabury, C. M., Elliott, J. S., \& Womack, J. E. (2009). Sequence analysis and polymorphism discovery in 4 members of the bovine cathelicidin gene family. Journal of Heredity, 100, 241-245.

93. Boehmer, J. L., Degrasse, J. A., Lancaster, V. A., McFarland, M. A., Callahan, J. H., \& Ward, J. L. (2011). Evaluation of protein expression in bovine bronchoalveolar fluid following challenge with Mannheimia haemolytica. Proteomics, 11, 3685-3697.

94. Wang, T.-T., Nestel, F. P., Bourdeau, V., Nagai, Y., Wang, Q., Liao, J., TaveraMendoza, L., Lin, R., Hanrahan, J. H., Mader, S., \& White, J. H. (2004). Cutting edge: 1,25-dihydroxyvitamin D3 is a direct inducer of antimicrobial peptide gene expression. The Journal of Immunology, 173, 2909-2912.

95. Gombart, A. F., Borregaard, N., \& Koeffler, H. P. (2005). Human cathelicidin antimicrobial peptide (CAMP) gene is a direct target of the vitamin D receptor and is strongly up-regulated in myeloid cells by 1, 25-dihydroxyvitamin $\mathrm{D} 3$. The FASEB journal, 19, 1067-1077.

96. Nelson, C. D., Reinhardt, T. A., Lippolis, J. D., Sacco, R. E., \& Nonnecke, B. J. (2012). Vitamin D signaling in the bovine immune system: A model for understanding human vitamin D requirements. Nutrients, 4, 181-196.

97. Sanchez, J. F., Hoh, F., Strub, M. P., Aumelas, A., \& Dumas, C. (2002). Structure of the cathelicidin motif of protegrin-3 precursor: Structural insights into the activation mechanism of an antimicrobial protein. Structure, 10, 13631370.

98. Yang, Y., Sanchez, J. F., Strub, M. P., Brutscher, B., \& Aumelas, A. (2003). NMR structure of the cathelin-like domain of the protegrin-3 precursor. Biochemistry, 42, 4669-4680. 
99. Pazgier, M., Ericksen, B., Ling, M., Toth, E., Shi, J., Li, X., Galliher-Beckley, A., Lan, L., Zou, G., Zhan, C., Yuan, W., Pozharski, E., \& Lu, W. (2013). Structural and Functional Analysis of the Pro-Domain of Human Cathelicidin, LL-37. Biochemistry, 52, 1547-1558.

100. Kabsch, W., \& Sander, C. (1983). Dictionary of protein secondary structure: pattern recognition of hydrogen-bonded and geometrical features. Biopolymers Peptide Science Section, 22, 2577-2637.

101. Zaiou, M., \& Gallo, R. L. (2002). Cathelicidins, essential gene-encoded mammalian antibiotics. Journal of Molecular Medicine, 80, 549-561.

102. Zanetti, M. (2004). Cathelicidins, multifunctional peptides of the innate immunity. Journal of Leukocyte Biology, 75, 39-48.

103. Shinnar, A. E., Butler, K. L., \& Park, H. J. (2003). Cathelicidin family of antimicrobial peptides: Proteolytic processing and protease resistance. Bioorganic Chemistry, 31, 425-436.

104. Cole, A. M., Shi, J., Ceccarelli, A., Kim, Y. H., Park, A., \& Ganz, T. (2001). Inhibition of neutrophil elastase prevents cathelicidin activation and impairs clearance of bacteria from wounds. Blood, 97, 297-304.

105. Scocchi, M., Skerlavaj, B., Romeo, D., \& Gennaro, R. (1992). Proteolytic cleavage by neutrophil elastase converts inactive storage proforms to antibacterial bactenecins. European Journal of Biochemistry, 209, 589-595.

106. Sørensen, O. E., Follin, P., Johnsen, A. H., Calafat, J., Sandra Tjabringa, G., Hiemstra, P. S., \& Borregaard, N. (2001). Human cathelicidin, hCAP-18, is processed to the antimicrobial peptide LL-37 by extracellular cleavage with proteinase 3. Blood, 97, 3951-3959.

107. Chromek, M., Slamová, Z., Bergman, P., Kovács, L., Podracká, L. u., Ehrén, I., Hökfelt, T., Gudmundsson, G. H., Gallo, R. L., \& Agerberth, B. (2006). The antimicrobial peptide cathelicidin protects the urinary tract against invasive bacterial infection. Nature Medicine, 12, 636-641.

108. Smolenski, G. A., Wieliczko, R. J., Pryor, S. M., Broadhurst, M. K., Wheeler, T. T., \& Haigh, B. J. (2011). The abundance of milk cathelicidin proteins during bovine mastitis. Veterinary Immunology and Immunopathology, 143, 125-130.

109. Sanchez, J. F., Wojcik, F., Yang, Y. S., Strub, M. P., Strub, J. M., Van Dorsselaer, A., Martin, M., Lehrer, R., Ganz, T., Chavanieu, A., Calas, B., \& 
Aumelas, A. (2002). Overexpression and structural study of the cathelicidin motif of the protegrin-3 precursor. Biochemistry, 41, 21-30.

110. Sørensen, O., Cowland, J. B., Askaa, T., \& Borregaard, N. (1997). An ELISA for hCAP-18, the cathelicidin present in human neutrophils and plasma. Journal of Immunological Methods, 206, 53-59.

111. Staniforth, R. A., Giannini, S., Higgins, L. D., Conroy, M. J., Hounslow, A. M., Jerala, R., Craven, C. J., \& Waltho, J. P. (2001). Three-dimensional domain swapping in the folded and molten-globule states of cystatins, an amyloidforming structural superfamily. EMBO Journal, 20, 4774-4781.

112. Janowski, R., Kozak, M., Jankowska, E., Grzonka, Z., Grubb, A., Abrahamson, M., \& Jaskolski, M. (2001). Human cystatin C, an amyloidogenic protein, dimerizes through three-dimensional domain swapping. Nature Structural Biology, 8, 316-320.

113. Kopitar, M., Ritonja, A., Popovic, T., Gabrijelcic, D., Krizaj, I., \& Turk, V. (1989). A new type of low-molecular mass cysteine proteinase inhibitor from pig leukocytes. Biological Chemistry Hoppe-Seyler, 370, 1145-1151.

114. Lenarčič, B., Ritonja, A., Dolenc, I., Stoka, V., Berbič, S., Pungerčar, J. e., Štrukelj, B., \& Turk, V. (1993). Pig leukocyte cysteine proteinase inhibitor (PLCPI), a new member of the stefin family. FEBS Letters, 336, 289-292.

115. Zaiou, M., Nizet, V., \& Gallo, R. L. (2003). Antimicrobial and protease inhibitory functions of the human cathelicidin (hCAP18/LL-37) prosequence. Journal of Investigative Dermatology, 120, 810-816.

116. Zhu, S., Wei, L., Yamasaki, K., \& Gallo, R. L. (2008). Activation of cathepsin L by the cathelin-like domain of protegrin-3. Molecular Immunology, 45, 25312536.

117. Verbanac, D., Zanetti, M., \& Romeo, D. (1993). Chemotactic and proteaseinhibiting activities of antibiotic peptide precursors. FEBS Letters, 317, 255-258.

118. Guaní-Guerra, E., Santos-Mendoza, T., Lugo-Reyes, S. O., \& Terán, L. M. (2010). Antimicrobial peptides: General overview and clinical implications in human health and disease. Clinical Immunology, 135, 1-11.

119. Xiao, Y., Cai, Y., Bommineni, Y. R., Fernando, S. C., Prakash, O., Gilliland, S. E., \& Zhang, G. (2006). Identification and functional characterization of three 
chicken cathelicidins with potent antimicrobial activity. Journal of Biological Chemistry, 281, 2858-2867.

120. Méndez-Samperio, P. (2010). The human cathelicidin hCAP18/LL-37: A multifunctional peptide involved in mycobacterial infections. Peptides, 31, 1791-1798.

121. Selsted, M. E., Novotny, M. J., Morris, W. L., Tang, Y. Q., Smith, W., \& Cullor, J. S. (1992). Indolicidin, a novel bactericidal tridecapeptide amide from neutrophils. Journal of Biological Chemistry, 267, 4292-4295.

122. Shamova, O., Brogden, K. A., Zhao, C., Nguyen, T., Kokryakov, V. N., \& Lehrer, R. I. (1999). Purification and properties of proline-rich antimicrobial peptides from sheep and goat leukocytes. Infection and Immunity, 67, 41064111.

123. Rathinakumar, R., \& Wimley, W. C. (2010). High-throughput discovery of broad-spectrum peptide antibiotics. The FASEB journal, 24, 3232-3238.

124. Vaara, M. (2009). New approaches in peptide antibiotics. Current Opinion in Pharmacology, 9, 571-576.

125. de Oca, E. P. M. (2013). Antimicrobial peptide elicitors: New hope for the postantibiotic era. Innate Immunity, 19, 227-241.

126. Leonard, B. C., Chu, H., Johns, J. L., Gallo, R. L., Moore, P. F., Marks, S. L., \& Bevins, C. L. (2011). Expression and activity of a novel cathelicidin from domestic cats. PLoS One, 6, e18756.

127. Skerlavaj, B., Gennaro, R., Bagella, L., Merluzzi, L., Risso, A., \& Zanettit, M. (1996). Biological characterization of two novel cathelicidin-derived peptides and identification of structural requirements for their antimicrobial and cell lytic activities. Journal of Biological Chemistry, 271, 28375-28381.

128. Sang, Y., Teresa Ortega, M., Rune, K., Xiau, W., Zhang, G., Soulages, J. L., Lushington, G. H., Fang, J., Williams, T. D., Blecha, F., \& Melgarejo, T. (2007). Canine cathelicidin (K9CATH): Gene cloning, expression, and biochemical activity of a novel pro-myeloid antimicrobial peptide. Developmental \& Comparative Immunology, 31, 1278-1296.

129. Scocchi, M., Bontempo, D., Boscolo, S., Tomasinsig, L., Giulotto, E., \& Zanetti, M. (1999). Novel cathelicidins in horse leukocytes. FEBS Letters, 457, 459-464. 
130. Agerberth, B., Gunne, H., Odeberg, J., Kogner, P., Boman, H. G., \& Gudmundsson, G. H. (1995). FALL-39, a putative human peptide antibiotic, is cysteine-free and expressed in bone marrow and testis. Proceedings of the National Academy of Sciences of the United States of America, 92, 195-199.

131. Gallo, R. L., Kim, K. J., Bernfield, M., Kozak, C. A., Zanetti, M., Merluzzi, L., \& Gennaro, R. (1997). Identification of CRAMP, a cathelin-related antimicrobial peptide expressed in the embryonic and adult mouse. Journal of Biological Chemistry, 272, 13088-13093.

132. Zanetti, M., Storici, P., Tossi, A., Scocchi, M., \& Gennaro, R. (1994). Molecular cloning and chemical synthesis of a novel antibacterial peptide derived from pig myeloid cells. Journal of Biological Chemistry, 269, 7855-7858.

133. Storici, P., Scocchi, M., Tossi, A., Gennaro, R., \& Zanetti, M. (1994). Chemical synthesis and biological activity of a novel antibacterial peptide deduced from a pig myeloid cDNA. FEBS Letters, 337, 303-307.

134. Tossi, A., Scocchi, M., Zanetti, M., Storici, P., \& Gennaro, R. (1995). PMAP37, a novel antibacterial peptide from pig myeloid cells. cDNA cloning, chemical synthesis and activity. European Journal of Biochemistry, 228, 941946.

135. Chen, C., Brock, R., Luh, F., Chou, P. J., Larrick, J. W., Huang, R. F., \& Huang, T. H. (1995). The solution structure of the active domain of CAP18 - A lipopolysaccharide binding protein from rabbit leukocytes. FEBS Letters, 370, 46-52.

136. Termén, S., Tollin, M., Olsson, B., Svenberg, T., Agerberth, B., \& Gudmundsson, G. H. (2003). Phylogeny, processing and expression of the rat cathelicidin rCRAMP: A model for innate antimicrobial peptides. Cellular and Molecular Life Sciences CMLS, 60, 536-549.

137. Zhao, C., Nguyen, T., Boo, L. M., Hong, T., Espiritu, C., Orlov, D., Wang, W., Waring, A., \& Lehrer, R. I. (2001). RL-37, an alpha-helical antimicrobial peptide of the rhesus monkey. Antimicrobial Agents and Chemotherapy, 45, 2695-2702.

138. Tack, B. F., Sawai, M. V., Kearney, W. R., Robertson, A. D., Sherman, M. A., Wang, W., Hong, T., Boo, L. M., Wu, H., Waring, A. J., \& Lehrer, R. I. (2002). 
SMAP-29 has two LPS-binding sites and a central hinge. European Journal of Biochemistry, 269, 1181-1189.

139. Kokryakov, V. N., Harwig, S. S., Panyutich, E. A., Shevchenko, A. A., Aleshina, G. M., Shamova, O. V., Korneva, H. A., \& Lehrer, R. I. (1993). Protegrins: Leukocyte antimicrobial peptides that combine features of corticostatic defensins and tachyplesins. FEBS Letters, 327, 231-236.

140. Zhao, C., Liu, L., \& Lehrer, R. I. (1994). Identification of a new member of the protegrin family by cDNA cloning. FEBS Letters, 346, 285-288.

141. Bagella, L., Scocchi, M., \& Zanetti, M. (1995). cDNA sequences of three sheep myeloid cathelicidins. FEBS Letters, 376, 225-228.

142. Agerberth, B., Lee, J. Y., Bergman, T., Carlquist, M., Boman, H. G., Mutt, V., \& Jornvall, H. (1991). Amino acid sequence of PR-39. Isolation from pig intestine of a new member of the family of proline-arginine-rich antibacterial peptides. European Journal of Biochemistry, 202, 849-854.

143. Zhao, C., Ganz, T., \& Lehrer, R. I. (1995). Structures of genes for two cathelinassociated antimicrobial peptides: prophenin-2 and PR-39. FEBS Letters, 376, 130-134.

144. Marr, A. K., Gooderham, W. J., \& Hancock, R. E. W. (2006). Antibacterial peptides for therapeutic use: Obstacles and realistic outlook. Current Opinion in Pharmacology, 6, 468-472.

145. Zasloff, M. (1987). Magainins, a class of antimicrobial peptides from Xenopus skin: Isolation, characterization of two active forms, and partial cDNA sequence of a precursor. Proceedings of the National Academy of Sciences of the United States of America, 84, 5449-5453.

146. Lipsky, B. A., Holroyd, K. J., \& Zasloff, M. (2008). Topical versus systemic antimicrobial therapy for treating mildly infected diabetic foot ulcers: A randomized, controlled, double-blinded, multicenter trial of pexiganan cream. Clinical Infectious Diseases, 47, 1537-1545.

147. Rubinchik, E., Dugourd, D., Algara, T., Pasetka, C., \& Friedland, H. D. (2009). Antimicrobial and antifungal activities of a novel cationic antimicrobial peptide, omiganan, in experimental skin colonisation models. International Journal of Antimicrobial Agents, 34, 457-461. 
148. Chennupati, S. K., Chiu, A. G., Tamashiro, E., Banks, C. A., Cohen, M. B., Bleier, B. S., Kofonow, J. M., Tam, E., \& Cohen, N. A. (2009). Effects of an LL-37-derived antimicrobial peptide in an animal model of biofilm Pseudomonas sinusitis. American Journal of Rhinology \& Allergy, 23, 46-51.

149. Yeung, A. Y., Gellatly, S., \& Hancock, R. W. (2011). Multifunctional cationic host defence peptides and their clinical applications. Cellular and Molecular Life Sciences, 68, 2161-2176.

150. De Yang, B., Chen, Q., Schmidt, A. P., Anderson, G. M., Wang, J. M., Wooters, J., Oppenheim, J. J., \& Chertov, O. (2000). LL-37, the neutrophil granule- and epithelial cell-derived cathelicidin, utilizes formyl peptide receptor-like 1 (FPRL1) as a receptor to chemoattract human peripheral blood neutrophils, monocytes, and T cells. Journal of Experimental Medicine, 192, 1069-1074.

151. Elssner, A., Duncan, M., Gavrilin, M., \& Wewers, M. D. (2004). A novel P2X 7 receptor activator, the human cathelicidin-derived peptide LL37, induces IL-1 $\beta$ processing and release. Journal of Immunology, 172, 4987-4994.

152. Zheng, Y., Niyonsaba, F., Ushio, H., Nagaoka, I., Ikeda, S., Okumura, K., \& Ogawa, H. (2007). Cathelicidin LL-37 induces the generation of reactive oxygen species and release of human $\alpha$-defensins from neutrophils. British Journal of Dermatology, 157, 1124-1131.

153. Li, H. N., Barlow, P. G., Bylund, J., Mackellar, A., Björstad, Å., Conlon, J., Hiemstra, P. S., Haslett, C., Gray, M., Simpson, A. J., Rossi, A. G., \& Davidson, D. J. (2009). Secondary necrosis of apoptotic neutrophils induced by the human cathelicidin LL-37 is not proinflammatory to phagocytosing macrophages. Journal of Leukocyte Biology, 86, 891-902.

154. Huang, H. J., Ross, C. R., \& Blecha, F. (1997). Chemoattractant properties of PR-39, a neutrophil antibacterial peptide. Journal of Leukocyte Biology, 61, 624629.

155. Shi, J., Ross, C. R., Leto, T. L., \& Blecha, F. (1996). PR-39, a proline-rich antibacterial peptide that inhibits phagocyte NADPH oxidase activity by binding to Src homology 3 domains of $47^{\text {phox }}$. Proceedings of the National Academy of Sciences of the United States of America, 93, 6014-6018. 
156. Bridle, A., Nosworthy, E., Polinski, M., \& Nowak, B. (2011). Evidence of an antimicrobial-immunomodulatory role of Atlantic salmon cathelicidins during infection with Yersinia ruckeri. PLoS One, 6, e23417.

157. Radermacher, S. W., Schoop, V. M., \& Schluesener, H. J. (1993). Bactenecin, a leukocytic antimicrobial peptide, is cytotoxic to neuronal and glial cells. Journal of Neuroscience Research, 36, 657-662.

158. Risso, A., Braidot, E., Sordano, M. C., Vianello, A., Macrì, F., Skerlavaj, B., Zanetti, M., Gennaro, R., \& Bernardi, P. (2002). BMAP-28, an antibiotic peptide of innate immunity, induces cell death through opening of the mitochondrial permeability transition pore. Molecular and Cellular Biology, 22, 1926-1935.

159. Schluesener, H. J., Radermacher, S., Melms, A., \& Jung, S. (1993). Leukocytic antimicrobial peptides kill autoimmune T cells. Journal of Neuroimmunology, 47, 199-202.

160. Risso, A., Zanetti, M., \& Gennaro, R. (1998). Cytotoxicity and apoptosis mediated by two peptides of innate immunity. Cellular Immunology, 189, $107-$ 115.

161. D'Este, F., Tomasinsig, L., Skerlavaj, B., \& Zanetti, M. (2012). Modulation of cytokine gene expression by cathelicidin BMAP-28 in LPS-stimulated and unstimulated macrophages. Immunobiology, 217, 962-971.

162. Soehnlein, O., Zernecke, A., Eriksson, E. E., Rothfuchs, A. G., Pham, C. T., Herwald, H., Bidzhekov, K., Rottenberg, M. E., Weber, C., \& Lindbom, L. (2008). Neutrophil secretion products pave the way for inflammatory monocytes. Blood, 112, 1461-1471.

163. Wantha, S., Alard, J.-E., Megens, R. T., van der Does, A. M., Döring, Y., Drechsler, M., Pham, C. T. N., Wang, M.-W., Wang, J.-M., Gallo, R., von Hundelshausen, P., Lindbom, L., Hackeng, T. M., Weber, C., \& Soehnlein, O. (2013). Neutrophil-derived cathelicidin promotes adhesion of classical monocytes. Circulation Research, 112, 792-801.

164. Schauber, J., \& Gallo, R. L. (2007). Expanding the roles of antimicrobial peptides in skin: Alarming and arming keratinocytes. Journal of Investigative Dermatology, 127, 510-512. 
165. Scott, M. G., Davidson, D. J., Gold, M. R., Bowdish, D., \& Hancock, R. E. W. (2002). The human antimicrobial peptide LL-37 is a multifunctional modulator of innate immune responses. Journal of Immunology, 169, 3883-3891.

166. Tokumaru, S., Sayama, K., Shirakata, Y., Komatsuzawa, H., Ouhara, K., Hanakawa, Y., Yahata, Y., Dai, X., Tohyama, M., \& Nagai, H. (2005). Induction of keratinocyte migration via transactivation of the epidermal growth factor receptor by the antimicrobial peptide LL-37. The Journal of Immunology, 175, 4662-4668.

167. Chen, X., Takai, T., Xie, Y., Okumura, K., Ikeda, S., \& Ogawa, H. (2013). Modulation of double-stranded RNA-and cytokine-induced responses of human keratinocytes by LL-37. Paper presented at the 37th Annual Meeting of the Japanese Society for Investigative Dermatology, Edinburgh International Conference Centre.

168. Chotjumlong, P., Bolscher, J. G., Nazmi, K., Reutrakul, V., Supanchart, C., Buranaphatthana, W., \& Krisanaprakornkit, S. (2012). Involvement of the P2X purinergic receptor and c-Jun $\mathrm{N}$-terminal and extracellular signal-regulated kinases in cyclooxygenase-2 and prostaglandin E2 induction by LL-37. Journal of Innate Immunity, 5, 72-83.

169. Alalwani, S. M., Sierigk, J., Herr, C., Pinkenburg, O., Gallo, R., Vogelmeier, C., \& Bals, R. (2010). The antimicrobial peptide LL-37 modulates the inflammatory and host defense response of human neutrophils. European Journal of Immunology, 40, 1118-1126.

170. Wan, M., Sabirsh, A., Wetterholm, A., Agerberth, B., \& Haeggström, J. Z. (2007). Leukotriene B4 triggers release of the cathelicidin LL-37 from human neutrophils: Novel lipid-peptide interactions in innate immune responses. The FASEB Journal, 21, 2897-2905.

171. Byfield, F. J., Wen, Q., Leszczyńska, K., Kułakowska, A., Namiot, Z., Janmey, P. A., \& Bucki, R. (2011). Cathelicidin LL-37 peptide regulates endothelial cell stiffness and endothelial barrier permeability. American Journal of Physiology Cell Physiology, 300, C105-C112.

172. Barlow, P. G., Li, Y., Wilkinson, T. S., Bowdish, D. M. E., Lau, Y. E., Cosseau, C., Haslett, C., Simpson, A. J., Hancock, R. E. W., \& Davidson, D. J. (2006). The human cationic host defense peptide LL-37 mediates contrasting effects on 
apoptotic pathways in different primary cells of the innate immune system. Journal of Leukocyte Biology, 80, 509-520.

173. Zhang, Z., Cherryholmes, G., \& Shively, J. E. (2008). Neutrophil secondary necrosis is induced by LL-37 derived from cathelicidin. Journal of Leukocyte Biology, 84, 780-788.

174. Säll, J., Carlsson, M., Gidlöf, O., Holm, A., Humlén, J., Öhman, J., Svensson, D., Nilsson, B.-O., \& Jönsson, D. (2013). The antimicrobial peptide LL-37 alters human osteoblast $\mathrm{Ca}^{2+}$ handling and induces $\mathrm{Ca}^{2+}$-independent apoptosis. Journal of Innate Immunity, 5, 290-300.

175. Nijnik, A., \& Hancock, R. E. W. (2009). The roles of cathelicidin LL-37 in immune defences and novel clinical applications. Current Opinion in Hematology, 16, 41-47.

176. Ruan, Y., Shen, T., Wang, Y., Hou, M., Li, J., \& Sun, T. (2013). Antimicrobial peptide LL-37 attenuates LTA induced inflammatory effect in macrophages. International Immunopharmacology, 15, 575-580.

177. Larrick, J. W., Hirata, M., Balint, R. F., Lee, J., Zhong, J., \& Wright, S. C. (1995). Human CAP18: A novel antimicrobial lipopolysaccharide-binding protein. Infection and Immunity, 63, 1291-1297.

178. Mookherjee, N., Brown, K. L., Bowdish, D. M. E., Doria, S., Falsafi, R., Hokamp, K., Roche, F. M., Mu, R., Doho, G. H., Pistolic, J., Powers, J.-P., Bryan, J., Brinkman, F. S. L., \& Hancock, R. E. W. (2006). Modulation of the TLR-mediated inflammatory response by the endogenous human host defense peptide LL-37. The Journal of Immunology, 176, 2455-2464.

179. Heilborn, J. D., Nilsson, M. F., Kratz, G., Weber, G., Sørensen, O., Borregaard, N., \& Stahle-Backdahl, M. (2003). The cathelicidin anti-microbial peptide LL37 is involved in re-epithelialization of human skin wounds and is lacking in chronic ulcer epithelium. Journal of Investigative Dermatology, 120, 379-389.

180. Koczulla, R., von Degenfeld, G., Kupatt, C., Krotz, F., Zahler, S., Gloe, T., Issbrucker, K., Unterberger, P., Zaiou, M., Lebherz, C., Karl, A., Raake, P., Pfosser, A., Boekstegers, P., Welsch, U., Hiemstra, P. S., Vogelmeier, C., Gallo, R. L., Clauss, M., \& Bals, R. (2003). An angiogenic role for the human peptide antibiotic LL-37/hCAP-18. The Journal of Clinical Investigation, 111, 16651672. 
181. Salvado, M. D., Di Gennaro, A., Lindbom, L., Agerberth, B., \& Haeggström, J. Z. (2013). Cathelicidin LL-37 induces angiogenesis via PGE2-EP3 signaling in endothelial cells, in vivo inhibition by aspirin. Arteriosclerosis, Thrombosis, and Vascular Biology, 33, 1965-1972.

182. Kin, N. W., Chen, Y., Stefanov, E. K., Gallo, R. L., \& Kearney, J. F. (2011). Cathelin-related antimicrobial peptide differentially regulates $\mathrm{T}$ - and B-cell function. European Journal of Immunology, 41, 3006-3016.

183. Gallo, R. L., Ono, M., Povsic, T., Page, C., Eriksson, E., Klagsbrun, M., \& Bernfield, M. (1994). Syndecans, cell surface heparan sulfate proteoglycans, are induced by a proline-rich antimicrobial peptide from wounds. Proceedings of the National Academy of Sciences of the United States of America, 91, 1103511039.

184. Li, J., Post, M., Volk, R., Gao, Y., Li, M., Metais, C., Sato, K., Tsai, J., Aird, W., Rosenberg, R. D., Hampton, T. G., Sellke, F., Carmeliet, P., \& Simons, M. (2000). PR39, a peptide regulator of angiogenesis. Nature Medicine, 6, 49-55.

185. Ramanathan, B., Wu, H., Ross, C. R., \& Blecha, F. (2004). PR-39, a porcine antimicrobial peptide, inhibits apoptosis: Involvement of caspase-3. Developmental and Comparative Immunology, 28, 163-169.

186. Wu, J., Parungo, C., Wu, G., Kang, P. M., Laham, R. J., Sellke, F. W., Simons, M., \& Li, J. (2004). PR39 inhibits apoptosis in hypoxic endothelial cells: Role of inhibitor apoptosis protein-2. Circulation, 109, 1660-1667.

187. Xiao, Y., Herrera, A. I., Bommineni, Y. R., Soulages, J. L., Prakash, O., \& Zhang, G. (2009). The central kink region of fowlicidin-2, an $\alpha$-helical host defense peptide, is critically involved in bacterial killing and endotoxin neutralization. Journal of Innate Immunity, 1, 268-280.

188. van Dijk, A., Molhoek, E. M., Veldhuizen, E. J. A., Bokhoven, J. L. M. T.-v., Wagendorp, E., Bikker, F., \& Haagsman, H. P. (2009). Identification of chicken cathelicidin-2 core elements involved in antibacterial and immunomodulatory activities. Molecular Immunology, 46, 2465-2473.

189. Wei, L., Yang, J., He, X., Mo, G., Hong, J., Yan, X., Lin, D., \& Lai, R. (2013). Structure and function of a potent lipopolysaccharide-binding antimicrobial and anti-inflammatory peptide. Journal of Medicinal Chemistry, 56, 3546-3556. 
190. Buck, M. A., Olah, T. A., Weitzmann, C. J., \& Cooperman, B. S. (1989). Protein estimation by the product of integrated peak area and flow rate. Analytical Biochemistry, 182, 295-299.

191. The, T., \& Feltkamp, T. (1970). Conjugation of fluorescein isothiocyanate to antibodies: I. Experiments on the conditions of conjugation. Immunology, 18, 865.

192. Smith, P., Krohn, R. I., Hermanson, G., Mallia, A., Gartner, F., Provenzano, M., Fujimoto, E., Goeke, N., Olson, B., \& Klenk, D. (1985). Measurement of protein using bicinchoninic acid. Analytical Biochemistry, 150, 76-85.

193. Jorgensen, J. H. (1993). Methods for dilution antimicrobial susceptibility tests for bacteria that grow aerobically: approved standard: NCCLS document M7A3: NCCLS.

194. Quinn, M. T., DeLeo, F. R., \& Bokoch, G. M. (2007). Neutrophil methods and protocols (Vol. 412): Springer.

195. Quade, M. J., \& Roth, J. A. (1997). A rapid, direct assay to measure degranulation of bovine neutrophil primary granules. Veterinary Immunology and Immunopathology, 58, 239-248.

196. Gennaro, R., Scocchi, M., Merluzzi, L., \& Zanetti, M. (1998). Biological characterization of a novel mammalian antimicrobial peptide. Biochimica et Biophysica Acta - General Subjects, 1425, 361-368.

197. Coomber, B. L., Galligan, C. L., \& Gentry, P. A. (1997). Comparison of in vitro function of neutrophils from cattle deficient in plasma factor XI activity and from normal animals. Veterinary Immunology and Immunopathology, 58, 121131.

198. Tan, A. S., \& Berridge, M. V. (2000). Superoxide produced by activated neutrophils efficiently reduces the tetrazolium salt, WST-1 to produce a soluble formazan: A simple colorimetric assay for measuring respiratory burst activation and for screening anti-inflammatory agents. Journal of Immunological Methods, 238, 59-68.

199. Berger, M., O'Shea, J., Cross, A. S., Folks, T. M., Chused, T. M., Brown, E. J., \& Frank, M. (1984). Human neutrophils increase expression of C3bi as well as C3b receptors upon activation. Journal of Clinical Investigation, 74, 1566. 
200. Cooper, J. A. (1987). Effects of cytochalasin and phalloidin on actin. The Journal of Cell Biology, 105, 1473-1478.

201. Prinz, W. A., Åslund, F., Holmgren, A., \& Beckwith, J. (1997). The role of the thioredoxin and glutaredoxin pathways in reducing protein disulfide bonds in the Escherichia coli cytoplasm. Journal of Biological Chemistry, 272, 15661-15667.

202. Villaverde, A., \& Mar Carrió, M. (2003). Protein aggregation in recombinant bacteria: Biological role of inclusion bodies. Biotechnology Letters, 25, 13851395.

203. Suckau, D., Resemann, A., Schuerenberg, M., Hufnagel, P., Franzen, J., \& Holle, A. (2003). A novel MALDI LIFT-TOF/TOF mass spectrometer for proteomics. Analytical and Bioanalytical Chemistry, 376, 952-965.

204. Pappin, D. J. C., Hojrup, P., \& Bleasby, A. J. (1993). Rapid identification of proteins by peptide-mass fingerprinting. Current Biology, 3, 327-332.

205. Marra, M. N., Wilde, C. G., Griffith, J. E., Snable, J. L., \& Scott, R. W. (1990). Bactericidal/permeability-increasing protein has endotoxin-neutralizing activity. Journal of Immunology, 144, 662-666.

206. Wright, S. D., Ramos, R. A., Tobias, P. S., Ulevitch, R. J., \& Mathison, J. C. (1990). CD14, a receptor for complexes of lipopolysaccharide (LPS) and LPS binding protein. Science, 249, 1431-1433.

207. Rawlings, N. D., Morton, F. R., Kok, C. Y., Kong, J., \& Barrett, A. J. (2008). MEROPS: The peptidase database. Nucleic Acids Research, 36, D320-325.

208. Singh, S. M., \& Panda, A. K. (2005). Solubilization and refolding of bacterial inclusion body proteins. Journal of Bioscience and Bioengineering, 99, 303-310.

209. Torrent, M., Pulido, D., Nogués, M. V., \& Boix, E. (2012). Exploring new biological functions of amyloids: Bacteria cell agglutination mediated by host protein aggregation. PLoS Pathogens, 8, e1003005.

210. Brown, G. B., \& Roth, J. A. (1991). Comparison of the response of bovine and human neutrophils to various stimuli. Veterinary Immunology and Immunopathology, 28, 201-218.

211. Altschul, S. F., Gish, W., Miller, W., Myers, E. W., \& Lipman, D. J. (1990). Basic local alignment search tool. Journal of molecular biology, 215, 403-410. 
212. Gordon, Y. J., Romanowski, E. G., \& McDermott, A. M. (2005). A review of antimicrobial peptides and their therapeutic potential as anti-infective drugs. Current Eye Research, 30, 505-515.

213. Ribeiro, M., Franquelim, H. G., Torcato, I. M., Ramu, V. G., Heras, M., Bardaji, E. R., \& Castanho, M. A. (2012). Antimicrobial properties of analgesic kyotorphin peptides unraveled through atomic force microscopy. Biochemical and Biophysical Research Communications, 420, 676-679.

214. Nathan, C. (2006). Neutrophils and immunity: Challenges and opportunities. Nature Reviews Immunology, 6, 173-182.

215. Bals, R., \& Wilson, J. M. (2003). Cathelicidins - A family of multifunctional antimicrobial peptides. Cellular and Molecular Life Sciences, 60, 711-720.

216. Catrina, S.-B., Refai, E., \& Andersson, M. (2009). The cytotoxic effects of the anti-bacterial peptides on leukocytes. Journal of Peptide Science, 15, 842-848.

217. Fenton, M. J., \& Golenbock, D. T. (1998). LPS-binding proteins and receptors. Journal of Leukocyte Biology, 64, 25-32.

218. Iovine, N., Eastvold, J., Elsbach, P., Weiss, J. P., \& Gioannini, T. L. (2002). The carboxyl-terminal domain of closely related endotoxin-binding proteins determines the target of protein-lipopolysaccharide complexes. Journal of Biological Chemistry, 277, 7970-7978.

219. Scott, M. G., Vreugdenhil, A. C. E., Buurman, W. A., Hancock, R. E. W., \& Gold, M. R. (2000). Cutting edge: Cationic antimicrobial peptides block the binding of lipopolysaccharide (LPS) to LPS binding protein. The Journal of Immunology, 164, 549-553.

220. Zughaier, S. M., Shafer, W. M., \& Stephens, D. S. (2005). Antimicrobial peptides and endotoxin inhibit cytokine and nitric oxide release but amplify respiratory burst response in human and murine macrophages. Cellular Microbiology, 7, 1251-1262.

221. Storici, P., Tossi, A., Lenarčič, B., \& Romeo, D. (1996). Purification and structural characterization of bovine cathelicidins, precursors of antimicrobial peptides. European Journal of Biochemistry, 238, 769-776.

222. Wu, M., \& Hancock, R. E. W. (1999). Interaction of the cyclic antimicrobial cationic peptide bactenecin with the outer and cytoplasmic membrane. Journal of Biological Chemistry, 274, 29-35. 
223. Scocchi, M., Tossi, A., \& Gennaro, R. (2011). Proline-rich antimicrobial peptides: Converging to a non-lytic mechanism of action. Cellular and Molecular Life Sciences, 68, 2317-2330.

224. Ahmad, A., Asthana, N., Azmi, S., Srivastava, R. M., Pandey, B. K., Yadav, V., \& Ghosh, J. K. (2009). Structure-function study of cathelicidin-derived bovine antimicrobial peptide BMAP-28: Design of its cell-selective analogs by amino acid substitutions in the heptad repeat sequences. Biochimica et Biophysica Acta (BBA) - Biomembranes, 1788, 2411-2420.

225. Dore, M., Slauson, D. O., Suyemoto, M. M., \& Neilsen, N. R. (1990). Calcium mobilization in C5a-stimulated adult and newborn bovine neutrophils. Inflammation, 14, 71-82.

226. Salmon, M. D., \& Ahluwalia, J. (2011). Pharmacology of receptor operated calcium entry in human neutrophils. International Immunopharmacology, 11, 145-148.

227. Faurschou, M., \& Borregaard, N. (2003). Neutrophil granules and secretory vesicles in inflammation. Microbes and Infection, 5, 1317-1327.

228. Cai, T. Q., Weston, P. G., Lund, L. A., Brodie, B., McKenna, D. J., \& Wagner, W. C. (1994). Association between neutrophil functions and periparturient disorders in cows. American Journal of Veterinary Research, 55, 934-943.

229. Klink, M., Bednarska, K., Blus, E., Kielbik, M., \& Sulowska, Z. (2012). Seasonal changes in activities of human neutrophils in vitro. Inflammation Research, 61, 11-16.

230. Nelson, R. J. (2004). Seasonal immune function and sickness responses. Trends in Immunology, 25, 187-192.

231. Turner, J., Cho, Y., Dinh, N.-N., Waring, A. J., \& Lehrer, R. I. (1998). Activities of LL-37, a cathelin-associated antimicrobial peptide of human neutrophils. Antimicrobial Agents and Chemotherapy, 42, 2206-2214.

232. Cassatella, M. A. (1995). The production of cytokines by polymorphonuclear neutrophils. Immunology Today, 16, 21-26.

233. Bokoch, G. (1995). Chemoattractant signaling and leukocyte activation. Blood, 86, 1649-1660.

234. Subbalakshmi, C., \& Sitaram, N. (1998). Mechanism of antimicrobial action of indolicidin. FEMS Microbiology Letters, 160, 91-96. 
235. Lee, J. Y., Yang, S. T., Kim, H. J., Lee, S. K., Jung, H. H., Shin, S. Y., \& Kim, J. I. (2009). Different modes of antibiotic action of homodimeric and monomeric bactenecin, a cathelicidin-derived antibacterial peptide. Biochemistry and Molecular Biology Reports, 42, 586-592.

236. Witko-Sarsat, V., Rieu, P., Descamps-Latscha, B., Lesavre, P., \& HalbwachsMecarelli, L. (2000). Neutrophils: Molecules, functions and pathophysiological aspects. Laboratory Investigation, 80, 617-654.

237. Ye, R. D. (2010). Biased agonism in chemoattractant receptor signaling. Journal of Leukocyte Biology, 87, 959-961.

238. Stie, J., \& Jesaitis, A. J. (2007). Reorganization of the human neutrophil plasma membrane is associated with functional priming: Implications for neutrophil preparations. Journal of Leukocyte Biology, 81, 672-685.

239. Zamzami, N., Marchetti, P., Castedo, M., Decaudin, D., Macho, A., Hirsch, T., Susin, S. A., Petit, P. X., Mignotte, B., \& Kroemer, G. (1995). Sequential reduction of mitochondrial transmembrane potential and generation of reactive oxygen species in early programmed cell death. Journal of Experimental Medicine, 182, 367-377.

240. Kellogg, T. A., Weiss Iii, C. A., Johnston, J. W., Wasiluk, K. R., \& Dunn, D. L. (1999). Antiendotoxin agents share molecular homology within their lipopolysaccharide binding domains. Journal of Surgical Research, 85, 136-141.

241. Ghiselli, R., Giacometti, A., Cirioni, O., Circo, R., Mocchegiani, F., Skerlavaj, B., D'Amato, G., Scalise, G., Zanetti, M., \& Saba, V. (2003). Neutralization of endotoxin in vitro and in vivo by BAC7(1-35), a proline-rich antibacterial peptide. Shock, 19, 577-581.

242. Giacometti, A., Cirioni, O., Ghiselli, R., Mocchegiani, F., D'Amato, G., Circo, R., Orlando, F., Skerlavaj, B., Silvestri, C., Saba, V., Zanetti, M., \& Scalise, G. (2004). Cathelicidin peptide sheep myeloid antimicrobial peptide-29 prevents endotoxin-induced mortality in rat models of septic shock. American Journal of Respiratory and Critical Care Medicine, 169, 187-194.

243. Falla, T. J., Nedra Karunaratne, D., \& Hancock, R. E. W. (1996). Mode of action of the antimicrobial peptide indolicidin. Journal of Biological Chemistry, 271, 19298-19303. 
244. Nagpal, S., Kaur, K. J., Jain, D., \& Salunke, D. M. (2002). Plasticity in structure and interactions is critical for the action of indolicidin, an antibacterial peptide of innate immune origin. Protein Science, 11, 2158-2167.

245. Okuda, D., Yomogida, S., Tamura, H., \& Nagaoka, I. (2006). Determination of the antibacterial and lipopolysaccharide-neutralizing regions of guinea pig neutrophil cathelicidin peptide CAP11. Antimicrobial Agents and Chemotherapy, 50, 2602-2607.

246. Nicolas, P. (2009). Multifunctional host defense peptides: Intracellular-targeting antimicrobial peptides. FEBS Journal, 276, 6483-6496.

247. Tomasinsig, L., Benincasa, M., Scocchi, M., Skerlavaj, B., Tossi, A., Zanetti, M., \& Gennaro, R. (2010). Role of cathelicidin peptides in bovine host defense and healing. Probiotics and Antimicrobial Proteins, 2, 12-20.

248. Tomasinsig, L., Skerlavaj, B., Papo, N., Giabbai, B., Shai, Y., \& Zanetti, M. (2006). Mechanistic and functional studies of the interaction of a proline-rich antimicrobial peptide with mammalian cells. Journal of Biological Chemistry, 281, 383-391.

249. Kay, B. K., Williamson, M. P., \& Sudol, M. (2000). The importance of being proline: The interaction of proline-rich motifs in signaling proteins with their cognate domains. The FASEB journal, 14, 231-241.

250. Williamson, M. P. (1994). The structure and function of proline-rich regions in proteins. Biochemical Journal, 297, 249.

251. Li, J.-M., Mullen, A. M., Yun, S., Wientjes, F., Brouns, G. Y., Thrasher, A. J., \& Shah, A. M. (2002). Essential role of the NADPH oxidase subunit $\mathrm{p} 47^{\text {phox }}$ in endothelial cell superoxide production in response to phorbol ester and tumor necrosis factor- $\alpha$. Circulation Research, 90, 143-150.

252. Bokoch, G. M., \& Diebold, B. A. (2002). Current molecular models for NADPH oxidase regulation by Rac GTPase. Blood, 100, 2692-2695.

253. Huntington, J. A., Read, R. J., \& Carrell, R. W. (2000). Structure of a serpinprotease complex shows inhibition by deformation. Nature, 407, 923-926.

254. Silverman, G. A., Bird, P. I., Carrell, R. W., Church, F. C., Coughlin, P. B., Gettins, P. G. W., Irving, J. A., Lomas, D. A., Luke, C. J., Moyer, R. W., Pemberton, P. A., Remold-O'Donnell, E., Salvesen, G. S., Travis, J., \& Whisstock, J. C. (2001). The Serpins Are an Expanding Superfamily of 
Structurally Similar but Functionally Diverse Proteins: EVOLUTION, MECHANISM OF INHIBITION, NOVEL FUNCTIONS, AND A REVISED NOMENCLATURE. Journal of Biological Chemistry, 276, 33293-33296.

255. Theilgaard-Mönch, K., Jacobsen, L. C., Nielsen, M. J., Rasmussen, T., Udby, L., Gharib, M., Arkwright, P. D., Gombart, A. F., Calafat, J., \& Moestrup, S. K. (2006). Haptoglobin is synthesized during granulocyte differentiation, stored in specific granules, and released by neutrophils in response to activation. Blood, 108, 353-361.

256. Langlois, M. R., \& Delanghe, J. R. (1996). Biological and clinical significance of haptoglobin polymorphism in humans. Clinical Chemistry, 42, 1589-1600.

257. Pantaloni, D., Le Clainche, C., \& Carlier, M.-F. (2001). Mechanism of actinbased motility. Science, 292, 1502-1506.

258. Pollard, T. D., \& Borisy, G. G. (2003). Cellular motility driven by assembly and disassembly of actin filaments. Cell, 112, 453-465.

259. May, R. C., \& Machesky, L. M. (2001). Phagocytosis and the actin cytoskeleton. Journal of Cell Science, 114, 1061-1077.

260. Tamura, M., Kai, T., Tsunawaki, S., Lambeth, J. D., \& Kameda, K. (2000). Direct interaction of actin with $\mathrm{p} 47^{\text {phox }}$ of neutrophil NADPH oxidase. Biochemical and Biophysical Research Communications, 276, 1186-1190.

261. Tamura, M., Kanno, M., \& Endo, Y. (2000). Deactivation of neutrophil NADPH oxidase by actin-depolymerizing agents in a cell-free system. Biochemical Journal, 349, 369-375.

262. Simard, J. C., Simon, M. M., Tessier, P. A., \& Girard, D. (2011). Damageassociated molecular pattern S100A9 increases bactericidal activity of human neutrophils by enhancing phagocytosis. Journal of Immunology, 186, 36223631.

263. Kerkhoff, C., Nacken, W., Benedyk, M., Dagher, M. C., Sopalla, C., \& Doussiere, J. (2005). The arachidonic acid-binding protein S100A8/A9 promotes NADPH oxidase activation by interaction with $\mathrm{p} 67^{\text {phox }}$ and Rac-2. The FASEB journal, 19, 467-469.

264. Graille, M., Stura, E. A., Corper, A. L., Sutton, B. J., Taussig, M. J., Charbonnier, J.-B., \& Silverman, G. J. (2000). Crystal structure of a Staphylococcus aureus protein A domain complexed with the Fab fragment of a 
human IgM antibody: Structural basis for recognition of B-cell receptors and superantigen activity. Proceedings of the National Academy of Sciences, 97, 5399-5404.

265. Faulstich, H., Trischmann, H., \& Mayer, D. (1983). Preparation of tetramethylrhodaminyl-phalloidin and uptake of the toxin into short-term cultured hepatocytes by endocytosis. Experimental Cell Research, 144, 73-82.

266. Li, X. J., Marchal, C. C., Stull, N. D., Stahelin, R. V., \& Dinauer, M. C. (2010). p $47^{\text {phox }}$ Phox homology domain regulates plasma membrane but not phagosome neutrophil NADPH oxidase activation. Journal of Biological Chemistry, 285, 35169-35179.

267. Li, J.-M., \& Shah, A. M. (2003). Mechanism of endothelial cell NADPH oxidase activation by angiotensin II: Role of the $\mathrm{p} 47^{\text {phox }}$ subunit. Journal of Biological Chemistry, 278, 12094-12100.

268. Li, J.-M., Wheatcroft, S., Fan, L. M., Kearney, M. T., \& Shah, A. M. (2004). Opposing roles of $\mathrm{p} 47^{\text {phox }}$ in basal versus angiotensin II-stimulated alterations in vascular $\mathrm{O}^{2-}$ production, vascular tone, and mitogen-activated protein kinase activation. Circulation, 109, 1307-1313.

269. Tamura, M., Itoh, K., Akita, H., Takano, K., \& Oku, S. (2006). Identification of an actin-binding site in $\mathrm{p} 47^{\text {phox }}$ an organizer protein of NADPH oxidase. FEBS Letters, 580, 261-267.

270. Liu, Y., Fiskum, G., \& Schubert, D. (2002). Generation of reactive oxygen species by the mitochondrial electron transport chain. Journal of Neurochemistry, 80, 780-787.

271. Thrasher, A. J. (2002). Wasp in immune-system organization and function. Nature Reviews Immunology, 2, 635-646.

272. Miles, K., Clarke, D. J., Lu, W., Sibinska, Z., Beaumont, P. E., Davidson, D. J., Barr, T. A., Campopiano, D. J., \& Gray, M. (2009). Dying and necrotic neutrophils are anti-inflammatory secondary to the release of $\alpha$-defensins. The Journal of Immunology, 183, 2122-2132.

273. Silva, M. T. (2011). Macrophage phagocytosis of neutrophils at inflammatory/infectious foci: A cooperative mechanism in the control of infection and infectious inflammation. Journal of Leukocyte Biology, 89, 675683. 
274. Bergdoll, M., Remy, M. H., Cagnon, C., Masson, J. M., \& Dumas, P. (1997). Proline-dependent oligomerization with arm exchange. Structure, 5, 391-401.

275. Hall, A. (1998). Rho GTPases and the actin cytoskeleton. Science, 279, 509-514.

276. Sohn, R. H., \& Goldschmidt-Clermont, P. J. (1994). Profilin: At the crossroads of signal transduction and the actin cytoskeleton. BioEssays, 16, 465-472.

277. Sweeney, S. E., \& Kim, Y. B. (2004). Identification of a novel Fc $\gamma$ RIIIa $\alpha-$ associated molecule that contains significant homology to porcine cathelin. The Journal of Immunology, 172, 1203-1212. 


\section{Appendix 1: Bovine AMP Interactions with Cytokine Antibodies}

ELISAs measuring bovine cytokines were intended for use in the assessment of cytokine release by isolated neutrophils treated with bovine cathelicidin AMP (Chapter 4, section 4.3.5). However, the bovine cathelicidin AMPs appeared to interfere or bind with ELISA antibodies, generating false-positive measurements, as illustrated by the supporting experimental evidence below.

\section{Interaction with ELISA antibodies}

To determine the extent of the false-positive reading, individual bovine AMPs (Bac1, linear Bac1, Bac5, indolicidin, BMAP-28, BMAP-34, LL-37) were diluted to $5 \mu \mathrm{M}$ in culture media and tested in bovine-specific IL-1 $\beta$, IL-6 and IL-10 ELISAs (Figure A1.1 A). Strong absorbance readings were measured for linear Bac1, BMAP-28 and BMAP-34 across all three ELISAs. LL-37 produced a modest absorbance measurement in the IL-6 and IL-10 ELISAs in contrast to the two bovine $\alpha$-helical peptides.

To determine the origin of the false-positive signal, reagents of the IL-1 $\beta$ ELISA were systematically removed (Figure A1.1 B). Through this technique the potential for false positives due to a non-specific AMP interaction with TMB and streptavidin-HRP were eliminated (Figure A1.1 B (ii), (iii)). By immobilising the AMPs directly on the ELISA plate, blocking, and then incubating with detection antibody, streptavidin-HRP and TMB as normal, it was possible to show that the detection antibody bound to immobilised AMP (Figure A1.1 B (iv)). However, omitting the capture antibody and blocking the plate so as to remove all possible peptide binding sites resulted in absorbance measurements equivalent to the standard procedure (Figure A1.1 B (v)). 
A

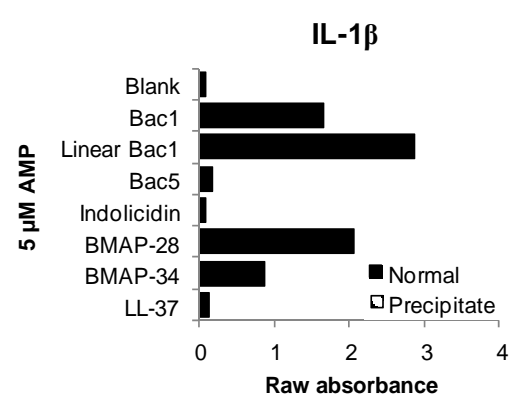

IL-6

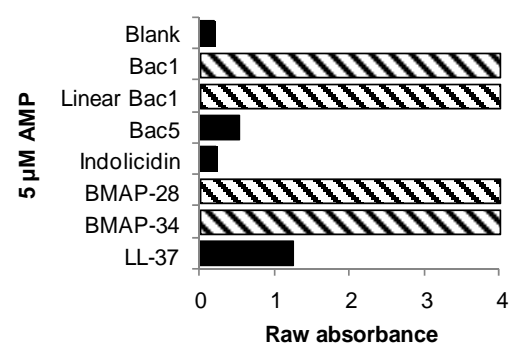

IL-10

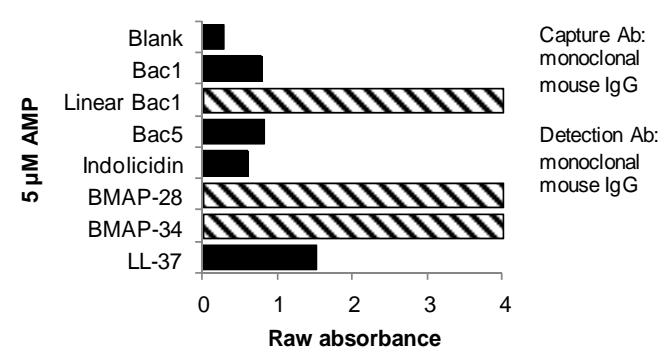

B

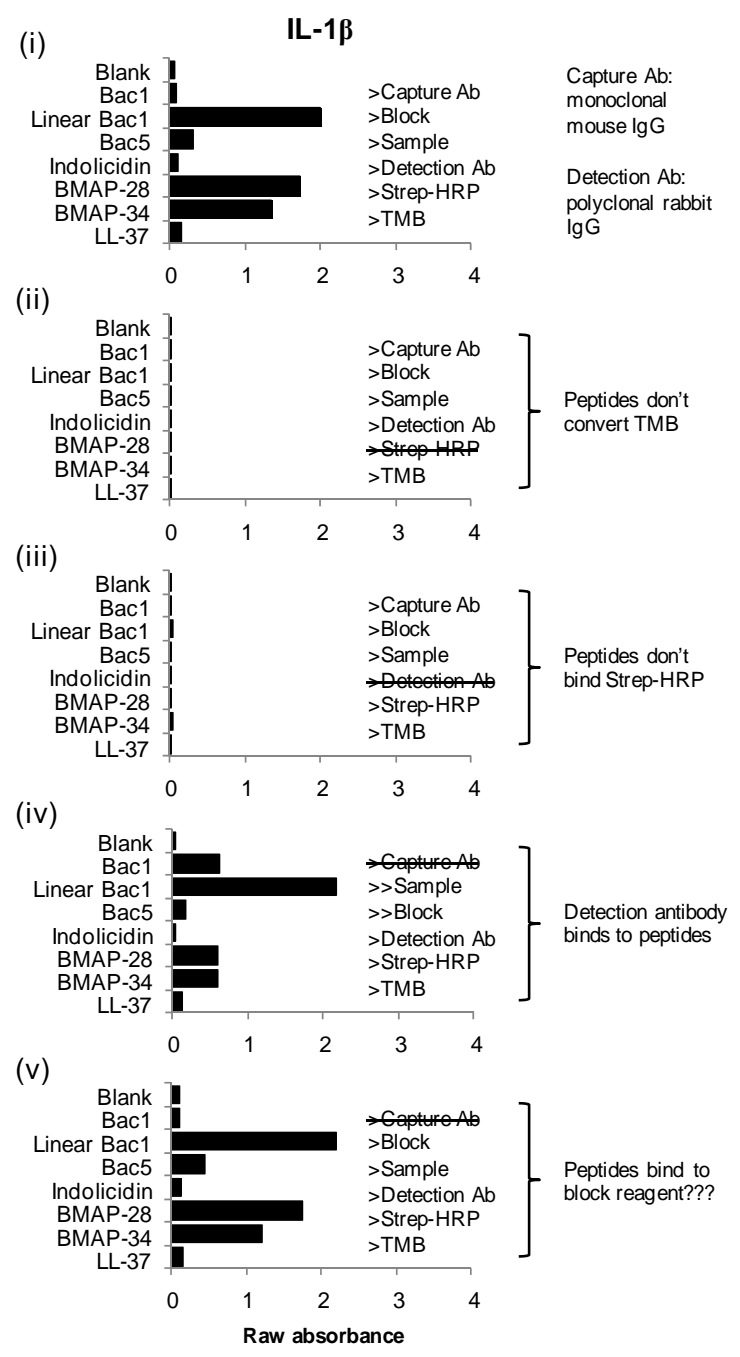

\section{Figure A1.1 Analysis of false-positives in bovine cytokine ELISAs}

A standard ELISA procedure was used to determine the extent of false-positive signal in control samples containing only $5 \mu \mathrm{M}$ cathelicidin AMP and RPMI 1640 medium. Maxisorp 96-well plates (Nunc) were coated with capture antibody overnight at room temperature. Between each step the plates were washed three times with wash solution A (Appendix 2). The plates were blocked with wash solution A containing $1 \%(w / v)$ BSA $(66.5 \mathrm{kDa})$ for 2 hour at room temperature. Samples were incubated for 2 hours at room temperature. Detection antibody was incubated for 1 hour at room temperature. Streptavidin-HRP was incubated for 30 minutes at room temperature. TMB substrate was added for 30 minutes at room temperature or until the blue colour developed sufficiently. The reaction was then stopped with an equal volume of $1 \mathrm{M} \mathrm{H}_{2} \mathrm{SO}_{4}$. Absorbance was measured at $450 \mathrm{~nm}$ using a Synergy 2 multi-mode microplate reader and Gen5 software. (A) Standard ELISA procedure for IL-1 $\beta$, IL-6 and IL-10 antibodies. Bars with diagonal lines indicate samples in which TMB conversion was so complete that insoluble precipitates formed which affected absorbance readings. Data represent a single experiment performed in duplicate. (B) IL-1 $\beta$ ELISA reagents were systematically removed to determine the origin of the false-positive signal. (i) Standard procedure. (ii) Streptavidin-HRP was omitted to determine if the AMPs directly 
converted TMB substrate to the blue product. (iii) The detection antibody was omitted to determine if streptavidin-HRP bound to the AMPs. (iv) The capture antibody was omitted and the AMP samples were used to coat the ELISA plates at room temperature overnight, the standard procedure was then followed to determine if the detection antibody bound to the AMPs. (v) The capture antibody was omitted from the standard procedure to determine if the AMPs bound to the block reagent. Data represent a single experiment performed in duplicate. Ab, antibody.

These results indicated that BMAP-28 and BMAP-34 were able to bind to the blocking reagent as well as the antibodies (due to the difference observed between Figure A1.1 B, (iv) and (v)). Removal of the capture antibody did not affect the absorbance of samples containing linear Bac1, where it was observed, therefore it was possible that linear Bac1 was able to bind in the spaces between blocking reagent molecules and establish a bond with detection antibody molecules. For these reasons several different blocking reagents were employed to test whether the type of blocking reagent affected the AMP-antibody interaction. However, neither $2 \%(\mathrm{w} / \mathrm{v})$ polyvinylpyrrolidone $(44 \mathrm{kDa}, \mathrm{BDH}$ Chemicals), $1 \%(\mathrm{w} / \mathrm{v})$ polyvinyl alcohol (10 kDa, Sigma-Aldrich) or $1 \%(\mathrm{w} / \mathrm{v})$ ficoll (400 kDa, Sigma-Aldrich) made a significant difference to any of the peptides generating the greatest false-positive readings (Figure A1.2).
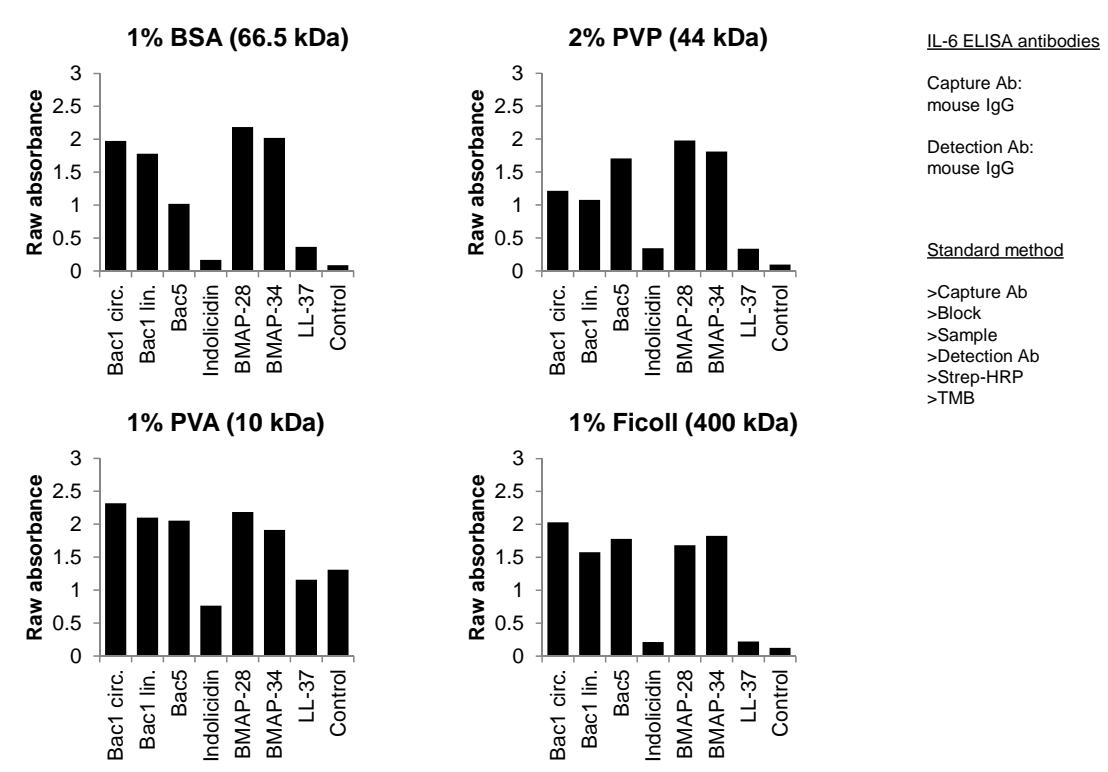

\section{Figure A1.2 Test of ELISA blocking reagents}

The IL-6 ELISA antibody pairs were used to test the effectiveness of different blocking reagents in reducing the AMP-antibody interaction. A standard ELISA method was used. Blocking reagent containing $1 \%(\mathrm{w} / \mathrm{v})$ BSA is provided for comparison with $2 \%(\mathrm{w} / \mathrm{v})$ polyvinylpyrrolidone, $1 \%(\mathrm{w} / \mathrm{v})$ polyvinyl alcohol and 1\% (w/v) ficoll. Data represent a single experiment performed in duplicate. 


\section{Significance of the AMP-antibody interactions}

Antibodies perform a number of important functions during infection such as opsonisation of pathogens and activation of complement. As it is unclear as to which part of the antibody molecule the AMPs might bind to, it is difficult to ascertain how the interaction may affect regular antibody activity. Several possibilities are presented here.

The AMP-antibody interaction may enhance antibody function. Given the ability of the bovine $\alpha$-helical AMPs to interact with bacterial membranes and different IgG molecules, the bovine $\alpha$-helical AMPs may act as adapter molecules to allow nonspecific IgG opsonisation of bacteria. This would improve complement activation and clearance by phagocytes without requiring specific antigen-antibody recognition. Coincidently, a protein with significant homology to porcine cathelin and the CLD of cathelicidins has been found to associate with the porcine IgG receptor Fc $\gamma$ RIIIa $\alpha$ [277]. This provides some evidence for a purposeful interaction between cathelicidins, antibody and antibody recognition. Hence, the AMP domain may somehow enhance receptor activation upon antibody recognition, thereby promoting bacterial clearance.

Conversely, the AMP-antibody interaction may inhibit antibody function. The AMPs may non-specifically bind to the variable region of antibody molecules, thereby preventing antigen recognition and regulating immune responses. Alternatively they may bind to the constant region of antibody and prevent Fc receptor recognition of antibody-antigen complexes and likewise regulate immune responses.

The AMP-antibody interaction could also possibly act to remove AMP from the inflammatory environment during the transition to the adaptive immune response. This would prevent re-activation of proinflammatory functions in immune cells when the cathelicidin concentration returns to low levels.

Finally, the interaction may be transient in the inflammatory environment and may not result in changes to the function of either molecule. Despite all these possibilities, unforeseen interactions between AMPs and assay components, particularly antibody, should be carefully controlled against in assays which contain free AMP. Future research may clarify the significance of these cathelicidin AMP-antibody interactions. 


\section{Appendix 2: Solutions and Reagents}

All solutions were made in $\mathrm{MQ} \cdot \mathrm{H}_{2} \mathrm{O}$ to the required volume unless otherwise indicated.

\section{General solutions}

\subsection{PBS}

\begin{tabular}{|lll|}
\hline Item & Concentration & Supplier \\
\hline $\mathrm{NaCl}$ & $137 \mathrm{mM}$ & Thermo Fisher Scientific New Zealand Ltd. \\
$\mathrm{KCl}$ & $2.7 \mathrm{mM}$ & Thermo Fisher Scientific New Zealand Ltd. \\
$\mathrm{Na}_{2} \mathrm{HPO}_{4}$ & $10 \mathrm{mM}$ & Thermo Fisher Scientific New Zealand Ltd. \\
$\mathrm{NaH}_{2} \mathrm{PO}_{4}$ & $1.8 \mathrm{mM}$ & Fisher Scientific, Loughborough, UK \\
\hline
\end{tabular}

Adjust $\mathrm{pH}$ to 7.4 using $\mathrm{NaOH}$ or $\mathrm{HCl}$. Autoclave to sterilise. Store at room temperature.

\subsection{TBS}

\begin{tabular}{|lll|}
\hline Item & Concentration & Supplier \\
\hline Tris & $50 \mathrm{mM}$ & Fisher BioReagents, NJ, USA \\
$\mathrm{NaCl}$ & $150 \mathrm{mM}$ & Thermo Fisher Scientific New Zealand Ltd. \\
\hline
\end{tabular}

Adjust $\mathrm{pH}$ to 7.5 using $\mathrm{HCl}$. Autoclave to sterilise. Store at room temperature. 


\section{Molecular biology solutions}

\subsection{Solutions for protein expression}

\subsubsection{LB/kan/tet/amp}

\begin{tabular}{|lll|}
\hline Item & Concentration & Supplier \\
\hline Luria Broth & $20 \mathrm{mg} / \mathrm{ml}$ & Life Technologies Inc. \\
Kanamycin & $15 \mu \mathrm{g} / \mathrm{ml}$ & Sigma-Aldrich \\
Tetracycline & $12.5 \mu \mathrm{g} / \mathrm{ml}$ & Sigma-Aldrich \\
Ampicillin & $50 \mu \mathrm{g} / \mathrm{ml}$ & Applichem GmbH, Darmstadt, Germany \\
Glucose & $0.1 \%(\mathrm{w} / \mathrm{v})$ & Sigma-Aldrich \\
\hline
\end{tabular}

Autoclave to sterilise. Store at $4{ }^{\circ} \mathrm{C}$.

\subsubsection{LB/kan/tet/amp agar}

\begin{tabular}{|lll|}
\hline Item & Concentration & Supplier \\
\hline Luria Broth & $20 \mathrm{mg} / \mathrm{ml}$ & Life Technologies Inc. \\
Luria Broth Agar & $15 \mathrm{mg} / \mathrm{ml}$ & Life Technologies Inc. \\
Kanamycin & $15 \mu \mathrm{g} / \mathrm{ml}$ & Sigma-Aldrich \\
Tetracycline & $12.5 \mu \mathrm{g} / \mathrm{ml}$ & Applichem GmbH \\
Ampicillin & $50 \mu \mathrm{glml}$ & Sigma-Aldrich \\
\hline
\end{tabular}

Autoclave to sterilise. Store at $4{ }^{\circ} \mathrm{C}$.

\subsection{Solutions for protein purification}

\subsubsection{Column stripping solution}

\begin{tabular}{|lll|}
\hline Item & Concentration & Supplier \\
\hline EDTA & $50 \mathrm{mM}$ & Thermo Fisher Scientific New Zealand Ltd. \\
$\mathrm{Na}_{2} \mathrm{HPO}_{4}$ & $20 \mathrm{mM}$ & Thermo Fisher Scientific New Zealand Ltd. \\
$\mathrm{NaCl}$ & $500 \mathrm{mM}$ & Thermo Fisher Scientific New Zealand Ltd. \\
\hline
\end{tabular}

Filter through $0.2 \mu \mathrm{m}$ membrane (Minisart, Sartorius). Store at room temperature. 


\subsubsection{Nickel loading solution}

\begin{tabular}{|lll|}
\hline Item & Concentration & Supplier \\
\hline $\mathrm{NiSO}_{4}$ & $100 \mathrm{mM}$ & Sigma-Aldrich \\
\hline
\end{tabular}

Filter through $0.2 \mu \mathrm{m}$ membrane. Store at room temperature.

\subsubsection{Column storage solution}

\begin{tabular}{|lll|}
\hline Item & Concentration & Supplier \\
\hline Ethanol & $20 \%(\mathrm{v} / \mathrm{v})$ & Thermo Fisher Scientific New Zealand Ltd. \\
\hline
\end{tabular}

Filter through $0.2 \mu \mathrm{m}$ membrane. Store at room temperature.

\subsubsection{5× TE}

\begin{tabular}{|lll|}
\hline Item & Concentration & Supplier \\
\hline Tris & $50 \mathrm{mM}$ & Fisher BioReagents, NJ, USA \\
EDTA & $5 \mathrm{mM}$ & Thermo Fisher Scientific New Zealand Ltd. \\
\hline
\end{tabular}

Adjust $\mathrm{pH}$ to 8.0 with $\mathrm{HCl}$. Filter through $0.2 \mu \mathrm{m}$ membrane. Store at room temperature.

\subsubsection{Lysis buffer A}

\begin{tabular}{|lll|}
\hline Item & Concentration & Supplier \\
\hline $\mathrm{NaCl}$ & $500 \mathrm{mM}$ & Thermo Fisher Scientific New Zealand Ltd. \\
$\mathrm{NaH}_{2} \mathrm{PO}_{4}$ & $29 \mathrm{mM}$ & Thermo Fisher Scientific New Zealand Ltd. \\
$\mathrm{Na}_{2} \mathrm{HPO}_{4}$ & $471 \mathrm{mM}$ & Fisher Scientific \\
Imidazole & $10 \mathrm{mM}$ & JT Baker Chemical Co., NJ, USA \\
\hline
\end{tabular}

Adjust $\mathrm{pH}$ to 7.4 with $\mathrm{NaOH}$ or $\mathrm{HCl}$. Filter through $0.2 \mu \mathrm{m}$ membrane. Store at room temperature.

\subsubsection{Wash buffer A}

\begin{tabular}{|lll|}
\hline Item & Concentration & Supplier \\
\hline $\mathrm{NaCl}$ & $500 \mathrm{mM}$ & Thermo Fisher Scientific New Zealand Ltd. \\
$\mathrm{NaH}_{2} \mathrm{PO}_{4}$ & $365 \mathrm{mM}$ & Thermo Fisher Scientific New Zealand Ltd. \\
$\mathrm{Na}_{2} \mathrm{HPO}_{4}$ & $135 \mathrm{mM}$ & Fisher Scientific \\
Imidazole & $20 \mathrm{mM}$ & JT Baker Chemical Co. \\
\hline
\end{tabular}

Adjust $\mathrm{pH}$ to 7.4 with $\mathrm{NaOH}$ or $\mathrm{HCl}$. Filter through $0.2 \mu \mathrm{m}$ membrane. Store at room temperature. 


\subsubsection{Elution buffer A}

\begin{tabular}{|lll|}
\hline Item & Concentration & Supplier \\
\hline $\mathrm{NaCl}$ & $500 \mathrm{mM}$ & Thermo Fisher Scientific New Zealand Ltd. \\
$\mathrm{NaH}_{2} \mathrm{PO}_{4}$ & $365 \mathrm{mM}$ & Thermo Fisher Scientific New Zealand Ltd. \\
$\mathrm{Na}_{2} \mathrm{HPO}_{4}$ & $135 \mathrm{mM}$ & Fisher Scientific \\
Imidazole & $500 \mathrm{mM}$ & JT Baker Chemical Co. \\
\hline
\end{tabular}

Adjust $\mathrm{pH}$ to 7.4 with $\mathrm{NaOH}$ or $\mathrm{HCl}$. Filter through $0.2 \mu \mathrm{m}$ membrane. Store at room temperature.

\subsubsection{Lysis buffer B}

\begin{tabular}{|lll|}
\hline Item & Concentration & Supplier \\
\hline $\mathrm{NaCl}$ & $500 \mathrm{mM}$ & Thermo Fisher Scientific New Zealand Ltd. \\
$\mathrm{NaH}_{2} \mathrm{PO}_{4}$ & $20 \mathrm{mM}$ & Thermo Fisher Scientific New Zealand Ltd. \\
Imidazole & $10 \mathrm{mM}$ & JT Baker Chemical Co. \\
Guanidine $\mathrm{HCl}$ & $6 \mathrm{M}$ & Life Technologies Inc. \\
Glycerol & $10 \%(\mathrm{v} / \mathrm{v})$ & Thermo Fisher Scientific New Zealand Ltd. \\
Dithiothreitol & $1 \mathrm{mM}$ & Roche Diagnostics N.Z., Ltd. \\
\hline
\end{tabular}

Adjust $\mathrm{pH}$ to 7.4 with $\mathrm{NaOH}$ or $\mathrm{HCl}$. Filter through $0.2 \mu \mathrm{m}$ membrane. Store at $4{ }^{\circ} \mathrm{C}$.

\subsubsection{Wash buffer B}

\begin{tabular}{|lll|}
\hline Item & Concentration & Supplier \\
\hline $\mathrm{NaCl}$ & $500 \mathrm{mM}$ & Thermo Fisher Scientific New Zealand Ltd. \\
$\mathrm{NaH}_{2} \mathrm{PO}_{4}$ & $20 \mathrm{mM}$ & Thermo Fisher Scientific New Zealand Ltd. \\
Imidazole & $20 \mathrm{mM}$ & JT Baker Chemical Co. \\
Guanidine $\mathrm{HCl}$ & $6 \mathrm{M}$ & Life Technologies Inc. \\
Glycerol & $10 \%(\mathrm{v} / \mathrm{v})$ & Thermo Fisher Scientific New Zealand Ltd. \\
Cysteine & $1 \mathrm{mM}$ & Sigma-Aldrich \\
Cystine & $0.1 \mathrm{mM}$ & Sigma-Aldrich \\
\hline
\end{tabular}

Adjust $\mathrm{pH}$ to 7.4 with $\mathrm{NaOH}$ or $\mathrm{HCl}$. Filter through $0.2 \mu \mathrm{m}$ membrane. Store at $4{ }^{\circ} \mathrm{C}$. 


\subsubsection{Refold buffer B}

\begin{tabular}{|lll|}
\hline Item & Concentration & Supplier \\
\hline $\mathrm{NaCl}$ & $500 \mathrm{mM}$ & Thermo Fisher Scientific New Zealand Ltd. \\
$\mathrm{NaH}_{2} \mathrm{PO}_{4}$ & $20 \mathrm{mM}$ & Thermo Fisher Scientific New Zealand Ltd. \\
Imidazole & $20 \mathrm{mM}$ & JT Baker Chemical Co. \\
Glycerol & $10 \%(\mathrm{v} / \mathrm{v})$ & Thermo Fisher Scientific New Zealand Ltd. \\
Cysteine & $1 \mathrm{mM}$ & Sigma-Aldrich \\
Cystine & $0.1 \mathrm{mM}$ & Sigma-Aldrich \\
\hline
\end{tabular}

Adjust $\mathrm{pH}$ to 7.4 with $\mathrm{NaOH}$ or $\mathrm{HCl}$. Filter through $0.2 \mu \mathrm{m}$ membrane. Store at $4{ }^{\circ} \mathrm{C}$.

\subsubsection{Elution buffer B}

\begin{tabular}{|lll|}
\hline Item & Concentration & Supplier \\
\hline $\mathrm{NaCl}$ & $500 \mathrm{mM}$ & Thermo Fisher Scientific New Zealand Ltd. \\
$\mathrm{NaH}_{2} \mathrm{PO}_{4}$ & $20 \mathrm{mM}$ & Thermo Fisher Scientific New Zealand Ltd. \\
Imidazole & $500 \mathrm{mM}$ & JT Baker Chemical Co. \\
Glycerol & $10 \%(\mathrm{v} / \mathrm{v})$ & Thermo Fisher Scientific New Zealand Ltd. \\
\hline
\end{tabular}

Adjust pH to 7.4 with $\mathrm{NaOH}$ or $\mathrm{HCl}$. Filter through $0.2 \mu \mathrm{m}$ membrane. Store at room temperature.

\subsubsection{Dialysis buffer}

\begin{tabular}{|lll|}
\hline Item & Concentration & Supplier \\
\hline $\mathrm{NaCl}$ & $150 \mathrm{mM}$ & Thermo Fisher Scientific New Zealand Ltd. \\
$\mathrm{NaH}_{2} \mathrm{PO}_{4}$ & $20 \mathrm{mM}$ & Thermo Fisher Scientific New Zealand Ltd. \\
\hline
\end{tabular}

Adjust $\mathrm{pH}$ to 7.4 with $\mathrm{NaOH}$ or $\mathrm{HCl}$. Filter through $0.2 \mu \mathrm{m}$ membrane. Store at room temperature.

\subsubsection{0× rEK cleavage capture buffer}

\begin{tabular}{|lll|}
\hline Item & Concentration & Supplier \\
\hline $\mathrm{NaCl}$ & $500 \mathrm{mM}$ & Thermo Fisher Scientific New Zealand Ltd. \\
Tris & $200 \mathrm{mM}$ & Fisher BioReagents \\
$\mathrm{CaCl}_{2}$ & $20 \mathrm{mM}$ & BDH Chemicals, Poole, England \\
\hline
\end{tabular}

Adjust $\mathrm{pH}$ to 7.4 with $\mathrm{HCl}$. Filter through $0.2 \mu \mathrm{m}$ membrane. Store at $4{ }^{\circ} \mathrm{C}$. 


\subsection{Solutions for protein detection}

\subsubsection{SDS-PAGE solutions}

\subsubsection{3× SLB}

\begin{tabular}{|lll|}
\hline Item & Concentration & Supplier \\
\hline Glycerol & $10 \%(\mathrm{v} / \mathrm{v})$ & Thermo Fisher Scientific New Zealand Ltd. \\
$\beta$-mercaptoethanol & $5 \%(\mathrm{v} / \mathrm{v})$ & Acros Organics, NJ, USA \\
SDS & $2 \%(\mathrm{w} / \mathrm{v})$ & Bio-Rad Laboratories Inc., CA, USA \\
Tris pH 6.8 & As required & Fisher BioReagents \\
Bromophenol blue & 1 or 2 grains & BDH Chemicals \\
\hline
\end{tabular}

Adjust $\mathrm{pH}$ to 6.8 with $\mathrm{HCl}$. Store at $-20{ }^{\circ} \mathrm{C}$.

\subsubsection{2 $4 \times$ stacking buffer}

\begin{tabular}{|lll|}
\hline Item & Concentration & Supplier \\
\hline Tris & $500 \mathrm{mM}$ & Fisher BioReagents \\
SDS & $0.4 \%(\mathrm{w} / \mathrm{v})$ & Bio-Rad Laboratories Inc. \\
\hline
\end{tabular}

Adjust pH to 6.9 with $\mathrm{HCl}$. Store at $4{ }^{\circ} \mathrm{C}$.

\subsubsection{3 $4 \times$ resolving buffer}

\begin{tabular}{|lll|}
\hline Item & Concentration & Supplier \\
\hline Tris & $1.5 \mathrm{M}$ & Fisher BioReagents \\
SDS & $0.4 \%(\mathrm{w} / \mathrm{v})$ & Bio-Rad Laboratories Inc. \\
\hline
\end{tabular}

Adjust $\mathrm{pH}$ to 8.8 with $\mathrm{HCl}$. Store at $4{ }^{\circ} \mathrm{C}$.

\subsubsection{10× Running buffer}

\begin{tabular}{|lll|}
\hline Item & Concentration & Supplier \\
\hline Tris & $25 \mathrm{mM}$ & Fisher BioReagents \\
Glycine & $192 \mathrm{mM}$ & Bio-Rad Laboratories Inc. \\
SDS & $0.1 \%(\mathrm{w} / \mathrm{v})$ & Bio-Rad Laboratories Inc. \\
\hline
\end{tabular}

Store at $4{ }^{\circ} \mathrm{C}$. 


\subsubsection{Fixative/destain solution}

\begin{tabular}{|lll|}
\hline Item & Concentration & Supplier \\
\hline Methanol & $40 \%(\mathrm{v} / \mathrm{v})$ & Fisher Scientific \\
Acetic acid & $10 \%(\mathrm{v} / \mathrm{v})$ & BDH Chemicals \\
\hline
\end{tabular}

Store at room temperature.

\subsubsection{CBB R-250 stain solution}

\begin{tabular}{|lll|}
\hline Item & Concentration & Supplier \\
\hline CBB R-250 & $0.1 \%(\mathrm{w} / \mathrm{v})$ & Bio-Rad Laboratories Inc., CA, USA \\
Methanol & $20 \%(\mathrm{v} / \mathrm{v})$ & Fisher Scientific \\
Acetic acid & $10 \%(\mathrm{v} / \mathrm{v})$ & BDH Chemicals \\
\hline
\end{tabular}

Store at room temperature.

\subsubsection{Western blot, dot blot and far-western blot solutions}

\subsubsection{CAPS transfer buffer}

\begin{tabular}{|lll|}
\hline Item & Concentration & Supplier \\
\hline CAPS & $10 \mathrm{mM}$ & Sigma-Aldrich \\
Methanol & $10 \%(\mathrm{v} / \mathrm{v})$ & Fisher Scientific, Loughborough, UK \\
\hline
\end{tabular}

Store at room temperature.

\subsubsection{Ponceau S stain solution}

\begin{tabular}{|lll|}
\hline Item & Concentration & Supplier \\
\hline Ponceau S & $0.1 \%(\mathrm{w} / \mathrm{v})$ & Sigma-Aldrich \\
Acetic acid & $5 \%(\mathrm{v} / \mathrm{v})$ & BDH Chemicals \\
\hline
\end{tabular}

Store at room temperature.

\subsubsection{Blocking solution A}

\begin{tabular}{|lll|}
\hline Item & Concentration & Supplier \\
\hline Non-fat skim milk & $4 \%(\mathrm{w} / \mathrm{v})$ & Foodstuffs Ltd., Auckland, New Zealand \\
PBST & To volume required & See 2.3.2.5 \\
\hline
\end{tabular}

Make fresh as required. Discard after use. 


\subsubsection{Blocking solution B}

\begin{tabular}{|lll|}
\hline Item & Concentration & Supplier \\
\hline Non-fat skim milk & $4 \%(w / v)$ & Foodstuffs Ltd., Auckland, New Zealand \\
TBST & To volume required & See 2.3.2.6 \\
\hline
\end{tabular}

Make fresh as required. Discard after use.

\subsubsection{Wash solution A}

\begin{tabular}{|lll|}
\hline Item & Concentration & Supplier \\
\hline PBS & $1 \mathrm{~L}$ & See 1.1 \\
Tween 20 & $0.05 \%(\mathrm{v} / \mathrm{v})$ & BDH Chemicals \\
\hline
\end{tabular}

Store at room temperature.

\subsubsection{Wash solution B}

\begin{tabular}{|lll|}
\hline Item & Concentration & Supplier \\
\hline TBS & $1 \mathrm{~L}$ & See 1.2 \\
Tween 20 & $0.05 \%(\mathrm{v} / \mathrm{v})$ & BDH Chemicals \\
\hline
\end{tabular}

Store at room temperature.

\subsubsection{Antibody diluent A}

\begin{tabular}{|lll|}
\hline Item & Concentration & Supplier \\
\hline BSA & $0.1 \%(w / v)$ & MP Biomedicals \\
PBST & To volume required & See 2.3.2.5 \\
\hline
\end{tabular}

Store at $4{ }^{\circ} \mathrm{C}$.

\subsubsection{Antibody diluent B}

\begin{tabular}{|lll|}
\hline Item & Concentration & Supplier \\
\hline BSA & $0.1 \%(w / v)$ & MP Biomedicals \\
TBST & To volume required & See 2.3.2.6 \\
\hline
\end{tabular}

Store at $4{ }^{\circ} \mathrm{C}$. 


\section{Solutions for neutrophil function assays}

\subsection{Serum-free RPMI 1640 medium}

\begin{tabular}{|c|c|c|}
\hline Item & Concentration & Supplier \\
\hline RPMI 1640 & $1 \times$ & Life Technologies Inc. \\
\hline $\begin{array}{l}\text { Penicillin \& } \\
\text { Streptomycin }\end{array}$ & $1 \mathrm{U} / \mathrm{ml} \& 1 \mu \mathrm{g} / \mathrm{ml}$ & Life Technologies Inc. \\
\hline GlutaMAX & $2 \mathrm{mM}$ & Life Technologies Inc. \\
\hline
\end{tabular}

Store at $4{ }^{\circ} \mathrm{C}$.

\subsection{0x Annexin-V binding buffer}

\begin{tabular}{|lll|}
\hline Item & Concentration & Supplier \\
\hline $\mathrm{HEPES}$ & $100 \mathrm{mM}$ & BDH Chemicals \\
$\mathrm{NaCl}$ & $1.4 \mathrm{M}$ & Thermo Fisher Scientific New Zealand Ltd. \\
$\mathrm{CaCl}_{2}$ & $25 \mathrm{mM}$ & BDH Chemicals \\
\hline
\end{tabular}

Adjust $\mathrm{pH}$ to 7.4 with $\mathrm{HCl}$. Filter through $0.2 \mu \mathrm{m}$ membrane. Store at $4{ }^{\circ} \mathrm{C}$. 


\section{Solutions for protein interaction assays}

\subsection{0× Protease inhibitor stock}

\begin{tabular}{|lll|}
\hline Item & Concentration & Supplier \\
\hline $\begin{array}{l}\text { Complete mini } \\
\text { EDTA-free protease } \\
\text { inhibitor tablets }\end{array}$ & 5 tablets & Roche Diagnostics N.Z., Ltd. \\
MQ. $\mathrm{H}_{2} \mathrm{O}$ & $5 \mathrm{ml}$ & \\
\hline
\end{tabular}

Store at $-20{ }^{\circ} \mathrm{C}$.

\subsection{Fractionation buffer}

\begin{tabular}{|lll|}
\hline Item & Concentration & Supplier \\
\hline HEPES pH 7.4 & $10 \mathrm{mM}$ & BDH Chemicals \\
$\mathrm{MgCl}_{2}$ & $2.5 \mathrm{mM}$ & Thermo Fisher Scientific New Zealand Ltd. \\
EGTA & $5 \mathrm{mM}$ & Sigma-Aldrich \\
Sucrose & $250 \mathrm{mM}$ & Sigma-Aldrich \\
PMSF & $0.1 \mathrm{mM}$ & Sigma-Aldrich \\
Protease inhibitors & $1 \times$ stock & See 4.1 \\
\hline
\end{tabular}

Make fresh as required. Add PMSF and protease inhibitors prior to use.

\subsection{Hypotonic buffer}

\begin{tabular}{|lll|}
\hline Item & Concentration & Supplier \\
\hline Tris- $\mathrm{HCl}, \mathrm{pH} 7.4$ & $20 \mathrm{mM}$ & Fisher BioReagents \\
$\mathrm{NaCl}$ & $10 \mathrm{mM}$ & Thermo Fisher Scientific New Zealand Ltd. \\
$\mathrm{MgCl} 2$ & $3 \mathrm{mM}$ & Thermo Fisher Scientific New Zealand Ltd. \\
$\mathrm{NaF}$ & $5 \mathrm{mM}$ & BDH Chemicals \\
$\mathrm{Na} 3 \mathrm{VO} 4$ & $1 \mathrm{mM}$ & Sigma-Aldrich \\
EDTA & $5 \mathrm{mM}$ & Thermo Fisher Scientific New Zealand Ltd. \\
PMSF & $1 \mathrm{mM}$ & Sigma-Aldrich \\
Protease inhibitors & $1 \times$ stock & See 4.1 \\
\hline
\end{tabular}

Make fresh as required. Add PMSF and protease inhibitors prior to use. 


\section{Mass spectrometry solutions}

\subsection{MS gel fixative solution}

\begin{tabular}{|lll|}
\hline Item & Concentration & Supplier \\
\hline Methanol & $50 \%(\mathrm{v} / \mathrm{v})$ & Fisher Scientific \\
Phosphoric acid & $2 \%(\mathrm{v} / \mathrm{v})$ & Sigma-Aldrich \\
\hline
\end{tabular}

Store at room temperature.

\subsection{CBB G-250 stain solution}

\begin{tabular}{|lll|}
\hline Item & Concentration & Supplier \\
\hline CBB G-250 & $0.25 \%(\mathrm{w} / \mathrm{v})$ & Bio-Rad Laboratories Inc. \\
Methanol & $40 \%(\mathrm{v} / \mathrm{v})$ & Fisher Scientific \\
Acetic acid & $10 \%(\mathrm{v} / \mathrm{v})$ & BDH Chemicals \\
\hline
\end{tabular}

Store at room temperature.

\subsection{MS gel destain solution}

\begin{tabular}{|lll|}
\hline Item & Concentration & Supplier \\
\hline Methanol & $5 \%(\mathrm{v} / \mathrm{v})$ & Fisher Scientific \\
\hline
\end{tabular}

Store at room temperature.

\subsection{Destain wash solution}

\begin{tabular}{|lll|}
\hline Item & Concentration & Supplier \\
\hline $\mathrm{NH}_{4} \mathrm{HCO}_{3}$ & $25 \mathrm{mM}$ & Sigma-Aldrich \\
Acetonitrile & $50 \%(\mathrm{v} / \mathrm{v})$ & Merck, Darmstadt, Germany \\
\hline
\end{tabular}

Make fresh as required.

\subsection{Trypsin digest solution}

\begin{tabular}{|lll|}
\hline Item & Concentration & Supplier \\
\hline Trypsin & $50 \mu \mathrm{g} / \mathrm{ml}$ & Promega, WI, USA \\
$\mathrm{NH}_{4} \mathrm{HCO}_{3}$ & $25 \mathrm{mM}$ & Sigma-Aldrich \\
$\mathrm{ObG}$ & $0.1 \%(\mathrm{w} / \mathrm{v})$ & Calbiochem, CA, USA \\
\hline
\end{tabular}

Make fresh as required. 


\subsection{TFA working solution (Trifluoroacetic acid)}

\begin{tabular}{|lll|}
\hline Item & Concentration & Supplier \\
\hline TFA & $2.5 \%(\mathrm{v} / \mathrm{v})$ & Merck \\
\hline
\end{tabular}

Make fresh as required.

\subsection{Acetonitrile/TFA Solution}

\begin{tabular}{|lll|}
\hline Item & Concentration & Supplier \\
\hline Acetonitrile & $50 \%(\mathrm{v} / \mathrm{v})$ & Merck \\
TFA & $0.1 \%(\mathrm{v} / \mathrm{v})$ & Merck \\
\hline
\end{tabular}

Make fresh as required.

\subsection{Matrix solution}

\begin{tabular}{|lll|}
\hline Item & Concentration & Supplier \\
\hline CHCA & $10 \mathrm{mg} / \mathrm{ml}$ & Bruker Daltonics $\mathrm{GmbH}$, Leipzig, Germany \\
Acetonitrile & $67 \%(\mathrm{v} / \mathrm{v})$ & Merck \\
TFA & $0.1 \%(\mathrm{v} / \mathrm{v})$ & Merck \\
\hline
\end{tabular}

Make fresh as required.

\subsection{Spot wash solution}

\begin{tabular}{|lll|}
\hline Item & Concentration & Supplier \\
\hline Ethanol & $80 \%(\mathrm{v} / \mathrm{v})$ & Thermo Fisher Scientific New Zealand Ltd. \\
TFA & $0.1 \%(\mathrm{v} / \mathrm{v})$ & Merck \\
\hline
\end{tabular}

Make fresh as required. 